\title{
Spanish Colonial Documents Pertaining to Mission Santa Cruz de San Saba (41MN23), Menard County, Texas
}

\author{
Mariah F. Wade \\ Jennifer K. McWilliams \\ Texas Historical Commission \\ Douglas K. Boyd
}

Follow this and additional works at: https://scholarworks.sfasu.edu/ita

Part of the American Material Culture Commons, Archaeological Anthropology Commons, Environmental Studies Commons, Other American Studies Commons, Other Arts and Humanities Commons, Other History of Art, Architecture, and Archaeology Commons, and the United States History Commons

Tell us how this article helped you.

This Article is brought to you for free and open access by the Center for Regional Heritage Research at SFA ScholarWorks. It has been accepted for inclusion in Index of Texas Archaeology: Open Access Gray Literature from the Lone Star State by an authorized editor of SFA ScholarWorks. For more information, please contact cdsscholarworks@sfasu.edu. 


\section{Spanish Colonial Documents Pertaining to Mission Santa Cruz de San Saba (41MN23), Menard County, Texas}

\section{Licensing Statement}

This is a work for hire produced for the Texas Department of Transportation (TxDOT), which owns all rights, title, and interest in and to all data and other information developed for this project under its contract with the report producer. The report may be cited and brief passages from this publication may be reproduced without permission provided that credit is given to TxDOT and the firm that produced it. Permission to reprint an entire chapter, section, figures or tables must be obtained in advance from the Supervisor of the Archeological Studies Branch, Environmental Affairs Division, Texas Department of Transportation, 125 East 11th Street, Austin, Texas, 78701. 


\title{
SPANISH COLONIAL DOCUMENTS PERTAINING TO MISSION SANTA CRUZ DE SAN SABÁ (4IMN23), MENARD COUNTY, TEXAS
}

\author{
Transcribed and Translated by \\ Mariah F. Wade \\ with a foreword by \\ Jennifer K. McWilliams \\ and \\ Douglas K. Boyd
}

Principal Investigator: Douglas K. Boyd

REPORTS OF INVESTIGATIONS, NUMBER 154

Prewitt and Associates, Inc.

Cultural Resources Services

Austin, Texas

ARCHELOGICAL STUDIES PROGRAM, REPORT NUMBER 102

Texas Department of Transportation

Environmental Affairs Division

Austin, Texas

CSJ No. 2008-01-091

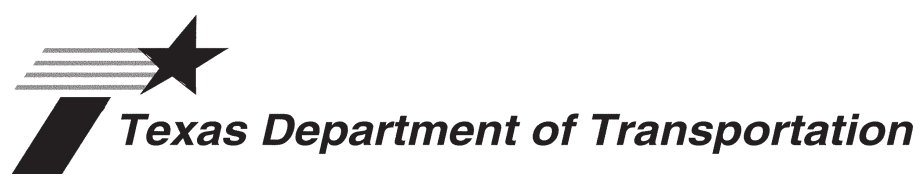

December 2007 


\title{
SPANISH COLONIAL DOCUMENTS PERTAINING TO MISSION SANTA CRUZ DE SAN SABÁ (4IMN23), MENARD COUNTY, TEXAS
}

\author{
COPYRIGHT @ 2007
}

Texas Department of Transportation (TxDOT) and Prewitt and Associates, Inc. (PAI)

TxDOT and PAI jointly own all rights, title, and interest in and to all data and other information developed for this project under Contract 575XXSA006, Work Authorization No. 57540SA006.

Brief passages from this publication may be reproduced without permission provided that credit is given to TxDOT and PAI. Permission to reprint an entire chapter, section, figures or tables must be obtained in advance from the Supervisor of the Archeological Studies

Program, Environmental Affairs Division, Texas Department of Transportation, 125 East 11th Street, Austin, Texas, 78701.

jointly published by the

Texas Department of Transportation

Environmental Affairs Division

Archeological Studies Program

Scott Pletka, Ph.D., Supervisor, Archeological Studies Program

Archeological Studies Program, Report Number 102

Al McGraw, Series Editor

and

Prewitt and Associates, Inc.

Cultural Resources Services

Austin, Texas

PAI Project Nos. 206025 \& 207047

Reports of Investigations, Number 154

Printed by Morgan Printing in Austin, Texas

ISBN 1-930788-71-1 


\section{TABLE OF CONTENTS}

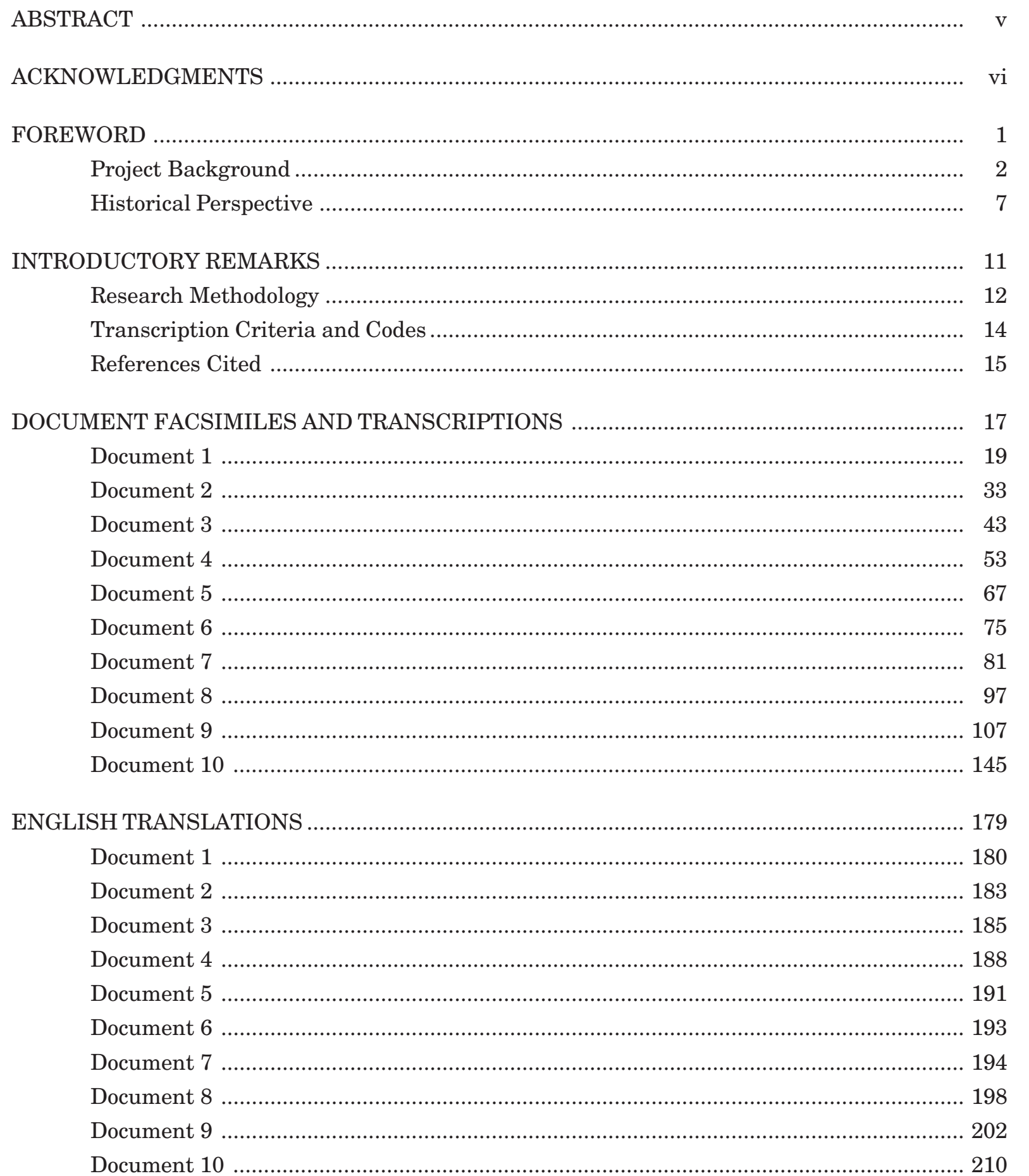





\begin{abstract}
The Texas Department of Transportation (TxDOT) is widening a 5-mile-long section of FM 2092 west of Menard in Menard County, Texas. The highway passes immediately south of the site of the Mission Santa Cruz de San Sabá (41MN23). Built in 1757 and destroyed in 1758, the mission is a time capsule of Spanish colonial archeology in the northern frontier of New Spain, along with the related Presidio de las Amarillas (41MN1, popularly known as Presidio San Sabá), which was occupied from 1757 to 1768 . The presidio location has long been known, but researchers did not rediscover the mission site until 1993. Texas Tech University conducted intensive archeological investigations at the mission site from 1993 through 1997. In the spring of 2006, the Texas Department of Transportation (TxDOT) contracted Prewitt and Associates, Inc., to conduct an archeological survey of this portion of FM 2092. The work included archeological testing of the portion of the mission site that extended into the road right of way. Intact and significant mission archeological remains were found, and data recovery investigations were subsequently undertaken in 2006-2007.

In conjunction with the FM 2092 survey effort, TxDOT sought to locate, transcribe, and translate previously unpublished or unknown Spanish colonial documents that might add to the story of the mission and presidio and the interpretation of the archeological remains of Mission San Sabá. Many Spanish documents pertaining to the mission and presidio have been translated and made available to researchers since the mid-twentieth century, but it is certain that additional documents exist and may offer further information on the lifeways and politics of those who occupied the mission. For this study, 10 previously unpublished Spanish documents—-those considered to be most relevant to Mission San Sabá-were selected and then transcribed and translated in their entirety. Each of these documents is presented here in three forms: a facsimile of the original handwritten Spanish text, a complete Spanish transcription of the document, and a complete English translation of the document.
\end{abstract}




\section{ACKNOWLEDGMENTS}

Thanks to all of the people who helped in the editing and production of this report. The Spanish transcriptions were read and edited by T. J. Campbell, and various drafts were edited by Doug Boyd, Jenny McWilliams, and Elaine Robbins from Prewitt and Associates and by John Arnn from the Archeological Studies Program, Environmental Affairs Division, Texas Department of Transportation. Sandra Hannum at Prewitt and Associates created the design and layout. Special thanks to TxDOT and Dr. Arnn for initiating this study and understanding the importance of archival documents for archeological research. 


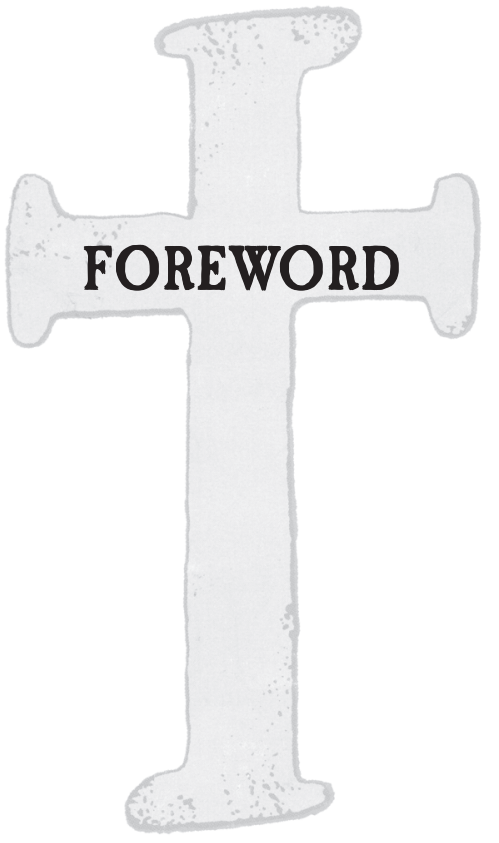




\section{PROJECT BACKGROUND}

In the spring of 2006, the Department of Transportation's Environmental Affairs Division (TxDOTENV) contracted Prewitt and Associates, Inc. (PAI), to conduct an archeological survey along a 4.9mile-long segment of FM 2092, extending from the eastern city limits of Menard eastward down the San Saba River valley to just past Fivemile Crossing (the first point where FM 2092 crosses the San Saba River). This segment of highway passes through a historically significant portion of Menard County and is immediately adjacent to the site of Mission Santa Cruz de San Sabá (41MN23). As with most Spanish colonial missionary efforts, the mission was accompanied by the establishment of a protective fort, Presidio de las Amarillas ${ }^{1}$ (41MN1), the archeological remains of which are 3.9 miles west of the mission on the west side of Menard. It is believed that this section of FM 2092 follows approximately the same route that the Spanish traveled between the presidio and the mission.

TxDOT proposes to widen FM 2092, adding shoulders to either side of the existing roadway. The plan is to increase the overall road width by $8 \mathrm{ft}$, but no new right of way will be required. In compliance with federal and state cultural resource laws, specifically Section 106 of the National Historic Preservation Act (16 USC 470 et seq.; 36 CFR 800) and the Antiquities Code of Texas (Texas Natural Resources Code, Title 9, Chapter 191; 13 TAC 26), TxDOT must take into account the potential impacts of the road construction on the cultural resources. The well-documented historical significance of this particular project corridor meant that the project would be subject to historical scrutiny and a detailed archeological inspection. Therefore, in addition to the typical archeological survey requirements generally expected in this situation, TxDOT expanded the scope of work to include intensive historical and archival research pertaining to the Spanish colonial history of the region along with an intensive geoarcheological investigation of the San Saba River valley in the project area and intensive subsurface survey in the vicinity of the mission. The archeological investigations

for the FM 2092 project were authorized by the Texas Historical Commission under authority of Texas Antiquities Permit No. 4192, and results of this work are reported by McWilliams et al. (2007).

PAI contracted Dr. Mariah Wade to conduct the archival research, including reviewing, tran- 
scribing, and translating Spanish documents. Prior to Dr. Wade's translations, most researchers had access only to secondary sources based on English-translated accounts published in a few wellknown manuscripts pertaining to Mission San Sabá, including Dunn’s (1914) “The Apache Mission on the San Saba River," Castañeda's (1936-1958) Our Catholic Heritage in Texas, 1519-1936, Nathan and Simpson's (1959, 2000) The San Sabá Papers: A Documentary Account of the Founding and Destruction of San Sabá Mission, Weddle's (1964, 1999) The San Sabá Mission: Spanish Pivot in Texas, Gilmore's (1967) A Documentary and Archaeological Investigation of Presidio San Luis de las Amarillas and Mission Santa Cruz de San Saba, and Hindes et al.'s (1995) The Rediscovery of Santa Cruz de San Sabá, A Mission for the Apache in Spanish Texas. While each of these publications offers similar versions of this period in history, all are generally taken from previous publications that utilized only a handful of documents available to early authors. With the exception of Gilmore (1967) and a complete translated document by Dennis (1995) in Hindes et al. (1995), these works provide only limited excerpts of transcriptions (generally footnotes), leaving the reader no means of evaluating the accuracy of the translations. Current research reporting standards, such as the methodologies established by the Arizona State Museum (2006) and Barnes et al. (1981), dictate that the original document, a complete transcription, and a complete translation are all published. Wade followed these protocols for the archival research reported in this manuscript.

Wade reviewed over 150 Spanish documents from various central Texas archives-primarily in the Celaya Collection, Old Spanish Missions Collection at Our Lady of the Lake University in San Antonio, and the Center for American History at the University of Texas at Austin. The documents, which varied from 1 to 30 pages in length, are handwritten Spanish texts that had never been fully transcribed and translated. Wade then prepared an executive summary of all the documents she had reviewed and submitted it to PAI and TxDOT-ENV in June 2006. The executive summary highlighted details of archeological interest in the mission area, especially any remarks that contained information pertinent to possible Spanish or Native American archeological remains that might be encountered in or around the mission. This work was completed before PAI began field investigations in the mission area in July 2006.

After reviewing the documents to determine their contents, Wade chose 10 to be transcribed and translated in their entirety. She also prepared a brief historiography that describes what is known about the origin and historical context of each document that was translated. A summary of the historiography is provided in the following table. 
Spanish Colonial Documents Pertaining to Mission San Sabá

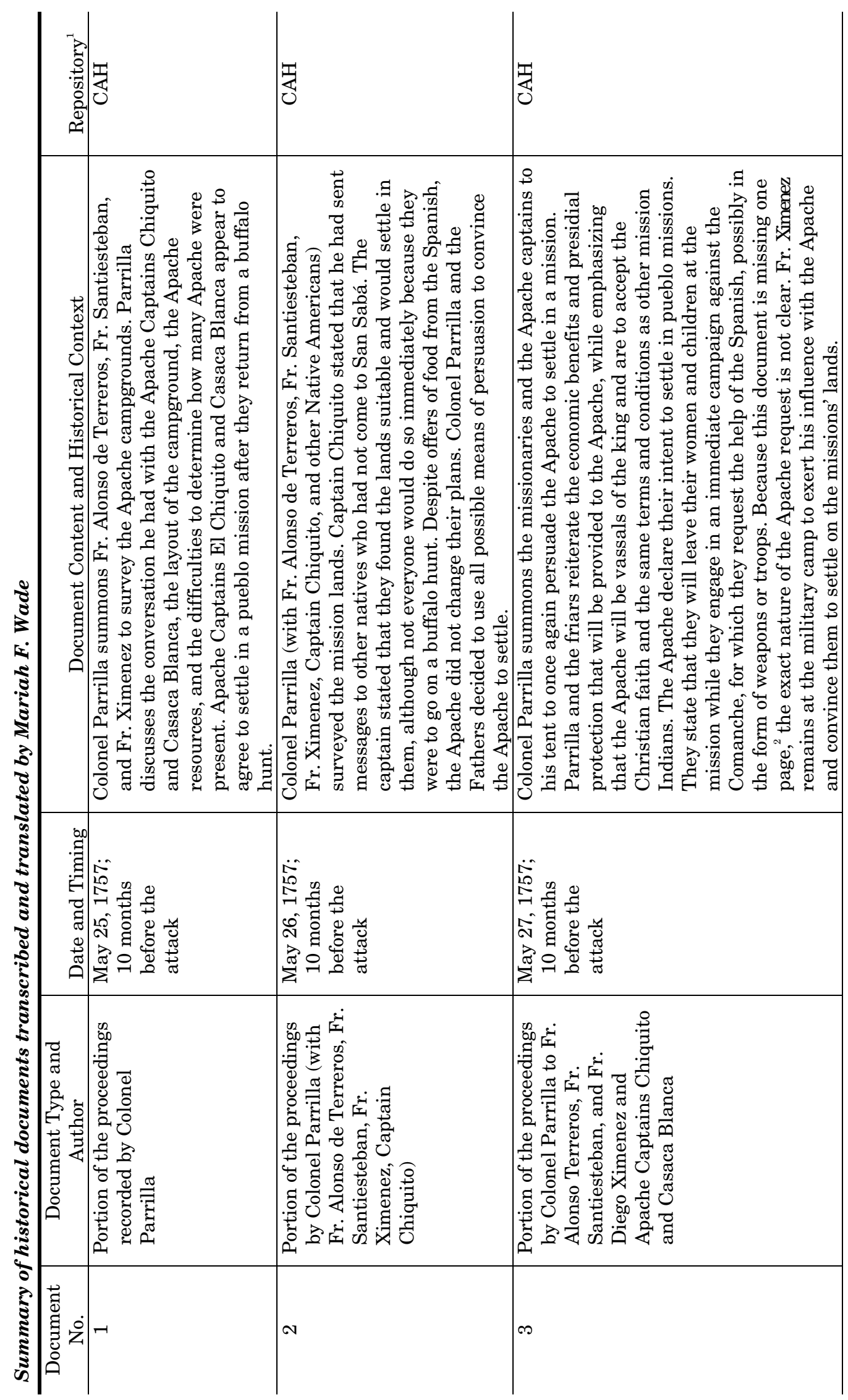




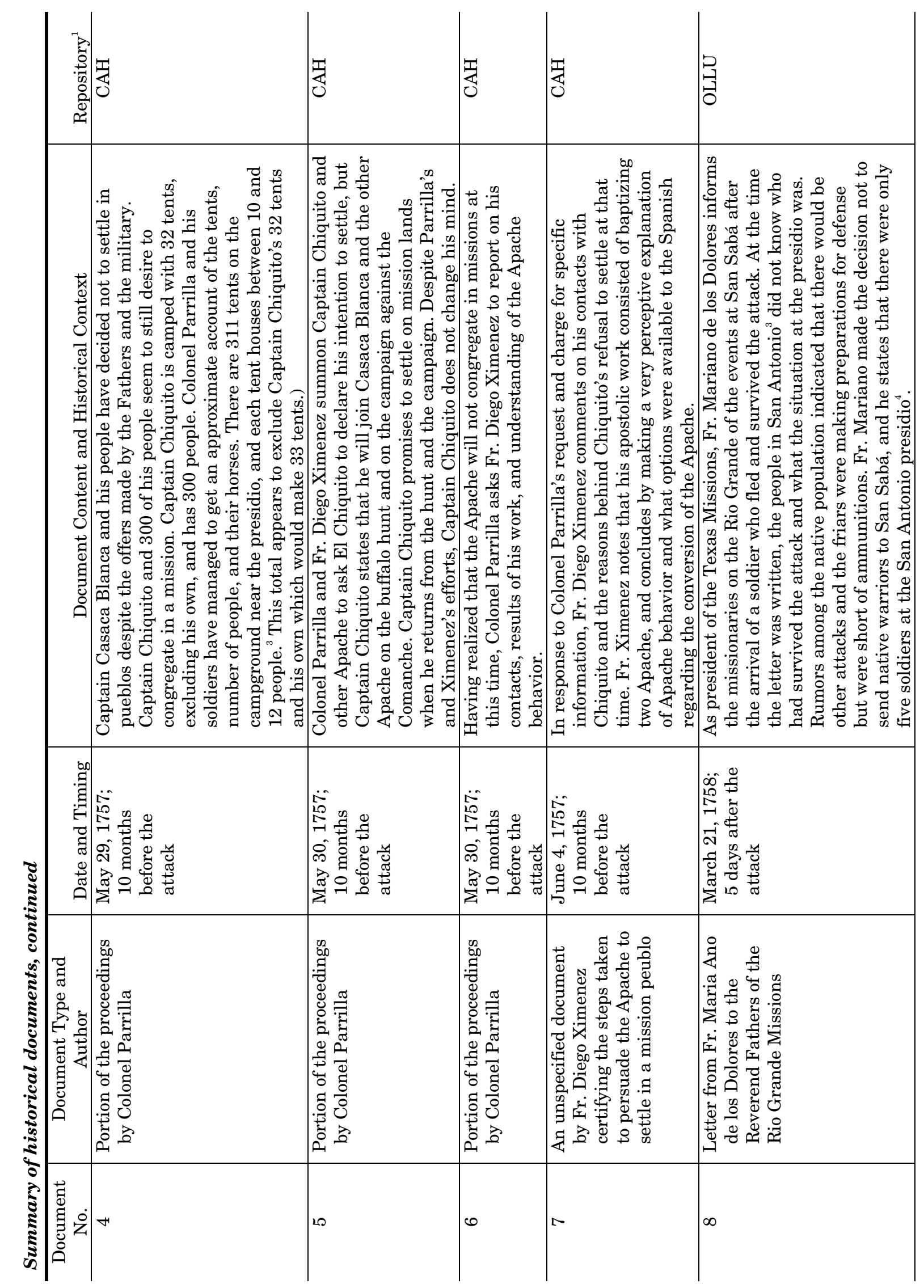


Spanish Colonial Documents Pertaining to Mission San Sabá

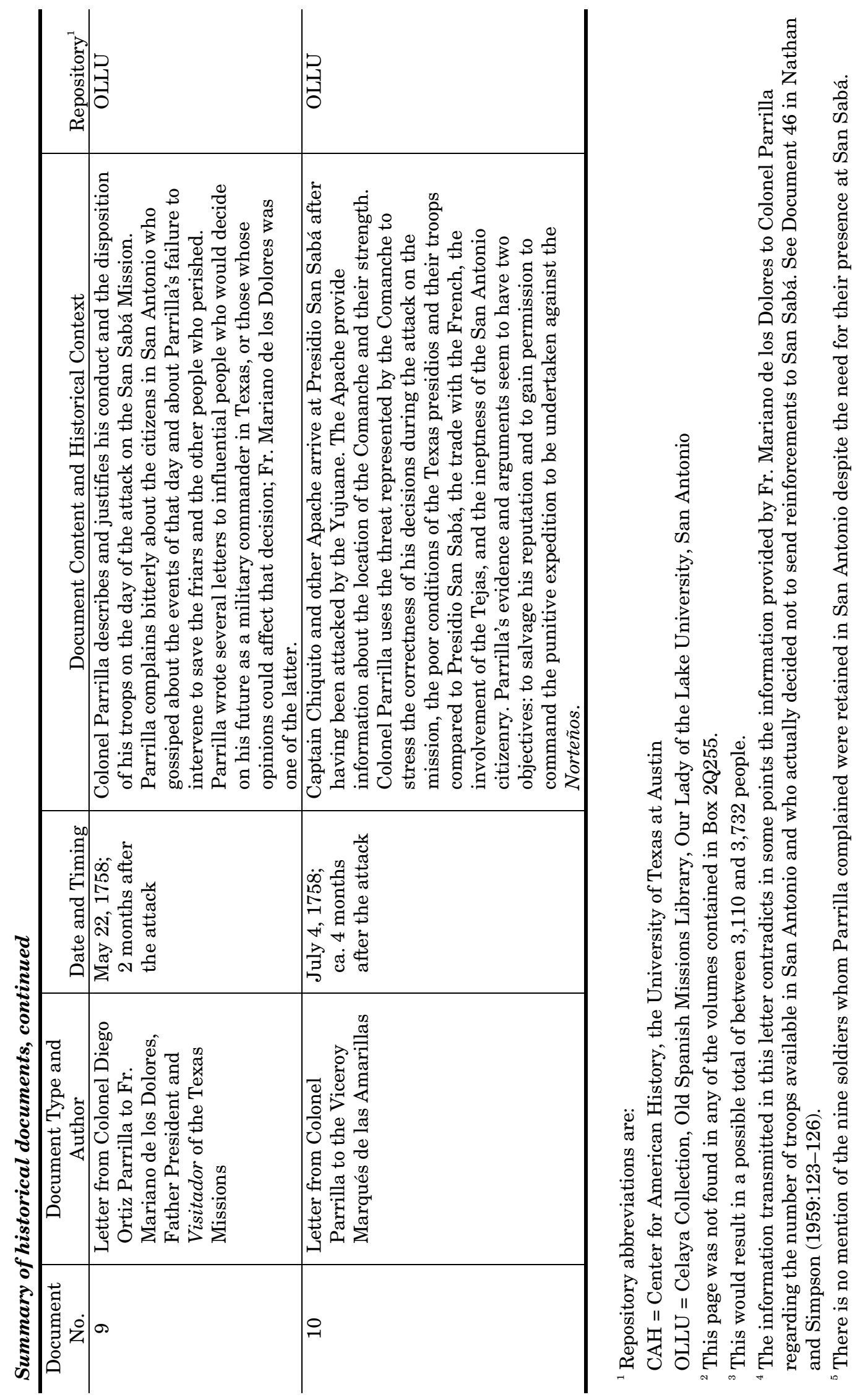




\section{HISTORICAL PERSPECTIVE}

The San Sabá Mission was established in 1757 by missionaries from the two primary colleges of New Spain: Colégio de Santa Cruz de Querétaro and San Fernando de México. A generous donation from a wealthy Spanish citizen, Don Pedro Romero de Terreros, facilitated the founding of the mission that was intended for the Lipan Apache. Leaving Mexico in late 1756, the soldiers and friars, along with native Tlaxcaltecan neophytes, moved northward to San Antonio to begin their trip northwestward to a location previously chosen on the San Saba River in the heart of Apache country. Undertones of devious politics and motives of personal gain run deeply through the history of the founding of the mission—and eventually led to its demise.

Arriving on the San Saba River in April 1757, the group constructed a simple, temporary wattleand-daub compound in what is now Menard County, Texas. With long-range plans to build at least two additional missions, the friars awaited the arrival of the Apaches to no avail. While some Apaches came to visit briefly, they had no intention of settling down there. The mission itself was short-lived, lasting only 11 months before its destruction by a mix of northern tribes commonly referred to as the Norteños. While 11 months is a brief time historically speaking, the documents from the mission period and the few months immediately following provide heart-wrenching personal details about the events that occurred and emotions felt as the hopeful undertaking turned to tragedy. There are no Native American documents that describe how the Norteños felt about the Spanish invaders and their alliance with their Apache enemies. In contrast, the Spanish view that is clearly demonstrated in the surviving documents is that of an unprovoked attack on the mission and the massacre of innocent Christians, with two murdered priests who became martyrs for the Catholic cause.

The 10 documents presented here span from May 25, 1757, to July 4, 1758, covering a period beginning 10 months prior to the destruction of the mission and ending 4 months after the attack. Colonel Parrilla, military commander of the associated protective presidio, wrote Documents 1-6 soon after the construction of the mission. These papers contain his thoughts on dealing with the friars, their attempts to attract the Lipan Apache into missionization, and the Spaniards' first realizations that the Indians might not congregate at the mission as they had hoped. Document 7, writ- 


\section{Spanish Colonial Documents Pertaining to Mission San Sabá}

ten by Fr. Ximenez soon after the previous documents, explains why the Indian chiefs chose not to settle at the mission. Document 8 was written by Fr. Dolores ${ }^{2}$, president of the San Antonio missions, five days after the attack. Fr. Dolores reportedly opposed the establishment of San Sabá Mission. In this document, he recounts the story of one of the soldiers who survived the attack, but it includes some misinformation because the survivor fled the attack and did not know the final outcome. Colonel Parrilla wrote Document 9, a letter to Fr. Dolores, the Father President of the San Antonio Missions, two months after the attack to provide justification for his conduct during the attack. Finally, Parrilla wrote Document 10 four months after the attack to the Viceroy Marqués de las Amarillas, for whom the presidio, known as San Luís de las Amarillas, was named. Again the letter is primarily a justification for his actions, but in it Parrilla also requests a punitive expedition against the Norteños.

These documents take us through a very important turning point in the Spanish history of the northern frontier. At the beginning, the Spanish proudly marched northward to conquer and Christianize the inhabitants of what is now Texas. The tone of the documents shifts from hopefulness to frustration as the reality of the mission effort sets in, followed by a sorrowful portrayal of a vengeful attack that abruptly ended the mission endeavor. The last documents demonstrate Parrilla's desire to tell his story, but they look to the future to avenge the violence of the Norteños.

These accounts do not provide precise locational information and unequivocal historical facts, as such accounts rarely do. They will never be a smoking gun for historical reinterpretation, but they offer many small bits of important information. The events and details described in individual documents are rather meaningless when viewed in isolation and out of context-much like an artifact removed from its place of deposition and without provenience. However, when the "facts" and "events" revealed from many different documents are interpreted together, the result is not necessarily the complete truth but a clearer understanding of what the contemporary writers believed to be the truth from their own cultural perspective.

The contribution of Mariah Wade's work is not that it provides final answers but that it adds significantly to the pieces of the puzzle. These documents are building blocks full of information for future researchers to use in combination with previously translated materials. By themselves, the 10 documents presented and examined herein will not change the way modern historians view the establishment and destruction of Mission San Sabá. It is hoped that the additional historical information they provide, which is presented in a concise format for critical interpretation, will serve two 
purposes. First, these documents may spark a renewed interest in archival study of the mission and presidio and their roles in the history of European and Native American interactions. Just as the recent reexamination of the Coronado documents has shed new light on the first Spanish entrada into the southwestern United States (Flint and Flint 1997, 2003, 2005), a critical reanalysis of all the San Sabá documents would be very beneficial ${ }^{3}$. And second, these documents may provide considerable aid in the interpretation of the physical remains that have been discovered during the recent archeological investigations of the San Sabá Mission and Presidio (see Hall 1994, 2001; Hindes et al. 1995; McWilliams 2001; McWilliams et al. 2007; Walter et al. 2003; Walter et al. 2005; Wolf 2005).

\footnotetext{
${ }^{1}$ Presidio San Luis de las Amarillas was the original name given to the presidio in 1756 and was used throughout Colonel Diego Ortiz de Parrilla's command. Parrilla was replaced by Captain Felipe de Rábago y Terán in 1760, and in 1762 the presidio was fortified by a stone construction. The presidio was renamed Real Presidio San Sabá at that time (Walter et al. 2003:3). Therefore, since this report concentrates on the mission, the presidio is referred by the name it was called when the mission existed, from 1757 to 1758 .

${ }^{2}$ Fr. Mariano de los Dolores and Fr. Maria Ano de los Dolores are both correct. Correspondence to him was usually addressed to Mariano, while he sometimes signed his name as Maria Ano (the year of Mary). It is likely that he was very devout to the Virgin Mary and proud of his name.

${ }^{3}$ Note that a recent book by Robert Weddle (2007), After the Massacre: The Violent Legacy of the San Sabá Mission, examines the historical significance of the massacre and the Spaniards' 1759 expedition to the Red River to punish the northern tribes that participated in the attack on the mission. The book presents translations of a relatively unknown diary pertaining to the expedition.
} 


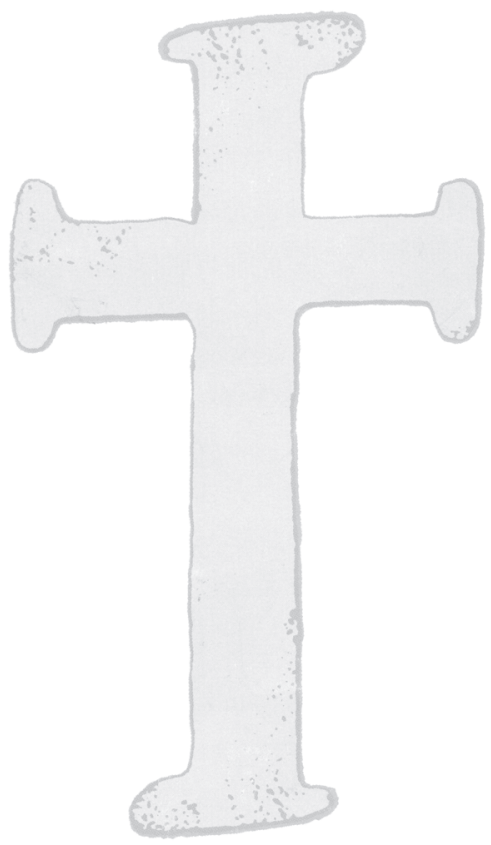




\section{INTRODUCTORY REMARKS}




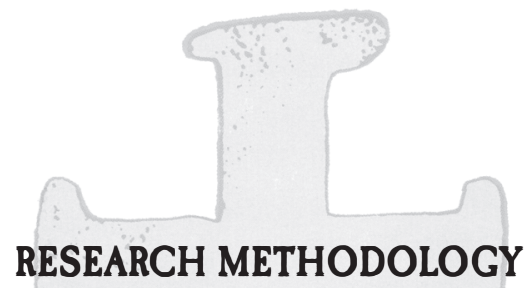

To fulfill the requirements of this contract, I reviewed and read over 150 documents of various lengths (from over 30 pages to 1 page); only a few were visually scanned. There is a great deal of duplication in the materials I have seen from the Center for American History and those in the Celaya Archives, Old Spanish Missions Collection, at Our Lady of the Lake University. The Center for American History has typescript versions done by various people of some of the documents in the Celaya Archives. Discrepancies between these and other documents likely exist, but they require a word-by-word reading, which is very time-consuming.

Apart from Roll 9 in the Celaya Archives, I reviewed the documents contained in the various volumes in the following archival boxes at the Center for American History: 2Q150; 2Q237; 2Q250; 2Q251; 2Q253; 2Q255; 2Q256, and 2Q258. There is indeed a wealth of material pertaining to the establishment of San Sabá Mission before and after the attack of San Sabá, but very little that specifically addressed the requirements of this project.

Documents 1 through 7 are translations and transcriptions of a series of documents that detail the proceedings taken by the army and the friars immediately after Colonel Diego Ortiz Parrilla inspected the San Saba River in April 1757; the survey documents were translated and published by Kathleen Gilmore in 1967. From these proceedings, I chose documents that included important cultural material about the Apache, about the location of campgrounds before the establishment of the mission, and about the background problems that plagued the establishment of the mission. One document (Document 6) was included to provide context and continuity. I strongly recommend that, if possible, the remainder of the documents included in the proceedings be translated.

Mark Wolf, who has conducted extensive scholarly research on San Sabá, provided Prewitt and Associates with three documents, two of which are translated and transcribed here (Documents 8 and 9). Both documents were penned after the attack of San Sabá-the first immediately after and the second two months after the attack. The third document provided by Mark Wolf is a copy of Fr. Miguel de Molina's declaration after the attack of San Sabá, which was translated and published by 
Paul Nathan and Lesley Simpson in 1959. A review of this copy did not show any significant documentary discrepancies to justify another translation and a lengthy transcription. To establish a thematic and temporal sequence with Wolf's documents and because of the emphasis given to the cultural material on the Apache as well as some unique information provided by the Apache on the Comanche, I translated and transcribed Document 10 from the Celaya Archives. This document, penned in July 1758, ties in with Colonel Parrilla's continuous efforts to clear his name, as seen in Document 9 , and his forceful appeal to be given the command of the expedition to punish the Norteños.

Regarding the translations, a few words were not translated, since they do not have clear counterparts in the English language, but their meaning in the text is rendered obvious by the context. Although every effort was made to remain faithful to the text, readers should not expect a word-forword correspondence between the Spanish and English texts. A question mark between brackets [?] and inserted immediately following a word or expression indicates that I am unsure of the meaning of the word or expression. To provide a readable document in the English language, sentence order and structure were frequently altered, particularly when sentences were too long and separated by colons or semicolons. In a few cases, the construction of the sentence and its meaning were obscure. In such cases, I interpreted the sentence to the best of my ability, often taking into account information acquired from other documents. Still, other interpretations are possible. 


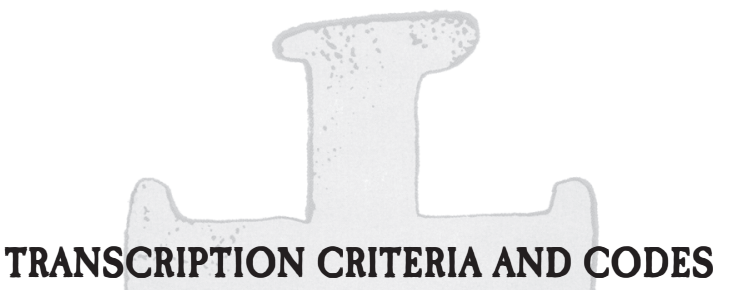

The original spelling, capitalization, punctuation, diacritical marks, and word divisions used in the documents have been carefully maintained. Diacritical marks (accents) were maintained whenever it was obvious that the scribe intended to use them. Diacritical marks over the $i$ were problematic because often the scribe did not differentiate a dotted $i$ from an accented $i$, as is the case for Document 9. In such cases, no accent was used.

Each individual folio is marked by a folio number in brackets either recto $(r)$ or verso (v); for instance [fol. 1r]. All documents follow the same arbitrary sequence. All translations show the break from one folio to another by the use of double slashes //.

Below is a key to all other transcription codes used:

- Folio lines are noted on the right side of the transcription text to facilitate reading, and the counting restarts with each new folio

- Paragraphs are indicated by II

- Addresses, titles, and marginal notes, other than continuous and intercalated text produced by the scribe, are preceded and indicated by flourished brackets \{\}

- Deletions, erasures, intercalations, and other corrections made by the scribe are shown between parenthesis with a caret preceding the correction (“^”)

- Changes or restorations made by the transcriber are shown between brackets []

- Illegible text is shown as [???]

- Restoration of abbreviations is shown in italics

- If the letter $n$ appears with a diacritic mark, it is shown as "ñ"

- Roman numerals are indicated by an arabic numeral followed by a , Transcription and editorial criteria have been adapted from Mackenzie (1981). 


\section{REFERENCES CITED}

Arizona State Museum

2006 Documentary Relations of the Southwest. Online database index to 1,500 microfilm reels of documents. Office of Ethnohistorical Research, Arizona State Museum. DRSW Master Index Online. Available from http://www.statemuseum.arizona.edu/oer/drswtool.shtml [accessed April 11, 2006].

Barnes, Thomas, C., Thomas H. Naylor, and Charles W. Polzer

1981 Northern New Spain: A Research Guide. University of Arizona Press, Tucson.

Castañeda, Carlos E.

1936-1958 Our Catholic Heritage in Texas, 1519-1936, 7 Volumes. Von Boeckmann-Jones Company, Austin.

Dennis, Philip A. (translator)

1995 Revenge of the Rio San Saba. In The Rediscovery of Santa Cruz de San Sabá, A Mission for the Apache in Spanish Texas, by Kay V. Hindes, Mark R. Wolf, Grant D. Hall, and Kathleen Kirk Gilmore, pp. 79-84. San Sabá Regional Survey Report 1. Archaeology Laboratory, Texas Tech University, Lubbock, and the Texas State Historical Association, Austin.

Dunn, William Edward

1914 The Apache Mission on the San Saba River: Its Founding and Failure. Southwestern Historical Quarterly 17(4):379-414.

Flint, Richard, and Shirley Cushing Flint (editors)

1997 The Coronado Expedition to Tierra Nueva: The 1540-1542 Route Across the Southwest. University Press of Colorado, Niwot.

2003 The Coronado Expedition: From the Distance of 460 Years. University of New Mexico Press, Albuquerque.

2005 Documents of the Coronado Expedition, 1539-1542: "They Were Not Familiar with His Majesty nor Did They Wish to Be His Subjects.” Edited, translated, and annotated by Richard Flint and Shirley Cushing Flint. Southern Methodist University Press, Dallas.

Gilmore, Kathleen

1967 A Documentary and Archaeological Investigation of Presidio San Luis de las Amarillas and Mission Santa Cruz de San Saba, Menard County, Texas. Archeological Program Report No. 9. State Building Commission, Austin.

Hall, Grant D.

1994 Searching for San Sabá. Heritage, Spring 1994: 12-15.

2001 Mission San Sabá. Texas Beyond History Web exhibit. Texas Archeological Research Laboratory, The University of Texas at Austin and the Texas Archeological Society. Available from http:// www.texasbeyondhistory.net/sansaba/index.html [accessed March 4, 2006].

Hindes, V. Kay, Mark R. Wolf, Grant D. Hall, and Kathleen Kirk Gilmore

1995 The Rediscovery of Santa Cruz de San Sabá, A Mission for the Apache in Spanish Texas. San Sabá Regional Survey Report 1, Archaeology Laboratory, Texas Tech University, Lubbock, and the Texas State Historical Association, Austin. 
Mackenzie, David

1981 A Manual of Manuscript Transcription for the Dictionary of the Old Spanish Language, Second Edition. The Hispanic Seminary of Medieval Studies Limited, The University of Wisconsin, Madison.

McWilliams, Jennifer K.

2001 Material Culture and Mission Inventories: A Comparison of Archaeological and Archival Data from the Mission Santa Cruz de San Sabá, Menard County, Texas. M.A. thesis, Texas Tech University, Lubbock.

McWilliams, Jennifer K., Douglas K. Boyd, and Céline Finney

2007 Historical Assessment and Geoarcheological Survey of 4.7 Miles of FM 2092 from Menard to Fivemile Crossing, Menard County, Texas. Archeological Studies Program Report No. 103. Environmental Affairs Division, Texas Department of Transportation, Austin. Reports of Investigations No. 155. Prewitt and Associates, Inc., Austin.

Nathan, Paul D., and Lesley Byrd Simpson (translator and editor)

1959 The San Saba Papers: A Documentary Account of the Founding and Destruction of San Saba Mission. John Howell-Books, San Francisco.

2000 The San Sabá Papers:A Documentary Account of the Founding and Destruction of San Sabá Mission. Southern Methodist University Press, Dallas.

Walter, Tamra L., Elizabeth A. Cooper, Nancy Zayatz, and Grant D. Hall

2003 The Ruin of Ruins: Archaeological Excavations at Presidio San Sabá, 41MN1, Menard County, Texas. 2000-2002 Field Expeditions. San Saba Regional Survey Report 2. Archaeology Laboratory, Texas Tech University, Lubbock.

Walter, Tamra L., Mark Wolf, and Bennett Kimball

2005 Spanish Colonial Presidio Architecture: An Example from Presidio San Sabá, Menard County, Texas. Bulletin of the Texas Archeological Society 76:29-44.

Weddle, Robert S.

1964 The San Sabá Mission: Spanish Pivot in Texas. University of Texas Press, Austin.

1999 The San Sabá Mission: Spanish Pivot in Texas. Texas A\&M University Press, College Station.

2007 After the Massacre: The Violent Legacy of the San Sabá Mission. Texas Tech University Press, Lubbock.

Wolf, Mark

2005 Presidio San Saba: A Case Study in Colonial Evolutionary Architecture. Bulletin of the Texas Archeological Society 76:45-59. 


\section{DOCUMENT FACSIMILES AND TRANSCRIPTIONS}




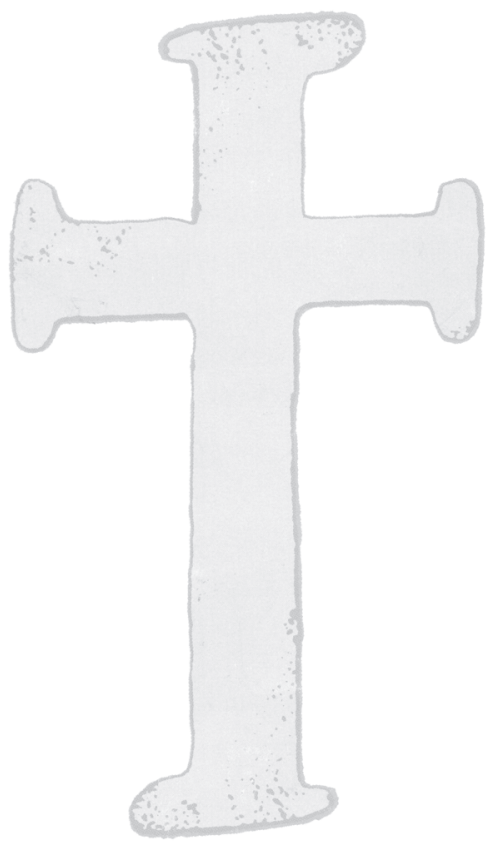




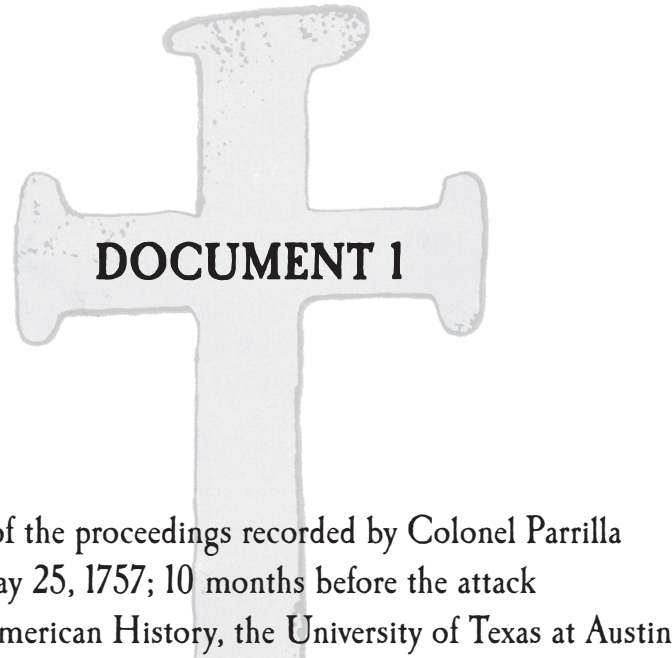


Spanish Colonial Documents Pertaining to Mission San Sabá

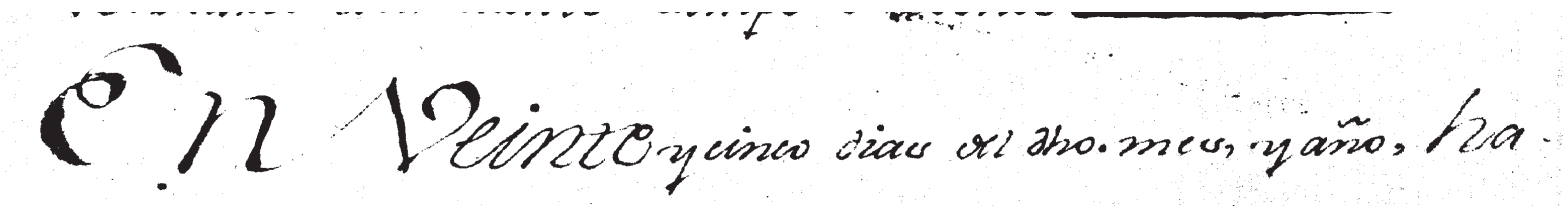

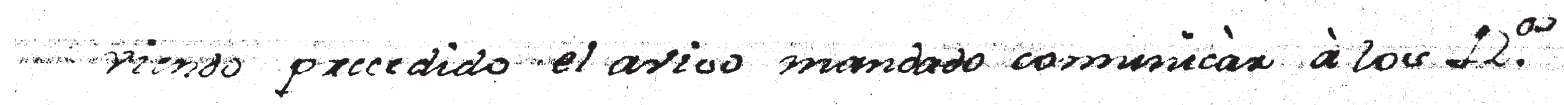

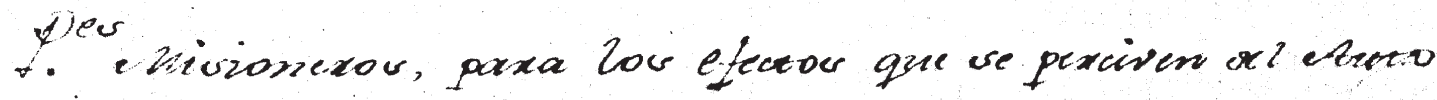

\%. precedes. Llegaxion à ene Campamont poco despues bes

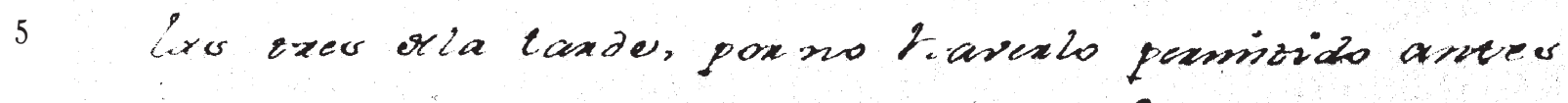

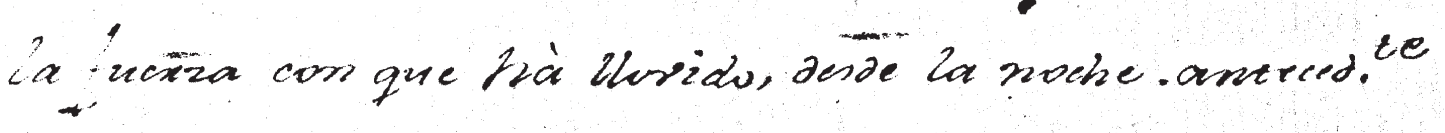

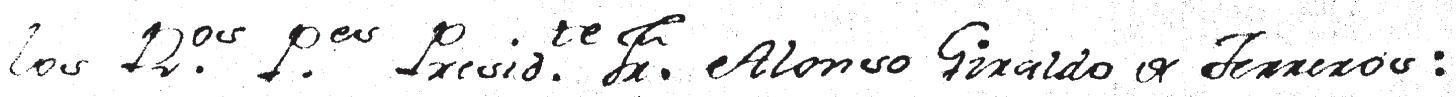

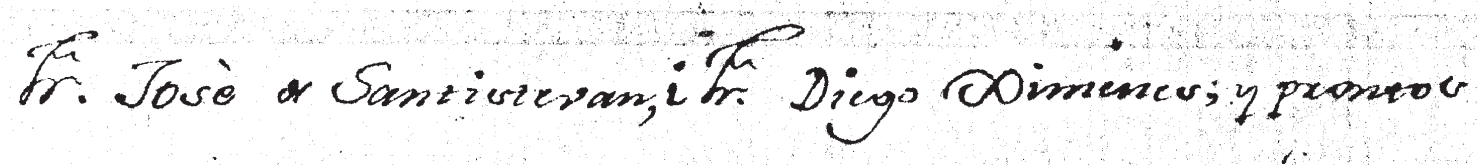


[fol. 1r]

\{top margin center\} \369

\{left margin\} 138

EN VEINTE y cinco dias del dicho mes, y año, ha-

viendo precedido el aviso mandado comunicàr à los Reverendos

Padres Misioneros, para los efectos que se perciven del Auto

que precede, llegaron à este Campamento, poco despues de

las tres dela tarde, por no haverlo permitido antes

la fuerza com que hà llovido, desde la noche antecedente

los Reverendos Padres Presidente Fray Alonso Giraldo de Terreros:

Fray Josè de Santistevan, i Fray Diego Ximines; y prontos 
Spanish Colonial Documents Pertaining to Mission San Sabá

$$
370
$$

in el Capition elor Apacher, recumocido pox el Claiquiar,

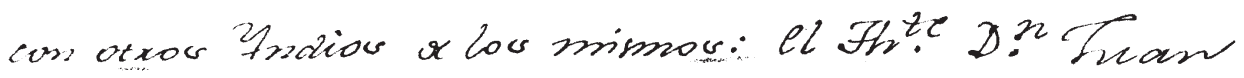

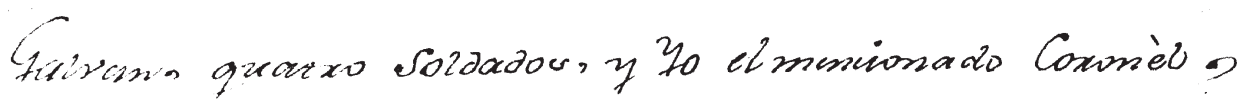

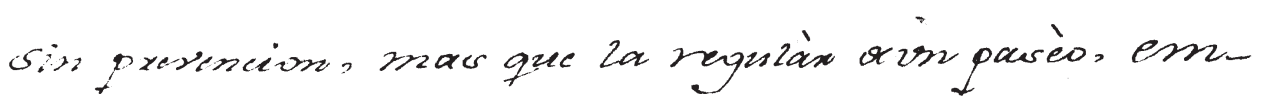
prehendimor à Carallo, pox enter el axcido nump ex Ficndar,

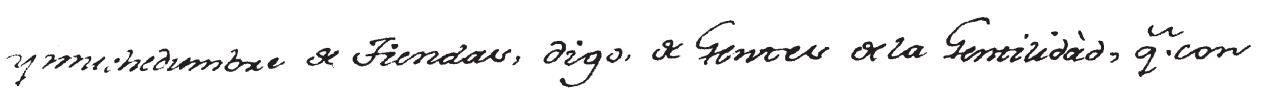
La inmediacion oha. à novotxos, se hallan acampudos' If haviondo abrextido en zos ismblanter etodov elgwro, if saticfiaccion que gozano la confiama qu les asisue en mu

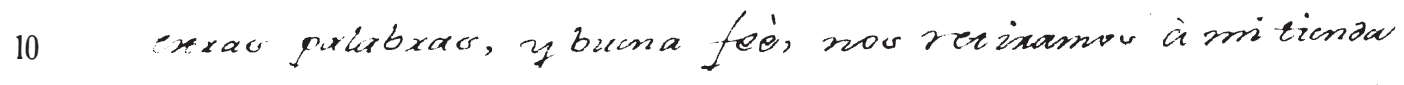

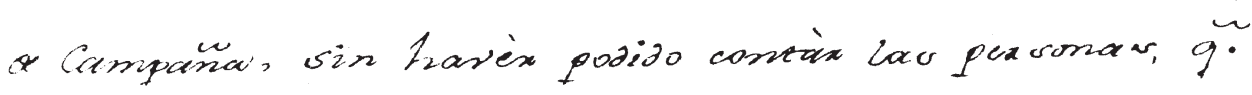

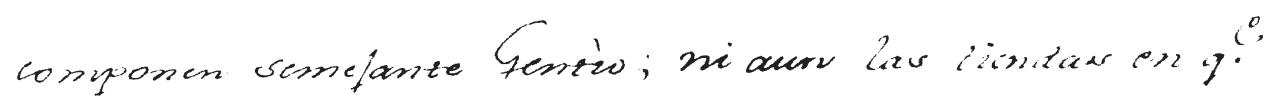

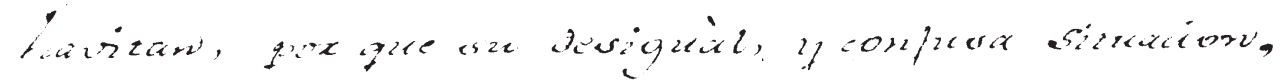

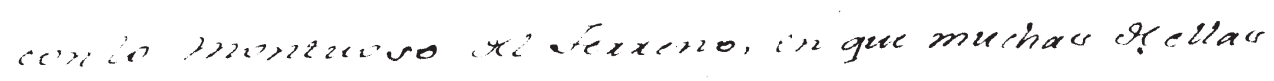

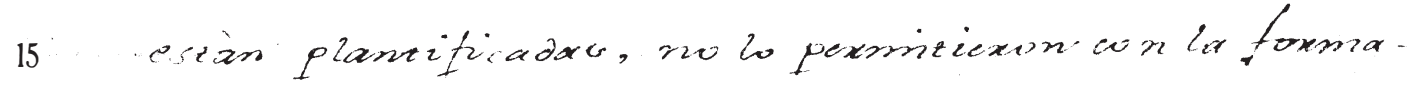

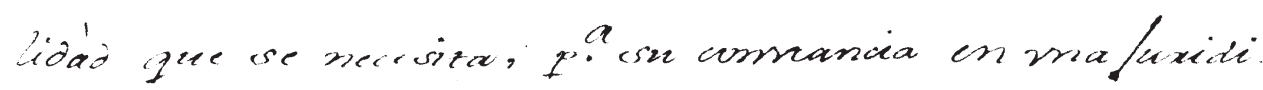

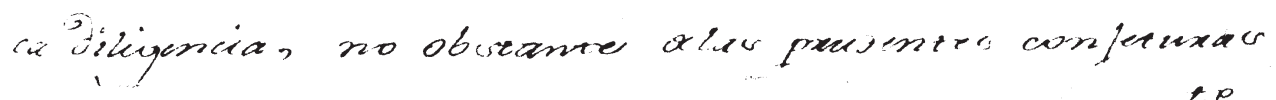

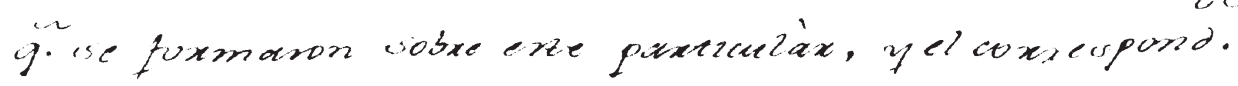
al cxecido numew of Ciscalor, y nutas, que zambien 20 turimov à la viseas pries aenque la cuenta ciexta, I rexidica, que debe conuexix, abxía sido facil consuguixla 
[fol. lv]

\{top margin center\} $\backslash 370$

\{left margin\} 139

yà el Capitàn de los Apaches, reconocido por el Chiquito, con otros Yndios de los mismos: el Theniente Don Juan Galvan, quatro soldados, y Yo el mencionado Coronèl, sin prevencion, mas que la regulàr de un pasèo, emprehendimos à Cavallo, por entre el crecido numero de Tiendas, y muchedumbre de Tiendas, digo, de Gentes de la Gentilidàd, que con la inmediacion dicha à nosotros, se hallan acampados; $\mathrm{y}$ haviendo advertido en los semblantes de todos elgusto, y satisfaccion que gozan, la confianza que les asiste en nuestras palabras, y buena feè, nos retiramos à mi tienda de Campaña, sin havèr podido contàr las personas, que componen semejante Gentìo; ni aun las tiendas en que havitan, por que su desiguàl, y confusa situacion, con lo montuoso del terreno, en que muchas de ellas estàn plantificadas, no lo permitieron con la forma-

lidàd que se necesita, para su constancia en una juridica diligencia, no obstante de las presentes conjecturas que se formaron sobre este particulàr, y el correspondente al crecido numero de Cavallos, y Mulas, que tambien tuvimos à la vista; pues aunque la cuenta cierta, y veridica, que debe concurrir, abría sido facil conseguirla 
pox qualquicia texmino; no se tuno pax canvon.

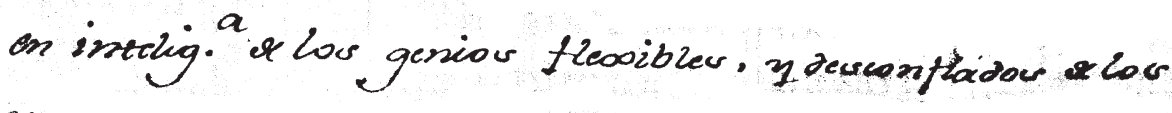
Fordios, If pux que se podxá eosecuitas enla ocavion de

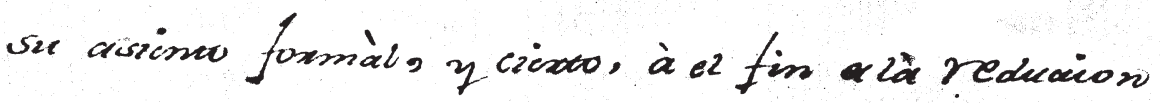

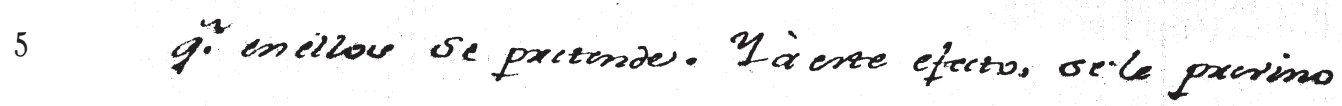
à los espoxesradoor Capizaner Chiquito, y cascacablanca, y à ozxor machor lindior aloo pxales, que conuaxiexon tambien enla dra. mi tiendas à quen-m pox el dia mañara se funten, I par en con migo,

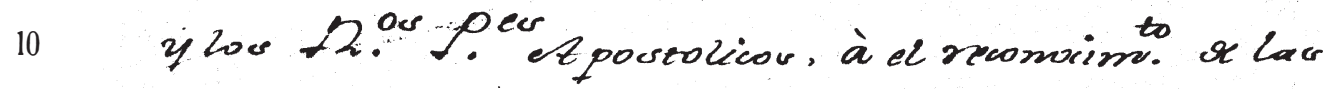

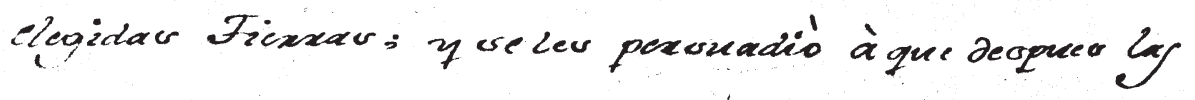
ocupasen en un pueble a Fiendatr, y formàl a vimto;

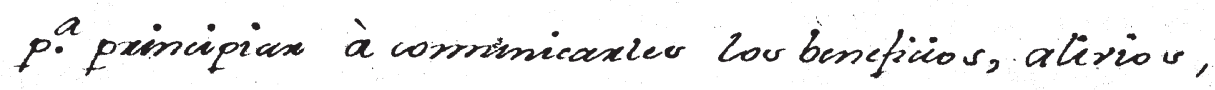

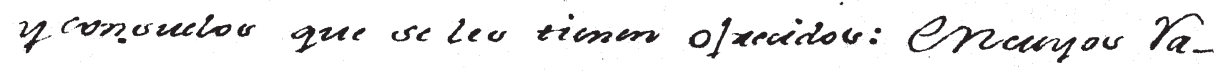

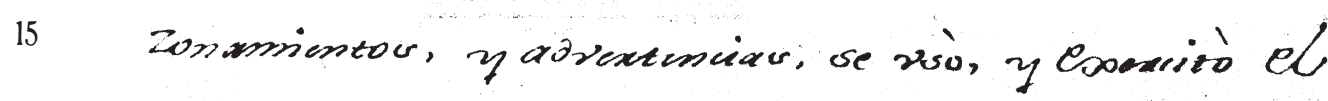
estió proprio à su Musticidad, havta que se Logio imponeales el acsunto, y xhas efectos ox piedad que lo promoneren. 'T eambien se les instò à quedecla. xasem su rerto amimo, yla rexdadexa inzencion re

20 Su remida, y rion con niosacxor: y es täl supuento, lov refexidor Capizaner, ylow omas Indiov avistont. 
[fol. 2r]

\{top margin center\} $\backslash 371$

\{left margin\} 140

por qualquiera termino; no se tuvo por conveniente

en inteligencia de los genios flexibles, y desconfiados de los

Yndios, y por que se podrà executàr en la ocasion de

su asiento formàl, y cierto, à el fin de là reduccion

que en ellos se pretende. Y à este efecto, se le previno

à los expresados Capitanes Chiquito, y Casaca

blanca, y à otros muchos Yndios de los principales, que

concurrieron tambien en la dicha mi tienda, à que

por el dia de mañana se junten, y pasen con migo,

y los Reverendos Padres Apostolicos, à el reconocimiento de las

elegidas Tierras; $\mathrm{y}$ se les persuadiò à que despues las

ocupasen en su pueblo de Tiendas, y formàl asiento;

para principiar à comunicarles los beneficios, alivios,

y consuelos que se les tienen ofrecidos: ENcuyos ra-

zonamientos, y advertencias, se usò, y exercitò el

estilo proprio à sua Rusticidàd, hasta que se logrò

imponerles del asunto, y de los efectos de piedàd que

lo promueven. Y tambien se les instò à que decla-

rasen su recto animo, y la verdadera intencion de

su venida, y union con nosotros: y en tàl supuesto,

los referidos Capitanes, y los demas Yndios asistentes 
Ohjexon, que lo practicaxian awi; como seles proponia, danso ariso anter à los que no extaren presenters p. $q$.

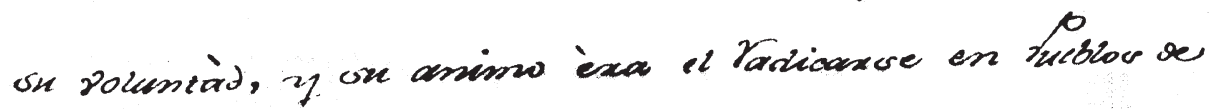

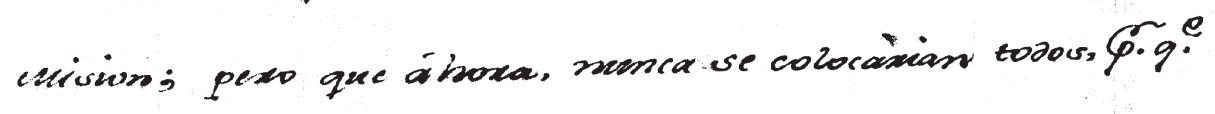

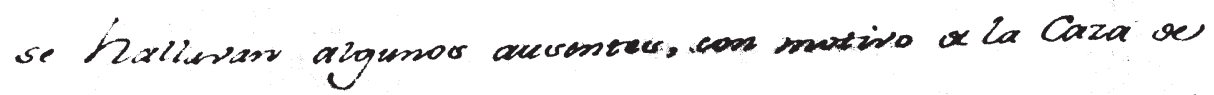
Sibolar, à que hacian maxchacis mechor a ellor desde el

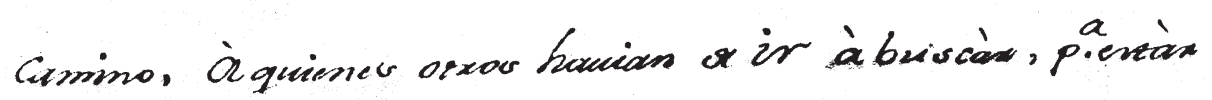

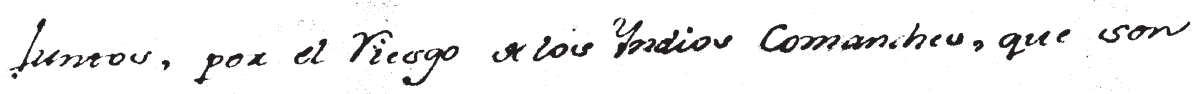

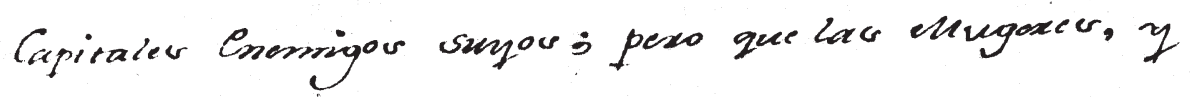

10 los evinos se congryanians enlos temmines que seles pxopuso. 21 dijexon tambien, que pox lo torante à el txarafo en que se espexistan los Indios of Lues ncievion. i. him niesto, no le poxian hacex epponto, pox que lo

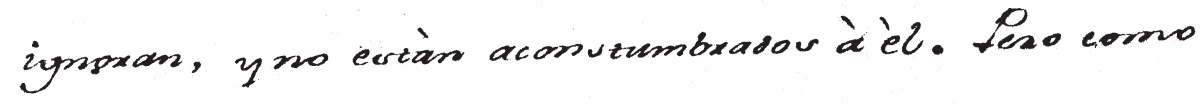

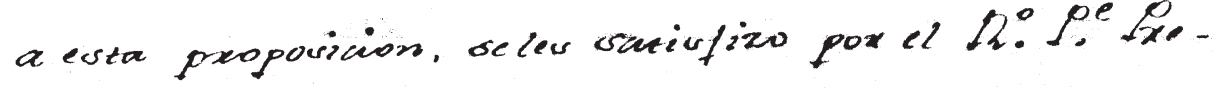
sibure, yderniar Lhelig. con los of uevimientour a comodidìd, y de sèx rmixador coms à rifips, y Yo les-impuse codo lo que el they les fasoxece, y amporar, quedaxon sin repaxs, y desnoutxason complacmia: y

20 el asiostin eldia de mainerna à el recomocinto $x$ lats

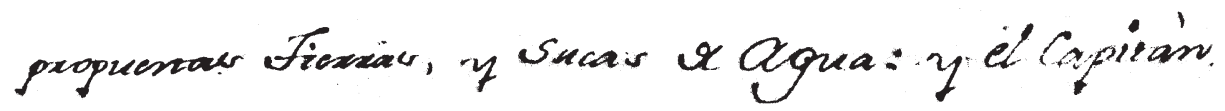




\section{[fol. 2v]}

\{top margin center\} $\backslash 372$

\{left margin\} 141

dijeron que lo practicarian asi; como se les proponia, dando aviso antes à los que no estavan presentes; por que su voluntàd, y su animo èra el radicarse en Pueblos de Mision; pero que á hora, nunca se colocàrian todos, por que se hallavan algunos ausentes, con motivo de la Caza de Sibolas, à que havian marchado muchos de ellos desde el camino, àquienes otros havian de ir à buscàr, para estàr juntos, por el Riesgo de los Yndios Comanches, que son Capitales enemigos suyos; pero que las Mugeres, y los Niños se congregarian en los termines que se les propuso. Y dijeron tambien, que por lo tocante à el travajo en que se exercitan los Yndios de las Misiones que hàn visto, no le podràn hacèr depronto, por que lo ignoran, y no estàn aconstumbrados à èl. Pero como à esta proposicion, se les satisfizo por el Reverendo Padre Presidente, y demàs Religiosos con los ofrecimientos de comodidàd, y de sèr mirados como à hijos, y Yo les impuse todo lo que el REY les favorece, y ampara, quedaron sin reparo, y demostraron complacencia: y el asistir el dia de mañana à el reconocimiento de las propuestas Tierras, y Sacas de Agua: y el Capitàn 
Spanish Colonial Documents Pertaining to Mission San Sabá

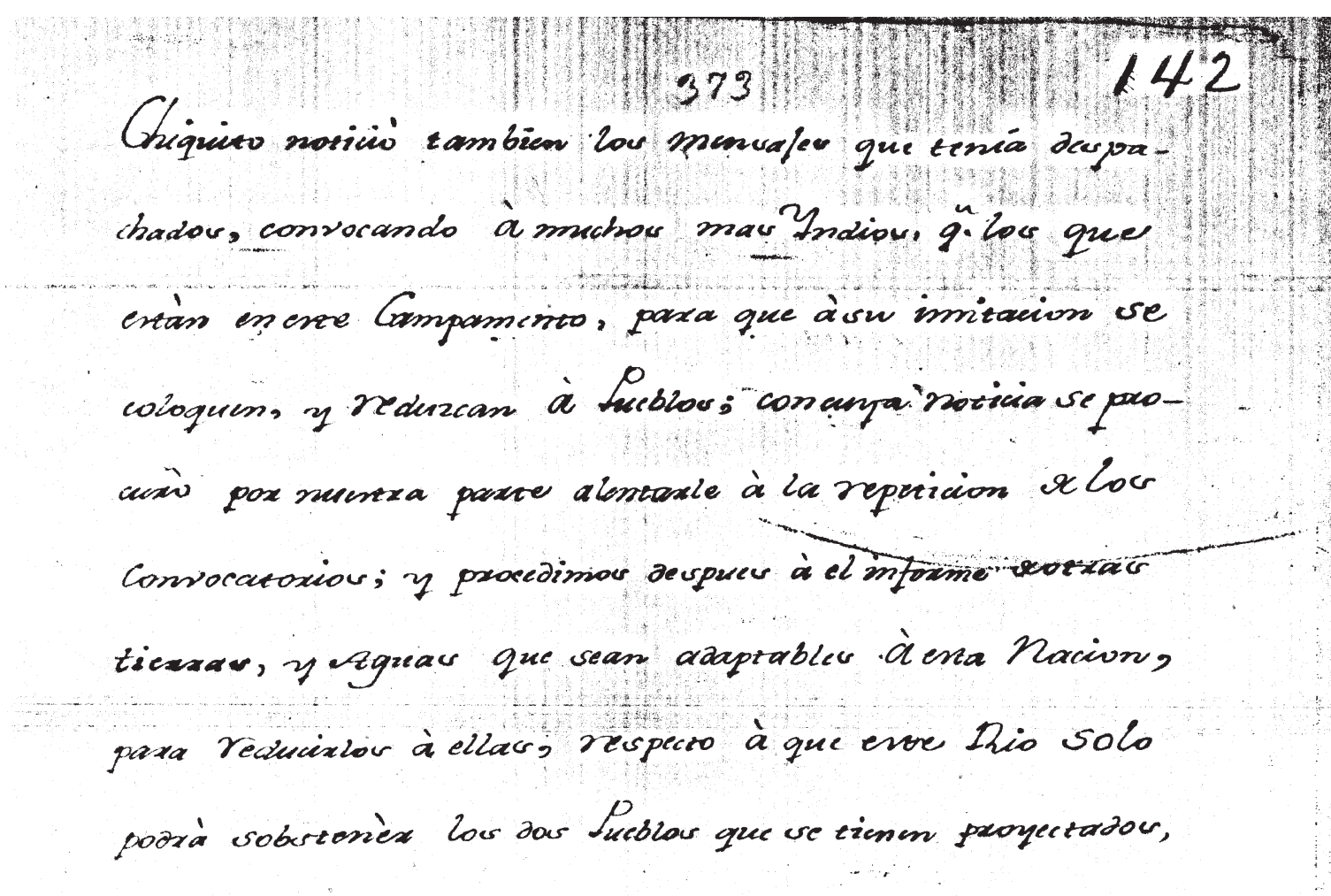

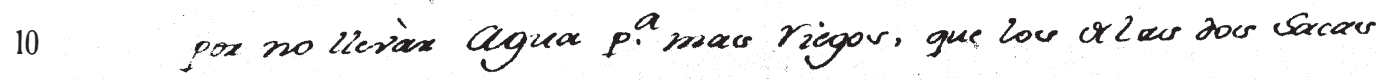

que se tienen reconocidas. Faunque bor bhvers. Capitanes,

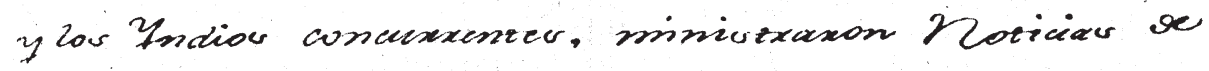

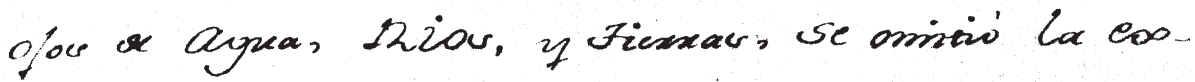

precsion enerza dilig. ni el procedè à orka sobse el

15

dermo, hasta que la pida, y demande la vxyencia,

If freccision conel concuaro, y pecindàd elou Zozd.

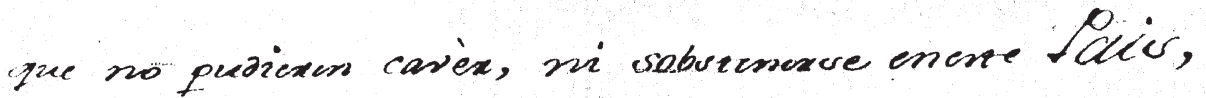

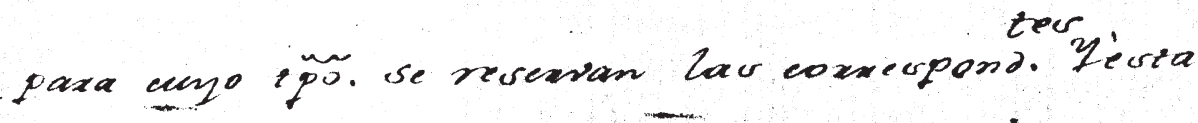

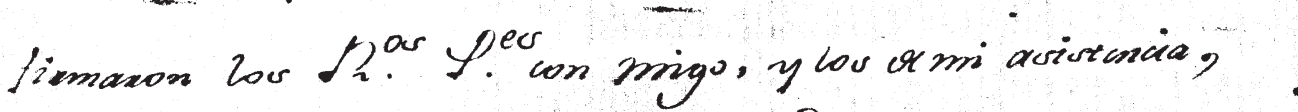

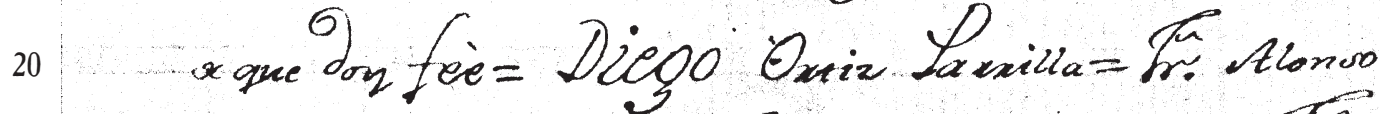

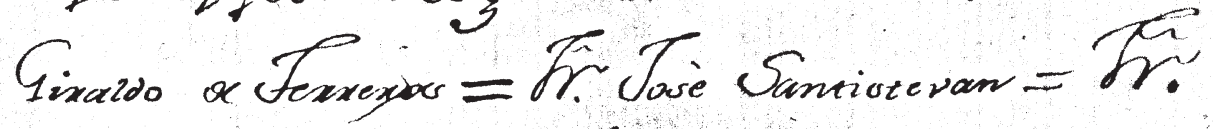


[fol. 3r]

\{top margin center\} $\backslash 373$

\{left margin 142

Chiquito noticiò tambien los mensajes que tenía despa-

chados, convocando à muchos mas Yndios, que los que

estàn en este Campamento, para que à su imitacion se

coloquen y reduzcan à Pueblos; con cuya noticia se pro-

curò por nuestra parte alentarle à la repeticion de los

convocatorios; y procedimos despues à el informe deotras

tierras, y Aguas que sean adaptables à esta Nacion,

para reducirlos à ellas, respecto à que este Rio solo

podrà sobstenèr los dos Pueblos que se tienen proyectados,

por no llevàr agua para mas riegos, que los de las dos sacas

que se tienen, reconocidas. Y aunque los dichos Capitanes,

y los Yndios concurrentes, ministraron Noticias de

ojos de agua, Rios, y Tierras, se omitiò la ex-

presion en esta diligencia ni el procedèr à otra sobre el

asunto, hasta que la pida, y demande la urgencia,

y precision con el concurso, y vecindàd de los Yndios

que no pudieren cavèr, ni sobstenerse en este Pais,

para cuyo tempo se reservan las correspondentes. Y èsta

firmaron los Reverendos Padres con migo, y los de mi asistencia,

de que doy feè = Diego Ortiz Parrilla = Fray Alonso

Giraldo de Terreros $=$ Fray Josè Santistevan $=$ Fray 
Spanish Colonial Documents Pertaining to Mission San Sabá

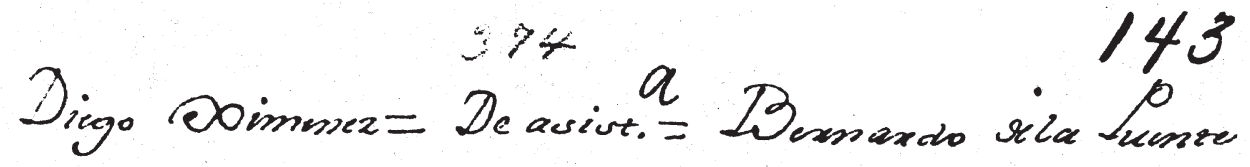

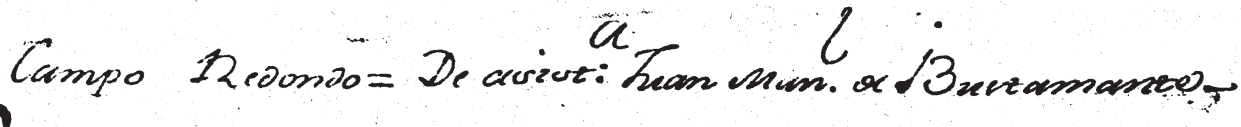


[fol. 3v]

\{top margin center\} $\backslash 374$

\{left margin\} 143

Diego Ximenez $=$ De assistenci $\mathrm{a}=$ Bernardo de la Puente

Campo Redondo = De assistencia Juan Manuel de Bustamante_ 


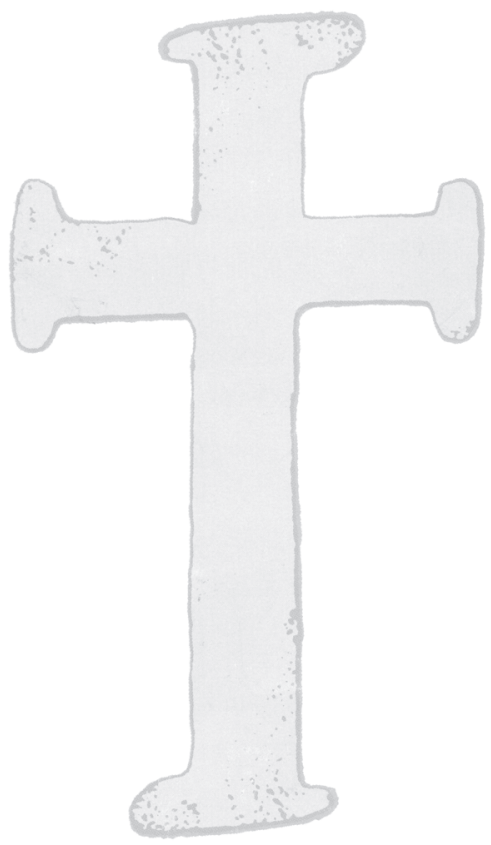




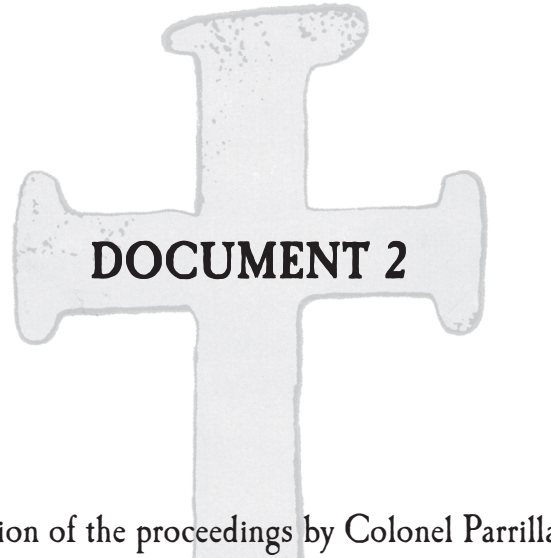

(with Fr. Alonso de Terreros, Fr. Santiesteban, Fr. Ximenez, Captain Chiquito)

May 26, 1757; 10 months before the attack

Center for American History, the University of Texas at Austin 


$$
27
$$

6

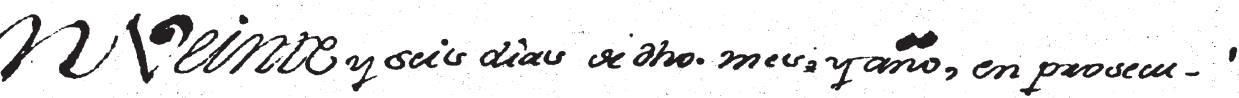
- cion ex extar dizigenciau, y en acencion à la presenida

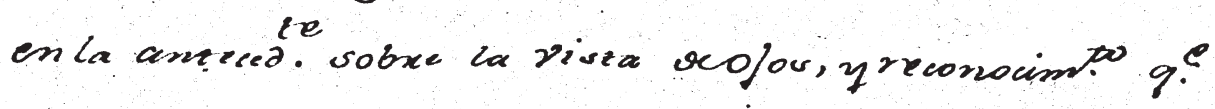
se pxopuso à los Inclior relav ticiexar queles eviàn.

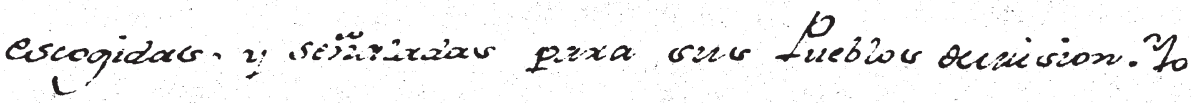

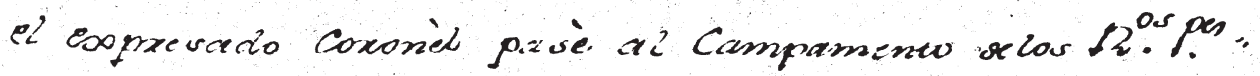

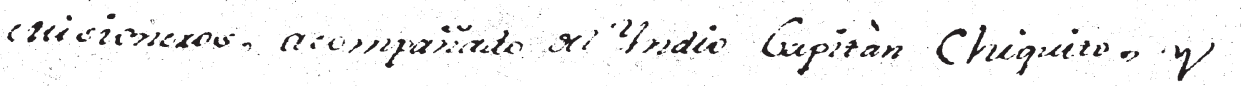

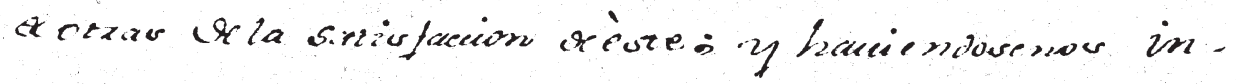

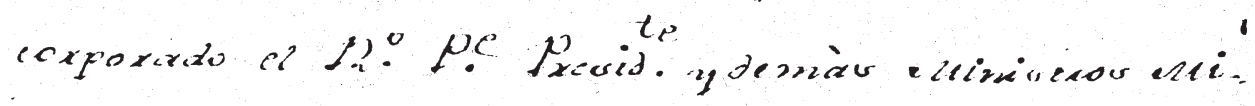

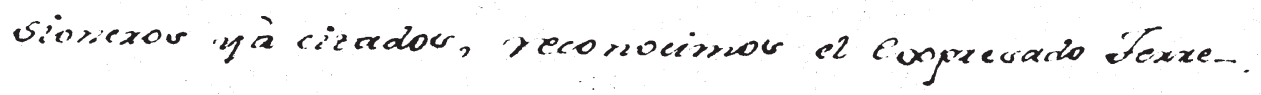
ro, exiside s. Lew impueso or Las cixcerrzancias es.

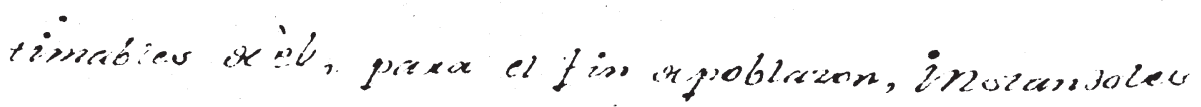

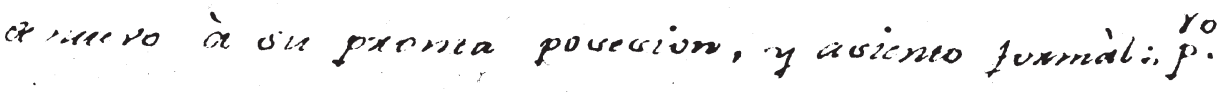

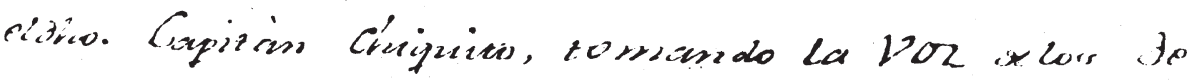

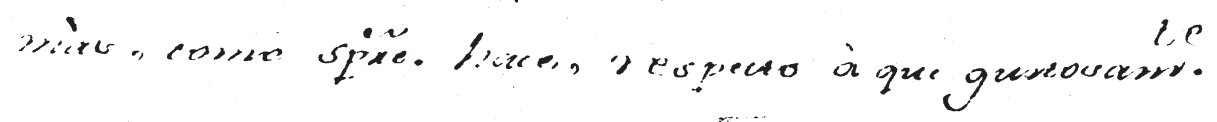

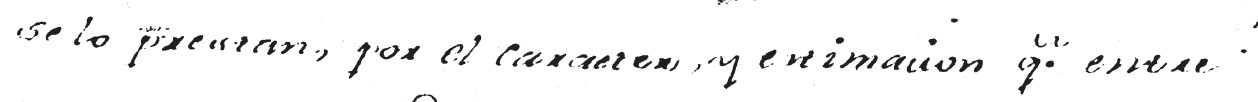

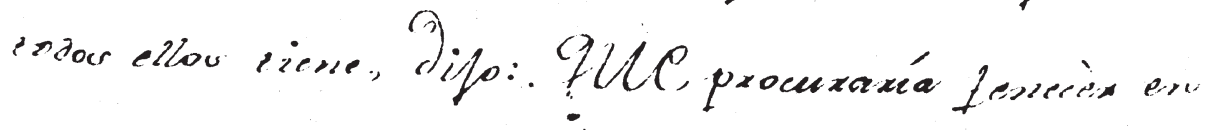

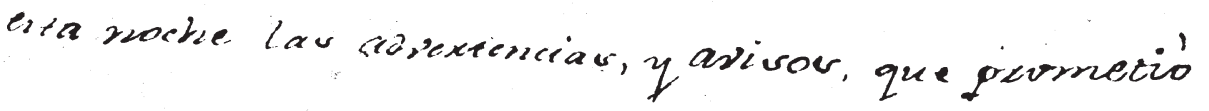


[fol. 1r]

\{top margin center\} $\backslash 374$

\{right margin\} 143

ENVEINTE y seis dias de dicho mes, y año, en prosecu-

cion de estas diligencias, $\mathrm{y}$ en atencion à la prevenida

en la antecedente sobre la vista deojos, y reconocimiento que

se propuso à los Yndios de las tierras que les estàn

escogidas, y señaladas para sus Pueblos demision. Yo

el expresado coronèl pasè al campamento de los Reverendos Padres

Misioneros, acompañado del Yndio Capitàn Chiquito, y

de otras de la satisfaccion de èste; $y$ haviendosenos in-

corporado el Reverendo Padre Presidente y demàs Ministros Mi-

sioneros yà citados, reconocimos el expresado Terre-

no, en donde se les impuso de las circunstancias es-

timables de èl, para el fin de poblazon, instandoles

de nuevo à su pronta posesion, y asiento formàl; primero

eldicho Capitàn Chiquito, tomando la Voz de los de-

màs, como sempre hace, respecto à que gustosamente

se lo prestan, por el caracter, y estimacion que entre

todos ellos tiene, dijo: QUE procuraría fenecèr en

esta noche las advertencias, y avisos, que prometiò 
Spanish Colonial Documents Pertaining to Mission San Sabá

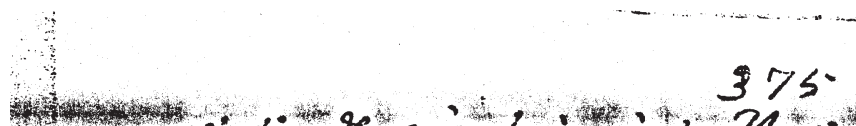

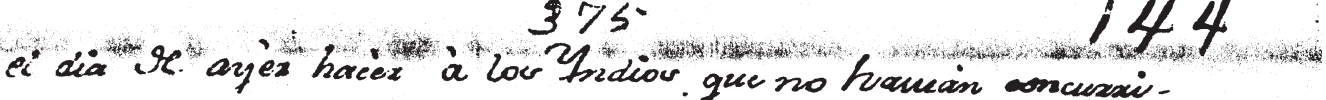

is con ellos, $72 i$ con hovotxos enez lanse güse pexcive

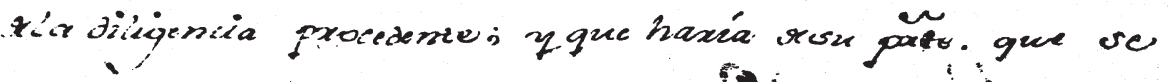

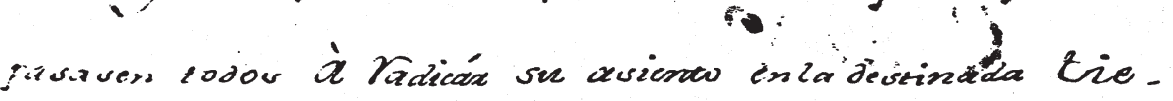

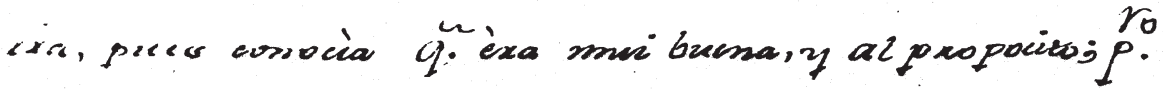

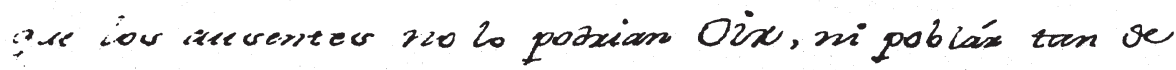

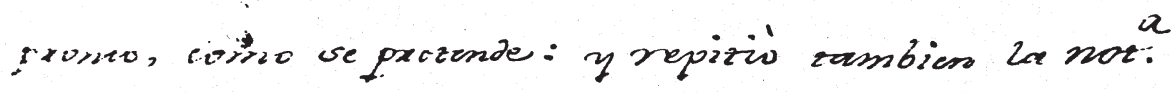

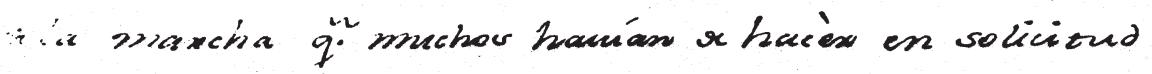

- izes Sibclas, y je oexas Cazar or que exzan recesitajg:

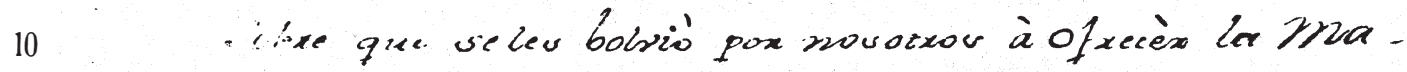

weernion otooos, sin que necesitasen of salix al monze,

$\therefore$ ier afianio one comodidader, y alivior con lav eos.

tresioneer que les hice, y queles paopusienom ioush.

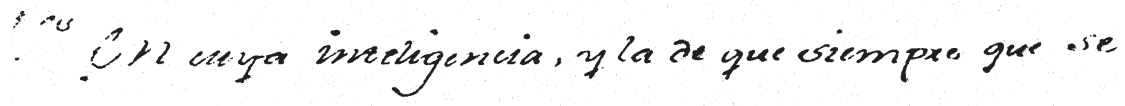

15

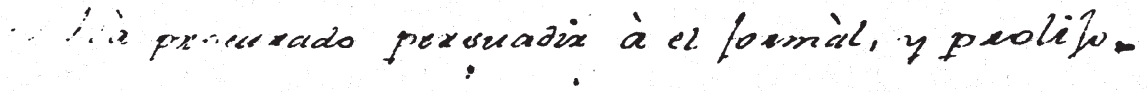

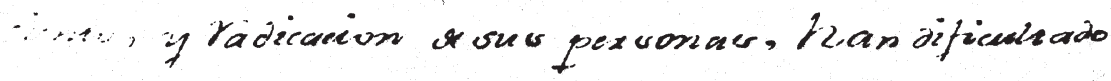

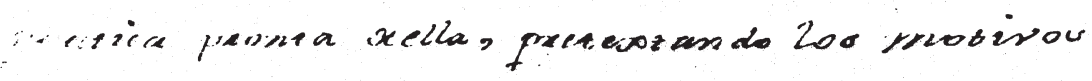

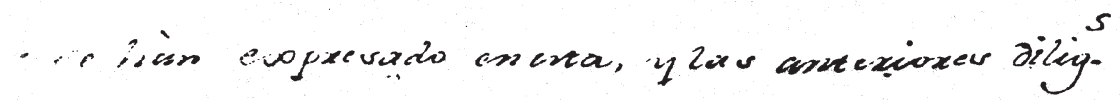

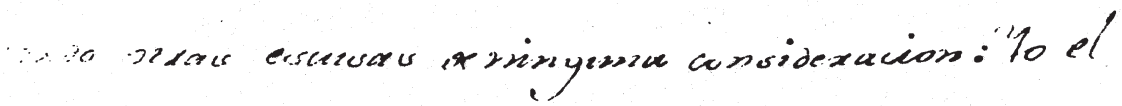

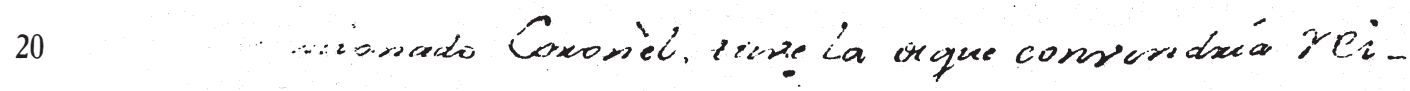

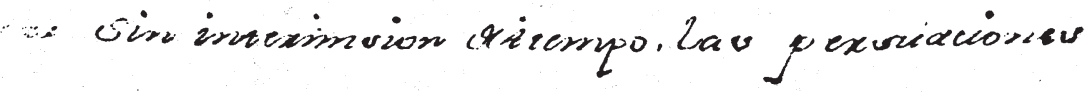


[fol. lv]

\{top margin center\} $\backslash 375$

\{right margin\} 144

el dia de ayèr hacèr à los Yndios que no havian (^con)curri-

do con ellos, Ni con nosotros en el lance que se percive

de la diligencia procedente; y que haría de su parte que se

pasasen todos à radicár su asiento en la destinada tie-

rra, pues conocìa que èra mui buena, y al proposito; primero

que los ausentes no lo podrian Oir, ni poblár tan de

pronto, como se pretende: y repitiò tambien la noticia

de la marcha que muchos havían de hacèr en solicitud

de las Sibolas, y de otras cazas de que estàn necesitados:

sobre que se les bolviò por nosotros à ofrecèr la ma-

nutencion de todos, sin que necesitasen de salir al Monte,

y se les afianzò sus comodidades, y alivios con las ex-

presiones que les hice, y que les propusieron los Reverendos

Padres En cuya inteligencia, y la de que siempre que se

les hà procurado persuadir à el formàl, y prolijo,

asiento, y radicacion de sus personas, han dificultado

la practica pronta de ella, pretextando los motivos

que se hàn expresado en esta, y las anteriores diligencias

y dando otras escusas de ninguna consideracion: Yo el

mencionado Coronèl, tuve la de que convendría rei-

teràr sin intermision de tiempo las persuaciones 


\section{$3 \%$}

125

inas eficuzer, no defordoles olo mano, ni omitiondo can eíow ocasion, ni lance pax aprorechain a zodaw hoxace

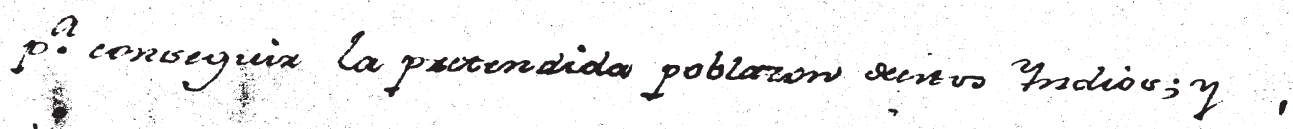
ciene efecto propuse à lov nefexidor hos pes elque ve

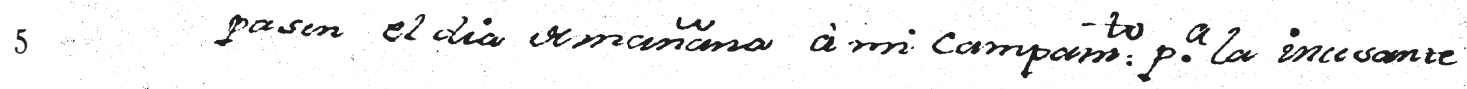
zaxe e postolica, respecto à eniax acampados en ere $\therefore$ inediscion los findios, qu se wixan tan indextex... mindatos, $y$, sin accion ento mimo $q^{\circ}$ ofxcan, yque.

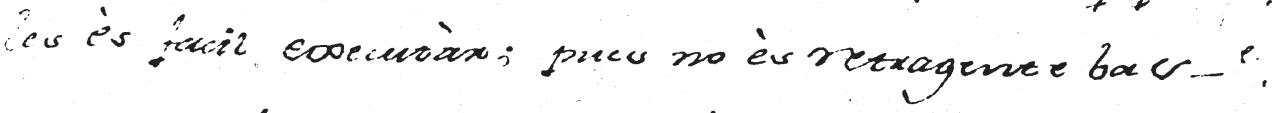

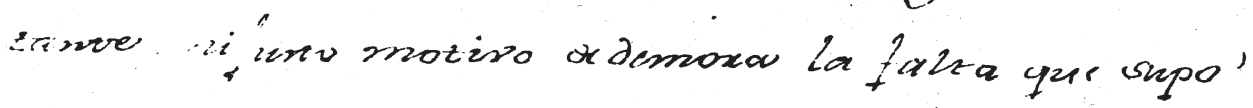

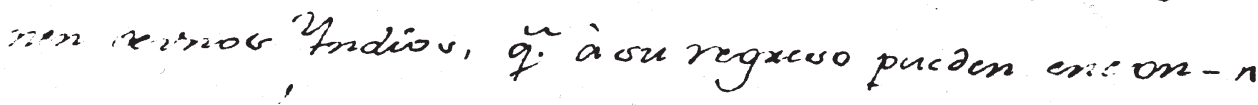

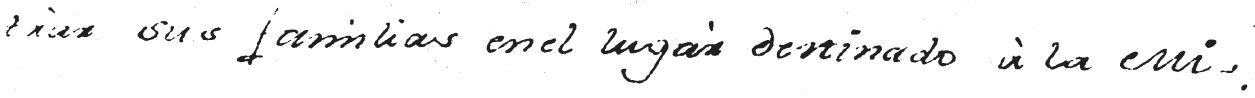

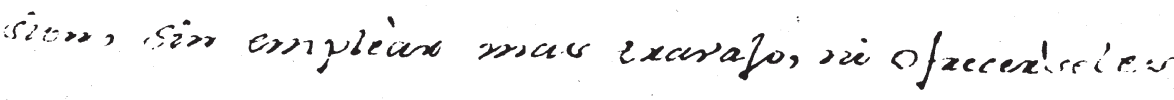

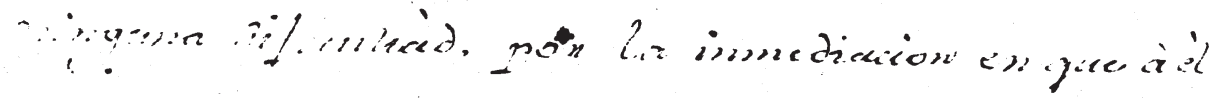

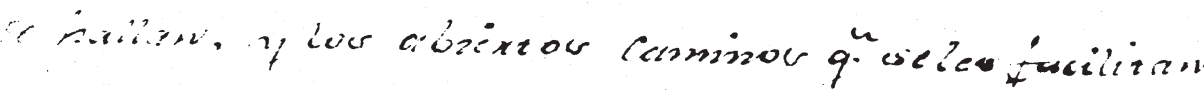

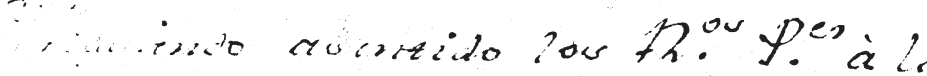

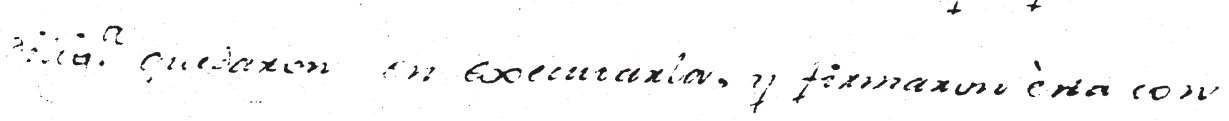

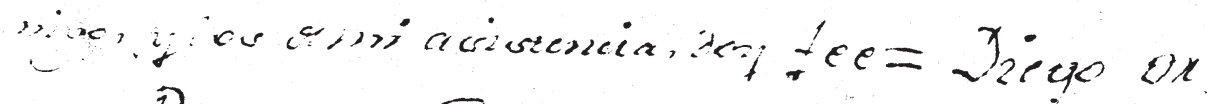

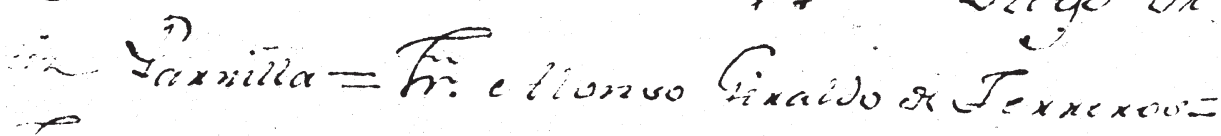

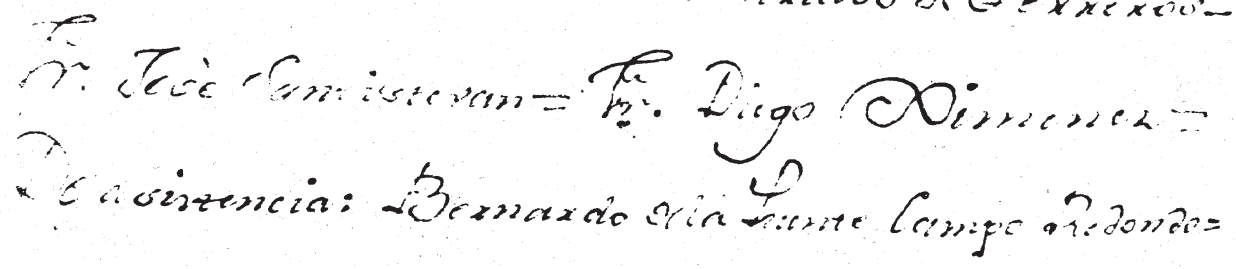


[fol. 2r]

\{top margin center\} $\backslash 376$

\{right margin\} 145

mas eficazes, no dejandoles de la mano, ni omitiendo con

ellos ocasion, ni lance por aprovechàr à todas horas

para conseguir la pretendida poblazon deestos Yndios; y

à este efecto propuse à los referidos Reverendos Padres el que se

pasen el dia de mañana à mi campamento: para la incesante

tare [tarea] Apostolica, respecto à estàr acampados en su

inmediacion los Yndios, que se miran tan inderter-

minados, y sin accion en lo mismo que ofrecen, y que

les ès facil executàr; pues no ès retragente bas-

tante ni justo motivo de demora la falta que supo-

nen de unos Yndios, que à su regreso pueden encon-

tràr sus familias en el lugàr destinado à la Mi-

sion, sin emplèar mas travajo, ni ofrecerseles

ninguna dificultàd, por la inmediacion en que à èl

se hallan, y los abiertos caminos que se les facilitan.

Haviendo asentido los Reverendos Padres à la propuesta

diligencia quedaron en executarla, y firmaron èsta con

migo, $\mathrm{y}$ los de mi asistencia, doy fee $=$ Diego Or

tiz Parrilla = Fray Alonso Giraldo de Terreros=

Fray Josè Santistevan= Fray Diego Ximenez=

De asistencia : Bernardo dela Puente Campo Redondo= 
Spanish Colonial Documents Pertaining to Mission San Sabá

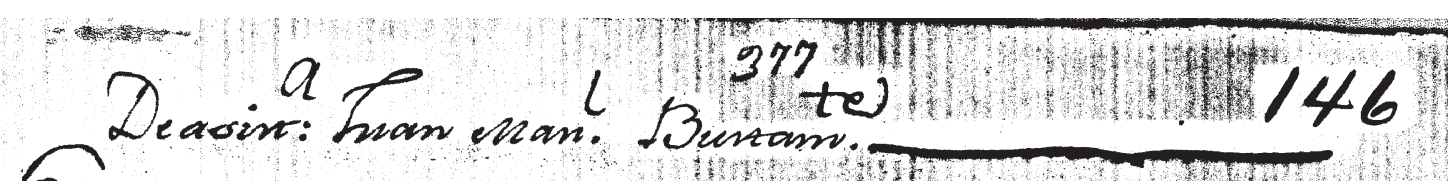


Document 2

\{fol. 2v]

\{top margin center $\} \backslash 377$

\{right margin\} 146

De asistencia: Juan Manuel Bustamante. 


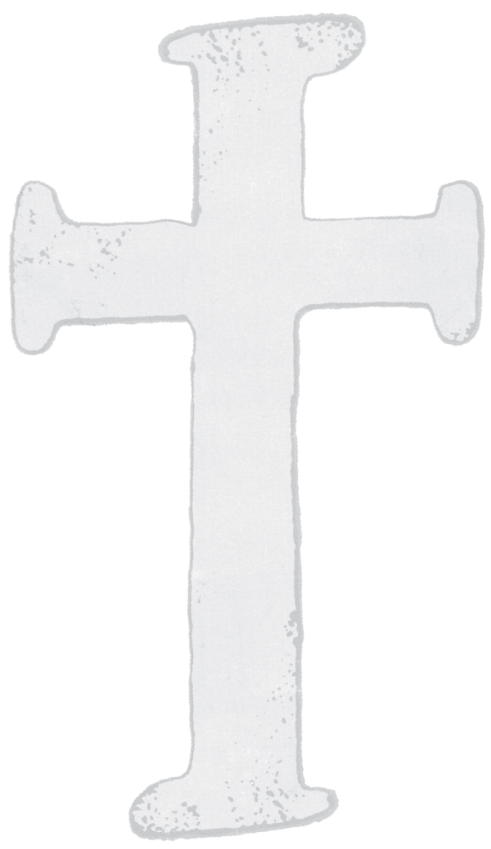




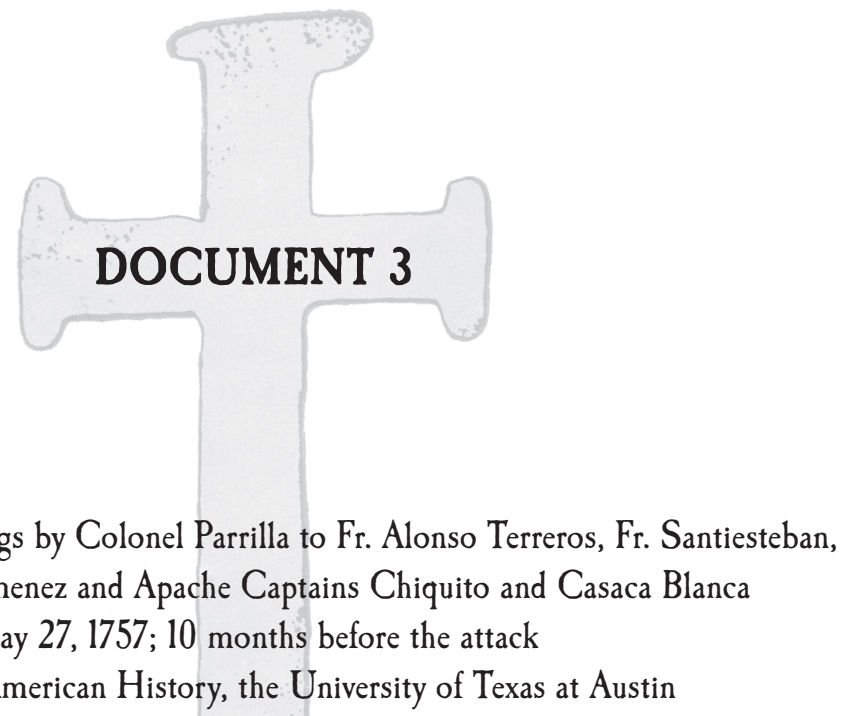

Center for American History, the University of Texas at Austin 
Spanish Colonial Documents Pertaining to Mission San Sabá

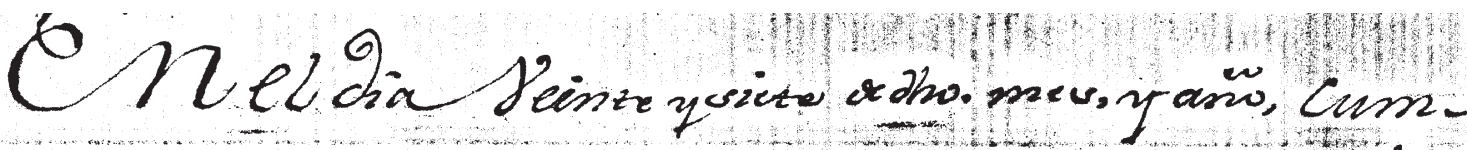
te

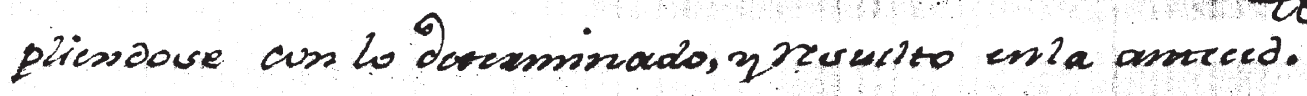

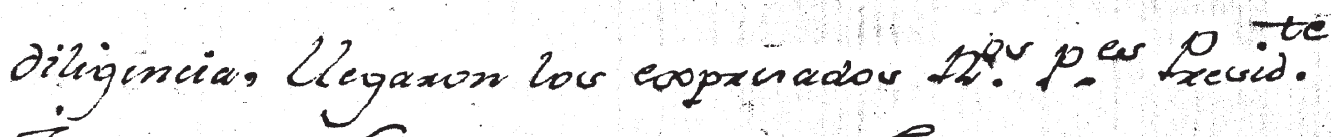

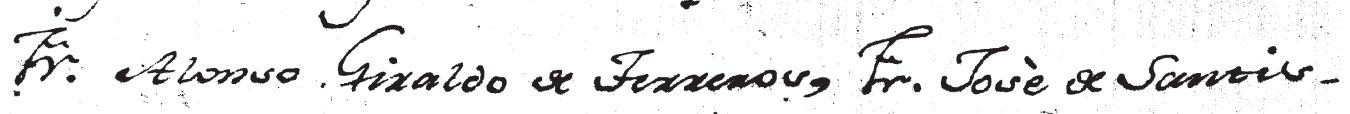
zepan, y i⿱⺈. Diego Dimenes à incoxpoxaxere conmigo el mencionado Coxonil, camo à lav siver ymedia de ía manumas 2 haciendonor fumado en mi Fienda

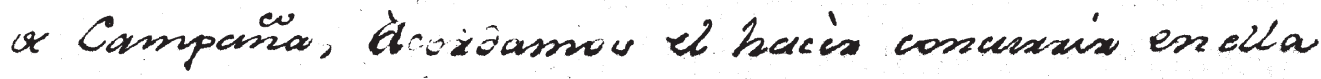

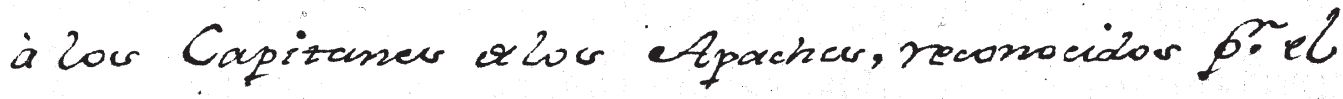
Giquitu, y Ga sacablanca, y à oxwor or tos pxales.

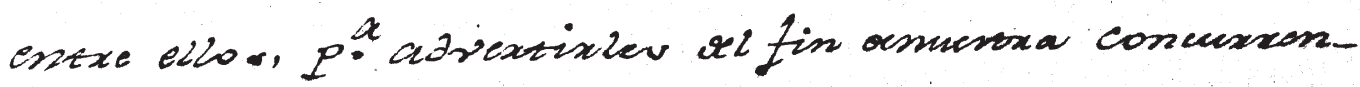
cia, ylus facilur medios quec tionen $p$ a no dizatax su conzpegacions à lour nivioner que tïness of xecizavi ytambien quanto conducer à rl Desengano, de

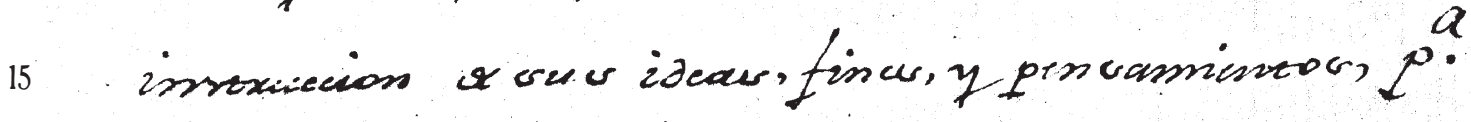
que en incelig. are tods ve adapton prosidencias, y

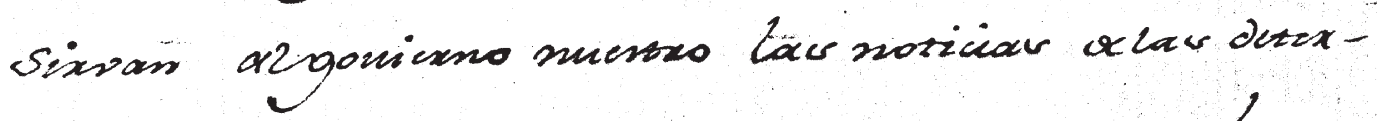

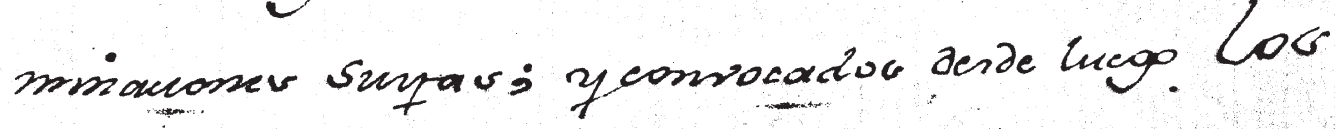
refexidor Zndior, que se funtaxon al pximex arijo 20 que selev dió pox medio ol Indio Intexp.Lamads

44 
[fol. 1r]

\{top margin center\} \377

\{right margin\} 146

EN el dia Veinte y siete de dicho mes, y año, cum-

pliendose con lo determinado, y resuelto en la antecedente

diligencia, llegaron los expresados Reverendos Padres Presidente

Fray Alonso Giraldo de Terreros, Fray Josè de Santis-

tevan, y Fray Diego Ximenez à incorporarse conmigo

el mencionado Coronèl, como à las siete y media de

la mañana; y haviendonos juntado en mi Tienda

de Campaña, àcordamos el hacèr concurrir en ella

à los Capitanes de los Apaches, reconocidos por el

Chiquito, y Casacablanca, y à otros de los principales

entre ellos, para advertirles del fin denuestra concurren-

cia, y los faciles medios que tienen para no dilatár

su congregacion à las Misiones que tienen ofreci-

das; y tambien quanto conduce à el desengaño, è

instruccion de sus ideas, fines, y pensamientos, para

que en inteligencia de todo se adapten providencias, y

sirvan al govierno nuestro las noticias de las deter-

minaciones suyas; y convocados desde luego los

referidos Yndios, que se juntaron al primèr aviso

que se les diò por medio del Yndio Interprete llamado 
Spanish Colonial Documents Pertaining to Mission San Sabá

378

147

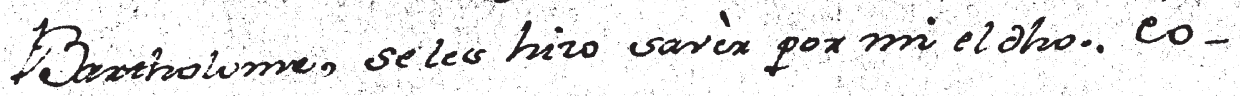

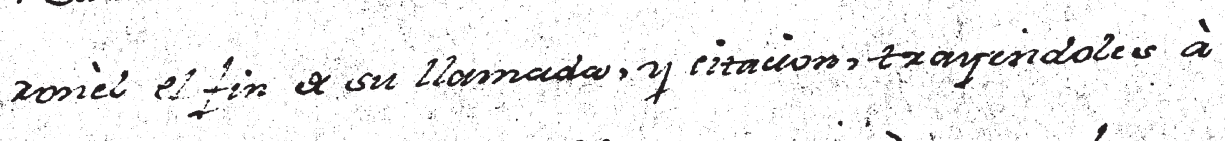
uz memoxia Jesda que hiüenon aminat, pax, y buena

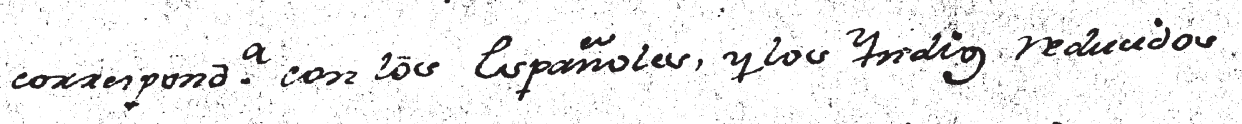

5

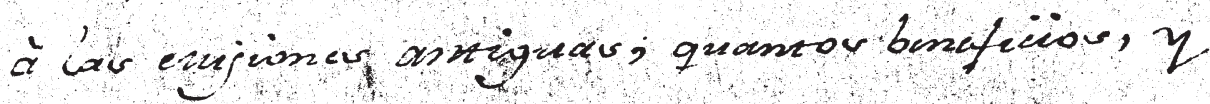

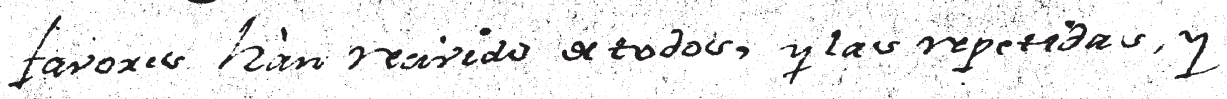

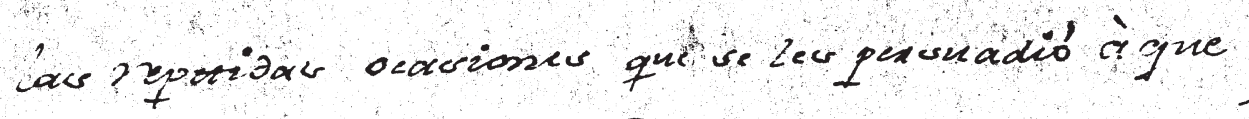
diozazasen la pexdadexa fiy que noworwe pafecra.

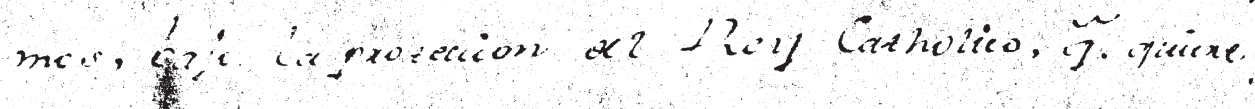
10

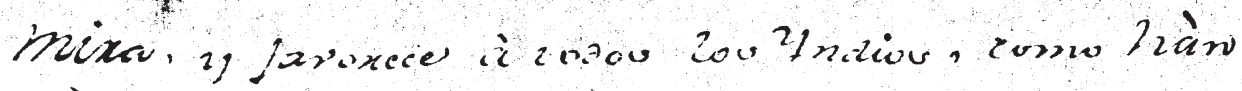

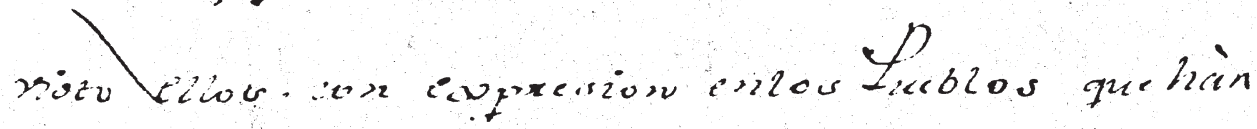

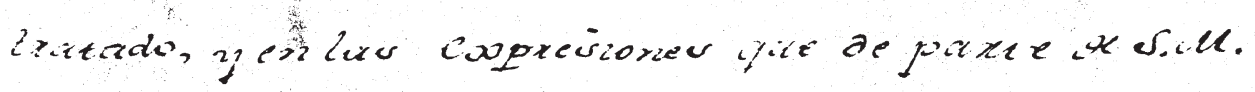

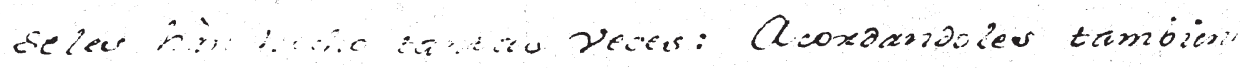

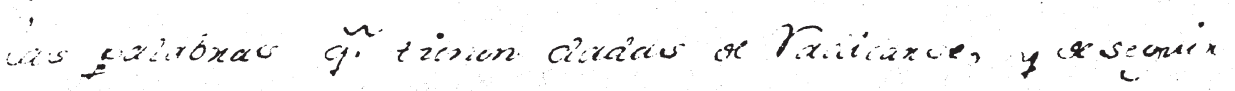
15

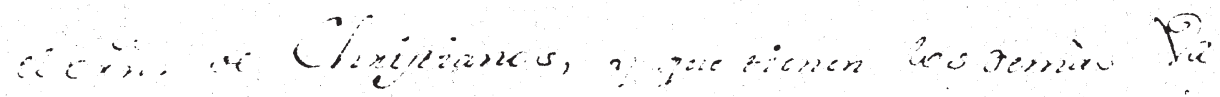

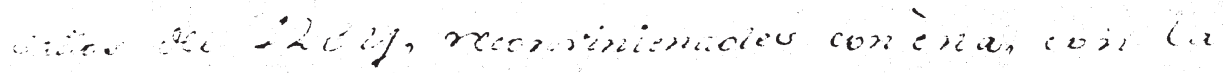

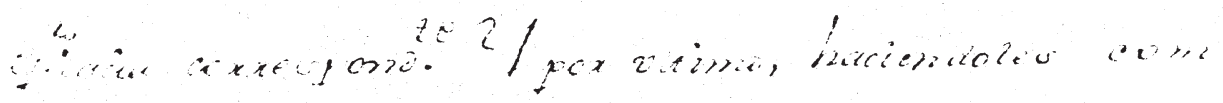

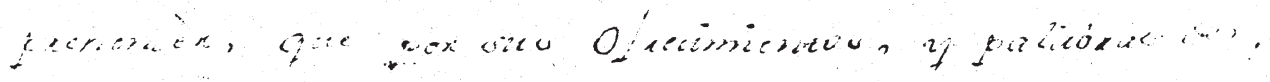

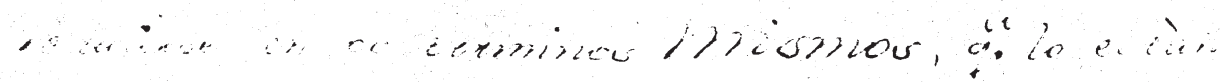

20

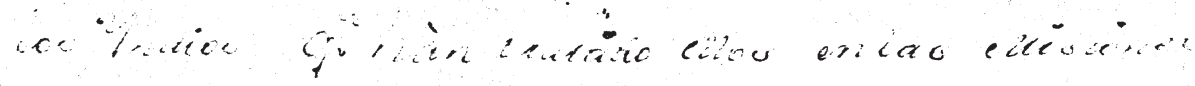

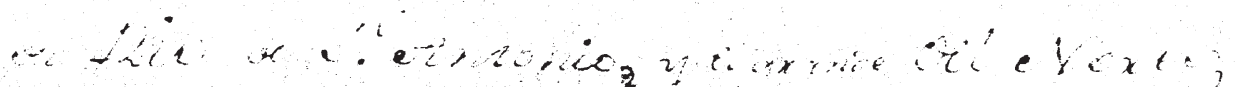

46 
[fol. lv]

\{top margin center $\} \backslash 378$

\{right margin\} 147

Bartholome, se les hizo savèr por mi el dicho, co-

ronèl el fin de su llamada, y citacion, trayendoles à

la memoria desde que hicieron amistad, paz, y buena

correspondencia con los Españoles, y los Yndios reducidos

à las Misiones antiguas; quantos beneficios, y

favores hàn recivido de todos, $\mathrm{y}$ las repetidas, $\mathrm{y}$

las repetidas ocasiones que se les persuadió à que

abrazasen la verdadera Ley que nosotros profesa-

mos, bajo la proteccion del Rey Catholico, que quiere,

mira, y favorece à todos los Yndios, como hàn

visto ellos con expresion en los Pueblos que hàn

tratado, y en las expresiones que de parte de Su Magestad

se les hàn hecho tantas veces: acordandoles tambien

las palabras que tienen dadas de radicarse, y de seguir

el orden de Christianos, y que tienen los demàs Va-

sallos del REY, reconviniendoles con èsta con la

eficacia correspondente. Y por ultimo, haciendoles com-

prehendèr, que por sus ofrecimientos, y palabras de

reducirse en los terminos MISMOS, que lo estàn

los Yndios que hàn tratado ellos en las Misiones

del Rio de San Antonio, y el grande del Norte; 
Spanish Colonial Documents Pertaining to Mission San Sabá

donzo tanzau Ocasiones hain enaá, setomaxon pop,

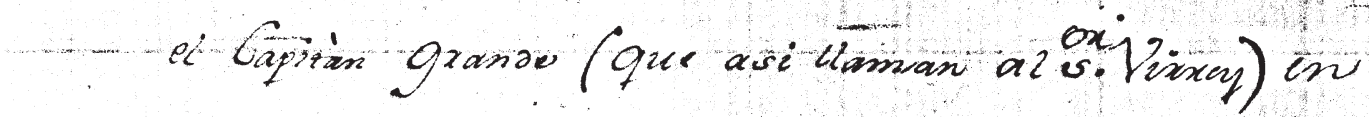

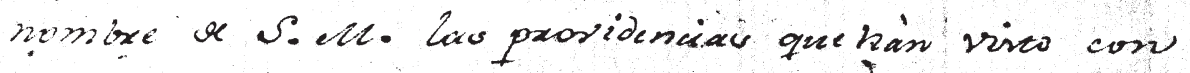

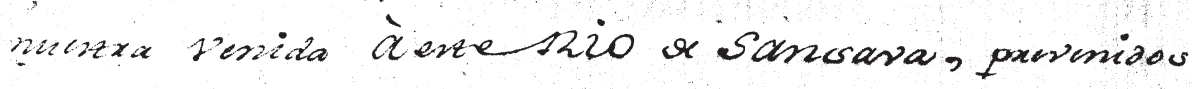

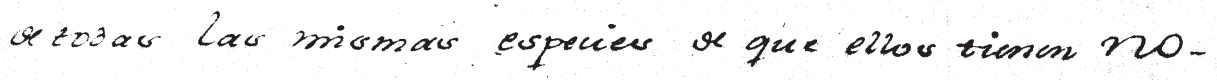

ticia, yque ve conumen, I gastan conlos Yradio re-

cividos à la viala Goxiriza y el Axmamento or uncido

Pres. paxa prozefexizor, ampaxaxios, yofendéx el texxeno

\&los Enemigor que le intentasen hasizlizax. y hacex-

10 les zaño à elzos, como sus hasizadoxes contodo el

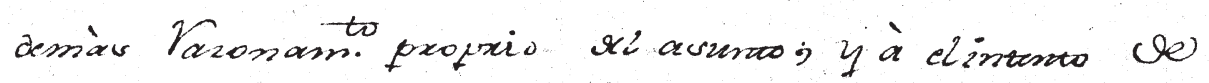

que tomen ia resolucion expoblá el texeno destinado

à exisiones: conno debermos espexan que haxa, pox te-

- zicio as pxomezido. Y añalidar à entas, ozxar

15 suapes intimacimes a ahos. L20s Les sobre elzzare

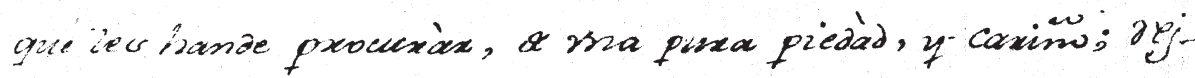

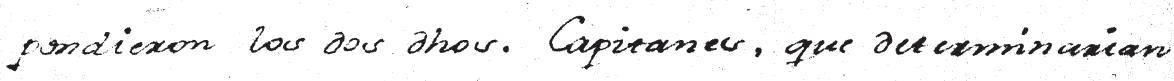

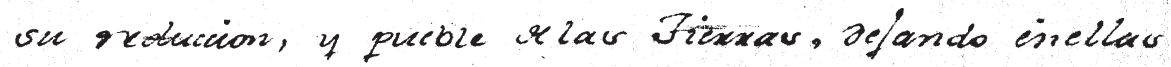

à Las farritias ennugeses, y niños, por que elzor que-

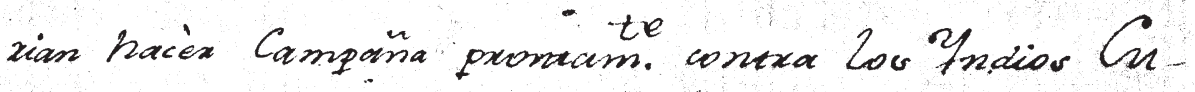

manches, y que pedian, gre paxa ella, seles ausoiliase 
[fol. 2r]

\{top margin center\} $\backslash 379$

\{right margin\} 148

donde tantas ocasiones hàn estado, se tomaron por

el Capitàn Grande (que asi llaman al Señor Virrey) en

nombre de Su Magestad las providencias que hàn visto con

nuestra venida à este Rio de Sansava, prevenidos

de todas las mismas especies de que ellos tienen no-

ticia, y que se consumen, y gastan con los Yndios, re-

cividos à la vida Christiana y el armamento de un crecido

Presidio para protejerlos, ampararlos, y defendèr el terreno

de los enemigos que le intentasen Hostilizàr, y hacer-

les daño à ellos, como sus havitadores, con todo el

demàs razonamento proprio del asunto; y à el intento de

que tomen la resolucion de poblàr el terreno destinado

à Misiones: como debemos esperàr que harà, por te-

nerlo asi prometido. $Y$ añadidas à estas, otras

suaves intimaciones de dichos Reverendos Padres sobre el trato

que les hande procuràr, de una pura piedàd, y cariño; res-

pondieron los dos dichos Capitanes, que determinarian

su reduccion, y pueble de las Tierras, dejando en ellas

à las familias de Mugeres, y Niños, por que ellos que-

rian hacèr Campaña prontamente contra los Yndios $\mathrm{Cu}$ -

manches, y que pedian, que para ella, se les auxiliase

$[\ldots]$ 
Spanish Colonial Documents Pertaining to Mission San Sabá

$$
381
$$

149

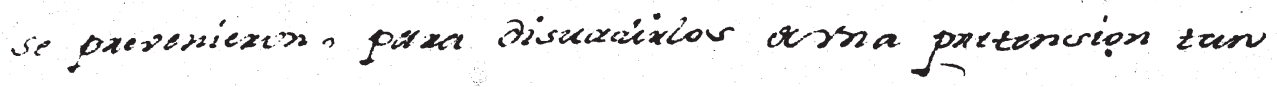

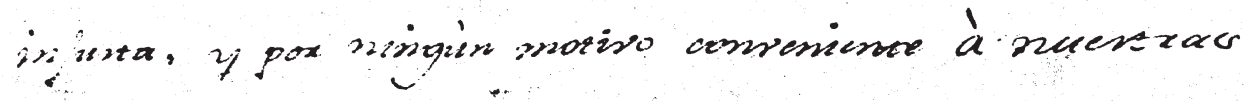
etumar. 'I pox tim, se resolvio, desconfiando aun $v$

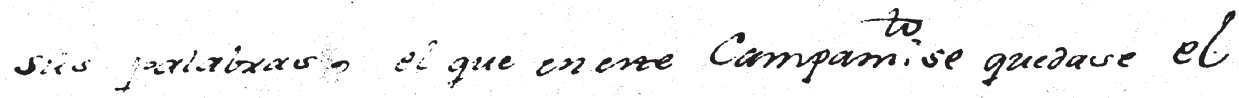

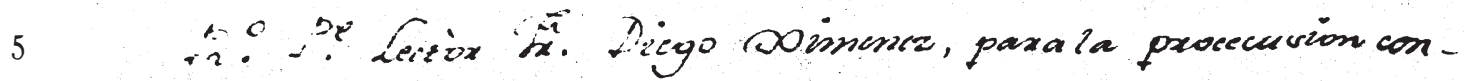

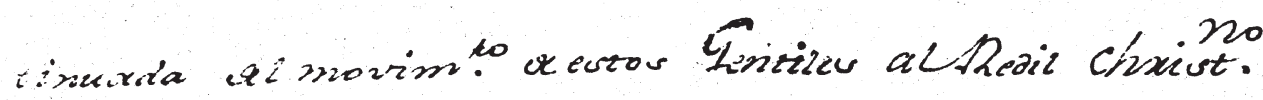

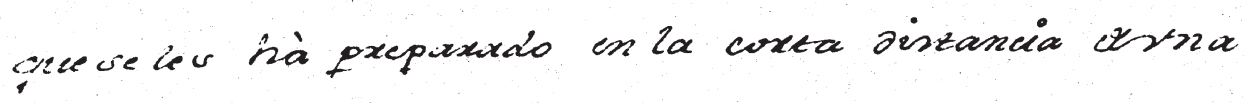
ievera ymeitix Haonae se hallan ellos acompañidor,

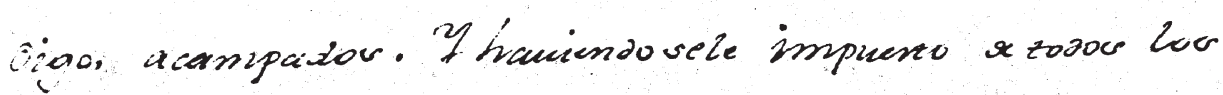

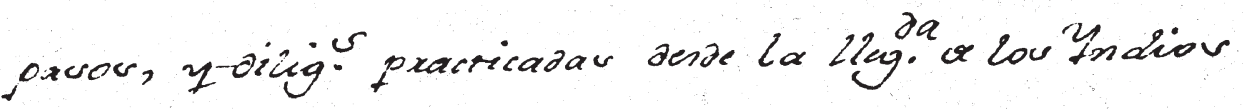

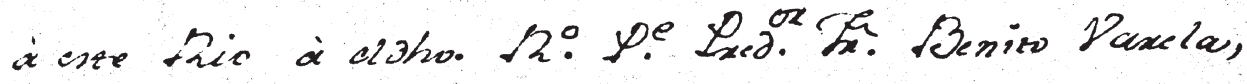

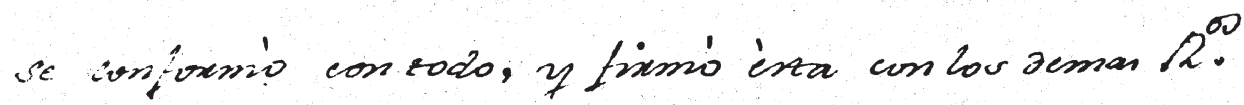

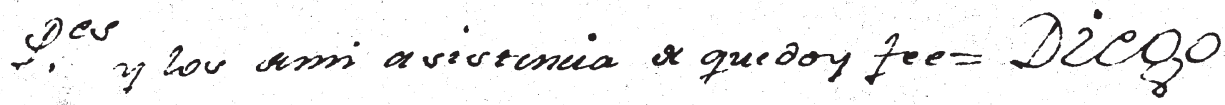

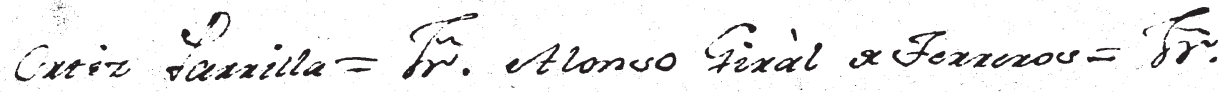

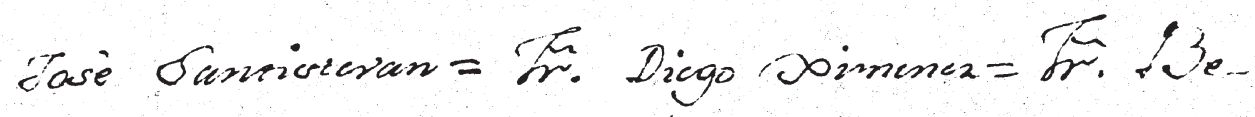

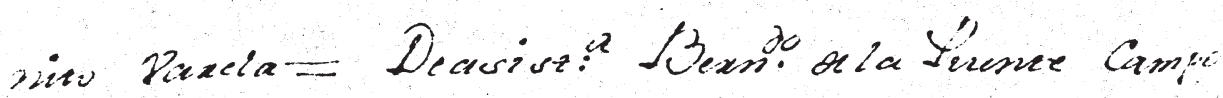

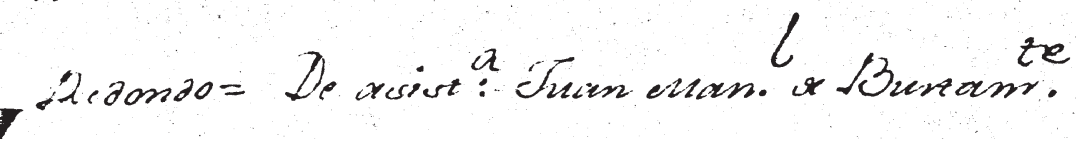




\section{[fol. 2v]}

\{top margin center\} $\backslash 381$

\{right margin\} 149

se prevenieron, para disuadirlos de una pretension tan

injusta, y por ningùn motivo conveniente à nuestras

Armas. Y por fin se resolviò, desconfiando aun de

sus palabras, el que en este Campamento se quedase el

Reverendo Padre Lectòr Fray Diego Ximenez, para la procecusion con-

tinuada del movimento de estos Gentiles al Redil Christiano

que se les hà preparado en la corta distancia de una

legua, y media de donde se hallan ellos acompañados,

digo, acampados. Y haviendosele impuesto de todos los

pasos, y diligencias practicadas desde la llegada de los Yndios

à este Rio à el dicho Reverendo Padre Predicador Fray Benito Varela, se conformò con todo, y firmò èsta con los demas Reverendos

Padres y los demi asistencia de que doy fee = Diego

Ortiz Parrilla $=$ Fray Alonso Giràl de Terreros $=$ Fray

Josè Santistevan= Fray Diego Ximenez= Fray Be-

nito Varela= De asistencia: Bernardo de la Puente Campo

Redondo= De asistencia : Juan Manuel de Bustamante 


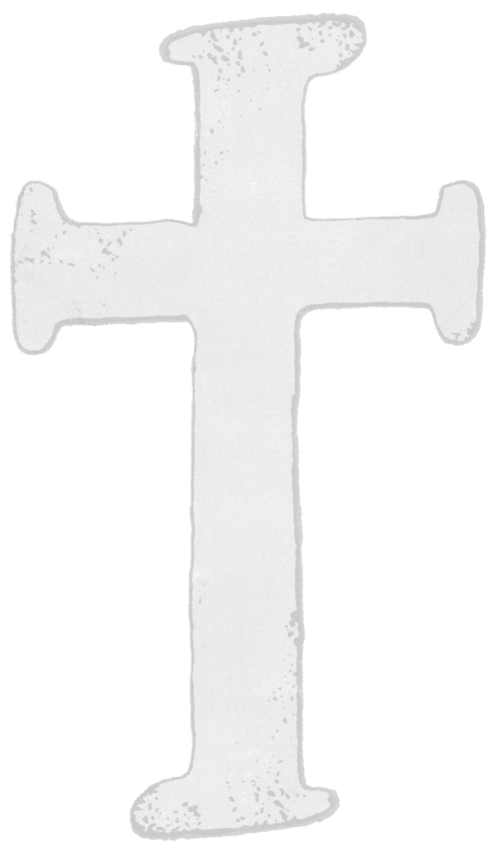




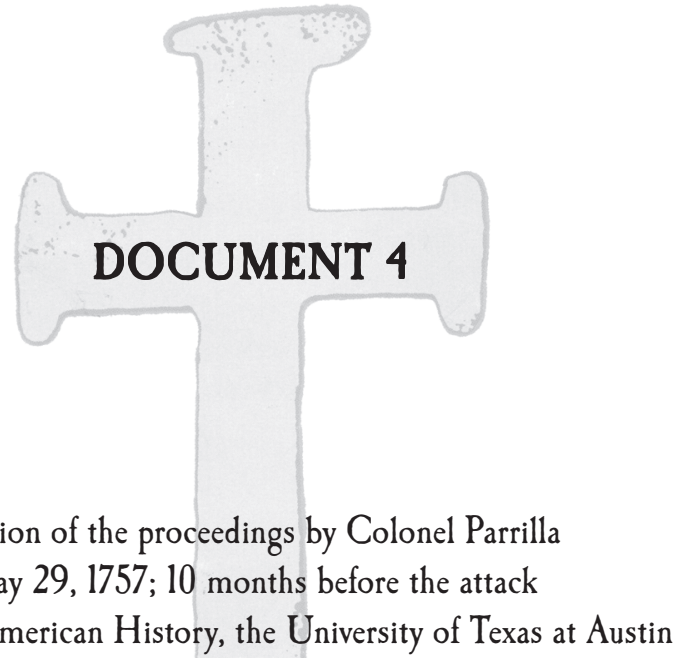


Spanish Colonial Documents Pertaining to Mission San Saba

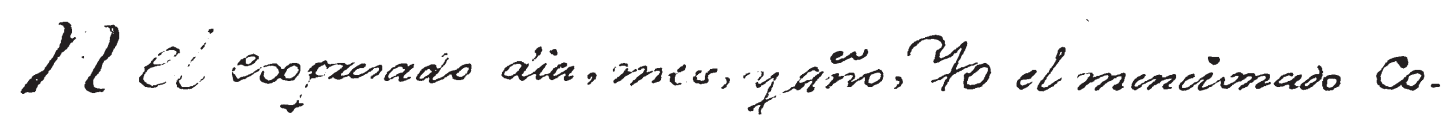

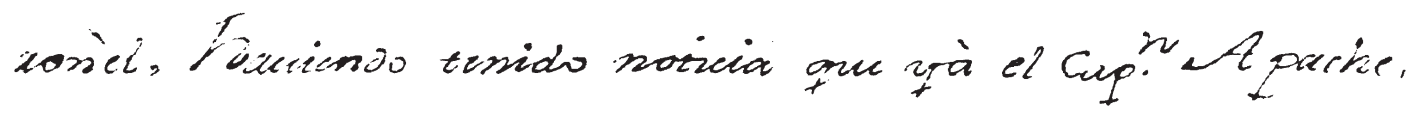

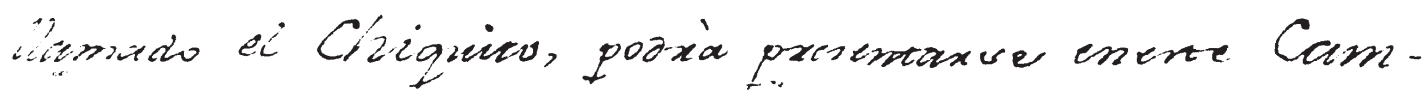

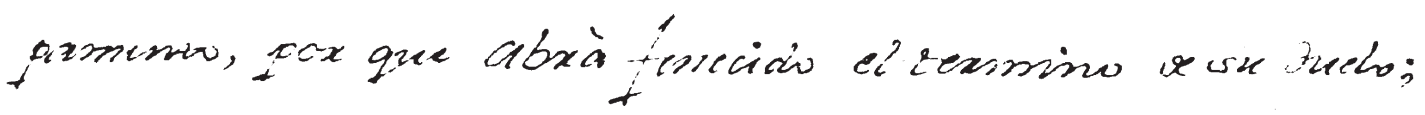

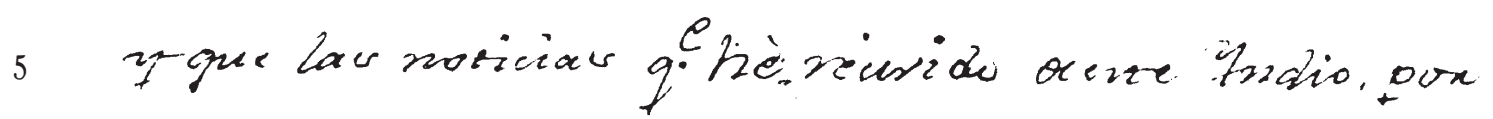

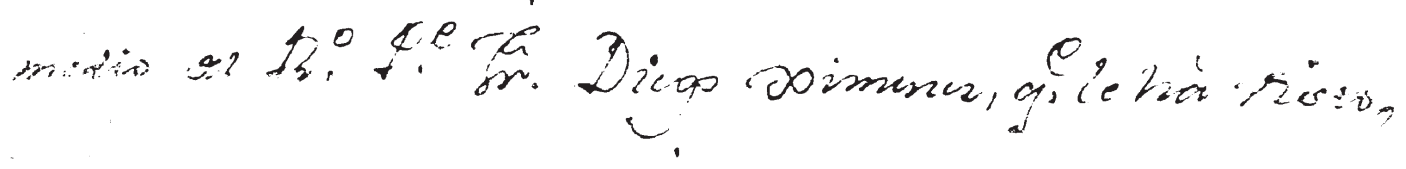

54 
[fol. lr]

\{top margin center\} \393

\{right margin\} 161

EN el expresado dia, mes, y año, Yo el mencionado coronèl, haviendo tenido noticia que yà el Capitàn Apache, llamado el Chiquito, podrà presentarse en este Campamento, por que abrà fenecido el termino de su duelo; y que las noticias que hè recivido de este Yndio, por medio del Reverendo Padre Fray Diego Ximenez, que le hà visto, 
Spanish Colonial Documents Pertaining to Mission San Sabá

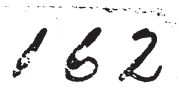

y soicicita do rypoticitis recer, pexusuaden à exeex, $g^{?}$ su

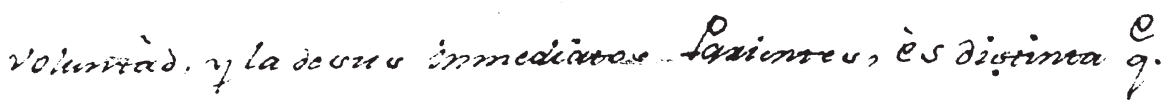

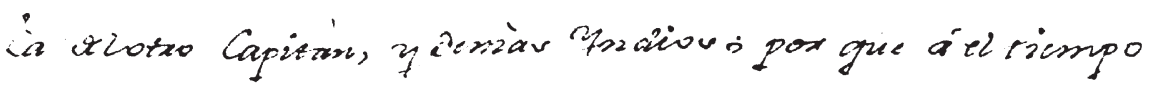

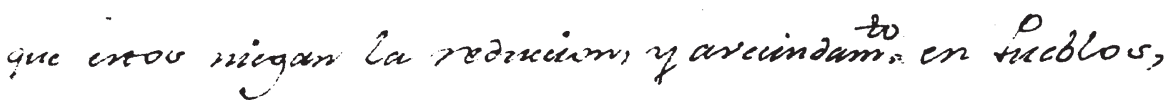

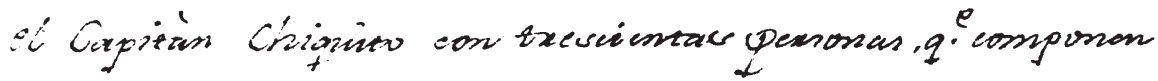
cas 32 . Fiendas et cornoañ que timen funto à la vinga,

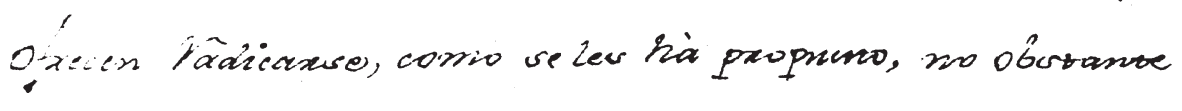

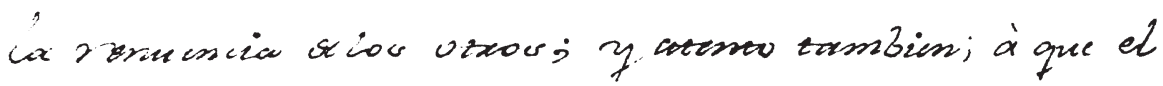
tai Cap. Cerseca blanca, con elotxo excidio num! In-

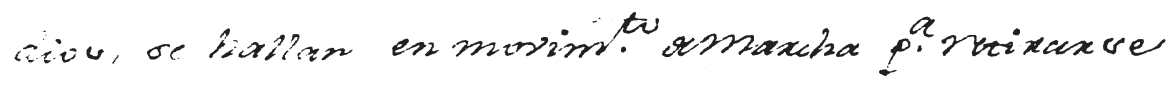

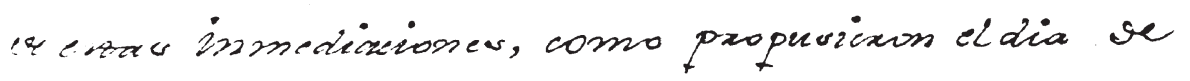

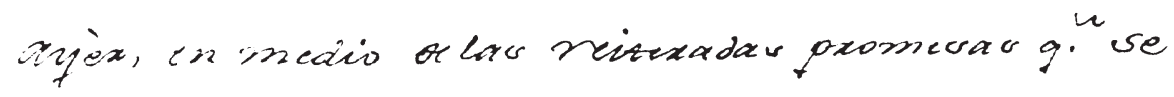

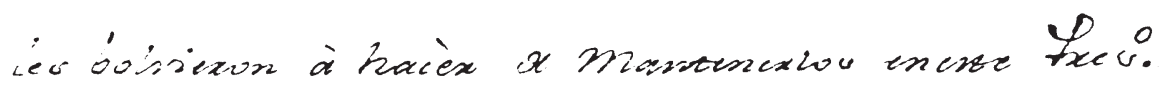

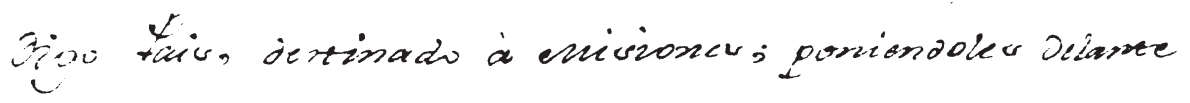
riezer quancedo mazion, $p^{a} q^{e}{ }^{e} 20$ empezasen à ev-

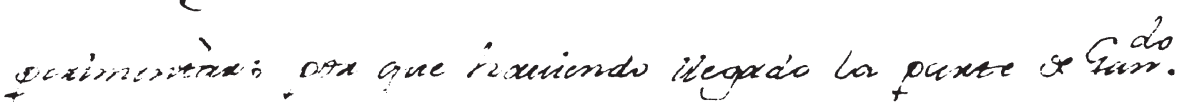
give ve mando' didcianoix corerefin, se hà podiaio

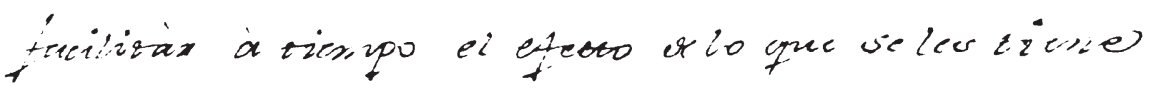

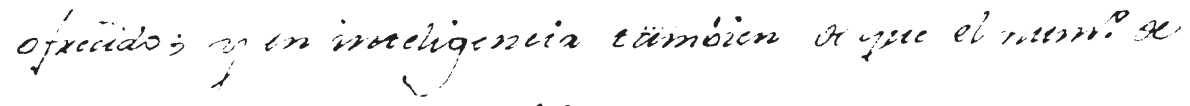
20 timdas, gi" Bisimulajam. oeles candi eniodo sue cern

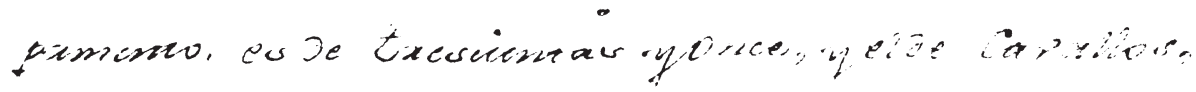


[fol. lv]

\{top margin center\} \394

\{right margin\} 162

y solicitado repetidas veces, persuaden à creer, que su

voluntàd, y la de sus inmediatos Parientes, ès distinta que

la delotro Capitàn y demàs Yndios; por que á el tiempo

que estos niegan la reduccion, y avecindamento, en Pueblos,

el Capitàn Chiquito con trescientas personas, que componen

las 32 , Tiendas de campaña que tienen junto à la suya,

ofrecen radicarse, como se les hà propuesto, no obstante

la renuncia de los otros; y atento tambien; à que el

tal Capitàn Casaca blanca, con elotro crecido numero de Yn-

dios, se hallan en movimento demarcha para retirarse

de estas inmediaciones, como propusieron el dia de

ayèr, en medio de las reiteradas promesas que se

les bolvieron à hacèr de mantenerlos en este Presidio

digo Pais, destinado à Misiones; poniendoles delante

Rezes de ganado mayòr, para que lo empezasen à es-

perimentàr; por que haviendo llegado la parte de Ganado

que se mandó adelantàr con este fin, se hà podido

facilitàr à tiempo el efecto de lo que se les tiene

ofrecido; y en inteligencia tambien de que el numero de

tiendas, que disimuladamente seles contò en todo su cam-

pamento, es de trescientas y once, y el de Cavallos, 


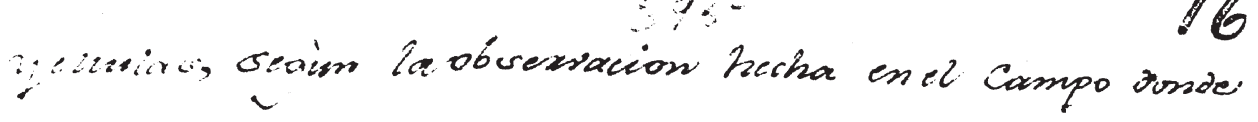
ias hiun parteado, pox low Casos, y sold. inviligeneer

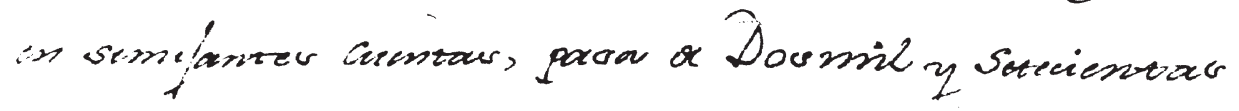
Cevizar osma, potax especien yque debe exeexse, pox

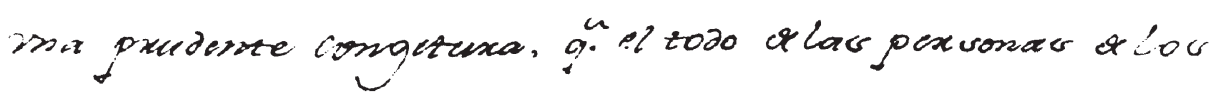

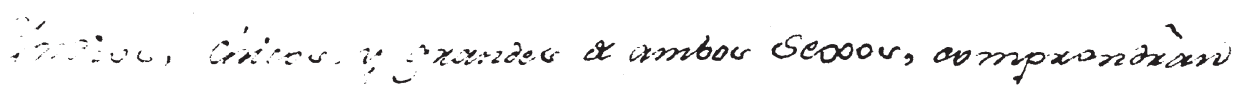

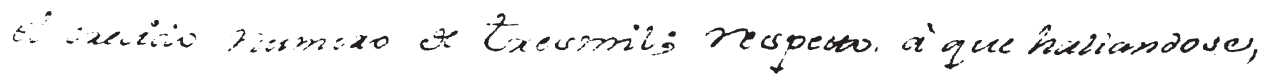

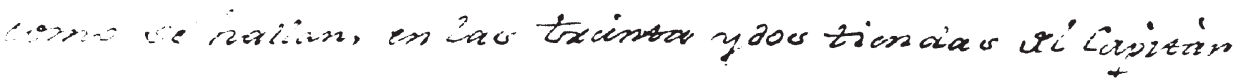

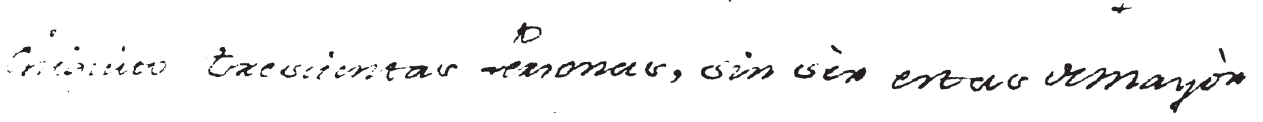

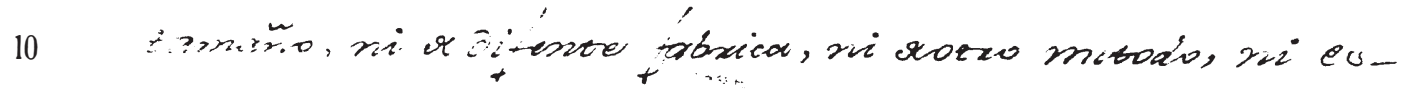

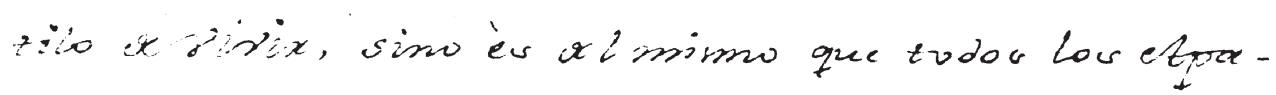

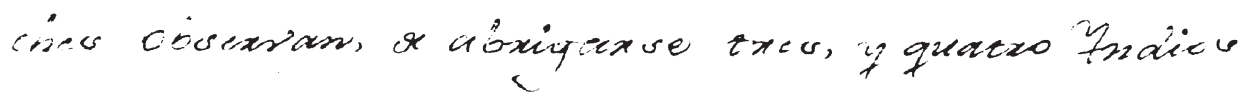

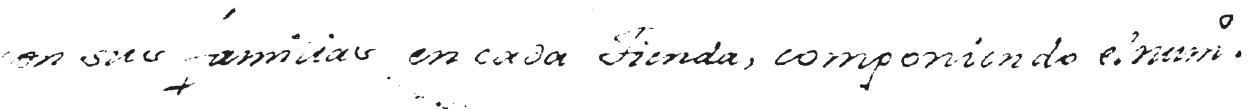

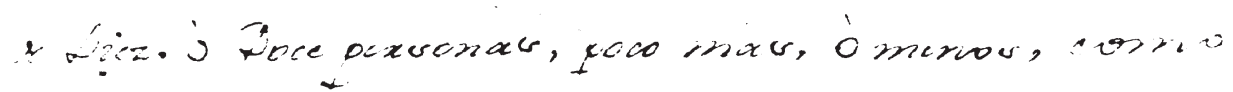

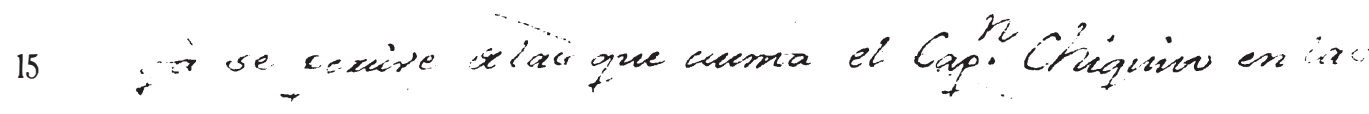

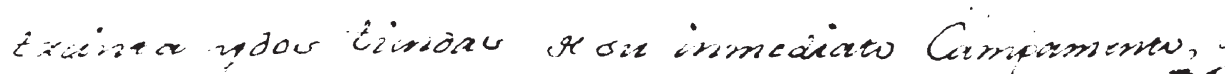

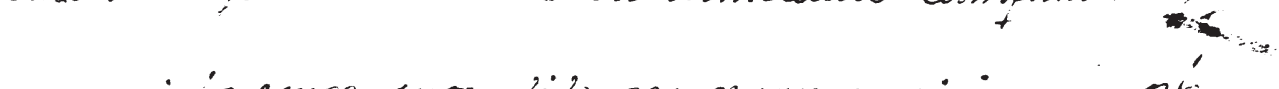

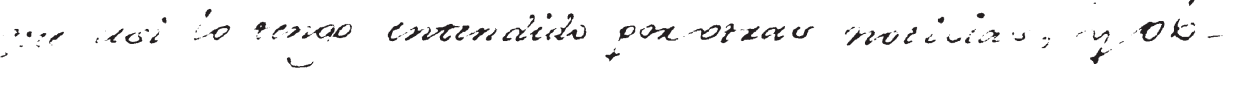

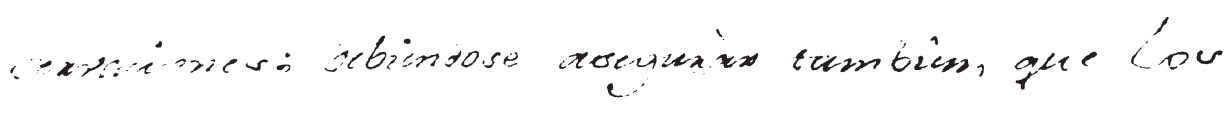

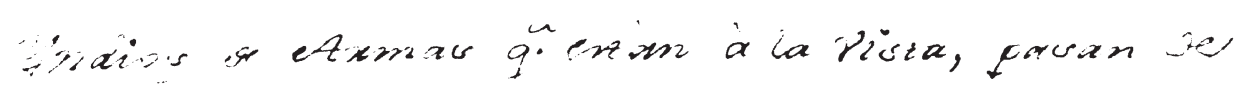

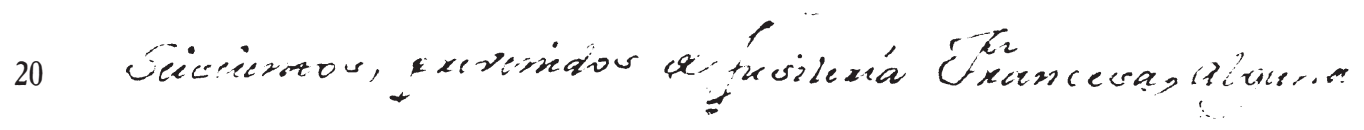

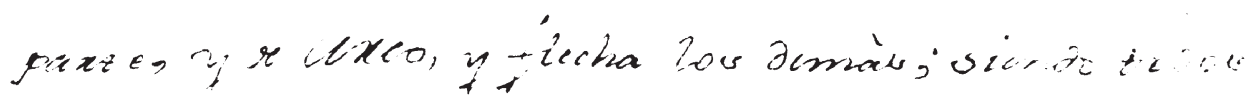


[fol. 2r]

\{top margin center\} $\backslash 395$

\{right margin\} 163

y Mulas, segùn la observacion hecha en el campo donde

las hàn pasteado, por los Cavos, y Soldados intiligentes

en semejantes cuentas, pasa de Dos mil y setecientas

Cavezas de una, y otra especie, y que debe creerse, por

una prudente congetura, que el todo de las personas de los

Yndios, chicos y grandes de ambos sexos, comprondràn

el crecido numero de tresmil; respecto à que hallandose,

como se hallan, en las treinta y dos tiendas del Capitàn

Chiquito trescientas Personas, sin sèr estas demayòr

tamaño, ni de difente [diferente] fabrica, ni deotro metodo, ni es-

tilo de vivir, sino ès del mismo que todos los Apa-

ches observan de abrigarse tres, y quatro Yndios

con sus familias en cada Tienda, componiendo elnumero

de Diez, ò Doce personas, poco mas, ò menos, como

yà se percive de las que cuenta el Capitàn Chiquito en las

treinta y dos tiendas de su inmediato Campamento, y

que asi lo tengo entendido por otras noticias, y ob-

servaciones; debiendose aseguràr tambien, que los

Yndios de Armas que estàn à la vista, pasan de

Seiscientos, prevenidos de fusilería Francesa, alguna

parte, y de arco y flecha los demàs; siendo todos 


\section{0}

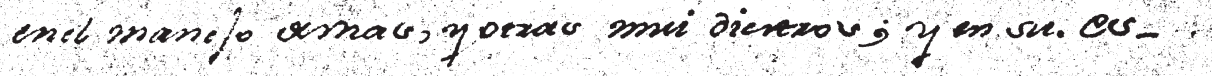

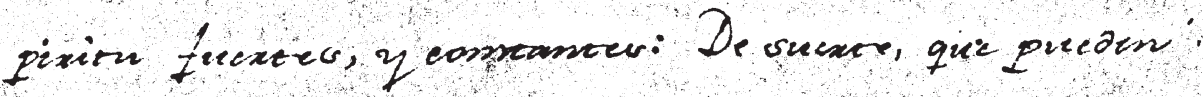

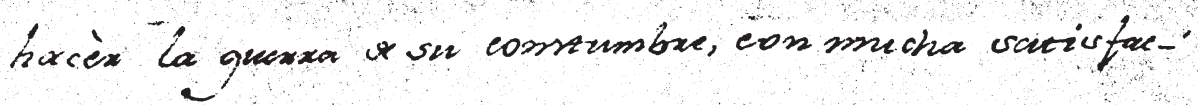

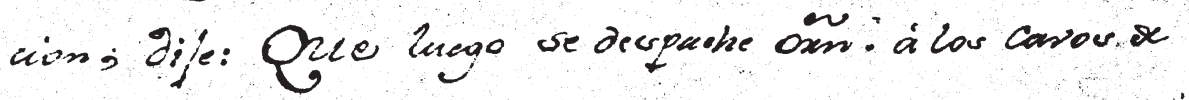

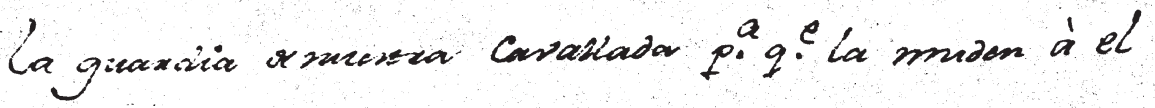

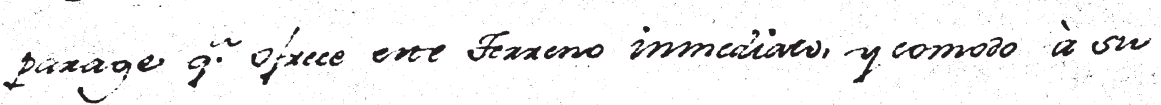

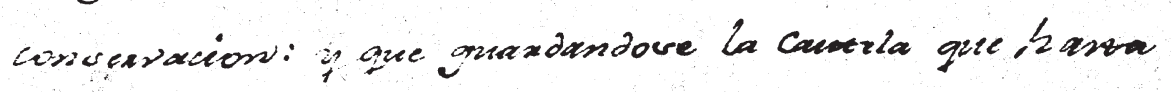

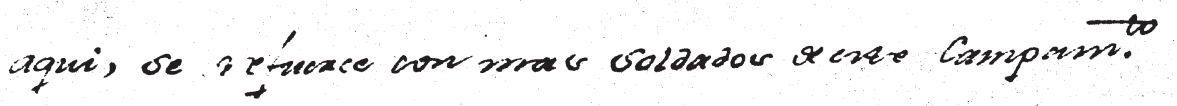

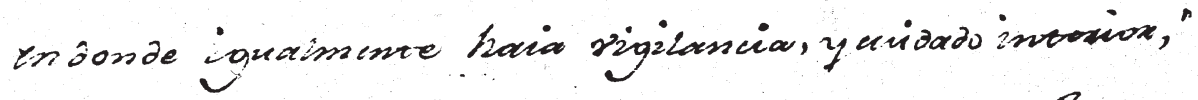

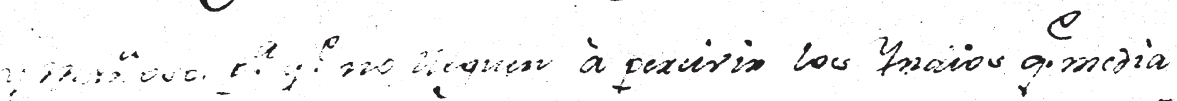

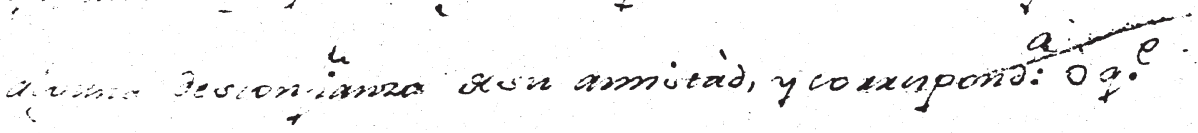

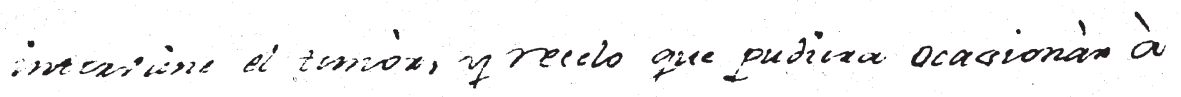

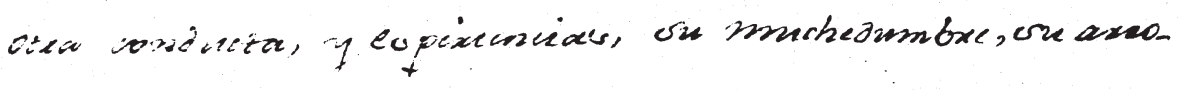

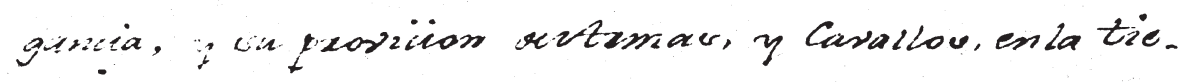

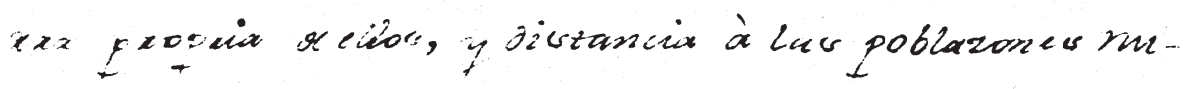

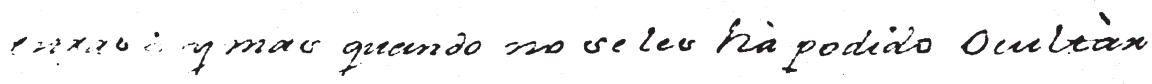

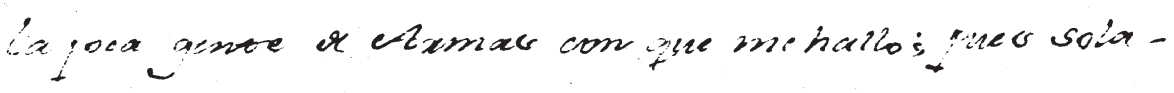

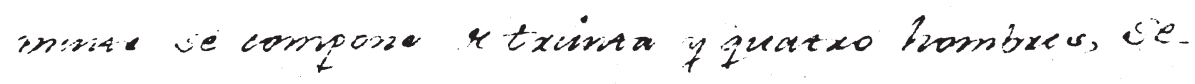

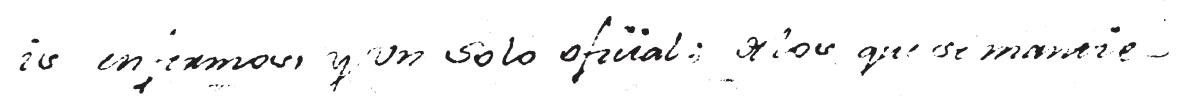

20

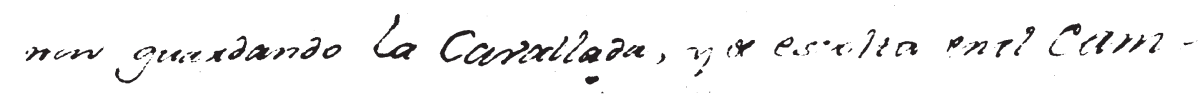

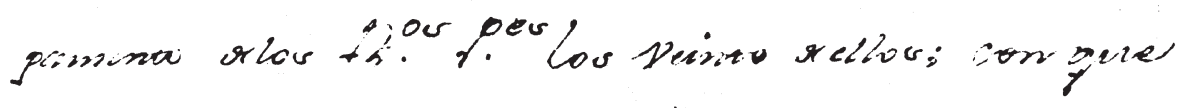




\section{[fol. $2 \mathrm{v}$ ]}

\{top margin center\} \396

\{right margin\} 164

enel manejo deunas, y otras mui diestros; y en su es-

piritu fuertes, y constantes: De suerte, que pueden

hacèr la guerra de su constumbre, con mucha satisfac-

cion; dije: Que luego se despache ordeñes à los cavos de

la guardia de nuestra cavallada para que la muden à el

parage que ofrece este Terreno inmediato, y comodo à su

conservacion: y que guardandose la cautela que hasta

aqui, se refuerce con mas soldados de este Campamento

en donde igualmente haia vigilancia, y cuidado interior,

y mañoso, para que no lleguen à percivir los Yndios que media

alguna desconfianza de su amistàd, y correspondencia: ò que

interviene el temòr, y recelo que pudiera ocasionàr à

otra conducta, y espìriencias, su muchedumbre, su arro-

gancia, y su provicion deArmas, y cavallos, en la tie-

rra propria de ellos, y distancia à las poblazones nu-

estras; y mas quando no se les hà podido ocultàr

la poca gente de Armas con que me hallo; pues sola-

mente se compone de treinta y quatro hombres, se-

is enfermos, y un solo oficial; de los que se mantie-

nen guardando la Cavallada, y de escolta enel Cam-

pamento de los Reverendos Padres los veinte de ellos; con que 
Spanish Colonial Documents Pertaining to Mission San Sabá

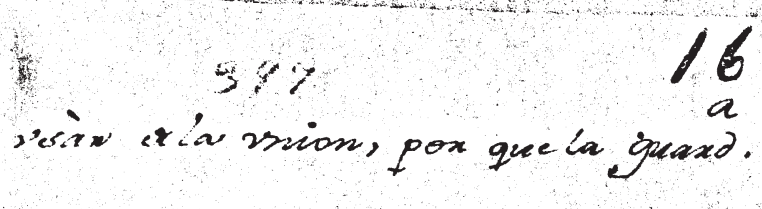

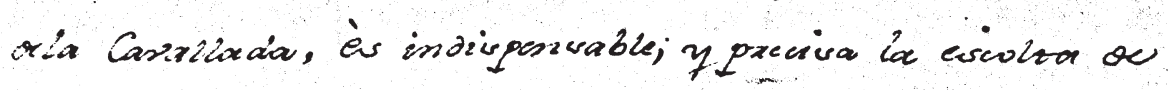

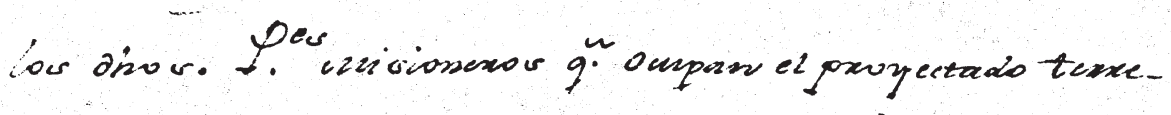

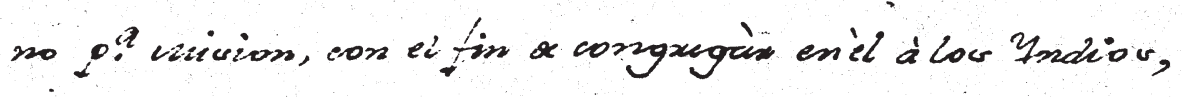

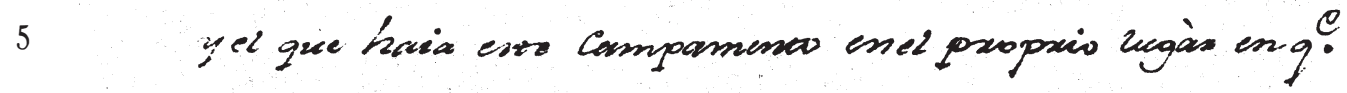
ve ialia: reswita que volo la confiaman el maxefo $x$

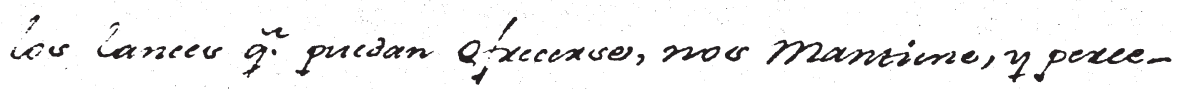

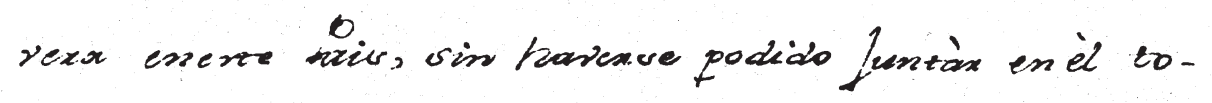

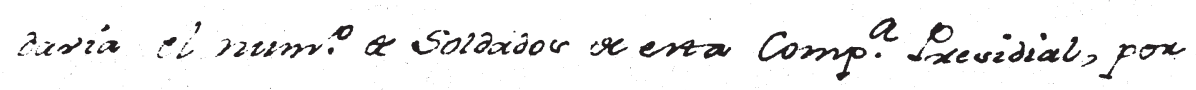

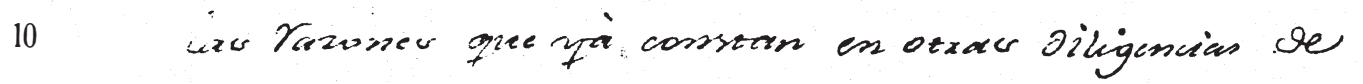
extos chutoon y que von tan pxecioar, è indispensables

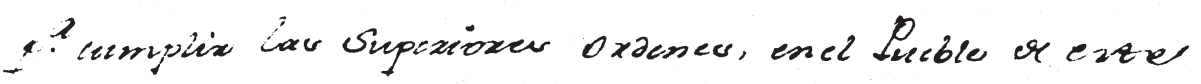

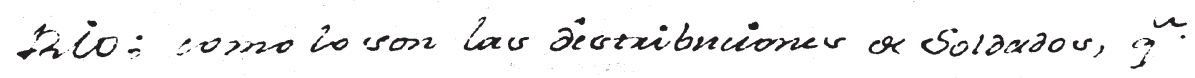
ve ricurem a eogrusains y que ve cite, y conrogue de Hrivo Capitain Chiquito, y à lov orou inmediue?

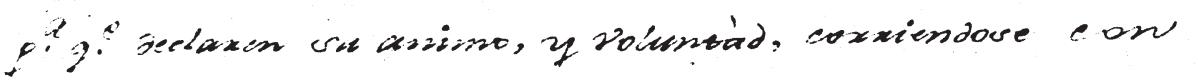

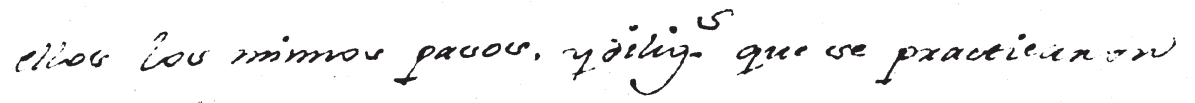

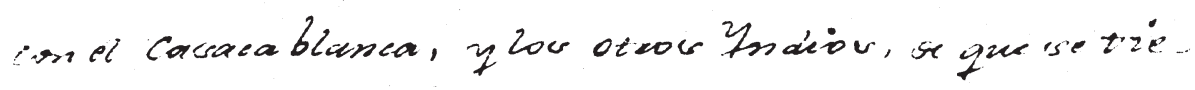

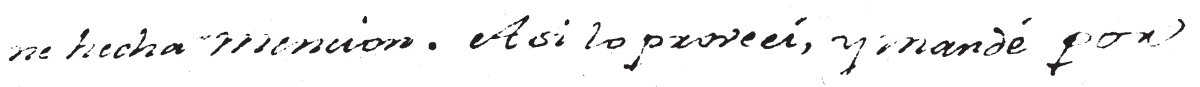

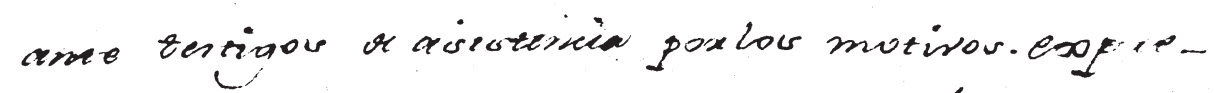

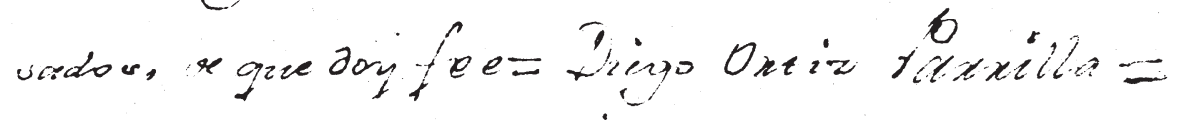


[fol. 3r]

\{top margin center\} \397

\{right margin\} 165

divididos sin podèr usàr de la union, por que la guardia de la cavallada, ès indispensable; y precisa la escolta de los dichos. Padres Misioneros que ocupan el proyectado terreno para Mision, con el fin de congregàr en èl à los Yndios, y el que haia este campamento en el proprio lugàr en que se halla; resulta que solo la confianza, $\left({ }^{\wedge} \mathrm{en}\right)$ el manejo de los lances que puedan ofrecerse, nos mantiene, y percevera en este Pais, sin haverse podido juntàr en èl todavia el numero de soldados de esta Compañia Presidial, por las razones que yà constan en otras diligencias de estos Autos, y que son tan precisas, è indispensables para cumplir las superiores ordenes, en el Pueblo de este Rio; como lo son las distribuciones de soldados, que se àcavan de expresàr; y que se cite, y convoque à el Yndio Capitàn Chiquito, y à los de su inmediacion

para que declaren su animo, y voluntàd, corriendose con ellos los mismos pasos, y diligencias que se practicaron con el Casaca blanca, y los otros Yndios, de que se tiene hecha mencion. Asi lo proveeí, y mandé por ante testigos de asistencìa por los motivos expresados, de que doy fee $=$ Diego Ortiz Parrilla $=$ 
Spanish Colonial Documents Pertaining to Mission San Sabá

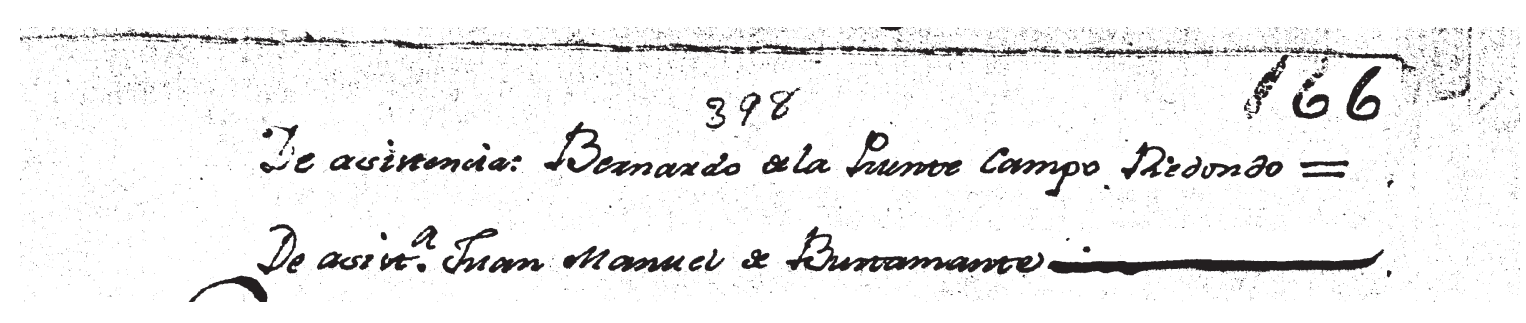


[fol. 3v]

\{top margin center\} $\backslash 398$

\{right margin\} 166

De asistencia: Bernardo de la Puente Campo Redondo = De asistencia: Juan Manuel de Bustamante 


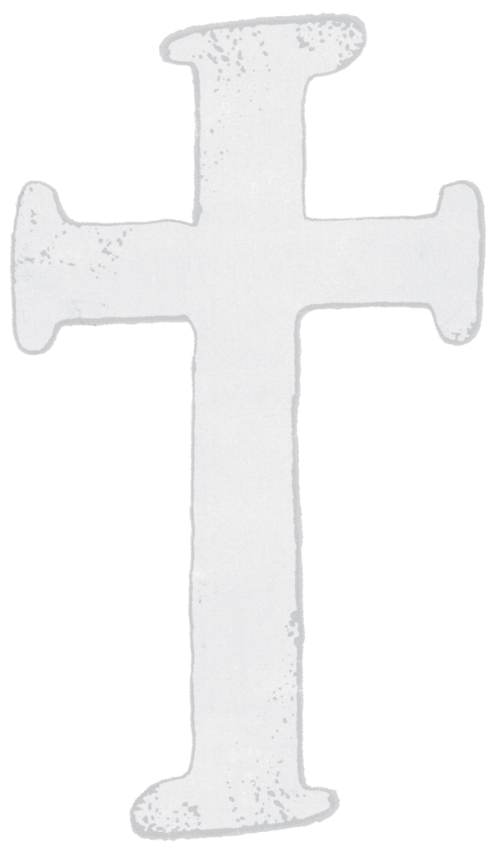




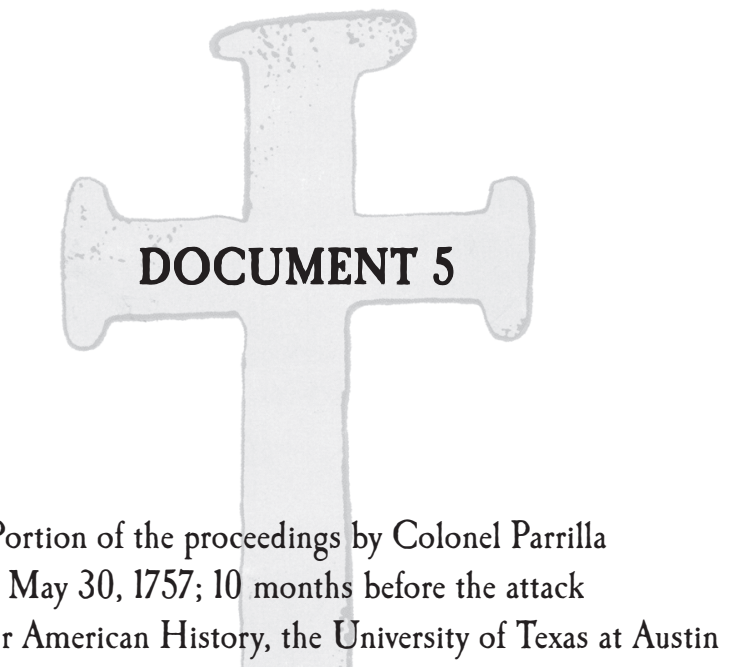


Spanish Colonial Documents Pertaining to Mission San Sabá

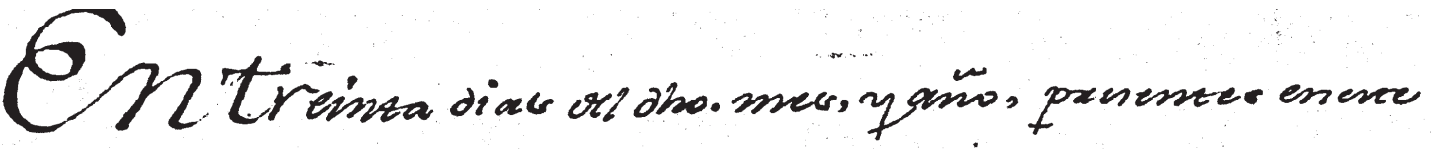

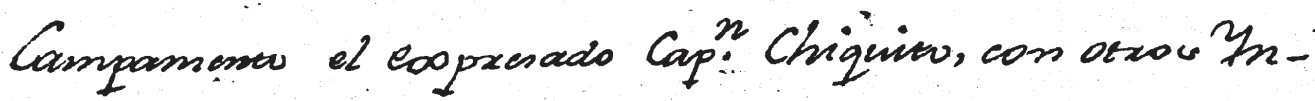

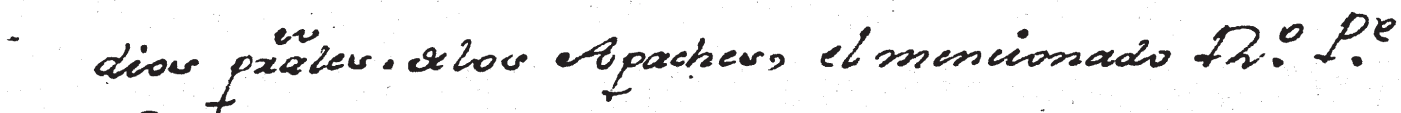

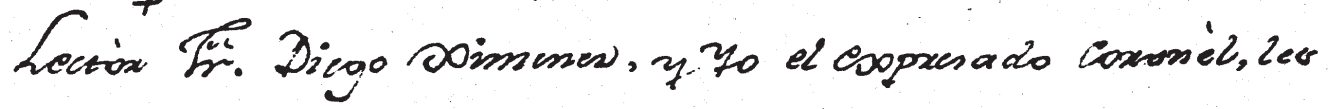
5 Liec sarien e' motivo at ser zlamada, pox medio orl In

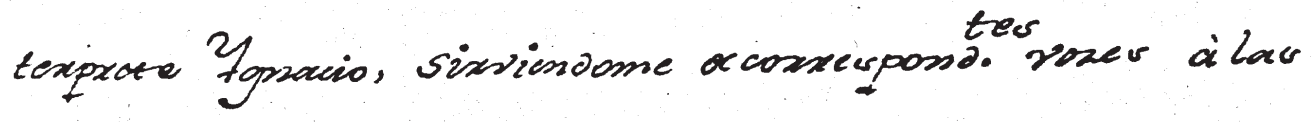
ol Torwonamt to "u ve le hizo à el Capizan Cacaca blanca, y.que comsan en la diligencia antecedenoes y haciundose

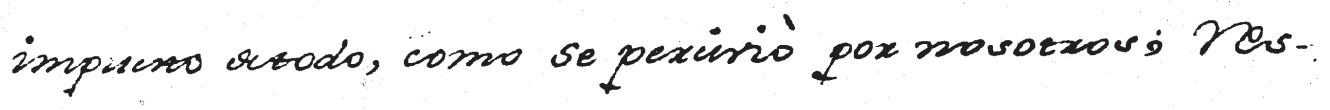

10 pondió, tamando la vos or los demàs el dho. Capizan Chiquicu:gr Que pox à hoxa no podia cumplix la n) yalabxa que teria dada ocrolocunere con las tresciontas. 9) Hexvonar promocidar en incivions pox que el Cacouca

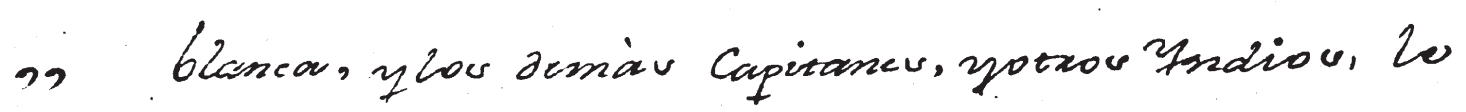
15 - hovian pediulo 2loxando, que no Les desampaxase 1) enla ocavion que tenian determinado huex carn1) Puño contra Lor Indios Cumoncher, y oalix à bas-

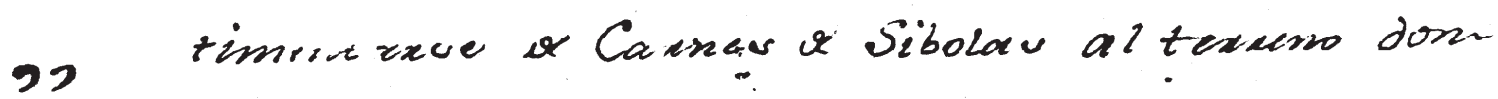
nn de las hai; "y que it morido ut caximo ge à todos

68 
[fol. 1r]

\{top margin center\} \398

\{right margin\} 166

EN TReinta dias del dicho mes, y año, presentes en este

Campamento el expresado Capitàn Chiquito, con otros Yn-

dios principales de los Apaches, el mencionado Reverendo Padre

Lectòr Fray Diego Ximenez, y yo el expresado coronèl, les

hice savèr el motivo de su llamada, por medio del Yn-

terprete Ygnacio, sirviendome de correspondentes vozes à las

del razonamento que se le hizo à el Capitan Casaca blanca,

y que constan en la diligencia antecedente; y haviendose

impuesto detodo, como se perciviò por nosotros; res-

pondiò, tomando la voz de los demàs el dicho Capitan

Chiquito: "QUE por à hora no podia cumplir la

"palabra que tenia dada decolocarse con las trescientas

"Personas prometidas en Mision, por que el Casaca

"blanca, y los demàs Capitanes, yotros Yndios, le

"havian pedido llorando, que no les desamparase

"en la ocasion que tenian determinado hacer cam-

"paña contra los Yndios Cumanches, y salir à bas-

“timentarse de Carnes de Sibolas al terreno don-

"de las hai; y que èl movido del cariño que à todos 
Spanish Colonial Documents Pertaining to Mission San Sabá

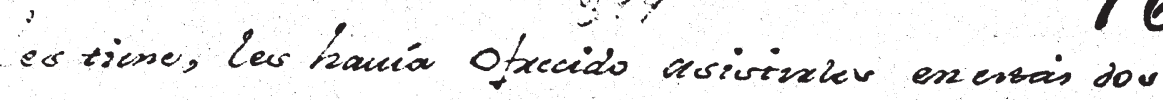

$1-$

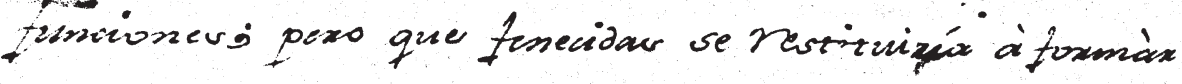

exisionew, conzo tione dicho: aseguxando ersa nueva

pxomesa con michas espaceionev. y, un melanco-

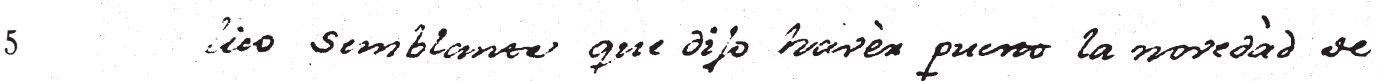
0

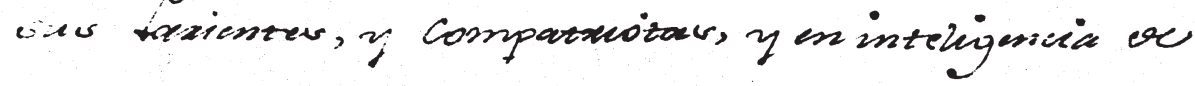

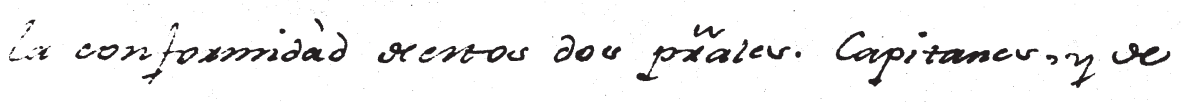

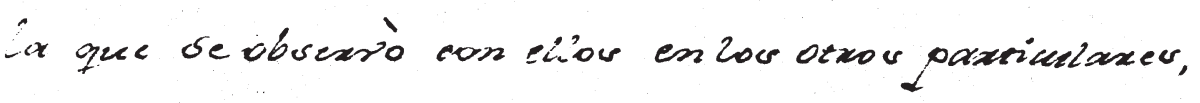

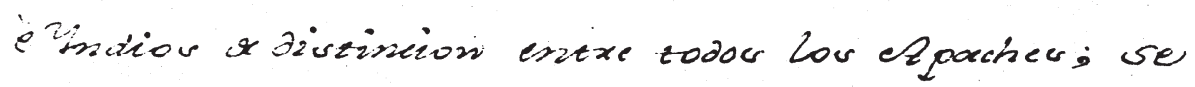

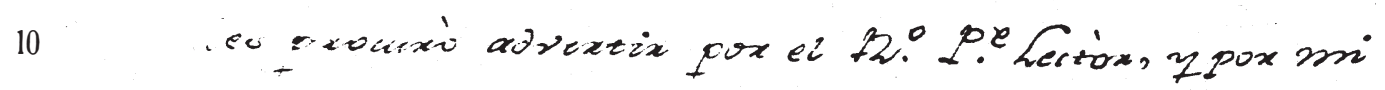

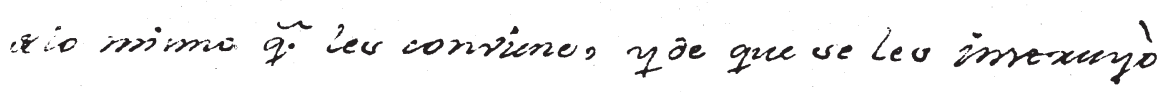

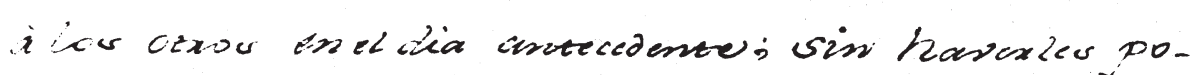

ciicio fexcinatix à ozxa cova apxomo, srav gue à

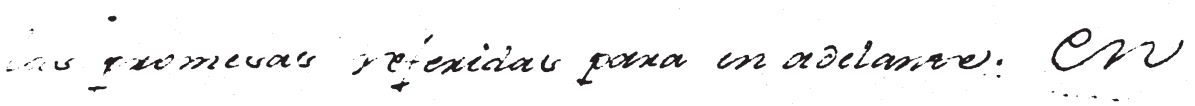

15

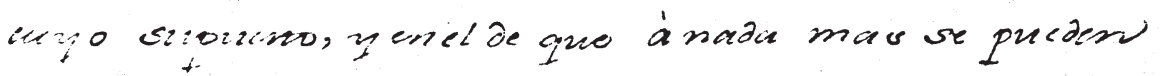

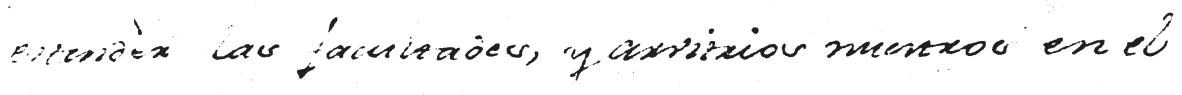

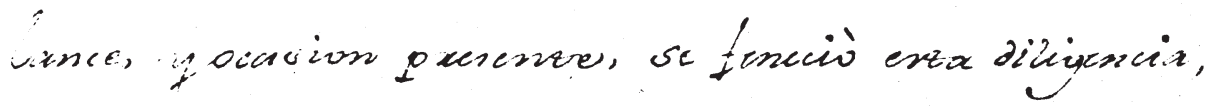

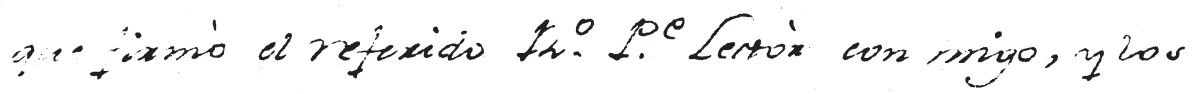

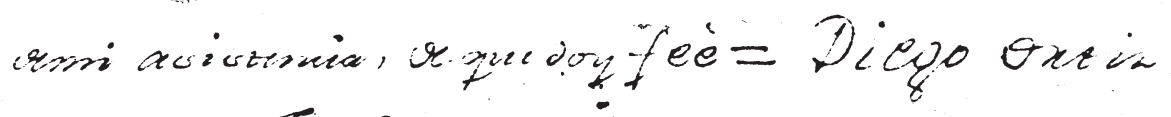

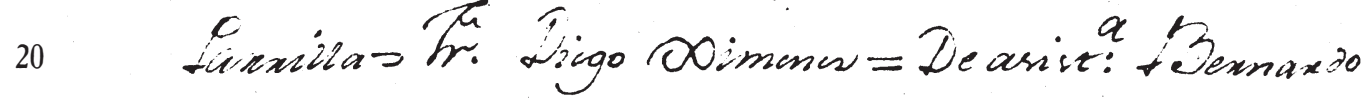


[fol. lv]

\{top margin center\} \399

\{right margin\} 167

les tiene, les havía ofrecido asistirles en estás dos

Funciones; pero que fenecidas se restituiría à formàr

Misiones, como tiene dicho; asegurando èsta nueva

promesa con muchas espresiones, y un melanco-

lico semblante que dijo havèr puesto la novedàd de

sus Parientes, y compatriotas, y en inteligencia de

la conformidàd de estos dos principales Capitanes, y de

la que se observò con ellos en los otros particulares,

è Yndios de distincion entre todos los Apaches; se

les procurò advertir por el Reverendo Padre Lectòr, y por mi

de lo mismo que les conviene, y de que se les instruyò

à los otros en el dia antecedente; sin haverles po-

dido persuadir à otra cosa depronto, mas que à

las promesas referidas para en adelante. EN

cuyo supuesto, y en elde que à nada mas se pueden

estendèr las facultades, y arvitrios nuestros en el

lance, y ocasion presente, se feneciò esta diligencia,

que firmò el referido Reverendo Padre Lectòr con migo, y los

demi asistencia, de que doy feè = Diego Ortiz

Parrilla $=$ Fray Diego Ximenez = De asistencia: Bernardo 
Spanish Colonial Documents Pertaining to Mission San Sabá

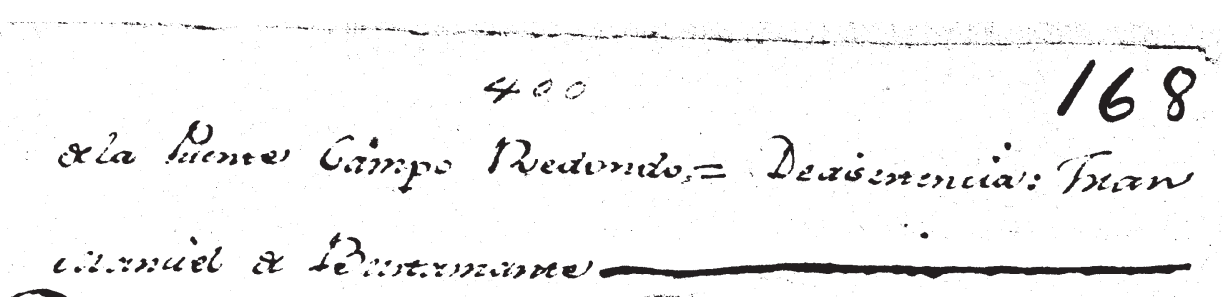


[fol. 2r]

\{top margin center $\} \backslash 400$

\{right margin\} 168

de la Puente Campo Redondo,= De asistencia: Juan

Manuèl de Bustamante 


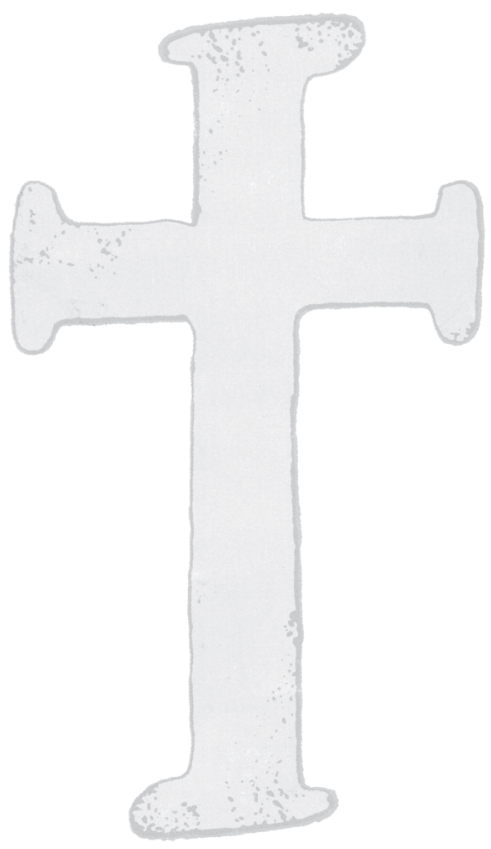




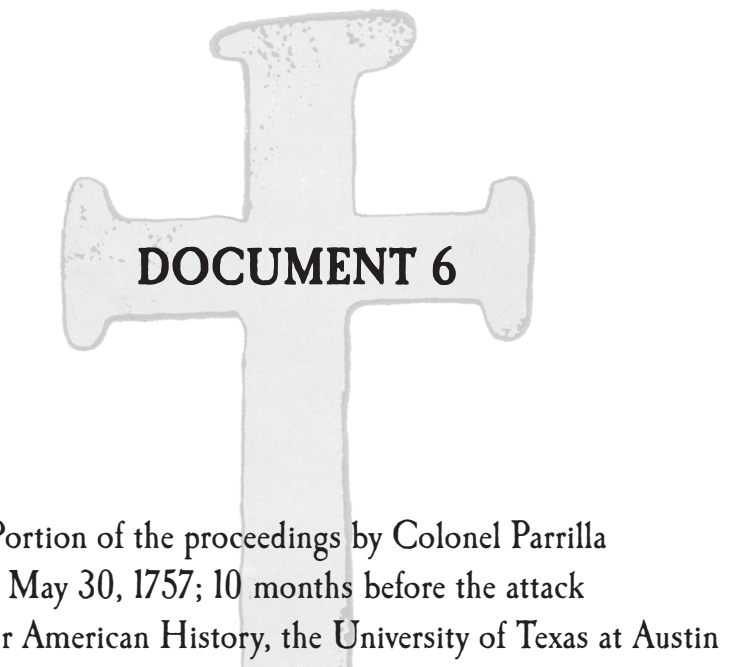


Spanish Colonial Documents Pertaining to Mission San Sabá

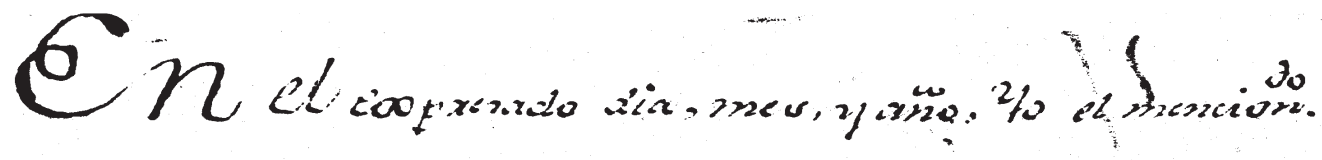

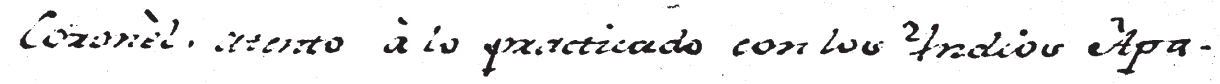

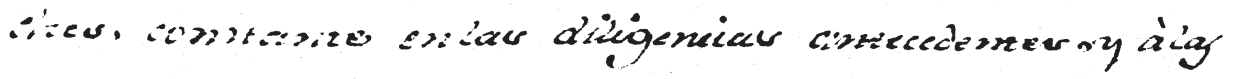

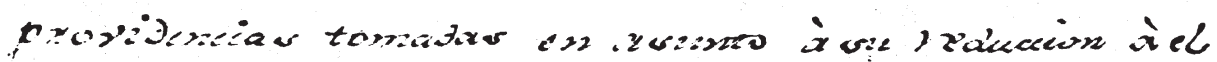

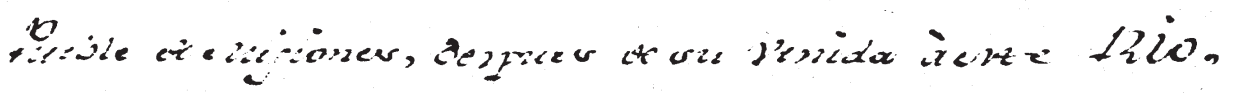

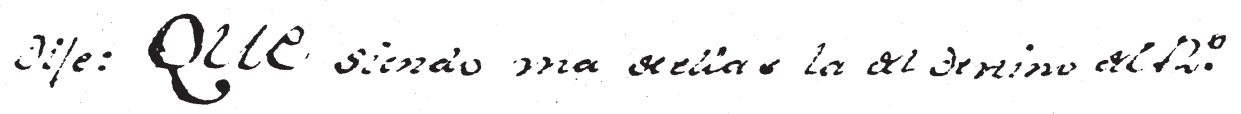

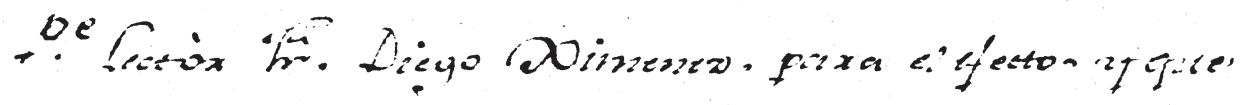

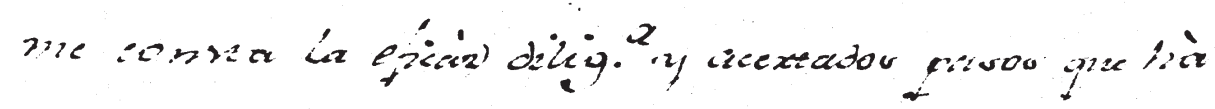

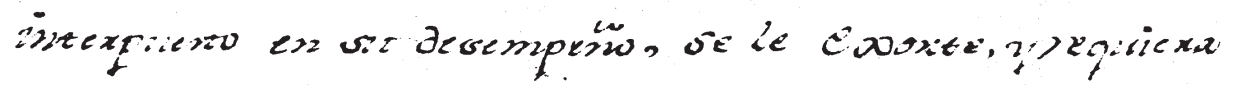

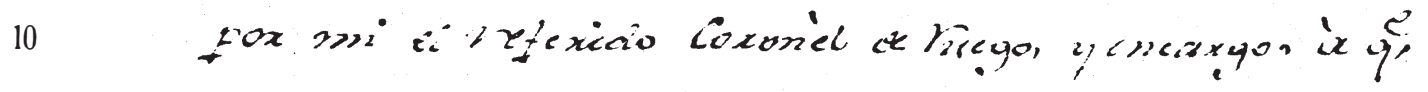

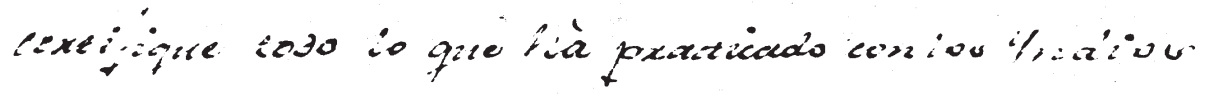

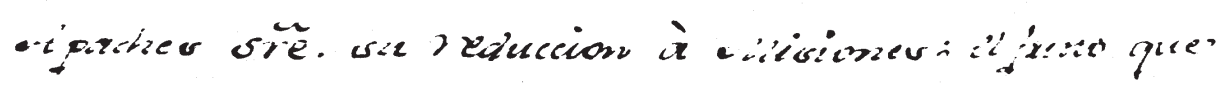

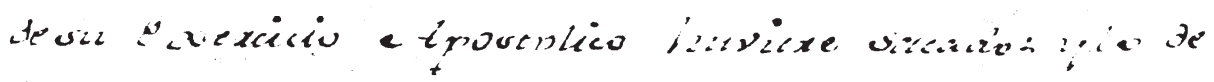

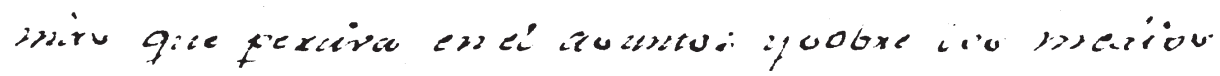

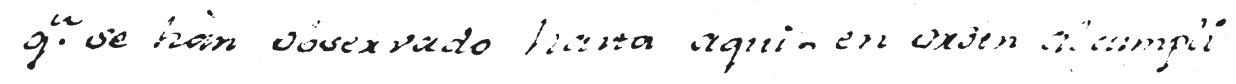

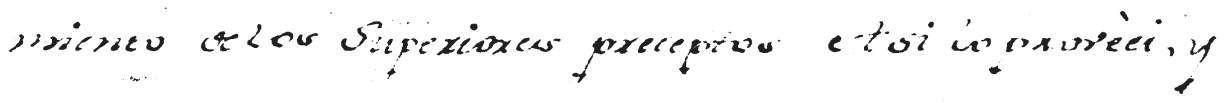

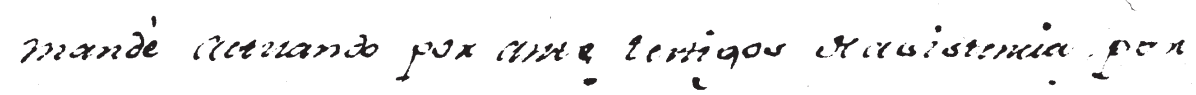

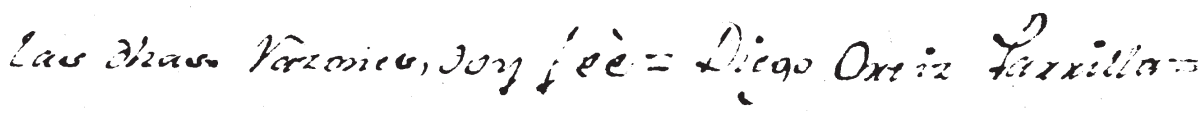


[fol. lr]

\{top margin center $\} \backslash 400$

\{right margin right 168

EN El expresado dia, mes, y año, Yo el mencionado

Coronèl atento à lo practicado con los Yndios Apa-

ches, constante en las diligencias antecedentes, y à las

providencias tomadas en asunto à su reduccion à el

Pueblo de Misiones, despues de su venida à este Rio,

dije: QUE siendo una de ellas la del destino del Reverendo

Padre Lectòr Fray Diego Ximenez, para el efecto, y que

me consta la eficàz diligencia y acertados pasos que hà

interpuesto en su desempeño, se le exorte, y requiera

por mi el referido coronèl de ruego, y encargo, à que

certifique todo lo que hà practicado con los Yndios

Apaches sobre su reduccion à Misiones; el fruto que

de su exercicio Apostolico huviere sacado; y lo de

màs que perciva en el asunto; y sobre los medios

que se hàn observado hasta aqui, en orden al cumpli

miento de los Superiores preceptos Asi lo provèei, y

mandè actuando por ante testigos de asistencia, por

las dichas razones, doy feè = Diego Ortiz Parrilla= 
Spanish Colonial Documents Pertaining to Mission San Sabá

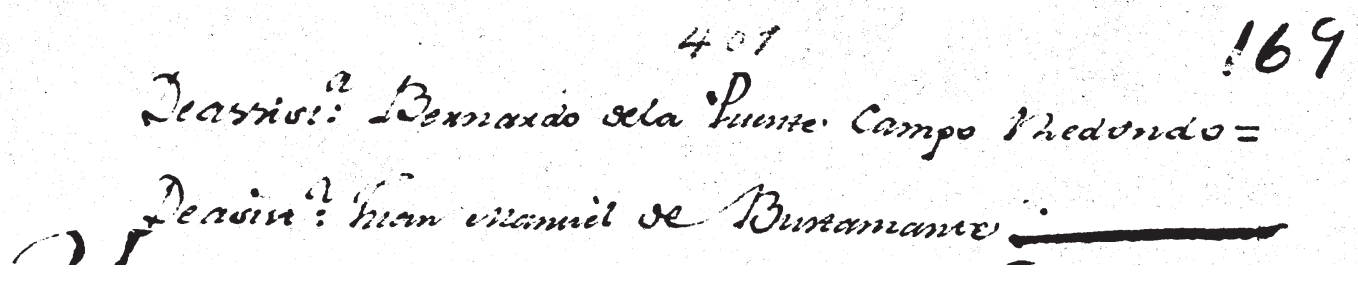


[fol. lv]

\{top margin center\} $\backslash 401$

\{right margin\} 169

De asistencia: Bernardo dela Puente Campo Redondo = De asistencia: Juan Manuèl de Bustamante 


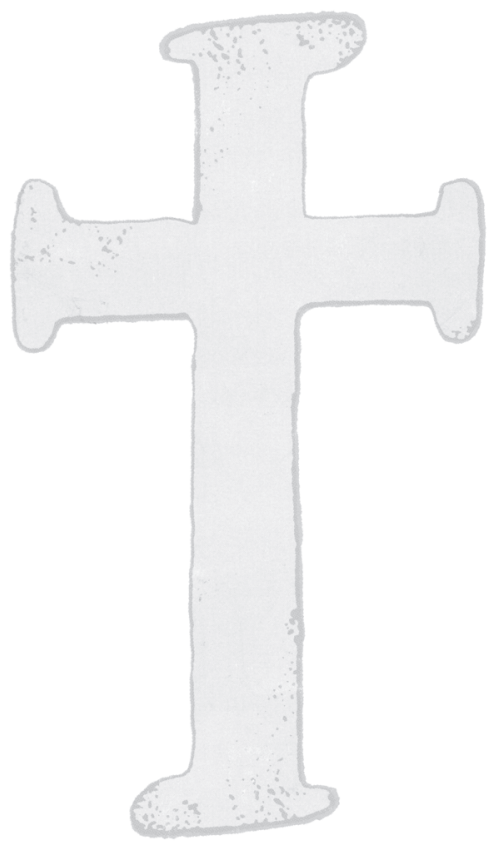




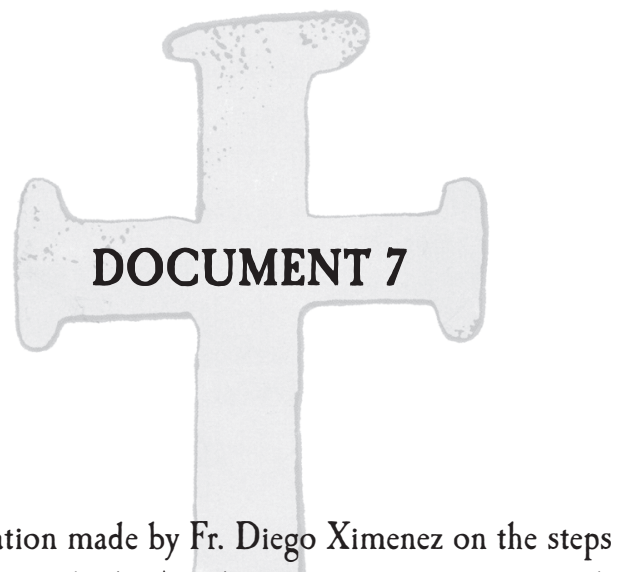

Certification made by Fr. Diego Ximenez on the steps taken to persuade the Apache to settle in a mission pueblo June 4, 1757; 10 months before the attack

Center for American History, the University of Texas at Austin 
Spanish Colonial Documents Pertaining to Mission San Sabá

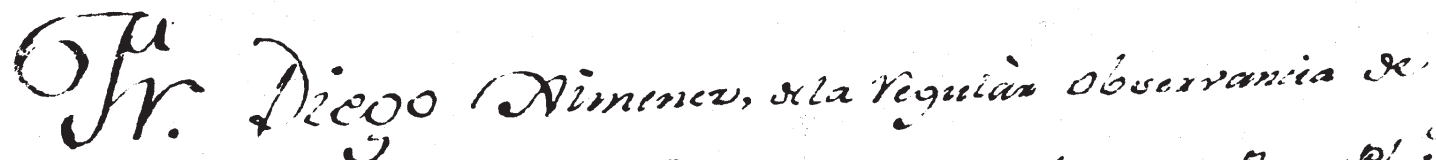

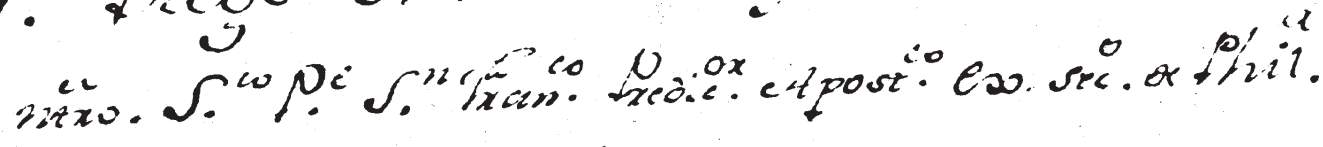

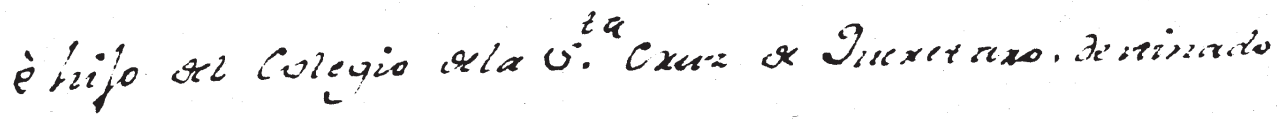

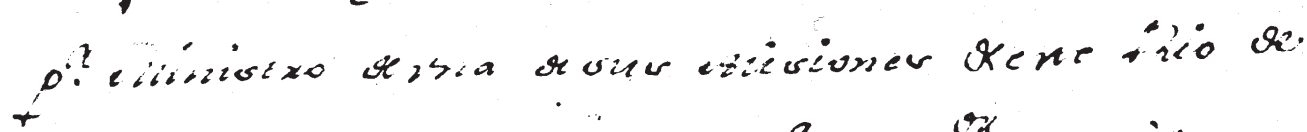

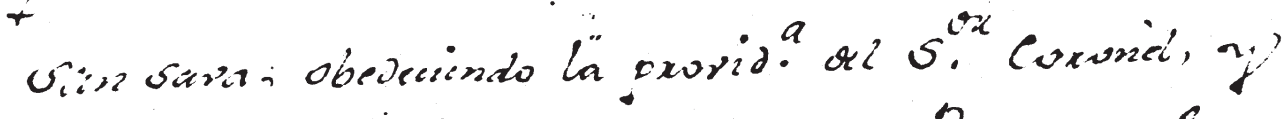

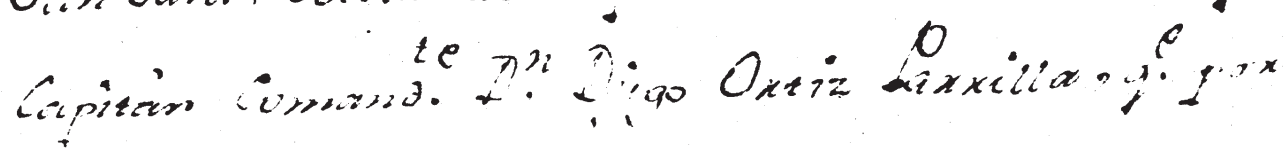

82 
[fol. 1r]

\{top margin center\} $\backslash 401$

\{right top margin\} 169

FRAY DIEGO Ximenez de la regulàr observancia de nuestro Santo Padre San Francisco Predicador Apostolico ex. secretario[???] de Philosophia è hijo del Colegio dela Santa Cruz de Queretaro, destinado para Ministro de una de sus Misiones de este Rio de San Sava, obedeciendo la providencia del Señor Coronèl, y Capitàn Comandante Don Diego Ortiz Parrilla, que por 


\section{0}

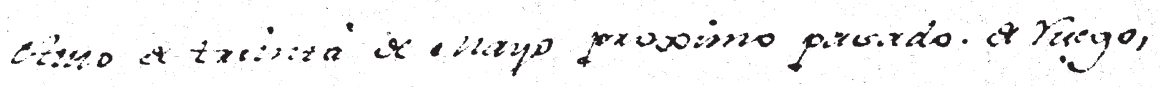

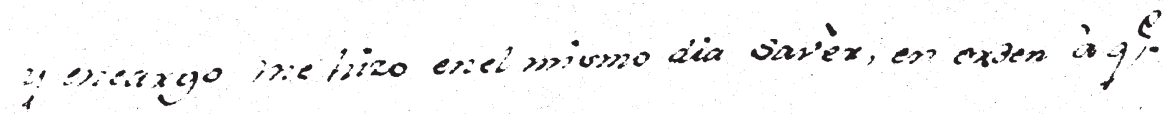

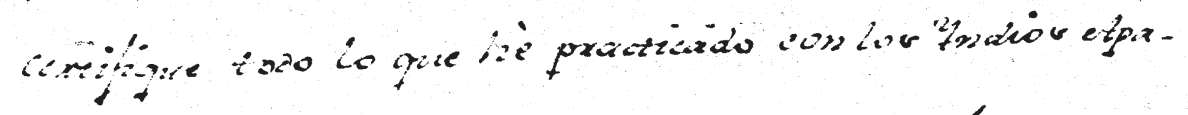

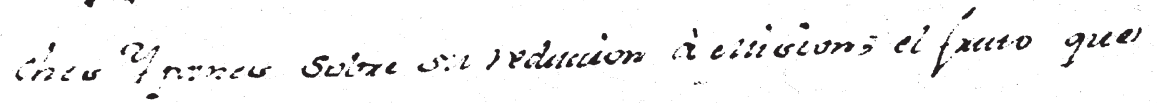

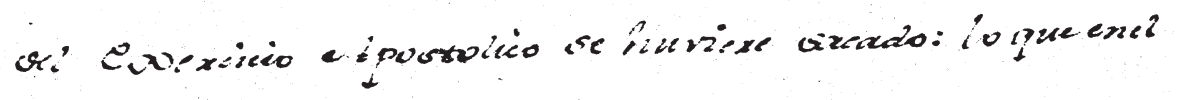

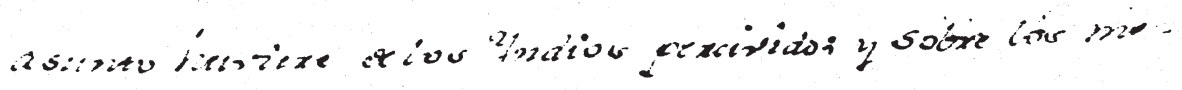

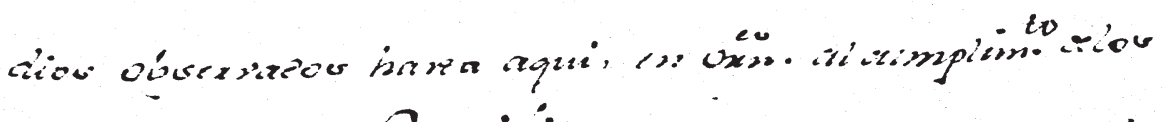

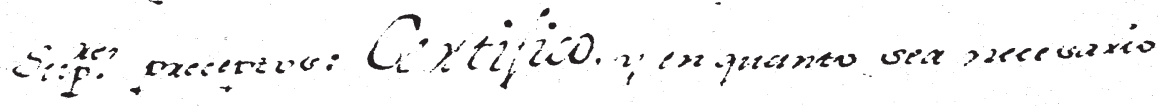

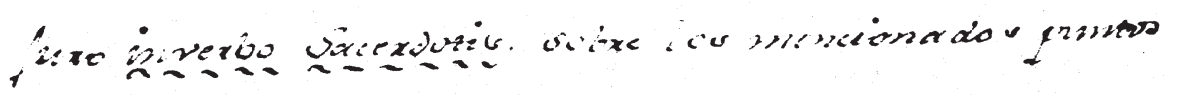

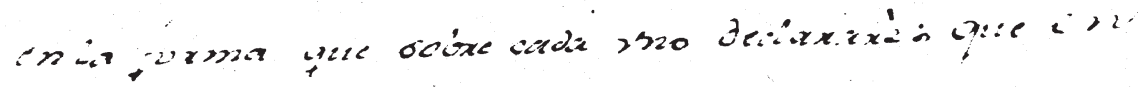

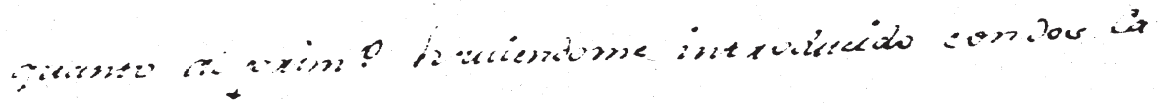

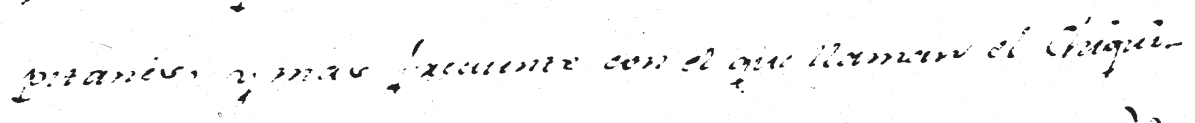

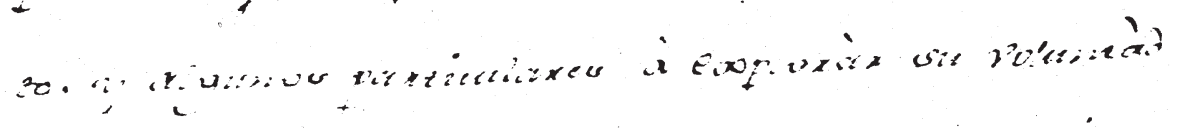

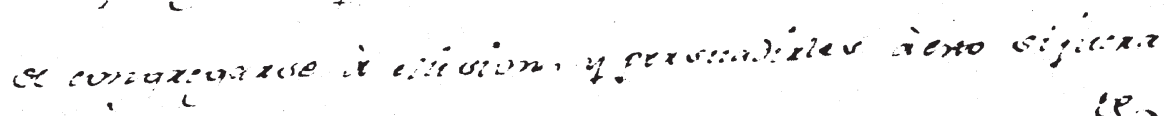

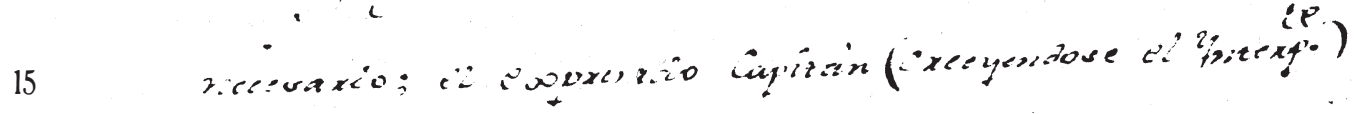

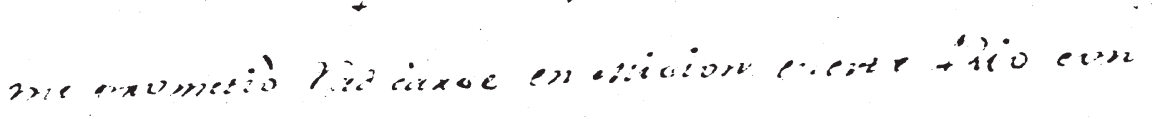

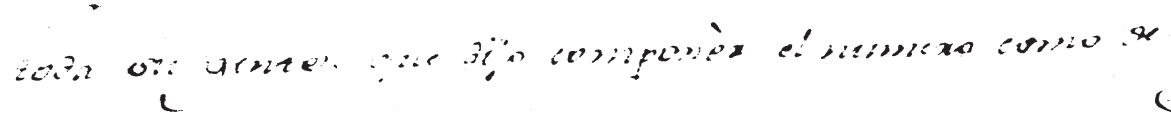

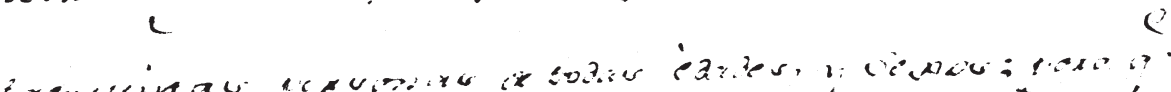

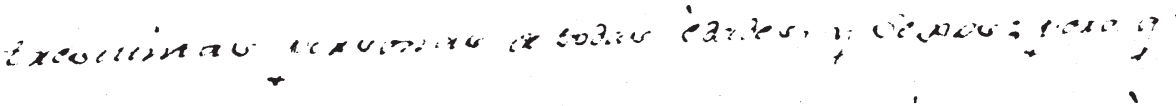

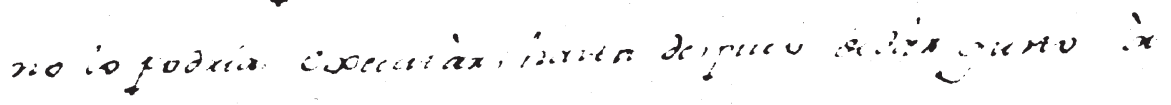

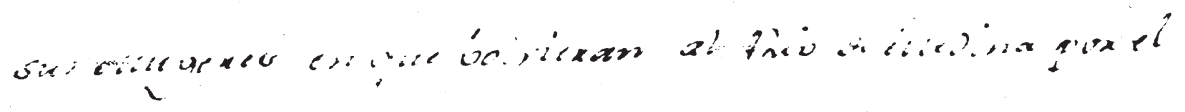

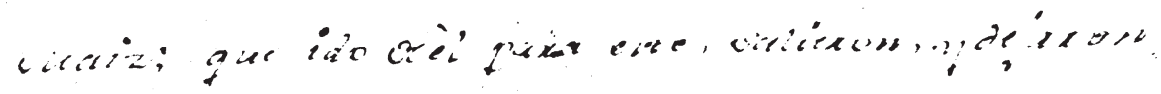

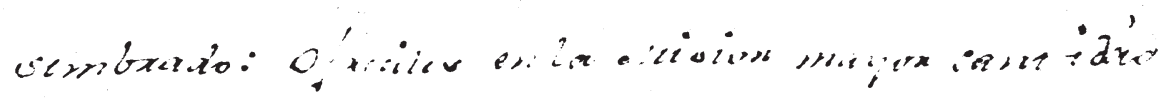


[fol. lv]

\{top margin center $\} \backslash 402$

\{top margin right 170

Auto de treinta de Mayo proximo pasado, de ruego, y encargo me hizo en el mismo dia savèr, en orden à que certifique todo lo que hè practicado con los Yndios Apaches Ypanes sobre su reduccion à Mision; el fruto que del exercicio Apostolico se huviere sacado: lo que en el asunto huviere de los Yndios percivido; y sobre los medios observados hasta aqui, en orden al cumplimento de los Superiores preceptos: CERTIFICO, y en quanto sea necesario juro inverbo sacerdotis sobre los mencionados puntos en la forma que sobre cada una declararè; que en quanto al primero haviendome introducido con dos ca pitanes, y mas frecuente con el que llaman el Chiquito, y algunos particulares à exploràr su voluntàd de congregarse à Mision, y persuadirles à esto si fuera necesario; el expresado Capitàn (creeyendose el Ynterprete) me prometiò radicarse en Mision en este Rio con toda su gente, que dijo componèr el numero como de trescientas personas de todas èdades, y sexos; pero que no lo podría executàr, hasta despues de dár gusto à sus mugeres en que bolvieran al Rio de Medina por el Maiz; que ido de èl para este, salieron, y dejaron sembrado: ofreciles en la Mision mayor cantidád 
Spanish Colonial Documents Pertaining to Mission San Sabá

\section{3}

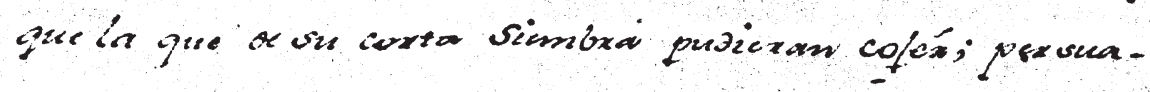

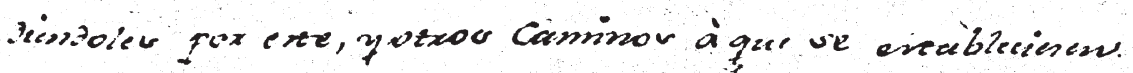

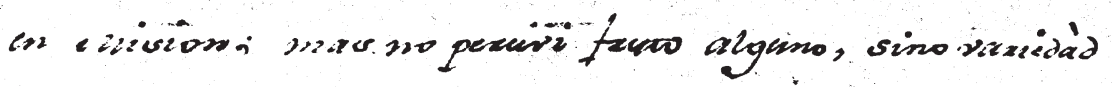

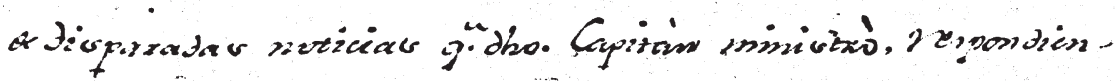

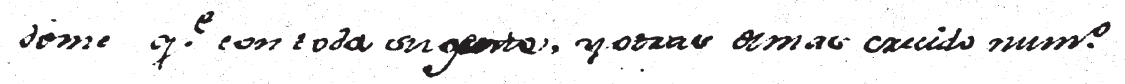

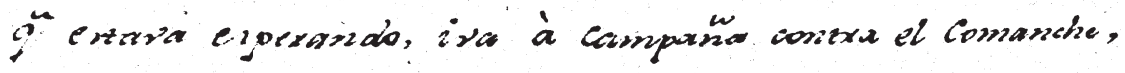

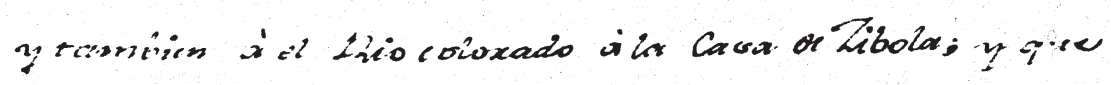

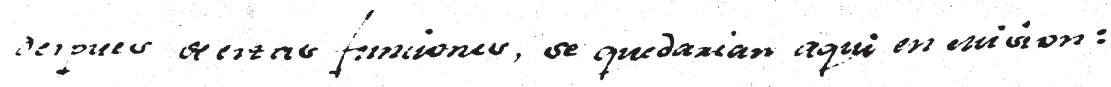

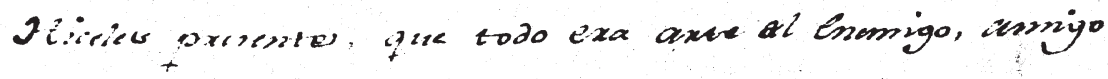

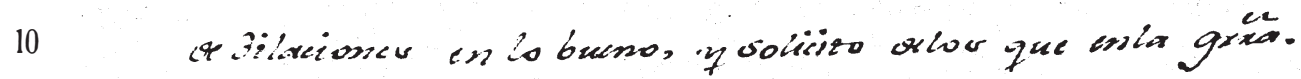

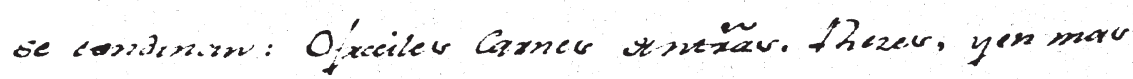

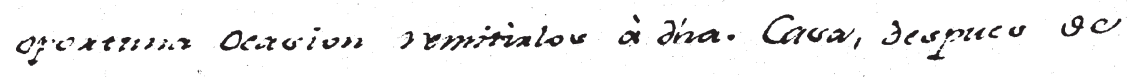

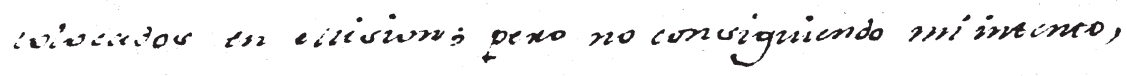

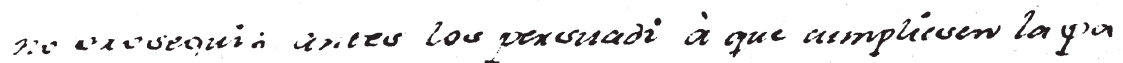

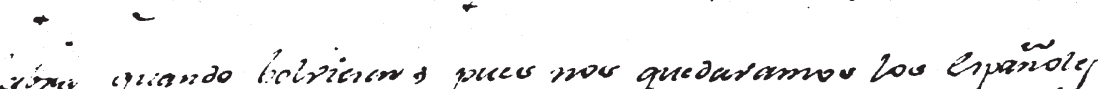

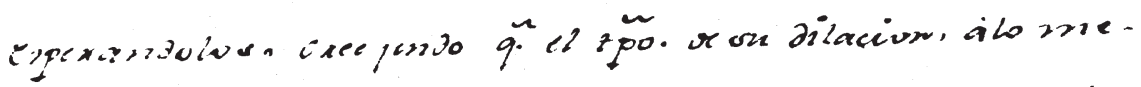

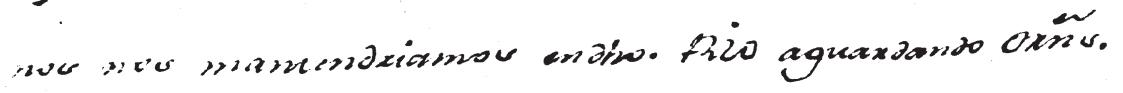

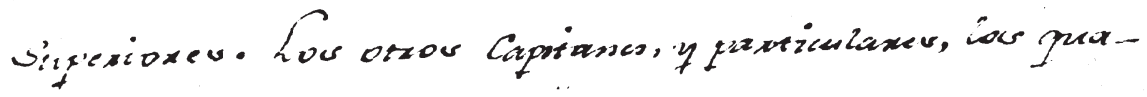

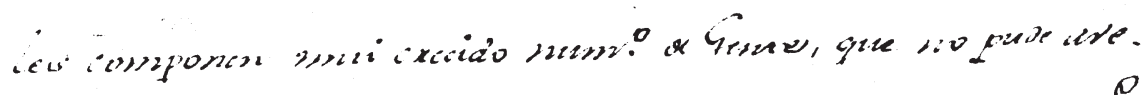

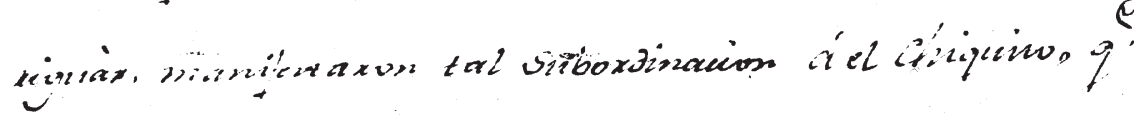

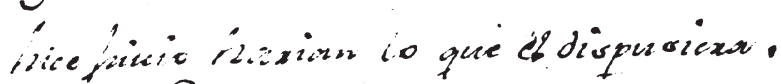
Cilixe enow tarcios
encontié zxev dipoutcizar. 
[fol. 2r]

\{top margin center\} $\backslash 403$

\{right margin\} 171

que la que de su corta siembra pudieran cojér; persuadiendoles por este, y otros caminos à que estableciesen en Mision; mas no percivi fruto alguno, sino variedàd de disparadas noticias que dicho Capitàn ministrò, respondiendome que con toda su gente, y otras demas crecido numero que estava esperando, iva à campaña contra el Comanche, y tambien à el Rio colorado à la Casa de Zibola; y que despues de estas funciones, se quedarian aqui en Mision: Hiceles presente, que todo era arte del enemigo, amigo de dilaciones en lo bueno, y solicito delos que en la guerra se condenan; Ofreciles carnes de nuestras Rezes, y en mas oportuna ocasion remitirlos à dicha Casa, despues de colocados en Mision; pero no consiguiendo mi intento, no prosegui; antes los persuadi à que cumpliesen la pa labra quando bolviesen; pues nos quedavamos los españoles esperandolos, cree yendo que el tiempo de su dilacion, à lo menos nos mantendriamos en dicho Rio aguardando ordenes Superiores. Los otros Capitanes, y particulares, los quales componen mui crecido numero de Gente, que no pude averiguàr manifestaron tal subordinacion á el Chiquito, que hice juicio harian lo que el dispusiera.

II ENTRE estos Yndios encontrè tres Apostatas, 


$$
40 \%
$$

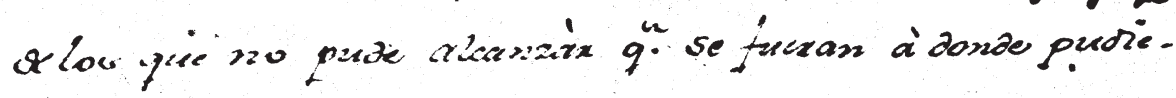

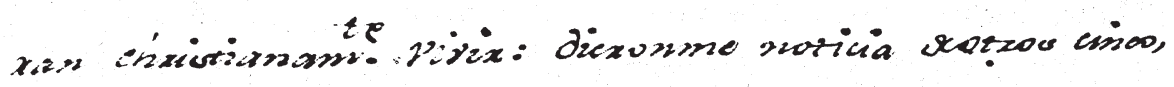

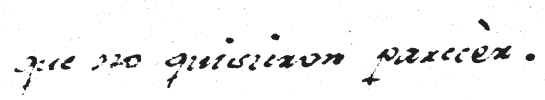

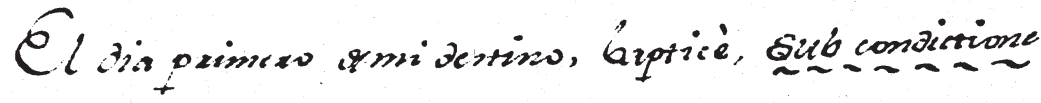
in anicsto moxtis, à un Indio, y ma tudia or mediema

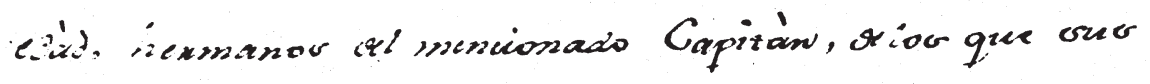

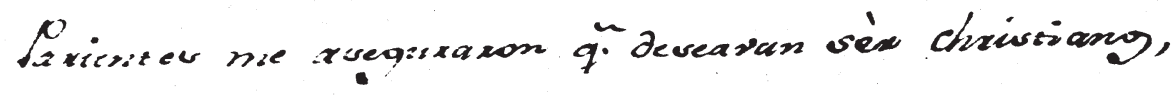

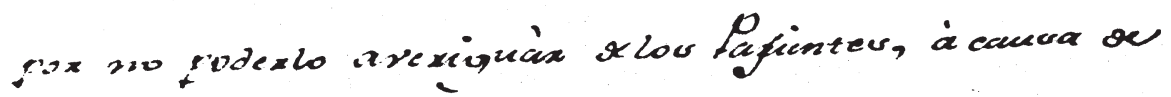

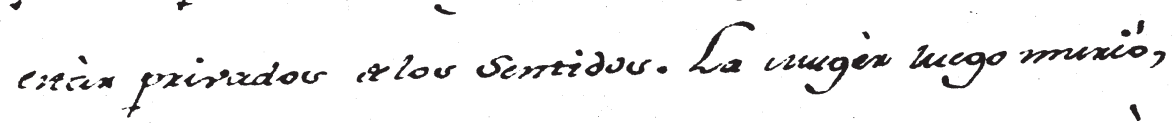

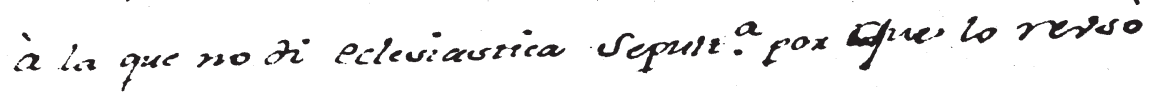
vin y'uxintela: sobxe is que no imeis mucho, espexan. 3o pxactiedito en inefón orcesion. Del hambere no vitpe inzal, pox que los te unfam." se gexixamon

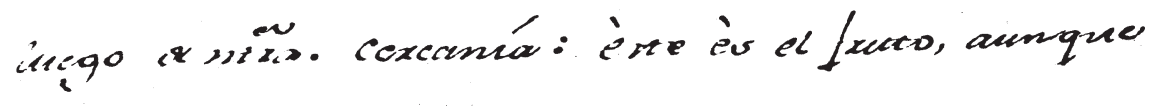
T.t:in inciexeo ormi exoriecio. 672 uxden à certificér lo que he pexierido con nầ. neyocio nu me is posible. pox la concrariciad an Lar

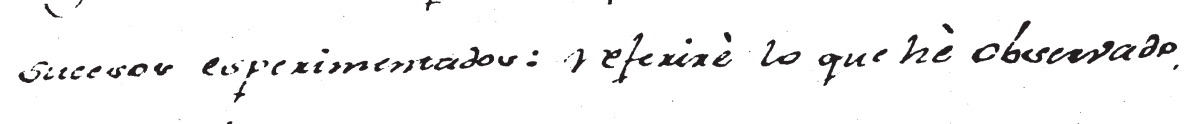

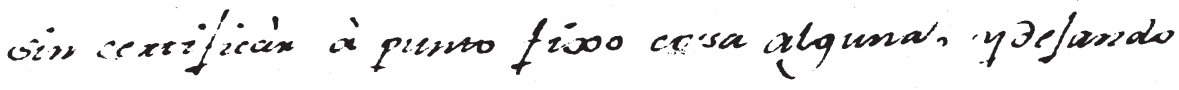

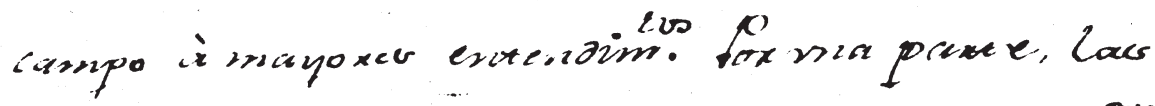

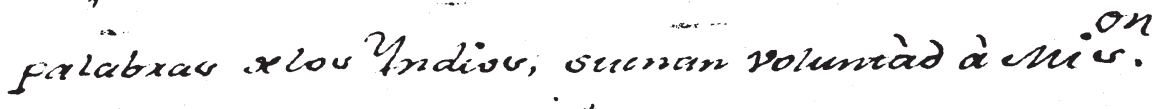
e' hases perido remitidos kavoa aqui it we lis" 


\section{[fol. 2v]}

\{top margin center\} $\backslash 404$

\{right margin\} 172

de los que no pude alcanzàr que se fueran à donde pudieron christianamente vivir: dieronme noticia deotros cinco, que no quisieron parecèr.

II El dia primeiro de mi destino, bapticè, sub condictione in articulo mortis, à un Yndio, y una Yndia de mediana edàd, hermanos del mencionado Capitàn, de los que sus Parientes me aseguraron que deseavan sèr christianos, por no poderlo averiguàr de $\operatorname{los} \mathrm{Pa}\left(\wedge^{\wedge} \mathrm{s}\right)$ cientes, à causa de estàr privados de los sentidos. La mugèr luego murió, à la que no di eclesiastica sepultura por $\left({ }^{\wedge} q\right)$ ue lo reusò [recusó] su parentela; sobre lo que no instè mucho, esperando practicarlo en mejór ocasiòn. Del hombre no supe mas, por que los de su familia se retiraron luego de nuestra cercanía: èste ès el fruto, aunque tan incierto de mi exercicio.

II EN orden à certificàr lo que hè percivido en nuestro negocio, no me ès posible, por la contrariedàd de $\left({ }^{\wedge} \operatorname{los}\right)$ sucesos esperimentados: y refirirè lo que hè observado sin certificàr à punto fixo cosa alguna, y dejando campo à mayores entendimentos. Por una parte, las palabras de los Yndios, suenan voluntàd à Mision el havèr venido remetidos hasta aqui à este fin 
nogen ate $40 s^{\circ}$ te 173

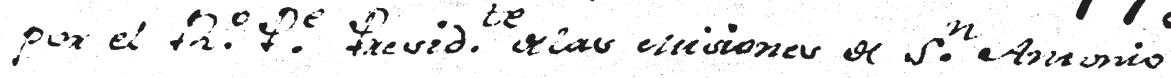

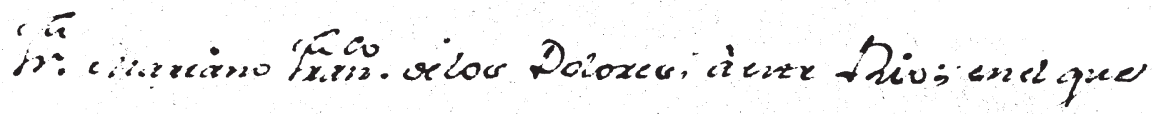

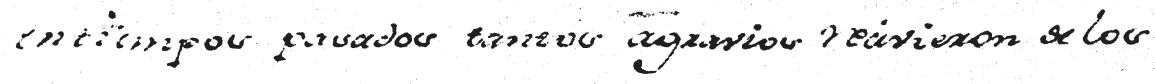

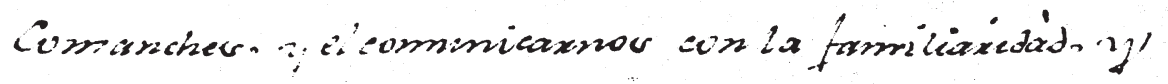

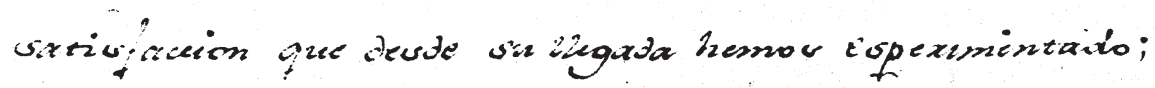

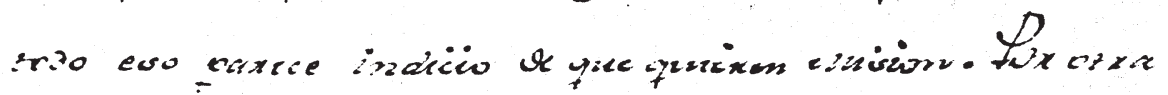

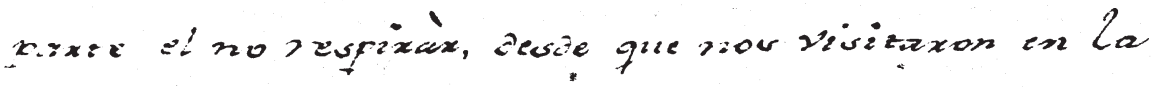

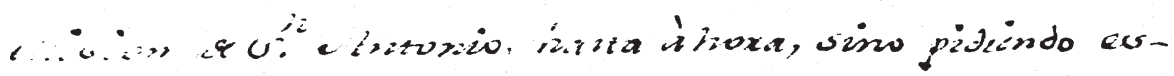

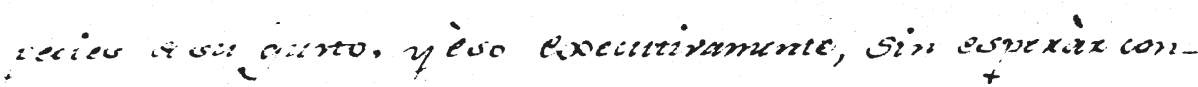

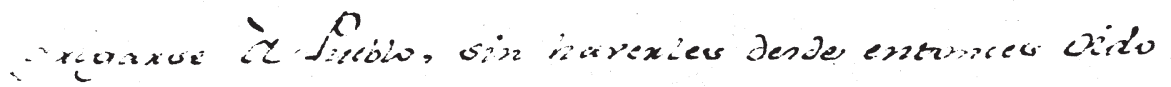

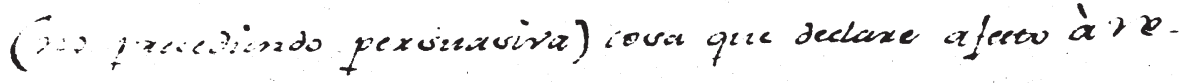

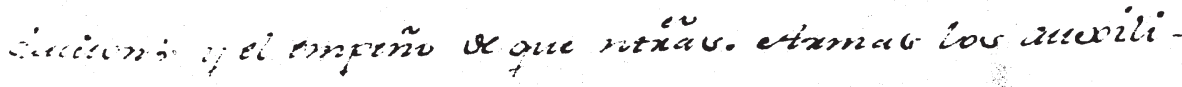

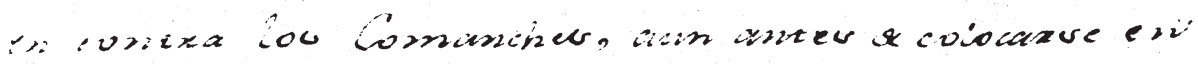

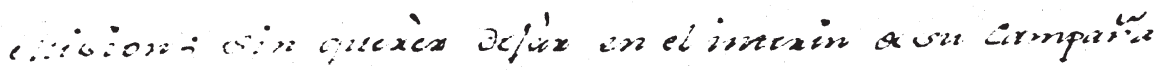

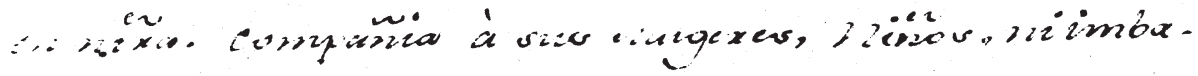

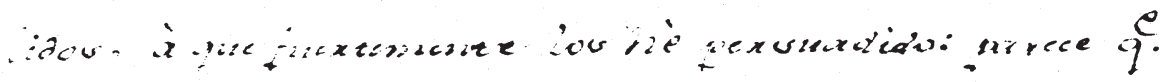

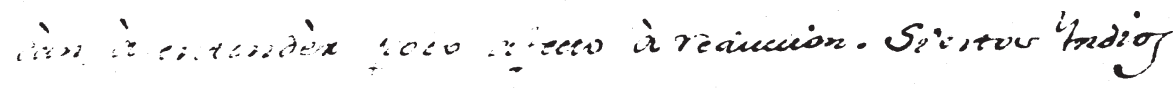

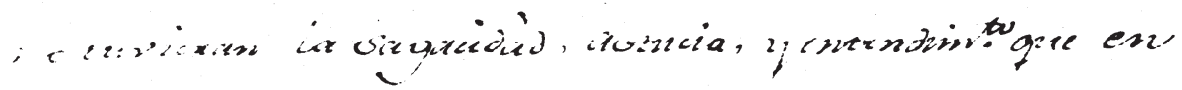

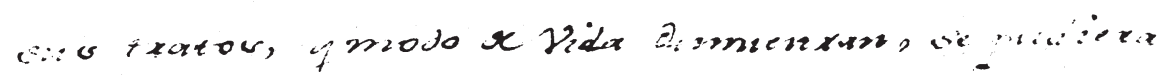

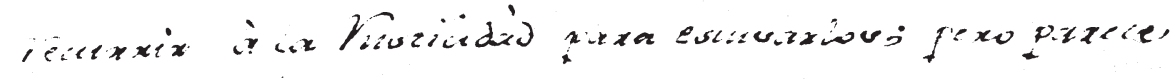

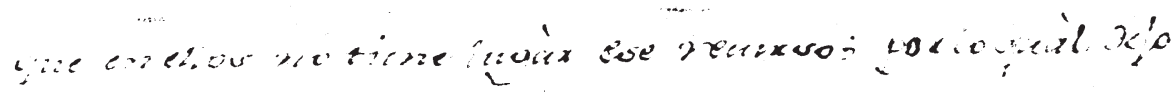

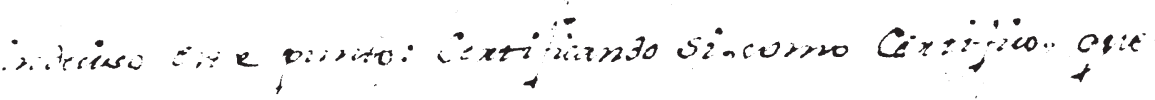


[fol. 3r]

\{top margin center $\} \backslash 405$

\{right margin\} 173

por el Reverendo Padre Presidente de las Misiones de San Antonio

Fray Mariano Francisco de los Dolores; à este Rio; en el que

en tiempos pasados tantos agravios recivieron de los

Comanches, y el comunicarnos con la familiaridàd, y

satisfaccion que desde su llegada hemos esperimentado;

todo eso parece indicio de que quieren Mision. Por otra

parte el no respiràr, desde que nos visitaron en la

Mision de San Antonio, hasta à hora, sino pidiendo es-

pecies de su gusto, y èso executivamente, sin esperàr con-

grègarse à Pueblo, sin haverles desde entonces oido

(no precedindo persuasiva) cosa que declare afecto à re-

duccion; y el empeño de que nuestras Armas los auxili-

en contra los Comanches, aun antes de colocarse en

Mision; sin querèr dejàr en el interin de su campaña

en nuestra compañia à sus Mugeres, niños, ni imba-

lidos, à que fuertemente los hè persuadido: parece que

dàn à entendèr poco afecto à reduccion. Si estos Yndios

no tuvieran la sagacidàd, astucia, $\mathrm{y}$ entendimento que en

sus tratos, y modo de vida [???]entran [demonstran], se pudiera

recurrir à la rusticidàd para escusarlos; pero parece

que en ellos no tiene lugàr ese recurso; por lo quàl dejo

indeciso este punto: certificando si, como certifico, que 


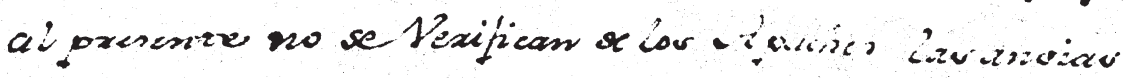

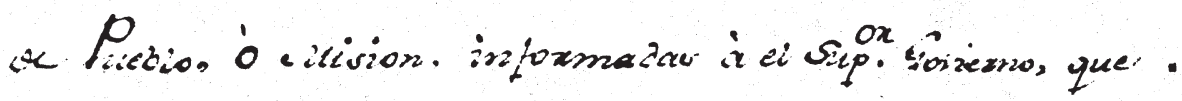

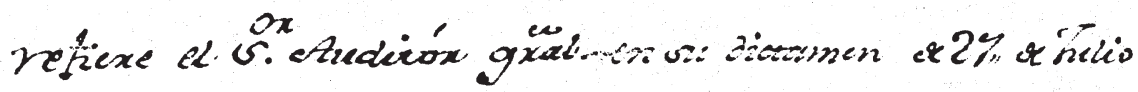

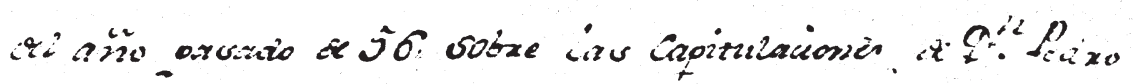

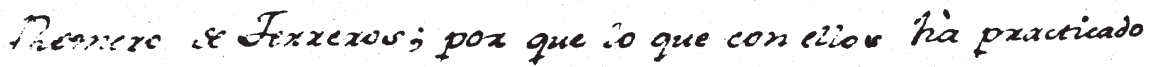

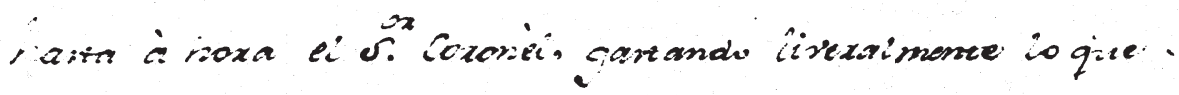

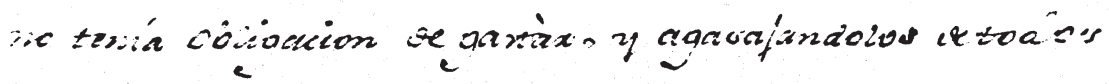

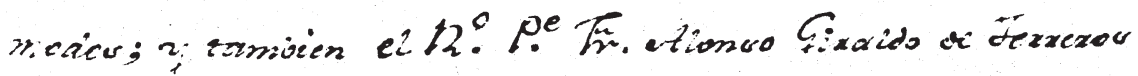

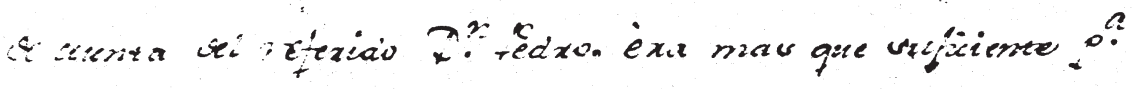

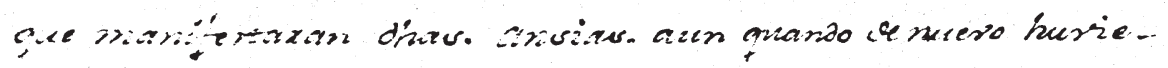
xan sicio erriciaicu, especiaimente vienduros yá en sus tic-

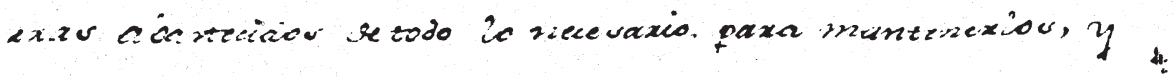

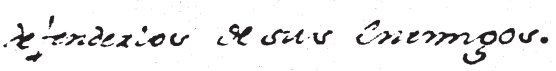

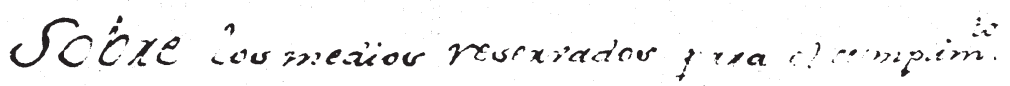

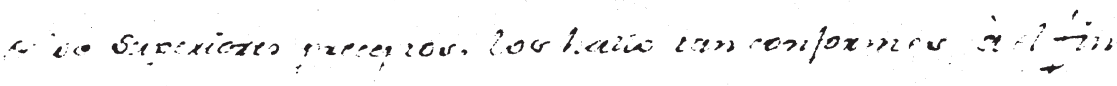

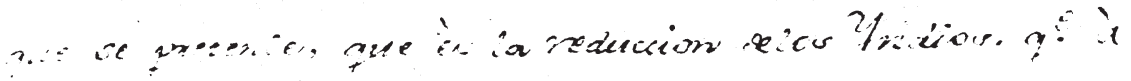

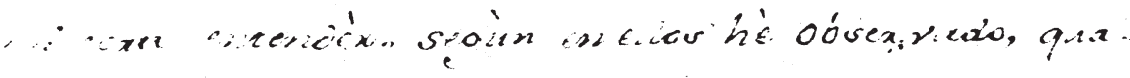

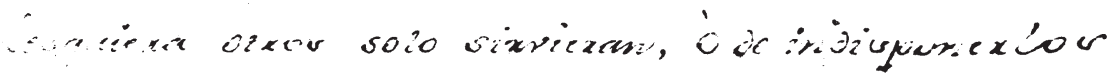

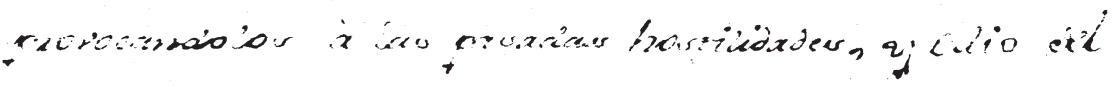

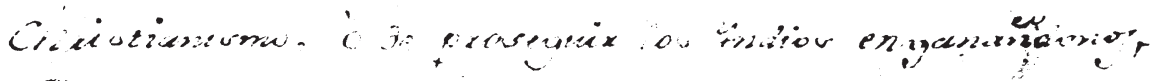

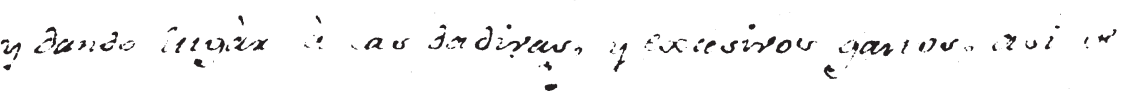

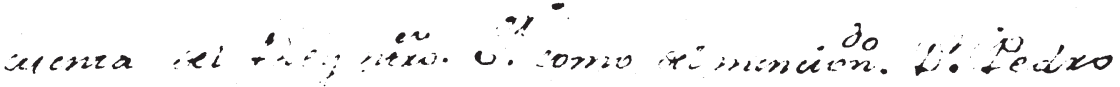


[fol. 3r)

\{top margin cente $\backslash \backslash 406$

\{right margin\} 174

al presente no se verifican de los Apaches las ansias

de Pueblo, ò Mision, informadas à el Superior Govierno, que

refiere el Señor Auditór geñeral en su dictamen de 27,, de Julio

del año pasado de 56,, sobre las Capitulaciones, de Don Pedro

Romero de Terreros; por que lo que con ellos hà practicado

hasta à hora el Señor Coronèl, gastando liveralmente lo que

no tenía obligacion de gastàr, y agasajandolos de todos

modos; y tambien el Reverendo Padre Fray Alonso Giraldo de Terreros

de cuenta del referido Don Pedro, èra mas que suficiente para

que manifestaran dichas ansias, aun quando de nuevo huvie-

ron sido conocidos, especialmente viendonos yà en sus tie-

rras abastecidos de todo lo necesario para mantenerlos, $\mathrm{y}$

defenderlos de sus enemigos.

II SOBRE los medios reservados para el cumplimento

de los superiores preceptos, los hallo tan conformes à el fin

que se pretenden, que ès la reduccion de los Yndios, que à

mi corto entendèr, segùn en ellos hè observado qua-

lesquiera otros solo sirvieran, ò de indisponerlos

provocandolos à las pasadas hostilidades, y odio del

Christianismo, ò de proseguir los Yndios enganañdonos,

y dando lugàr à las dadivas, y excesivos gastos, asi de

cuenta del Rey nuestro Señor como del mencionado Don Pedro 


$$
\text { tol }
$$

y exzxois; pox is que me penuruads à que. Diou ha di.

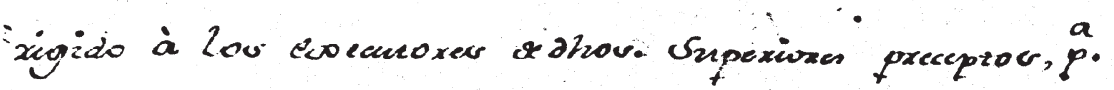

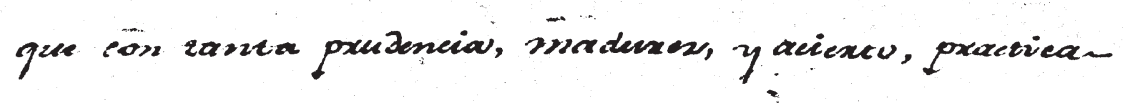
xan Las Jiligniear g" orupectisamente leo pexenecen,

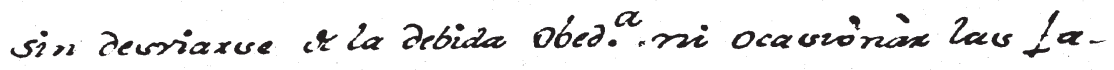

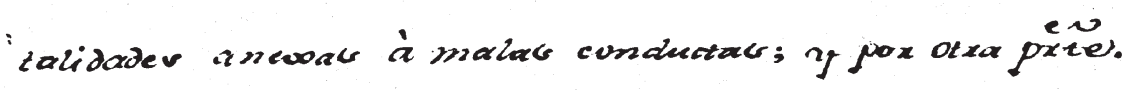

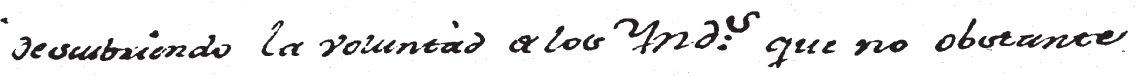

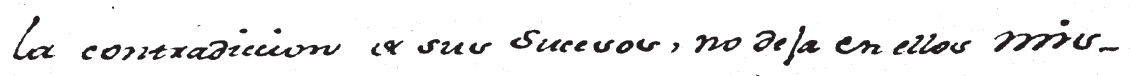
mov a respiandecex. Yaunque al presente no ve pe-

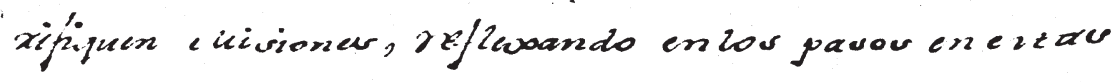

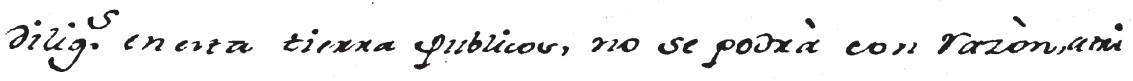

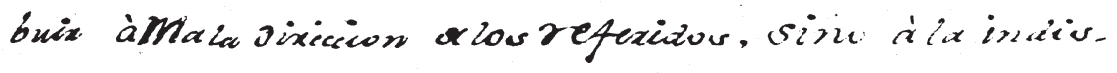

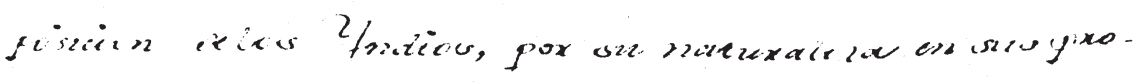

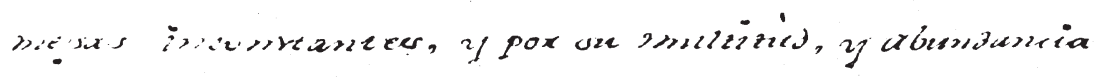

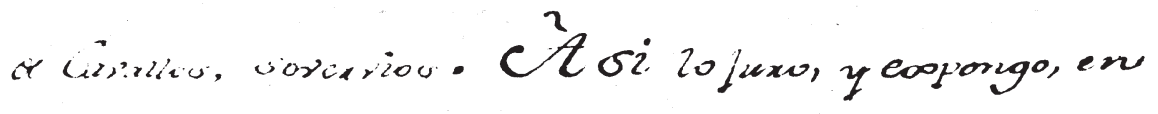
Satiugacion al Pohoxw axisa refoxido, enerce nio san sara, en li diaw vel mes ve balilo

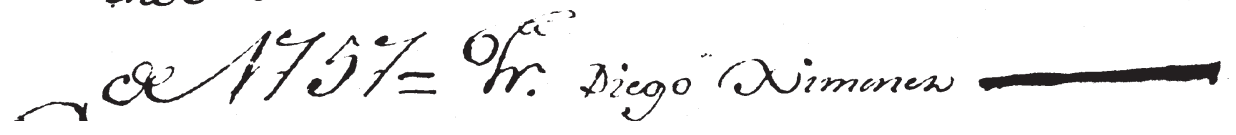


[fol. 3v)

\{top margin center\} $\backslash 407$

\{right margin\} 175

y deotros; por lo que me persuado à que Dios ha di-

rigido à los executores de dichos superiores preceptos, para

que con tanta prudencia, madurez, y acierto, practica-

ran las diligencias que respectivamente les pertenecen,

sin desviarse de la debida obediencia ni ocasiònàr las fa-

talidades anexas à malas conductas; y por otra parte

descubriendo la voluntàd de los Yndios que no obstante

la contradiccion de sus sucesos, no deja en ellos mis-

mos de resplandecèr. Y aunque al presente no se ve-

rifiquen Misiones, reflexando en los pasos en estas

diligencias en esta tierra publicos, no se podrà con razòn, atri

buir à Mala diriccion [dirección] de los referidos, sino à la indis-

posicion de los Yndios, por su naturaleza en sus pro-

mesas inconstantes, y por su multitùd, y abundancia

de Cavallos, sovervios. ÀSI, lo juro, y expongo, en

satisfaccion del exhorto arriva referido, en este

Rio de San Sava, en 4,, dias del mes de Junio

de 1757 = Fray Diego Ximenez 


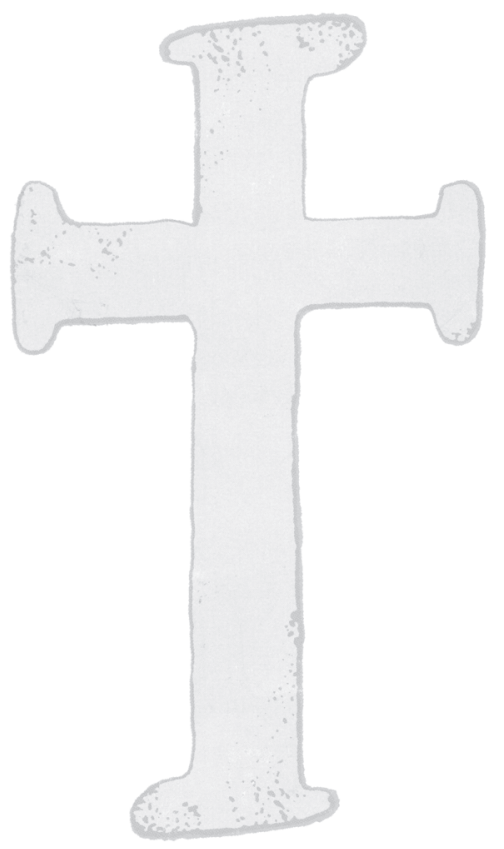




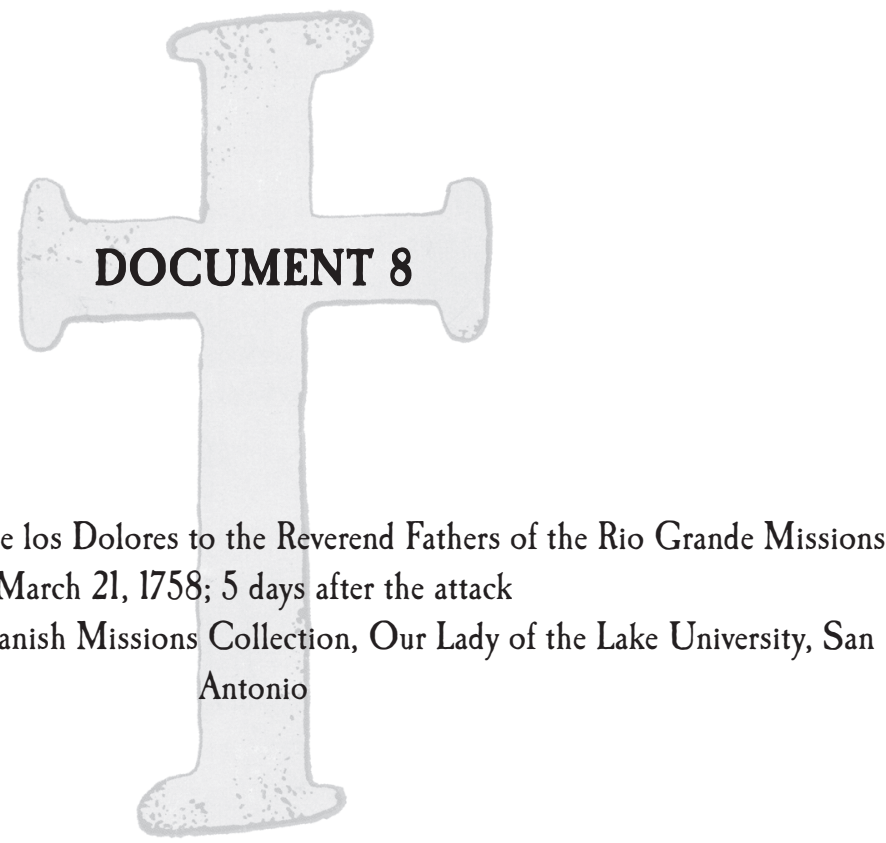




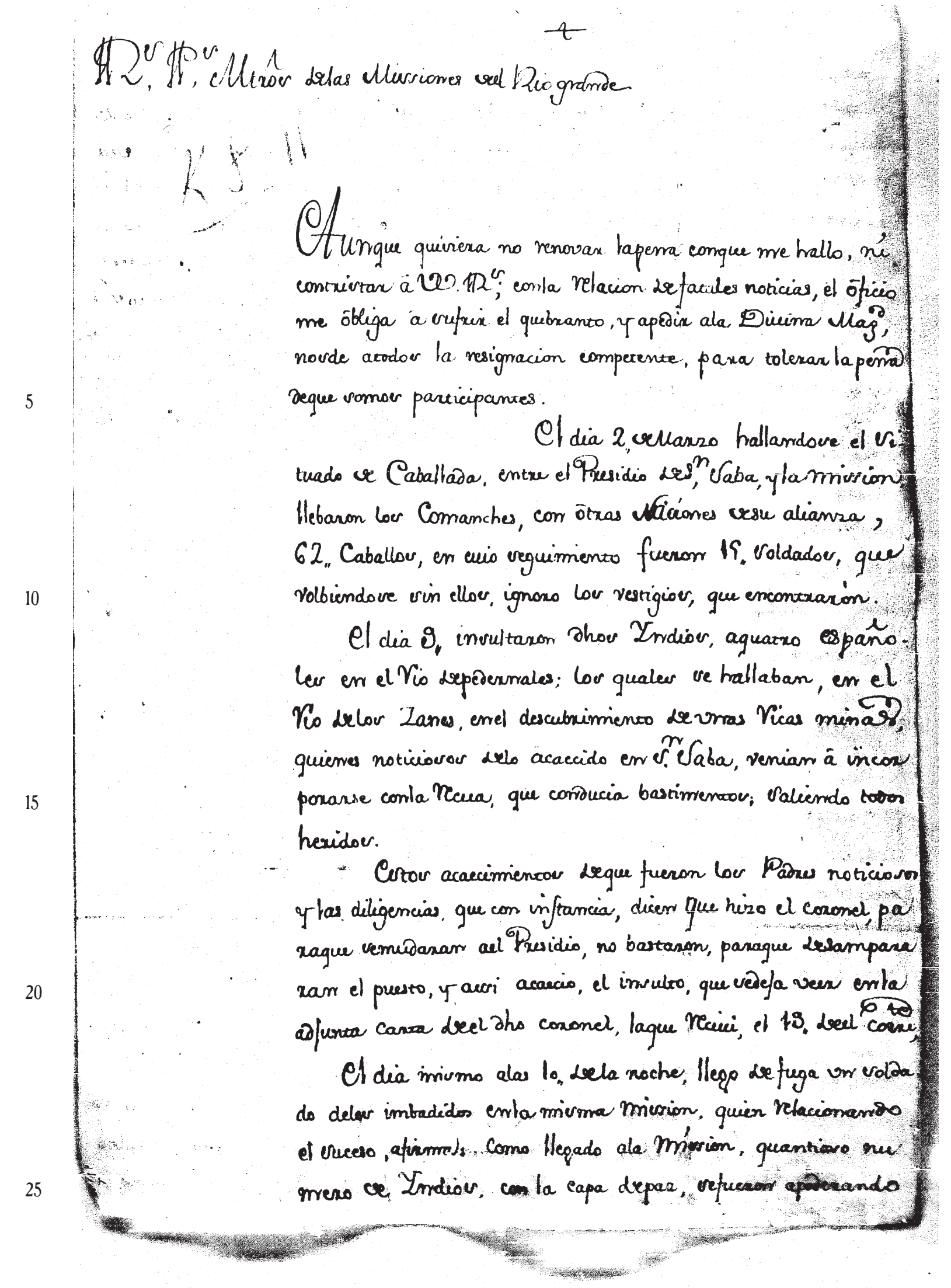


[fol. lr]

\{Heading\} Reverendos Padres Ministrôs delas Missiones del Rio grande

\{Archival reference left margin\} K8-11

Aunque quisiera no renovar lapena conque me hallo, ni

contristar â Vuestras Reverencias; conla relacion de fatales noticias, el ôficio

me ôbliga â sufrir el quebranto, y apedir ala Divina Magestad,

nosde atodos la resignacion competente, para tolerar la peña

de que somos participantes.

II El dia 2,, deMarzo hallandose el si-

tuado de Caballada, entre el Presidio deSan, Saba, y la Mission

llebaron los Comanches, con ôtras (^Na)cíones desu alianza,

62 , Caballos, en cuio seguimiento fueron 15 ,, soldados, que

volbiendose sin ellos, ignoro los vestigios, que encontraròn.

II El dia 9,, insultaron dichos Yndios aquatro españo-

les en el Rio depedernales; los quales se hallaban, en el

Rio delos Zanes, enel descubrimiento de unas Ricas minas, quienes noticiosos delo âcaecido en San Saba, venian â incor

porarse conla Recua, que conducia bastimentos; saliendo todos

heridos.

II Estos acaecimientos deque fueron los Padres noticiosos

y las diligencias que con instancia, dicen que hizo el coronel pa

raque semudaran ael Presidio, no bastaron, paraque desampara

ran el puesto, y assi acaecio, el insulto, que sedeja ver en la

adjunta carta deel dicho coronel, laque recivi, el 19,, deel corr[iente];

II El dia mismo alas 10,, dela noche, llego de fuga un solda-

do delos imbadidos enla misma mission, quien relacionando

el suceso, afirmando como llegado ala mission quantioso nu

mero de Yndios, con la capa depaz sefueron apoderando 
Leel puesto, y alqunoer bienses, gue cogian, loque se dirimutaba porno causarles dingueroo, havtague resucleos, aconoucixlos al Previdio, aum ellor rsiumor, acridaxon á encillax el Caballo de el R.P. Pres; quien moniado, al invtance deralix, 6 2exsi baxon à balaerour; quedando en el mirmo lanse muexto, el pe, P. Sariesteban, y unvoldado, yel P.P. Molima, con un bxaso quebrado. At invtante deipolaxion low Zndios, alos Pazxes it,

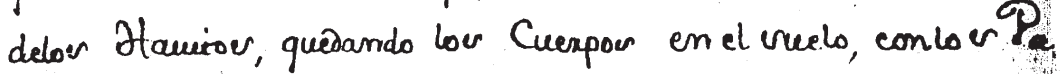
nor menow, y varratias. el P, Hexido, con cinco voldador, do

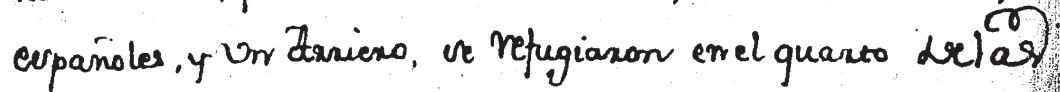
Cargas, endonde estubienvor resivtiendo, todo el dia 16, yaquells. noche, querando enla contienta, lor mas muentos, y lor menop haidos, Lesueste, que haviendo guedado el P. Ir. lliguel, con Nit. colas el ctrrierso, y Un voldado, dretexminasuon la fuga, por un

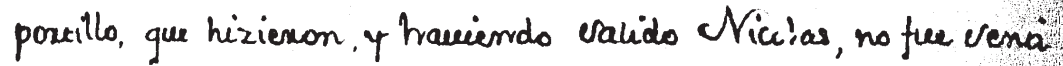
do drelor Irrdiow. ( pero ceefte no vabemor el fin) Valio tambin el Voldado, con el Padxe (quienr desdeluego ve hallaria mue farigado) pues el voldado lo llebata cangado, pess quizo la desgracia, gue 10 vintieran lor Indioer, y lesrizawers algunor balssov, delos 20 que alcanzarridef uno, Cais el voldado sim vernaido, dreel que recobrado, ni vido al Pazre, ni vupo veel mas; yes decentix, ques vi tho ${ }^{6}$, no murio, velo llebaxien caurito. Pox aca ve trase quicio, seguen ta veñal, que rierre tho voldado dela bala, en tas efpaldas, que traespars al Parre, trdo el cuinpo, ponlogue nole guedo. feersa, para trazen maion estrago in tho esoldado. yque este.

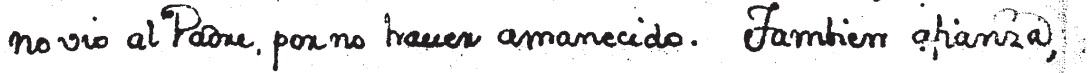
québaban tas mugeses y niños, niver, aum tra via, yquelos

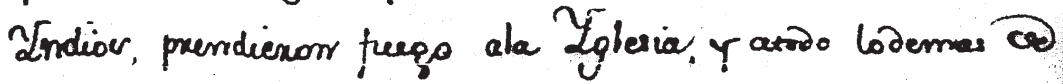

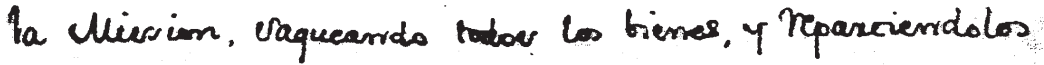

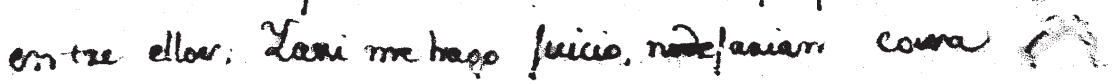


deel puesto, y algunos bienes, que cogian, loque se disimulaba, porno causarles disgusto, hastaque resueltos, aconducirlos al Presidio, aun ellos mismos, auidaron â encillar el Caballo de el Reverendo Padre Presidente; quien montado, al instante desalir, lo derri baron â balasos; quedando en el mismo lanze muerto, el Padre

Predicador Satisteban, y un soldado, y el Padre Predicador Molina, con un brazo quebrado. $\mathrm{Al}$ instante despojaron los Yndios, alos Padres delos Havitos, quedando los Cuerpos en el suelo, conlos $\mathrm{Pa}$ ños menores, y sandalias. El Padre Herido, con cinco soldados, dos españoles, y um Arriero, se refugiaron en el quarto, delas cargas, en donde estubieron resistiendo, todo el dia 16, yaquella noche, quedando enla contienda, los mas muertos, y los menos heridos, desuerte, que haviendo quedado, el Padre Fray Miguel, con Nicolas el Arriero, y un soldado, determinaron la fuga, por un pozeillo, que hizieron, y haviendo salido Nicolas, no fue senti do delos Yndios, (pero deeste no sabemos el fin) salio tambien el soldado, con el Padre (quien desdeluego se hallaria mui fatigado) pues el soldado lo llebaba cargado, pero quizo la desgracia, que lo sintieran los Yndios, y les tirasen algunos balasos, delos que alcanzandoles uno, caio el soldado sin sentido, deelque recobrado, ni vido al Padre, ni supo deel mas; y es desentir, que si dicho Padre no murio, selo llebarian cautibo. Por aca se haze juicio, segun la señal, que tiene dicho soldado dela bala, en las espaldas, que traspaso al Padre, todo el cuerpo, porloque nole quedo fuerza, para hazer maior estrago en dicho soldado, yque este no vio al Padre, por no haver amanecido. Tambien afianza, quedaban las mugeres y niños, vivos, aun toda via, yque los Yndios, prendieron fuego ala Yglesia, y atodo lodemas de la Mission, saqueando todos los bienes, y repartiendolos en tre ellos; Yami me hago juicio, nodejarian cossa 
algurra, puer hasto, el Garrado, que estaba errel conxal, estabin macando. Fodo esto lo relaciona el voldado, racticamoines en ello, como tesigo óculan, y afianzands, traces to vecesti do, enta forma expressada Lel Presides trasta oy, no hill venido mas rason, puer vnicamente Ulegaxon. los dos voldtes das. Conductones drela Carta deel Cononel, despres ce hasund la Leydo el Themiente, guien dejande enel Campo has Cangag, apreeruxs sce maxctra, con 18. hombxe entue voldados, y tixsienoer, y no vabemoer. vi abra llegado al Presidio.

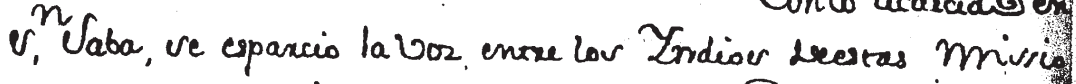

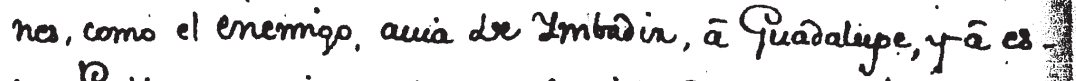
to Rublos, por cuis motivo, ya remin, atruen tora la pobla esor ce Quadalupe, aesta mission, hasta, quetenga el resguax, do corspetente, o vepamos el fin de esta convisatacion. en

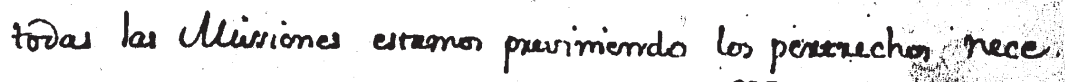
Sarioes, para resiutix conla arida crel or al enernipo Si viniere, cuia mulativo es grande.

ro

On esta Causa, no relolvi

embiain algunor Indion de socoxiro, haciendo jucicio, guéde ix poros, exa cebax al enermip; y de in muchos guadabromo es Centepara la detensa, y expentos a eúónce peliquo, aques vellega, que hauiendo alo viemo, como Soo i, hombres ce axmal

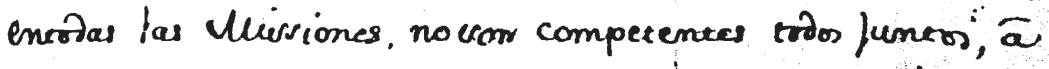
coneraxestax all enempo, err campo raso: Ypueden vero resguaxdados enum miesiones, endoppde has muexis los pximcipales quexseror, y ere tran remplasad, con Irazon Genciles, y bizonoer. se halla el Capitan impendo à vacouner y lehe pas

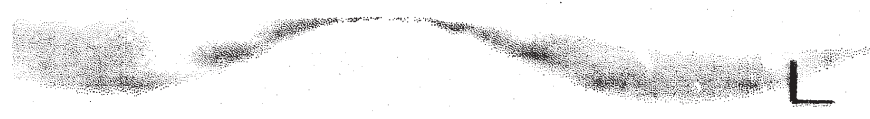


alguna, pues hasta el Ganado, que estaba en el Corral, estaban matando. Todo esto, lo relaciona el soldado, ratificandose en ello, como testigo ôcular, y afianzando, haver todo sucedido enla forma expressada. Deel Presidio hasta oy, no ha venido mas razon, pues unicamente llegaron los dos solda dos, conductores dela carta deel Coronel, despues de haver la leydo el Theniente, quien dejando enel campo las cargas, apresuro su marcha, con 18. hombres entre soldados, y Arrieros, y no sabemos, si abra llegado al Presidio.

II Con lo acaecido en

San Saba, se esparcio la Voz, entre los Yndios deestas Missio nes, como el enemigo, avia de Ymbadir, â Guadalupe, y â estos Pueblos, por cuio motivo, ya remiti átreer toda la poblason de Guadalupe, aesta Mission, hasta que tenga el resguar do competente, ó sepamos el fin de esta conturbacion. En

todas las Missiones estamos previniendo los pertrechos nece sarios, para resistir, con la auida deel Señor ael enemigo si viniere, cuia multitud es grande.

II Por esta causa, no resolvi

embiar algunos Yndios de socorro, haciendo juicio, que de

ir pocos, era cebar al enemigo; y de ir muchos, quedabamos, sin

Gente para la defensa, y expuestos á evidente peligro; aque sellega, que haviendo alo sumo, como 500,, hombres de armas entodas las Missiones, no son competentes todos juntos, â contrarestar ael enemigo, en campo raso: y pueden serlo resguardados en sus missiones, endonde han muerto los principales guerreros, y se han remplazado, con Yndios Gentiles, y bizeños.

II El Presidio, solo tiene 5,, soldados; y assi se halla el Capitan impedido â socorrer, y solo lehe per 


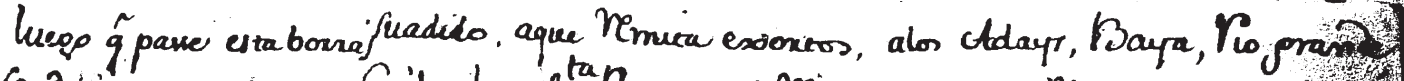

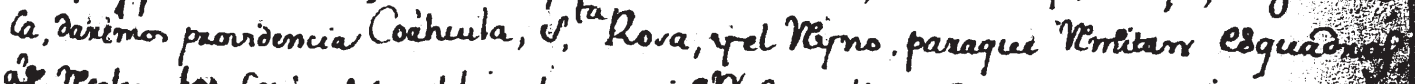

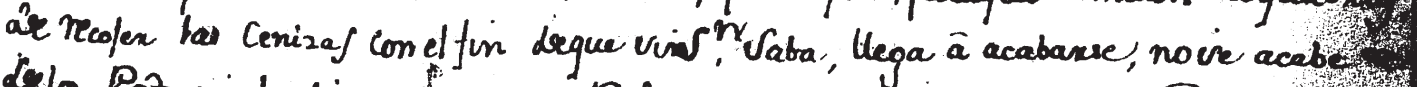

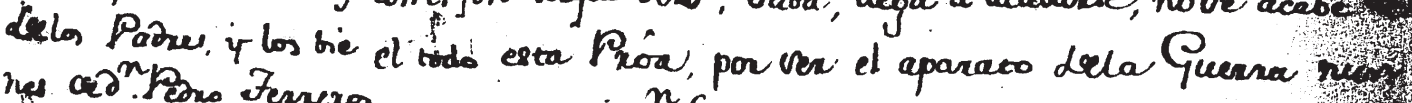

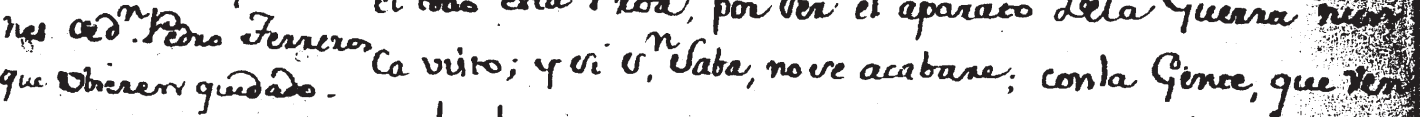
ga, has fuensas, que quedarem; escos pueblos, y la aleans? detpaches, y otxas rracio, repueda castigax, tarr desmos

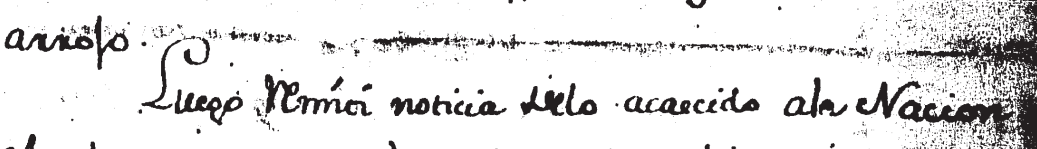
cApache, y aungue me han prometido, el ix cxecido $n$,

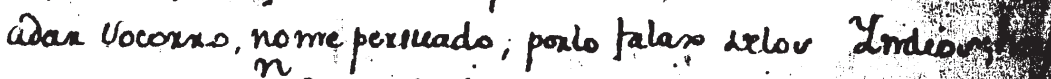
ta vaber de $v_{1}^{n}$ Sata el efeceo desu promersal

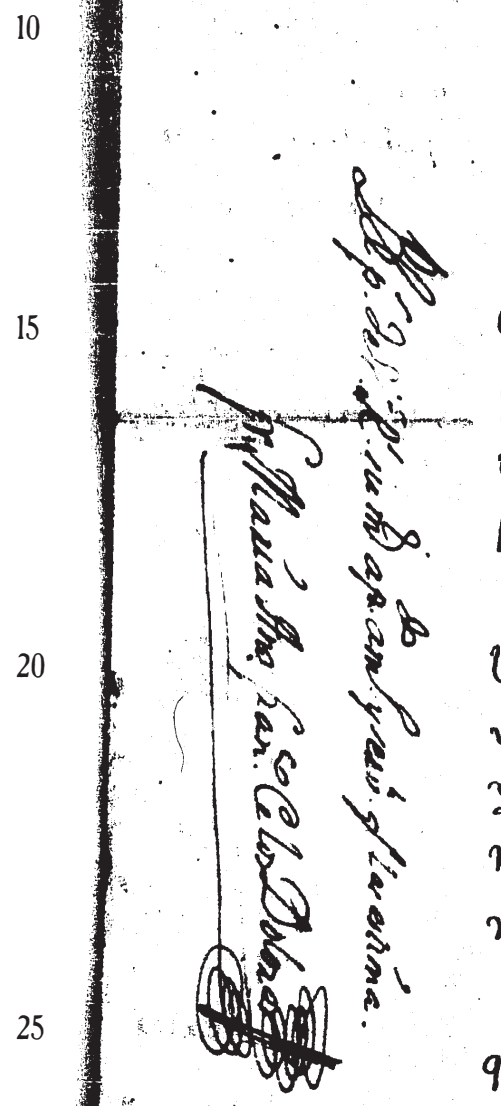

- estereleter.

engue quéramos, yaungue, quiviena noticiando al fot to Guadian, la Uxgemeia ceel rimpo nodalegan, y ot 4 plecs a VO, RR, arbitun modo de remitix esta oby incluera, paraque Ulegue ase noticia, yoers ambo $C_{0}$ lav providencias que les parescain converniences. (1). RR. volicien atoda diligencia una carga deplowity

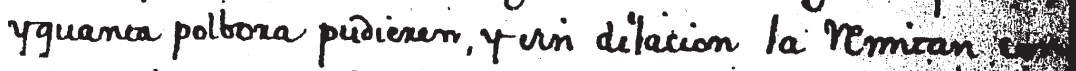
Indios deessias miessiones, pon hallarse estar esceras of municiones, Lentague escriven al Colegio. ve enroty rengan becstos pertrechos para cada miesion. Yo aprecund

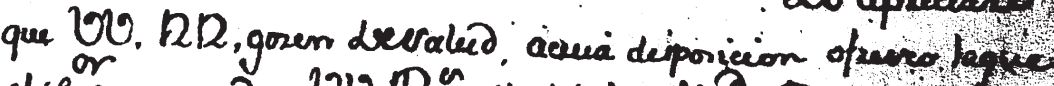

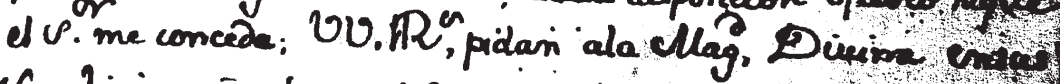

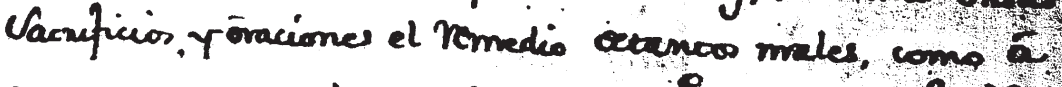
menasam; que yo le vuphicare me g. ous iridas in? as cllicion des Anomio, y cllarso 21 de 1758. 
luego que passe esta borras-

ca, daremos providencia

de recojer las cenizas

delos Padres, y los bie-

nes de Don Pedro Terreros

que ubieren quedado. suadido aque remita exortos, alos Adays, Baya, Rio grande, Coahuila, Santa Rosa, y el Reyno, paraque remitan esquadras con el fin deque $\operatorname{si}\left({ }^{\wedge} \mathrm{S}\right)$ an Saba, llega â acabarse, no se acabe [de] el todo esta Prôa, por ser el aparato dela Guerra nunca visto; y si San Saba, no se acabare; con la Gente, que venga, las fuerzas, que quedaren; estos pueblos, y la alianza deApaches, y otras nacio[nes], sepueda castigar, tan desmedido arrojo.

II Luego remíti noticia delo acaecido ala Nacion

Apache, y aunque me han prometido, el ir crecido numero adar socorro, no me persuado; porlo falar delos Yndios hasta saber de San Saba el efecto desu promessa.

II Este es el estado enque quedamos, y aunque, quisiera noticiarlo al Reverendo Padre Guardian, la urgencia deel tiempo noda lugar, y assi suplico a Vuestras Reverencias, arbitren modo de remitir esta con la inclusa, para que llegue asu noticia, yden ambos Colegios las providencias, que les parescan convenientes.

\author{
II Estimare â \\ Vuestras Reverencias soliciten atoda diligencia, una carga de plombo, \\ y quanta polbora pudieren, y sin dilacion la remitan con \\ Yndios deessas missiones, por hallarse estas escasas de \\ municiones, Yenlaque escrivan al Colegio, se en cargue \\ vengan deestos pertrechos para cada mission. \\ II Yo apreciare \\ que Vuestras Reverencias, gozen desalud, acuia disposicion ofresco laque \\ el Señor me concede; Vuestras Reverencias pidan ala Magestad Divina ensus \\ sacrificios, y ôraciones el remedio detantos males, como á- \\ menazan; que yo le suplicare me guarde sus vidas muchos años. \\ Mission deSan Antonio, y Marzo 21 de 1758.
}




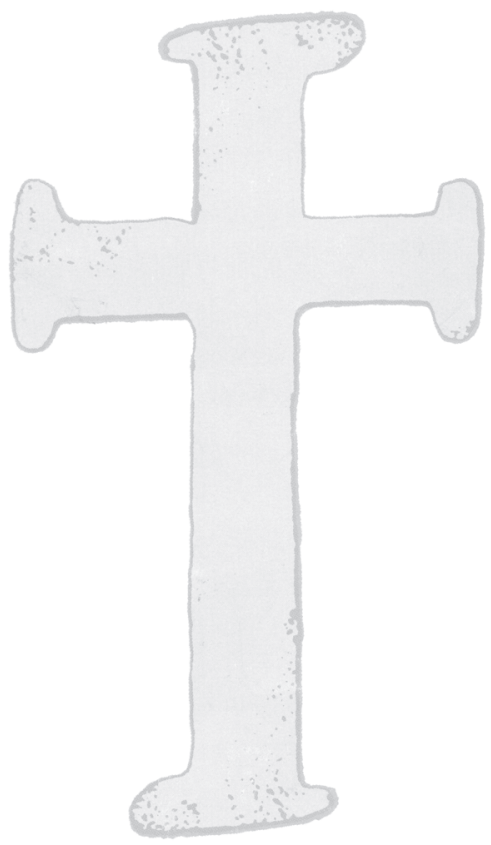




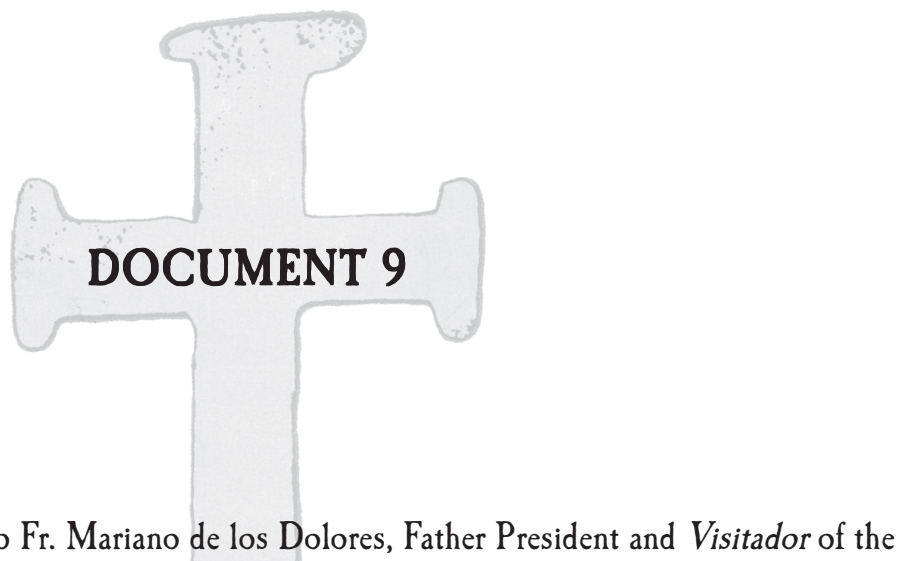

Letter from Colonel Parrilla to Fr. Mariano de los Dolores, Father President and Visitador of the Texas Missions

May 22, 1758; 2 months after the attack

Celaya Collection, Old Spanish Missions Collection, Our Lady of the Lake University, San

Antonio 
Spanish Colonial Documents Pertaining to Mission San Sabá

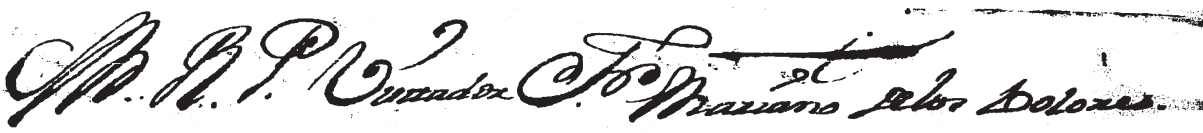

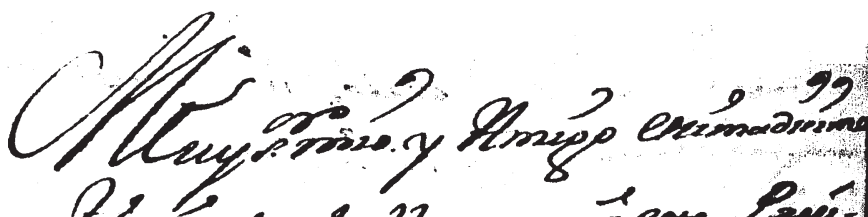

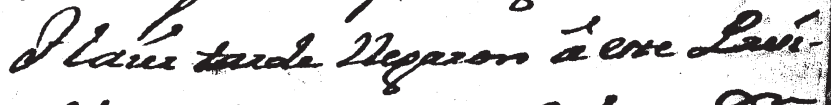

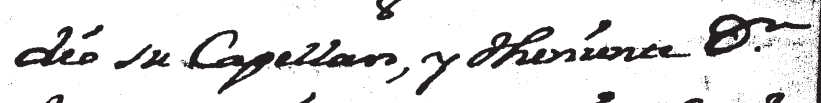

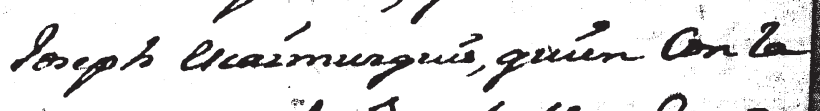

5

10

15

20

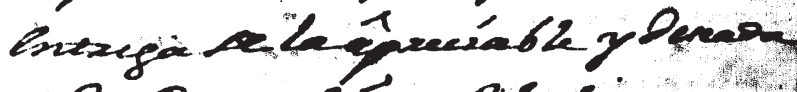
celpg. me kape dibedon te

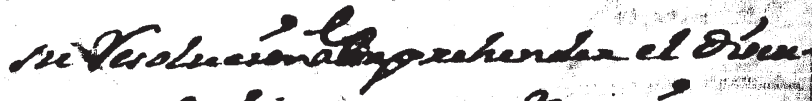

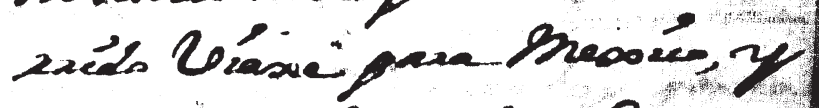

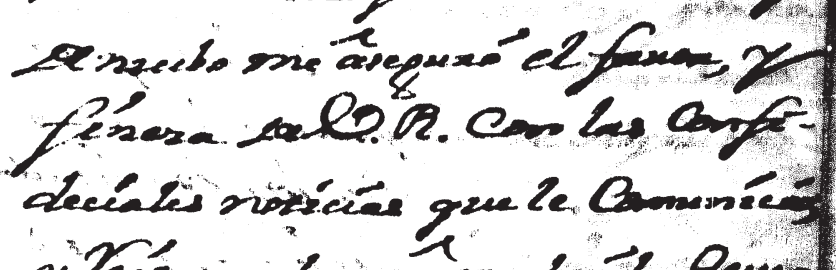

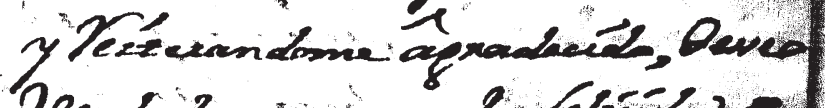

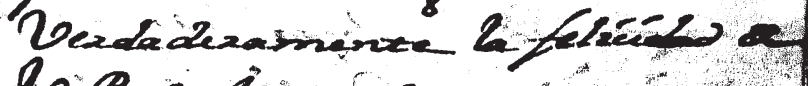
l.R. en is réto.

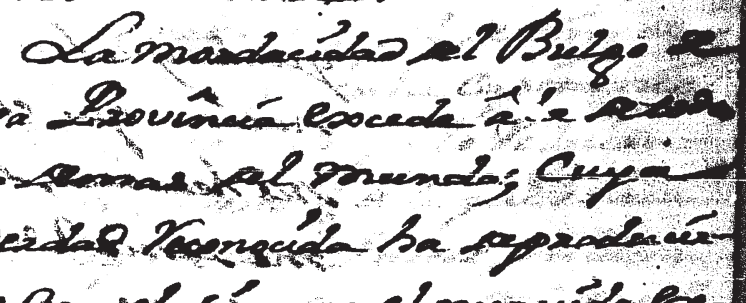

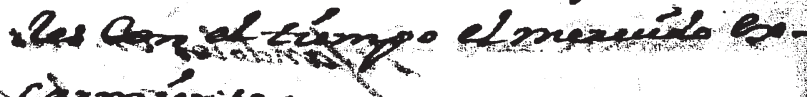

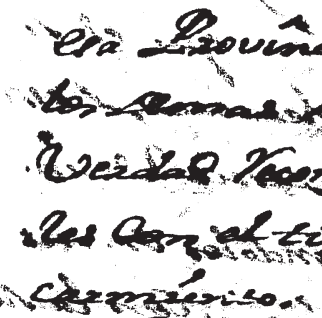

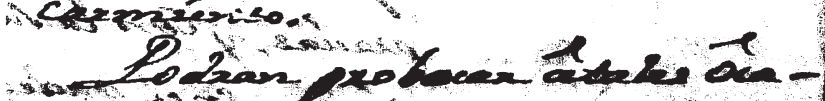

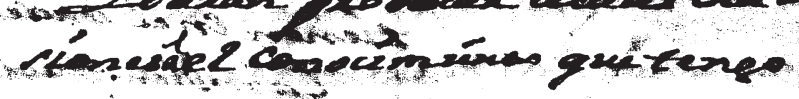

108 
[fol. 1r]

\{Heading\} Muy Reverendo Padre Visitador Fray Mariano delos Dolores

Muy señor mio. y Amigo estimadisimo

Hazér tarde llegaron â este Presí-

dio su Capellan, y Theniente Don

Joseph escaimuzquis, quien con la

entrega de la âpreciable y deseada

de Vuestra Reverencia me hizo sabedor de

su Resoluciona(^em)prehender el discu-

rrido Viaxe para Mexico, y

de nuebo me âseguró el favor, y

fineza de Vuestra Reverencia con las confi-

deciales noticias que le comunicó;

y Reiterandome âgradecido, deseo

Verdaderamente la felicidad de

Vuestra Reverencia en un todo.

II La mordacidad del Bulgo de

esa Província excede â la detodo

los demas del mundo; cuya

Verdad Reconocida ha deproducir-

les con el tiempo el merecido ex-

carmiento.

II Podran probocar âtalas ôca-

sionesâel conocimiento que tengo 
Spanish Colonial Documents Pertaining to Mission San Sabá

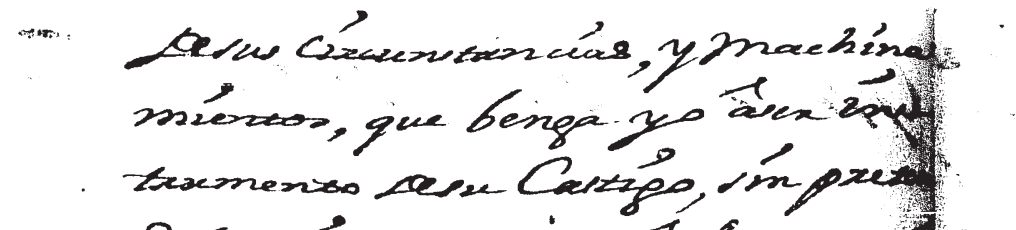
dexle, né Coxxupondex à Lapropin? mia, lasa Dczpación tence fariet

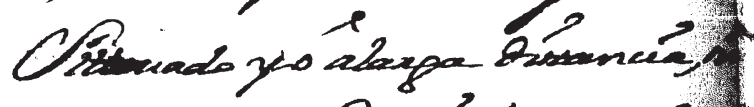

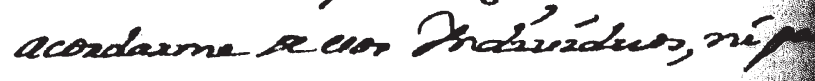
ha buers, ne para nato, Comertil dewise, no he Consyunde libevenmé Leviona de la Caleumnea, ne 8

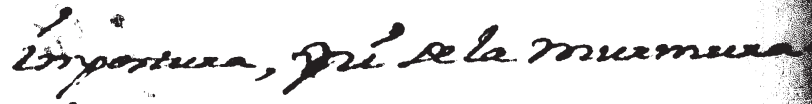
ceá

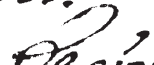

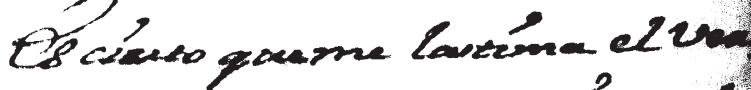

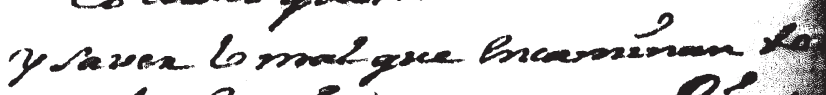

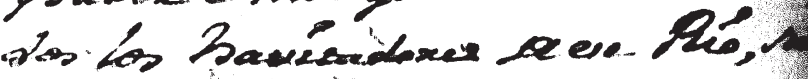
Zlegar àony bxpa y Deralichada Ditiajpero nalum

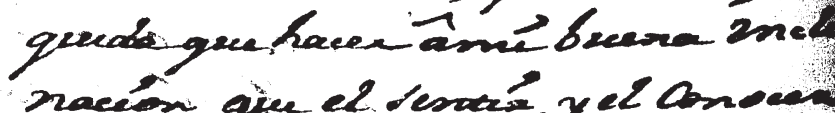

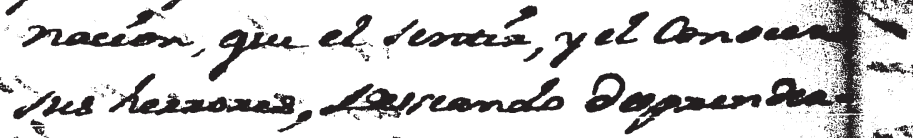

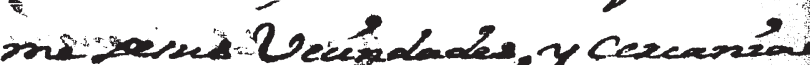
to

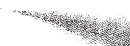


[fol. lv]

de sus circunstancias, y machina-

mientos, que benga yo âser ins-

trumento desu Castigo, sin preten-

derlo, ni corresponder â lapropension

mia, una ôcupacion semejante.

II $\operatorname{Sit}(\wedge$ tu)ado yo âlarga distancia, sin acordarme de esos Individuos, ni para bueno, ni para malo, como suele decirse, no he conseguido libertar mi Persona de la calumnia, de la impostura, (^n)i de la murmuracion.

II Es cierto que me lastima el ver y saver lo mal que encaminan todos los havitadores de ese Rio, sus proiectos, y sus diligencias para llegar â mejorar y âlibiar una desdichada Patria; pero nada mas queda que hacer âmi buena inclinacion, que el sentir, y el conocer sus herrores, desseando desprenderme de sus Vecindades, y cercanias dejandoles en sus bien recividas desgracias, y con la satisfaccion que tienen en el padecer miserias.

II Tambien pudo âsegurar â Vuestra Reverencia 


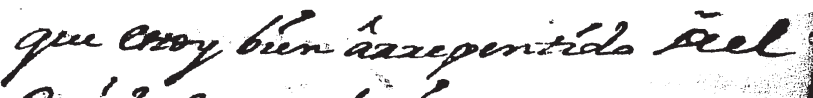
Qceidads grie he zintexprento

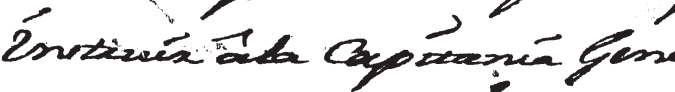

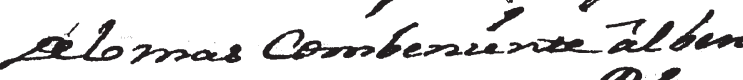
to favoube expos Do Q

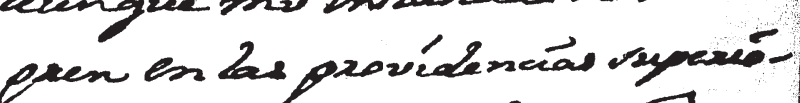

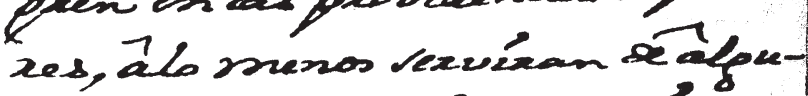
na Lur, of Cosseno para zonar

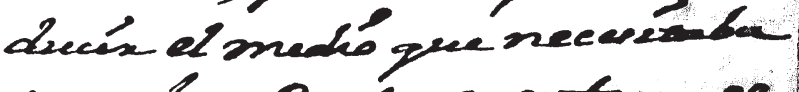

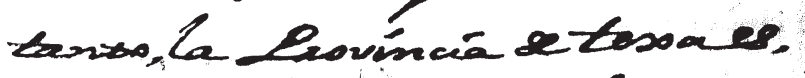

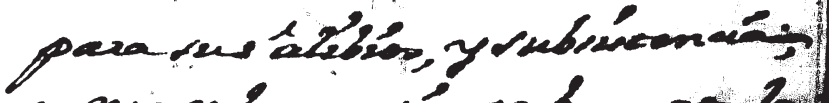

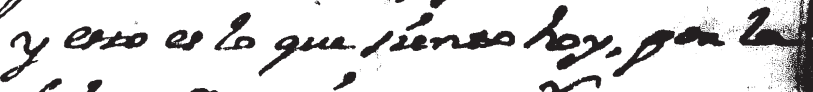

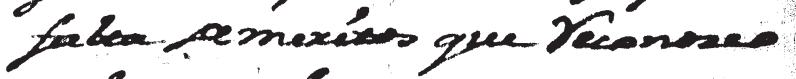

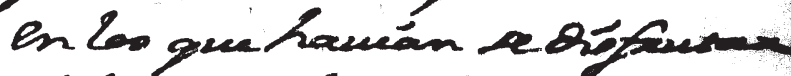
el bien, yelkeng ieio.

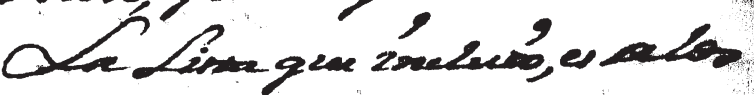

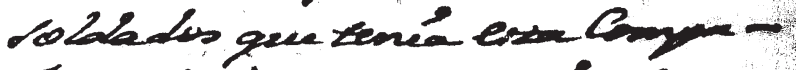

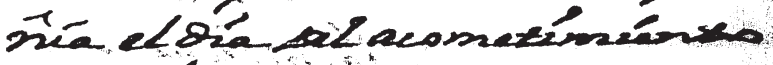

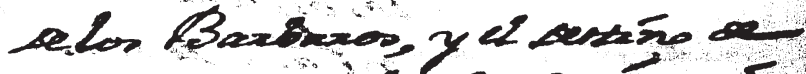

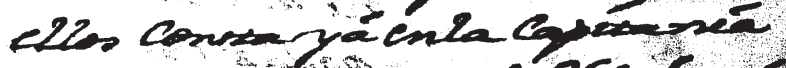

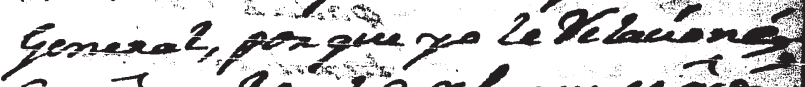

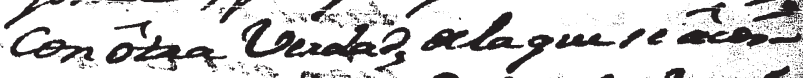

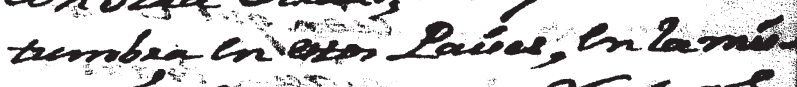

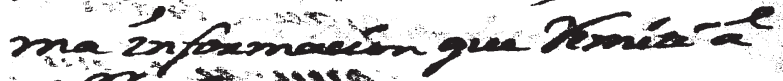

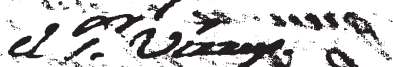

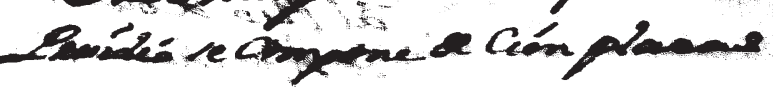




\section{[fol.2r]}

que estoy bien ârrepentido deel

cuidado que he interpuesto para

instruir âla Capitania General

delo mas combeniente âl bene-

ficio, y favor de estos Paises: pues

aunque mi instancias no se lo-

gren en las providencias superio-

res, âlo menos serviran de âlgu-

na Luz, y Camino para intro-

ducir el medio que necesitaba

tanto, la Provincia de texas,

para sus âlibios, y subsistencia;

y esto es lo que siento hoy, por la

falta de meritos que reconozco

en los que havian de disfrutar

el bien, y el beneficio.

II La Lista que incluio, es delos soldados que tenia esta Compañia el dia del acometimiento de los Barbaros, y el destino de

ellos consta yá enla Capitania

General, por que yo le relacioné, con ôtra Verdad, dela que se âcostumbra en estos Paises, en la misma informacion que remiti â

el señor Virrey.

II Sabe muy bien Vuestra Reverencia que este

Presidio se compone de cién plazas 


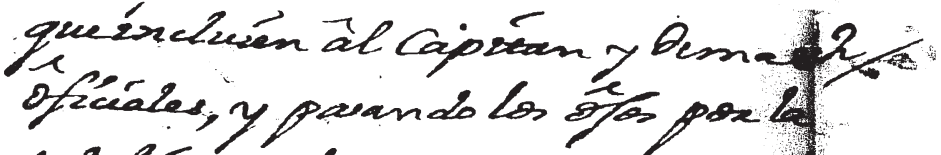

$$
\begin{aligned}
& \text { tal Lista, de hallaxá gue exaba e } \\
& \text { sucmexs Conplexis, yque eres edf }
\end{aligned}
$$

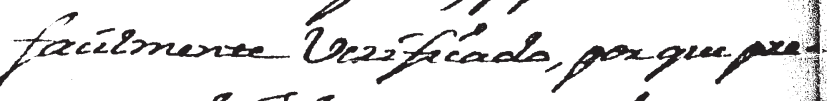
prencando álos gee re nombram en

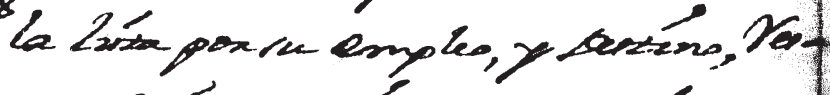
pondexion precuenomente que hexan.

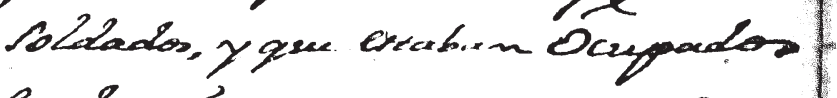
en Lomino que yo tengo expen.

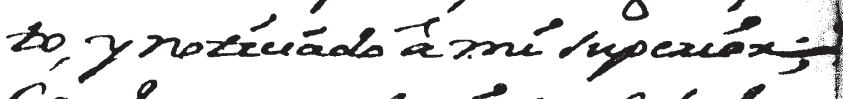

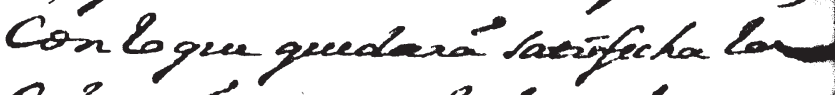

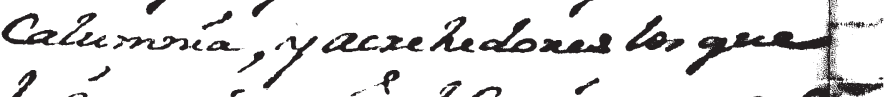

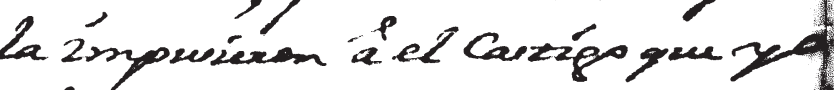

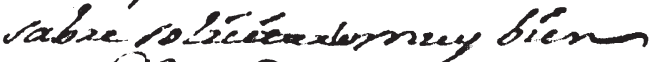
Dlos Brateos axpenoxes, noloo

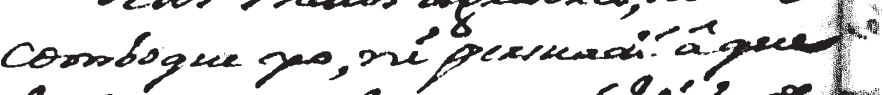
Lfrueren en el prenzo, né Lida de

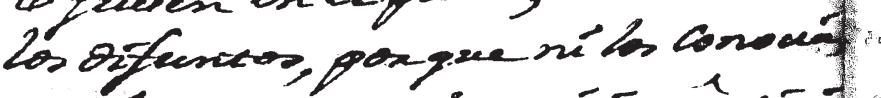

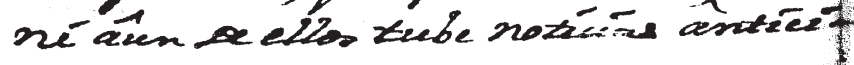
padas; Congice por en pares engl

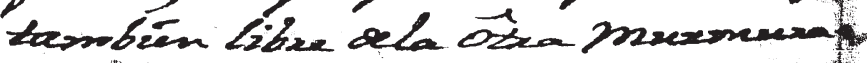
cion gue Curale ence cos Pintecos

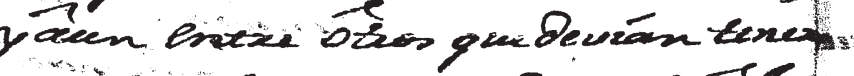

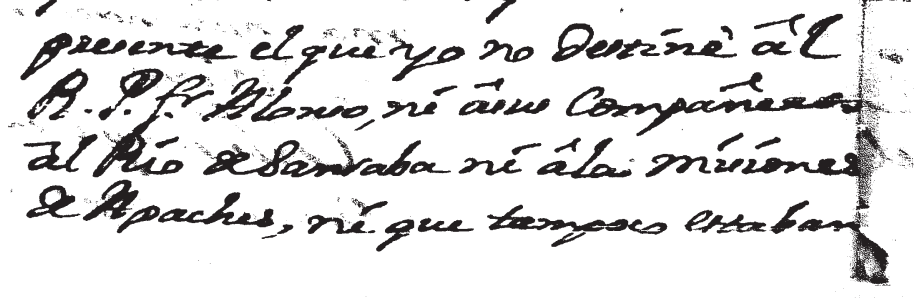


[fol. 2v]

\{right margin\} 2

que incluien àl Capitan y demas

ôficiales, y pasando los ôjos por la

tal lista, se hallará que estaba el

numero completo, y que esto es

facilmente verificado, por que pre-

guntando âlos que se nombran en

la lista por su empleo, y destino, res-

ponderian precisamente que heran

soldados, y que estaban ôcupados

en lomismo que yo tengo expues-

to, y noticiado â mi superior;

con lo que quedará satisfecha la

calumnia, y acrehedores los que

la impusieron â el castigo que yo

sabre solicitarlesmuy bien

II Alos Yndios âgresores, no los

comboque yo, ni persuadi âque

le fuesen en el puesto, ni Vidas de

los difuntos, por que ni los conocia,

ni âun de ellos tube noticias ântici-

padas; con que por esta parte estoy

tambien libre dela ôtra murmura-

cion que cunde entre esos Rusticos,

y âun entre ôtros que devian tener

presente el que yo no destiné âl

Reverendo Padre Fray Alonso, ni âsus compañeros

âl Rio de Sansaba ni âlas misiones

de Apaches, ni que tampoco estaban 


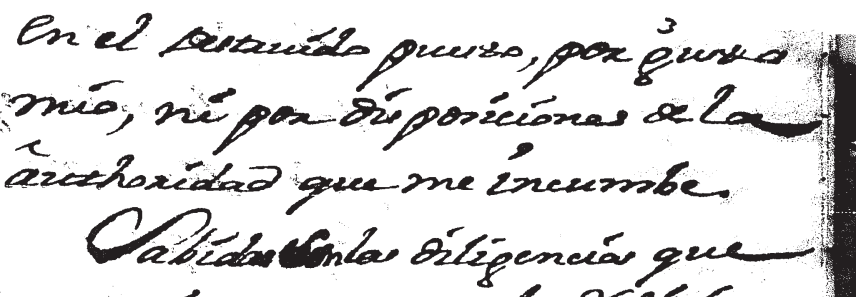

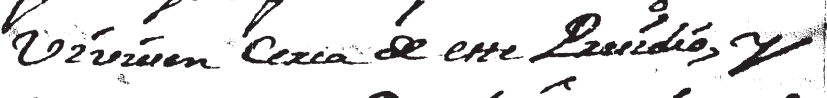
lon notoxía bolins las axtrucínes meas Con un Lewanasy y Con pus

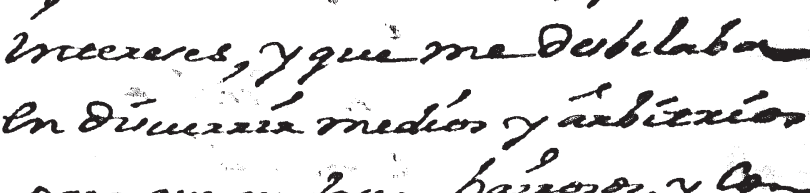
paxa gue gredaren haxeosoy y

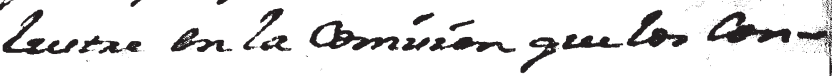
olseplo.

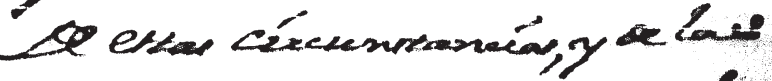

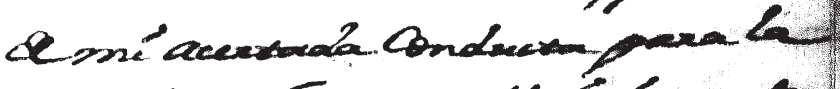

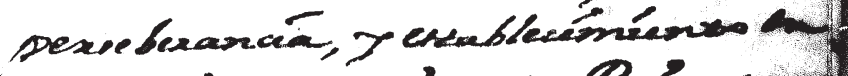

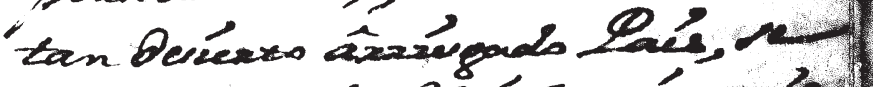

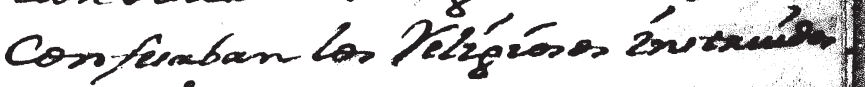

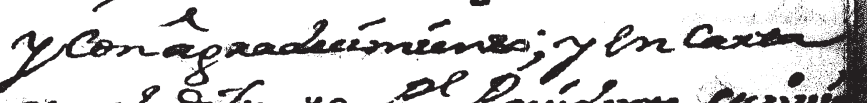

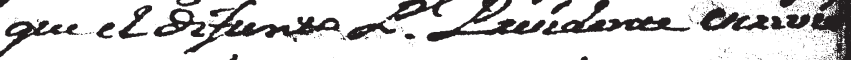

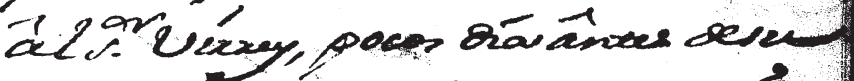

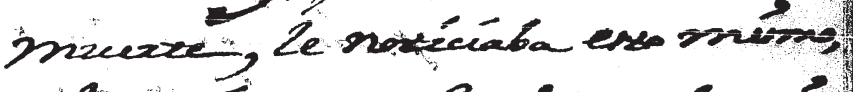

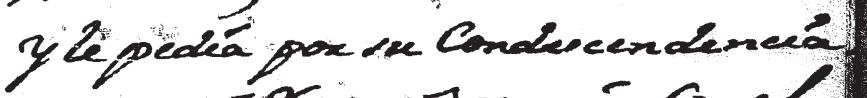

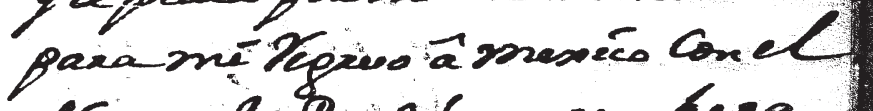

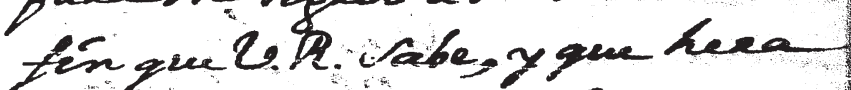
bodo Dediéad äl floxíno eszonum 
[fol. 3r]

en el destruido puesto, por gusto

mio, ni por disposiciones de la

âuthoridad que me incumbe.

II Sabidas $\left({ }^{\wedge} \mathrm{so}\right) \mathrm{n}$ las diligencias que

practique para que los religiosos

viviesen cerca de este Presidio, y

son notorias to $(\wedge d)$ as las âtenciones

mias con sus Personas, y con sus

intereses, y que me desbelaba

en discurrir medios y ârbitrios

10

para que quedasen hairosos, y con

lustre en la comision que los con-

dujo.

II De estas circunstancias, y de las

de mi acertada conducta para la

perseberancia, y establecimiento en

tan desierto ârriesgado Pais, se

confesaban los Religiosos instruidos

y con âgradecimiento; y en carta

que el difunto Padre Presidente escrivio

âl señor Virrey, pocos dias ântes de su

muerte, le noticiaba esto mismo,

y le pedia por su condescendencia

para mi regreso â mexico com el

fin que Vuestra Reverencia sabe, y que hera

todo dedicado âl Glorioso estatuto 
Spanish Colonial Documents Pertaining to Mission San Sabá

Yolnegio elo Hedivíduos alos Apossiviéos Collegión de la crus. ytan fixnands: $y$ era raxifa

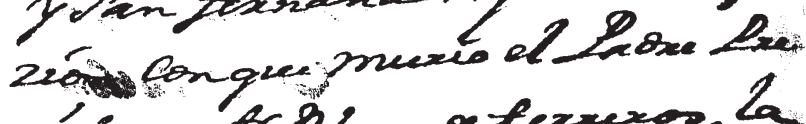

5 réderce Pr Blono deterwevos, la griéren spinker tembién los Sonselos míos, maneferemondose

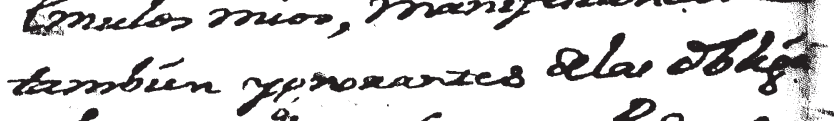

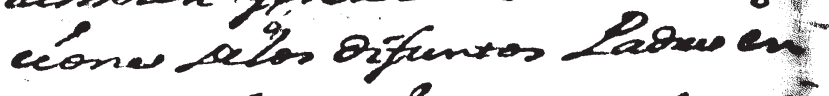
este Derénon y las que yes zeng

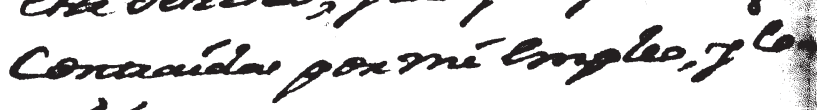
miñones.

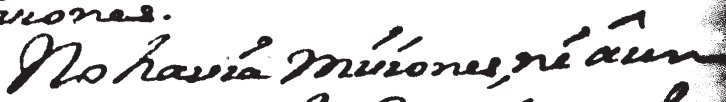

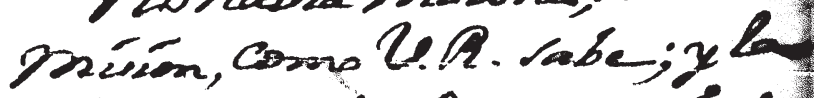

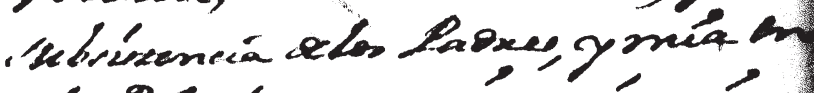

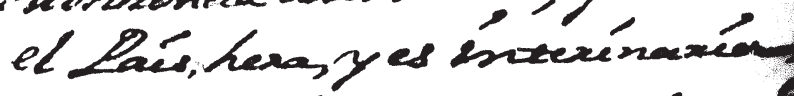
con Pépecus allo preespuad, yh Oxderes que re esperaban; 7 is tods esso por Delavere, tenéa Oh nada la greacer parte ala do

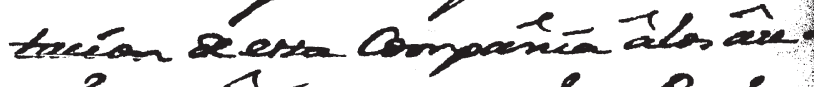
soilés y ârízenáa alos Ledrel

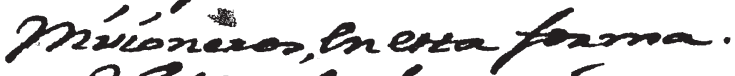

Hable dila hora eirimuneegy

25

tubelanoizéa quehaví Indés Desconacídos An la haviémecéón sel.

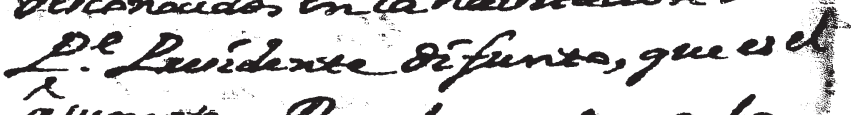
asumpo. Lara la escose ol los

118 


\section{[fol. 3v]}

y Reglas de los Individuos delos

Apostolicos Collegios de la Cruz

y San fernando: y esta satisfa-

cion, con que murio el Padre Pre-

sidente fray Alonso deterreros, la

quieren sepultar tambien los

emulos mios, manifestandose

tambien ygnorantes delas obliga

ciones delos difuntos Padres en

este destino, y las que yo tengo

contraidas por mi empleo, y co-

misiones.

II No havia misiones, ni âun

mision, como Vuestra Reverencia sabe; y la

subsistencia de los Padres, y mia en

el Pais, hera, y es interinaria

con respecto âlo preceptuado, y los

ôrdenes que se esperaban; y con

todo esto por delante, tenia desti-

nada la quarta parte de la do-

tacion de esta Compañia âlos âu-

xilios y âsistencia de los Padres

Misioneros, en esta forma.

II Hablo dela hora e instante que tube la noticia que havia Yndios

desconocidos en la havitacion del

Padre Presidente difunto, que es el âsumpto.

II Para la escolta de los 
Spanish Colonial Documents Pertaining to Mission San Sabá

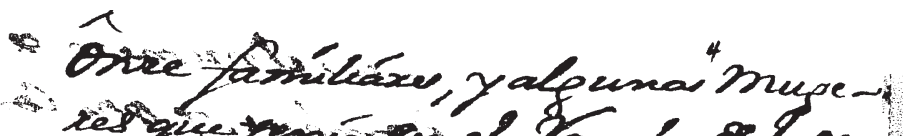

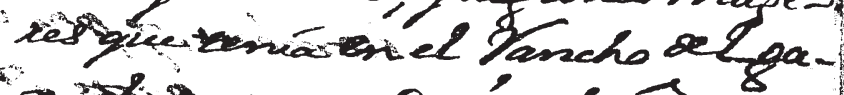

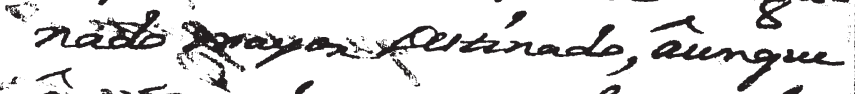
"Áprivnombre, para efears dela

5

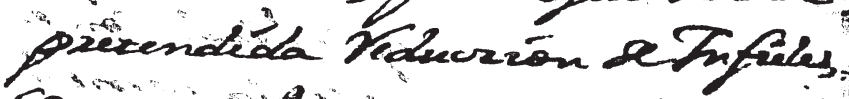

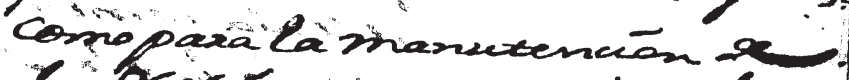

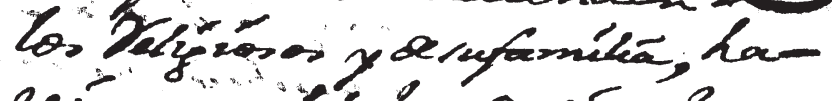

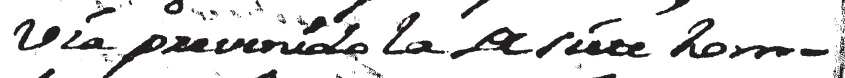

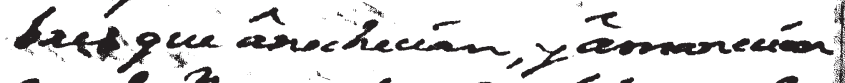

10 enel Homades Coníderands La oritivicén decíncs laguas en

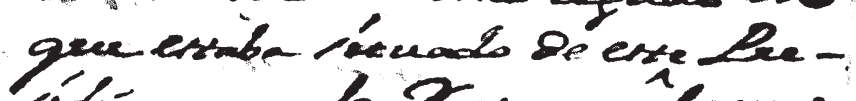

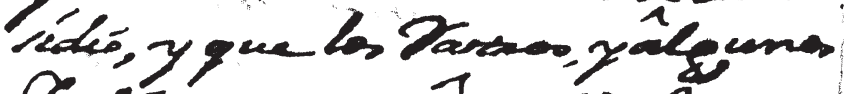

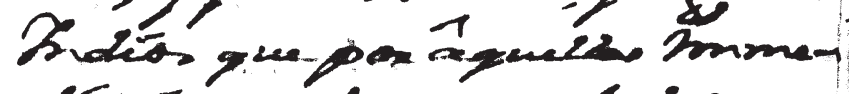
15

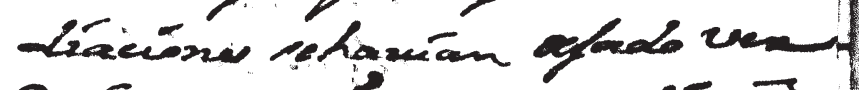

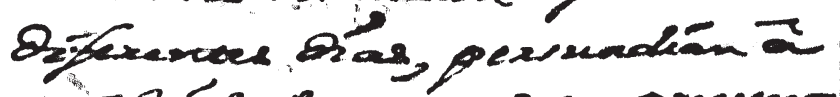

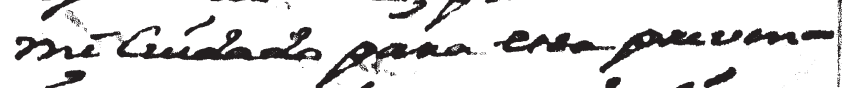
céon, qua zube no mucho terémpo

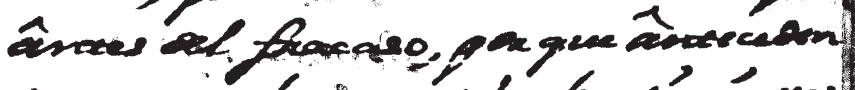

20

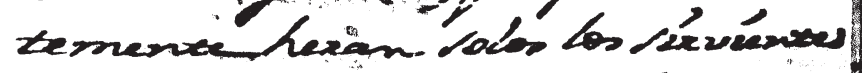

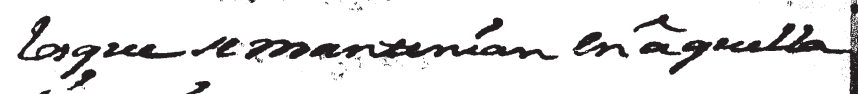
stecacéom - vo

oformarece estaban en Greadónsec.

25

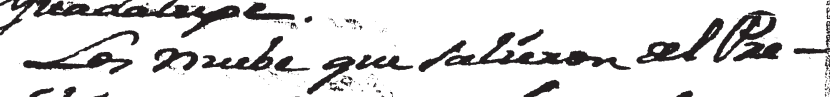

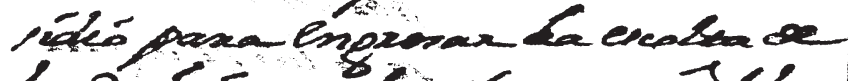

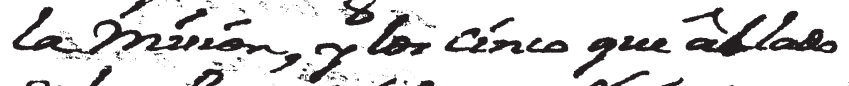

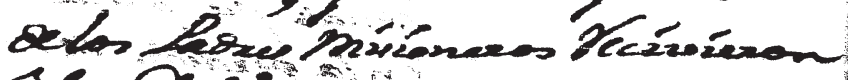
alt Ardeos gue lompenen ande

120 
[fol. 4r]

ônze familiares, y algunas mugeres que tenia en el Rancho del ganado mayor destinado, âunque âmi nombre, para efectos de la pretendida Reduccion de Ynfieles, como para la manutencion de los Religiosos y de sufamilia, havia prevenido la de siete hombres que ânochecian, y âmanecian en el Armados, considerando

la distancia de cinco leguas en que estaba situado de este Presidio, y que los Rastros, y âlgunos Yndios que por âquellas inmediaciones se havian dejado ver diferentes dias, persuadian â mi cuidado para esta prevencion, que tube no mucho tiempo ântes del fracaso, por que ântecedentemente heran solos los sirvientes los que se mantenian en âquella situacion.

II Los quatro que estaban en Guadalupe.

II Los nuebe que salieron del Presidio para engrosar la escolta de la Mision, y los cinco que âllado delos Padres Misioneros Recivieron âlos Yndios, que componen entre 
Spanish Colonial Documents Pertaining to Mission San Sabá

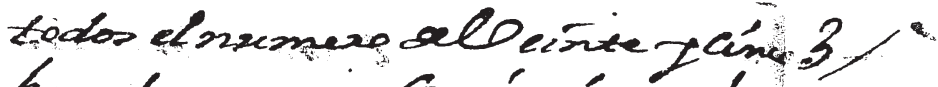
hombres, 7 por Conćfurénte la

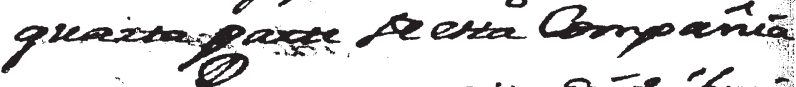

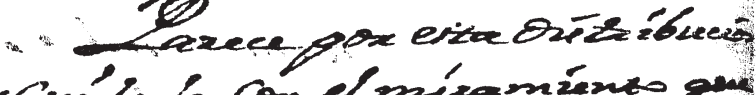

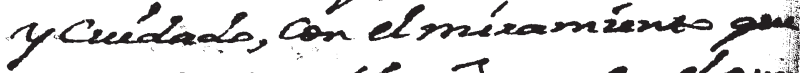
letenia tarnbén äque en elpen

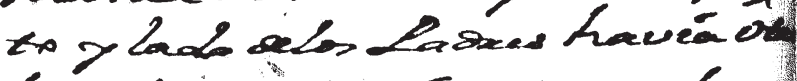

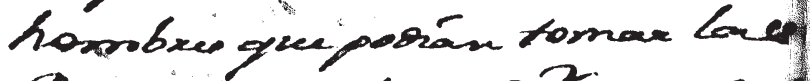

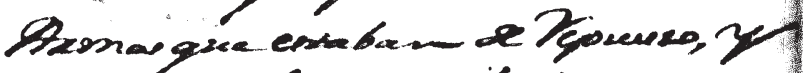

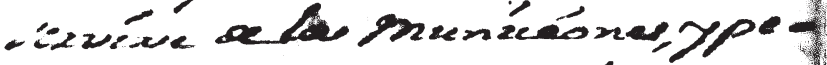

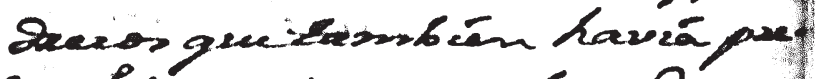
veneds; quie no estaban Daven

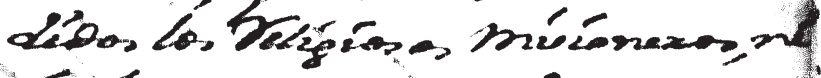

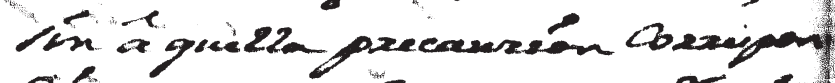

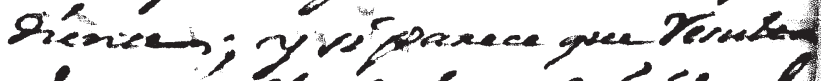

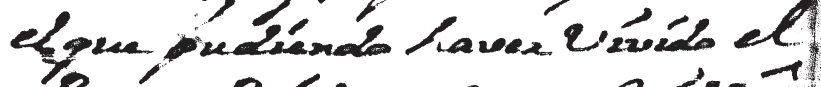

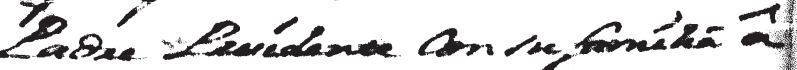

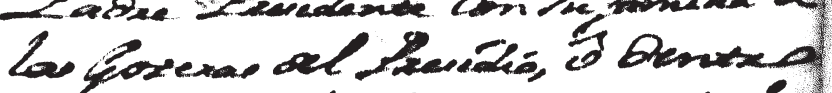

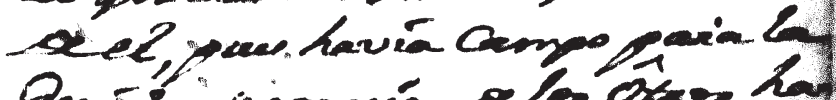
Devét "igparacén se los otser ha

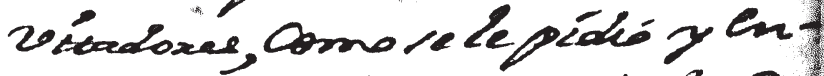

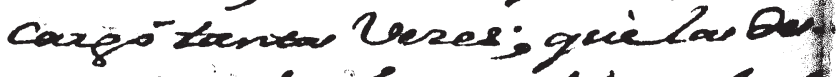
pracéa Kkbcedélas, subcexon hanel

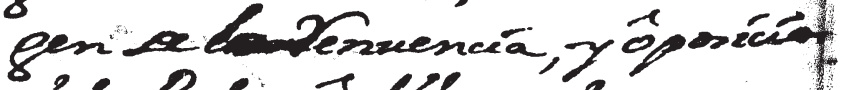
del Laxier, à bbew -2s;yquel pox esta Dason Deven Compadeed

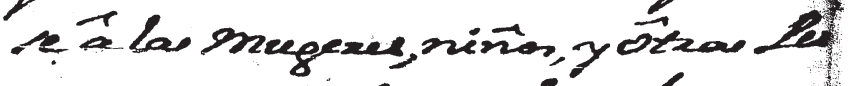
conar que padeen el geabeanny

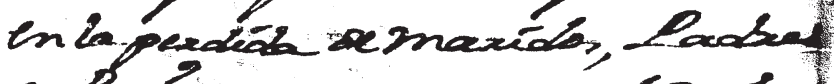

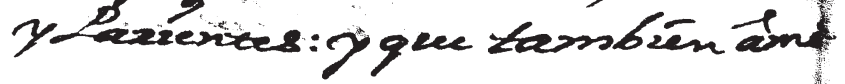


[fol. 4v]

\{left margin 3

todos el numero deVeinte y cinco

hombres, y por consiguiente la

quarta parte de esta Compañia

II Parece que esta distribucion

y cuidado, con el miramiento que

se tenia tambien â que en el pues-

to y lado delos Padres havia ôtros

hombres que podian tomar las

Armas que estaban de repuesto, y

servirse de las municiones, y pe-

dreros que tambien havia pre-

venidos; que no estaban desaten-

didos los Religiosos misioneros, ni

sin â quella precaucion correspon-

diente; y si parece que Resulta

el que pudiendo haver vivido el

Padre Presidente con su familia â

las Goteras del Presidio, ô dentro

de el, pues havia campo para la

devida separacion de los ôtros ha-

vitadores, como se le pidió y en-

cargó tantas veces; què las des-

gracias subcedidas, tubieran hori-

gen de (^la) renuencia, y ôposicion

del Padre, â libertarlas; y que

por esta razon deven compadecer-

se â las mugeres, niños, y ôtras Per-

sonas que padecen el quebranto

en la perdida de maridos, Padres

y Parientes: y que tambien âmi 
Spanish Colonial Documents Pertaining to Mission San Sabá

Cenedebe \& Dowricia la aztepcéox, y el míxanzéxto pox los invereites gree heperdíds, ypox Razexima soprewes àla Censera

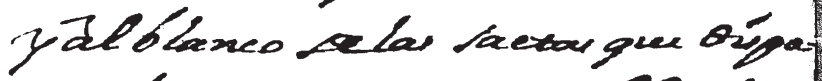
ran los ygrpxanxes, gree Tepularmente por sosas en el pruncto,

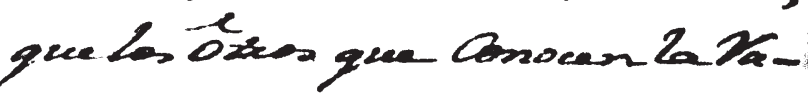
zon.

10

Q 1 Cox exe nombibe, y otwer muchos by arvehedox âlou buenas

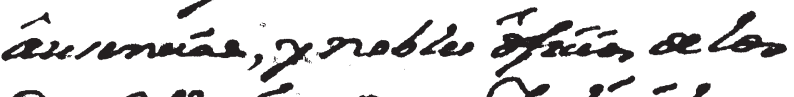

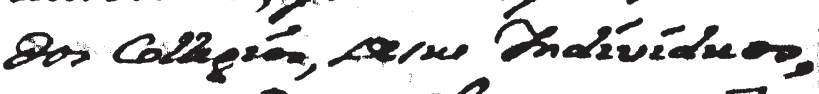

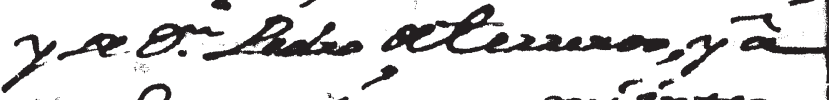

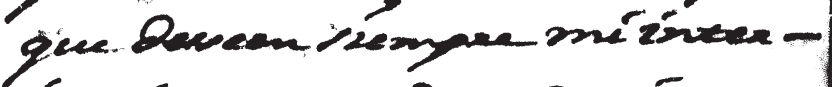

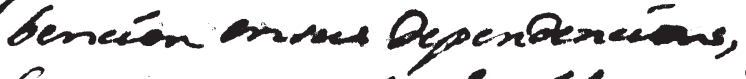

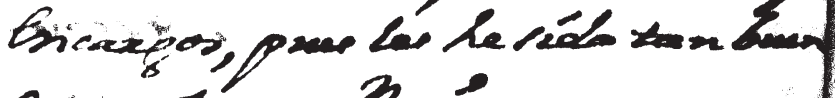

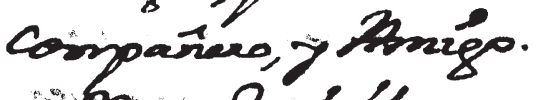

- Oro en Dedoble guep pende bahi-

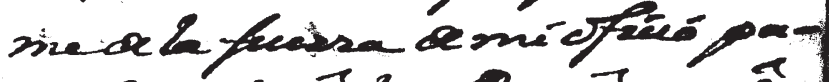

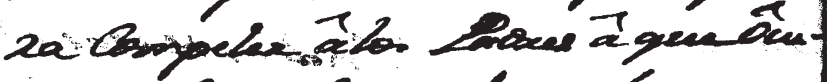
pien Dow figpax, Una ber gene

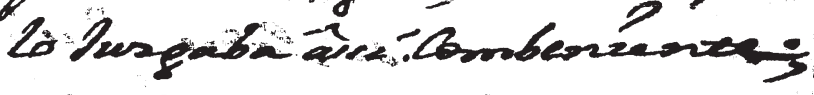
pein terripenes 2Dudary

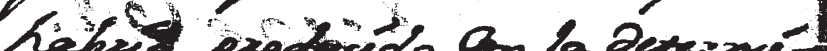

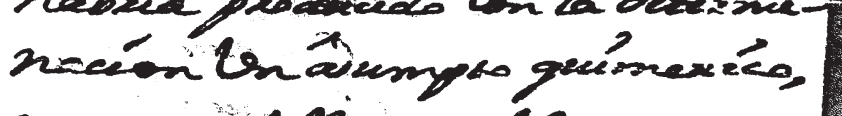

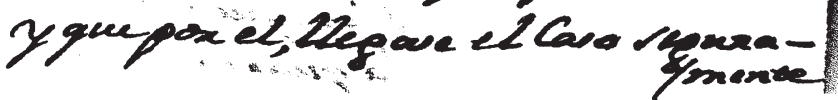




\section{[fol. 5r]}

se me debe de xusticia la âtencion, y el miramiento por los intereses que he perdido, y por haverme expuesto â la censura y âl blanco de las saetas que dispa-

ran los ygnorantes, que regularmente son mas en el mundo, que los ôtros que conocen la razon.

II Por este motibo, y ôtros muchos soy acrehedor âlas buenas âusencias, y nobles ôficios de los dos Collegios, de sus Yndividuos, y de Don Pedro deterreros, y â que desseen siempre mi inter-

bencion en sus dependenci(^a)s, y encargos, que les he sido tan buen compañero, y Amigo.

II No es dudable que pude balerme de la fuerza de mi oficio pa-

ra compelar â los Padres â que ôcupasen ôtro lugar, una bez que lo juzgaba âssi. combeniente; pero tampoco es de dudar que habria producido con la determi-

nacion un âsumpto quimerico, y que por el, llegase el caso seguramente 


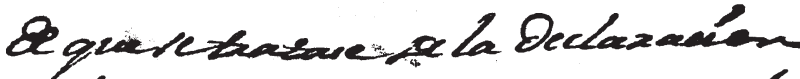

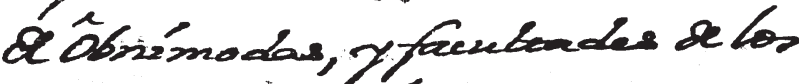

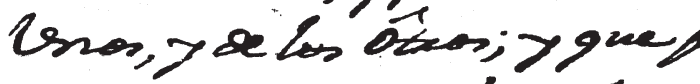

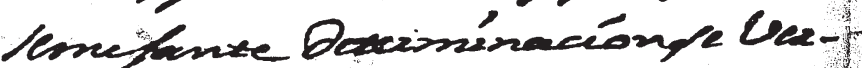
wen, y Concurxiéren ifferencias,

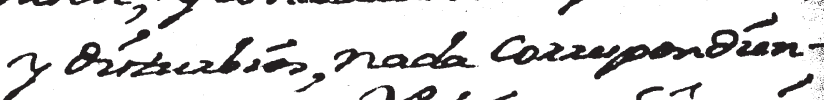

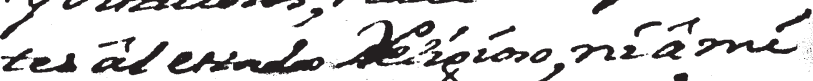
Deves ala $\mathrm{Pan}_{2}$, dela resizon:per

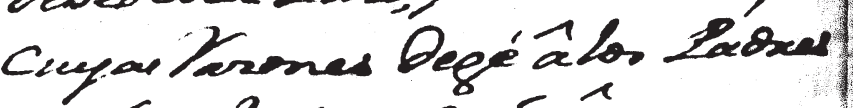

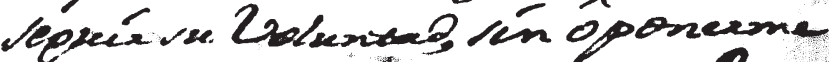
8

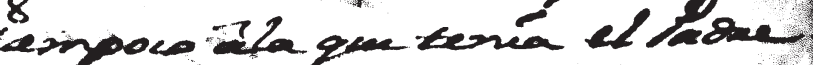
Pexplinax in tembear geentes,

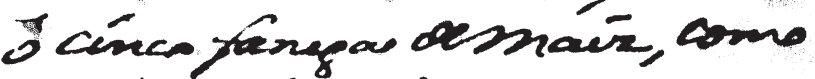

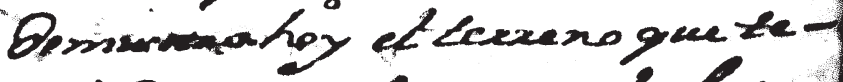

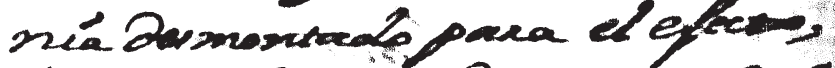
anves de haves Judies en Príl

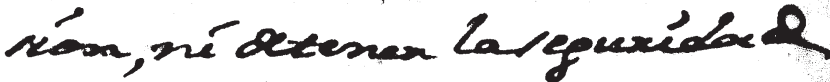
Epermancer âgur por cuy mosibomeperruadb ägue te Le Reríriérían bos anbios delos

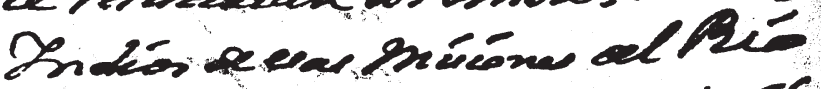

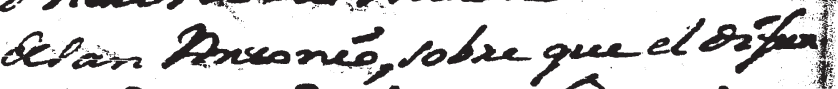

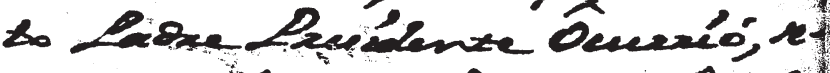
gun on has se scho, à la âwho 2íla? sel?

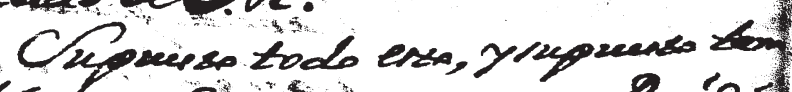

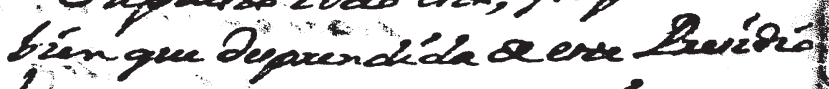
La gieaven pave depedoracion en 


\section{[fol. 5v]}

de que se tratase de la declaracion de ôbnimodas, y facultades de los Unos, y de los ôtros; y que para semejante determinacion $\left({ }^{\wedge} \mathrm{s}\right)$ e versasen, y concurriesen diferencias, y disturbios, nada correspondientes âl estado (^Re)ligioso, ni â mi desseo dela Paz, y dela Union: por cuyas Razones degé â los Padres seguir su Voluntad, sin ôponerme tampoco âla que tenia el Padre Presidente en sembrar quatro ô cinco fanegas de Maiz, como demuenstra hoy el terreno que tenia desmontado para el efecto, ântes de haver Yndios en mision, ni detener la seguridad de permanecer âqui; por cuyo motibo me persuado â que se le Resistirian los embios de los

Yndios de esas Misiones del Rio deSan Antonio, sobre que el difunto Padre Presidente ôcurrió, se gun me havia dicho, â la âuthoridad de Vuestra Reverencia.

II Supuesto todo esto, y supuesto tambien que desprendida de este Presidio la quarta parte desu dotacion en 


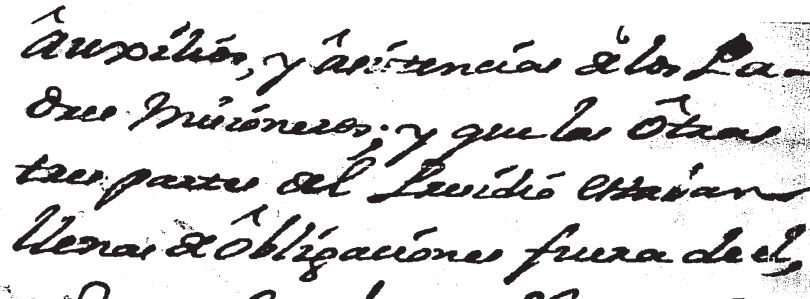

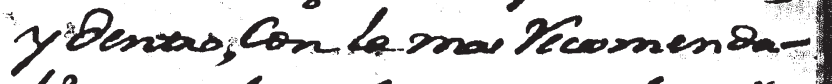

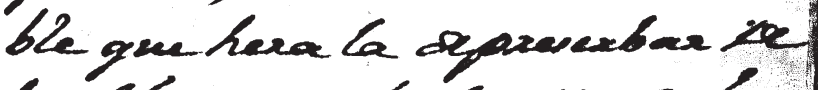
La Zziancá y baxbaxicho seb.

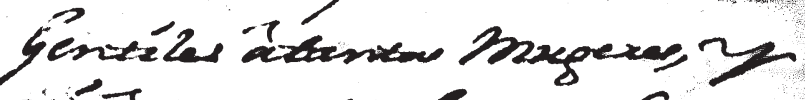

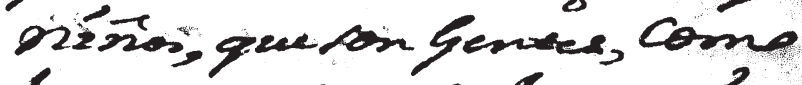

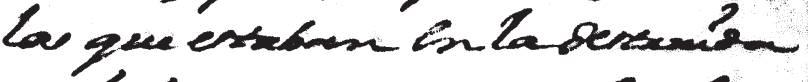

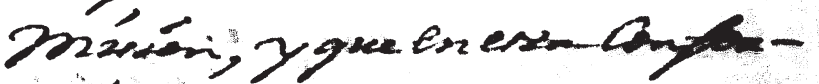

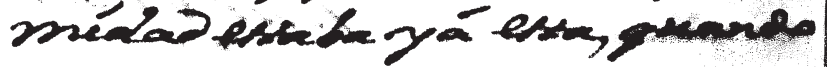

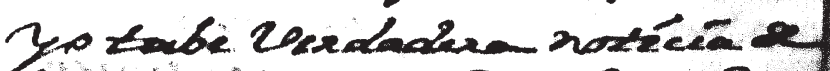

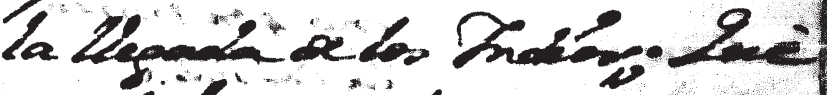
puati haver pet mas paren

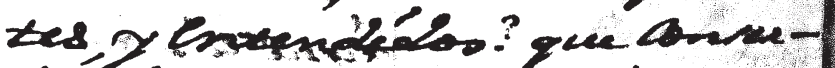

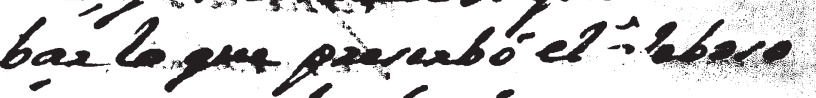

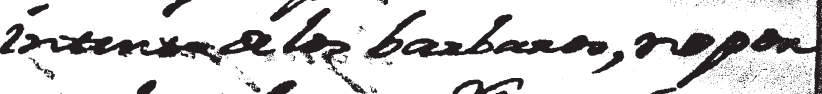

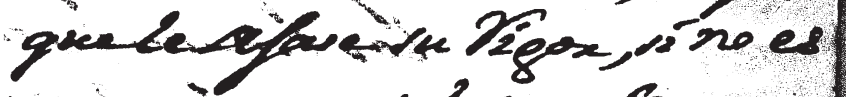

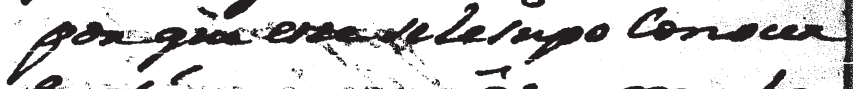

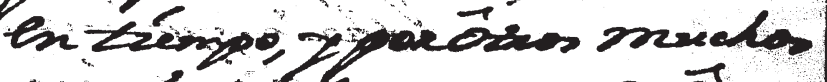

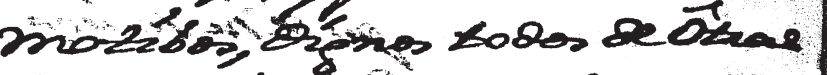

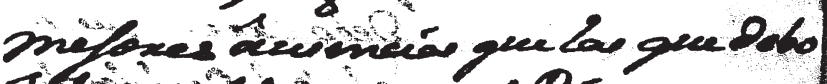

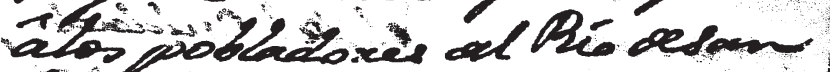
Brivine, guxenes sjexeran of crexos hubivian hitenakels que la 
[fol. 6r]

\{top left margin\} 6

âuxilios, y âsistencias de los Padres Misióneros; y que las ôtras tres partes del Presidio estaban llenas de ôbligaciones fuera de el, y dentro, con la mas recomenda-

ble que hera la depreserbar de la tirania y barbaridad delos Gentiles âtantas mugeres, $\mathrm{y}$ niños, que son Gentes, como las que estaban en la destruida Mision, y que en esta conformidad estaba yá esta, quando yo tube verdadera noticia de la llegada de los Yndios; Què pude haver echo mas para merecer el aplauso delos prudentes, y entendidos? que conserbar lo que preserbó el [â]leboso intento de los barbaros, no por que le dejase su Rigor, si no es por que este se le supo conocer en tiempo, y por ôtros muchos motibos, dignos todos de ôtras mejores âusencias que las que debo âlos pobladores del Rio deSan Antonio, quienes si fueran discretos tubieran entendido que la 
Spanish Colonial Documents Pertaining to Mission San Sabá

fomma guede hace malgraxy I nucusteos mar bien Concertado. Derǵgnés, pexene podra guím nos la Glaxia se haver óbrads

5 gun las Meglas sela prudencia: Y $\checkmark$ barka yeu len hombre, no haya obmízíds Cora alguma Bnducen te à Conregrecí las empxesas; pren. Los buenos, ômalos logees no Dh

10

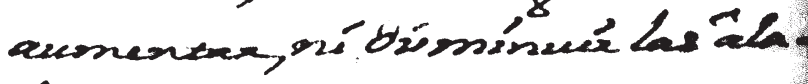
Banzas.

Plsé píenan lon gue laben mm que $y 0, y$. 20 have sémpere lo mon

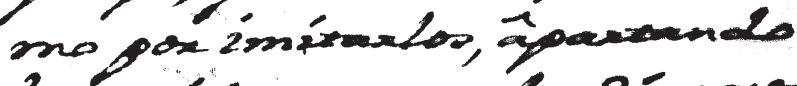
15 la comílexacsion selos oŕcuen constaviéos.

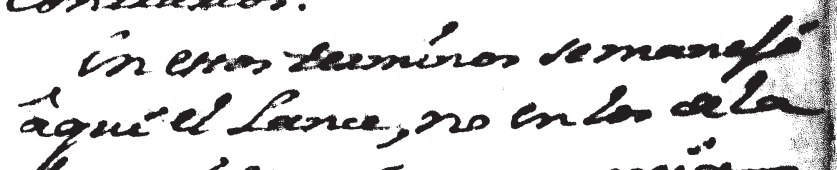

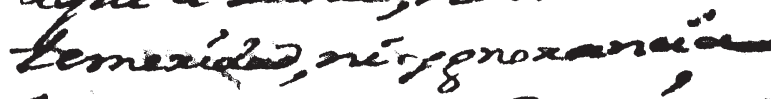

20 ague previe gace an los ensivelos en era oridunción proponsénal antofaciros me hodos yos zecpénnes, en eltrém.

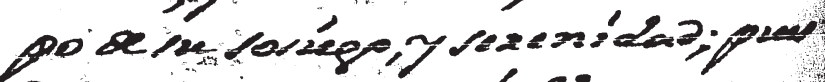
25 Deba crees gue it 1 hallaran en

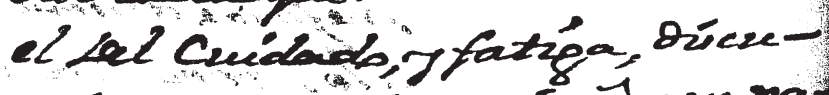

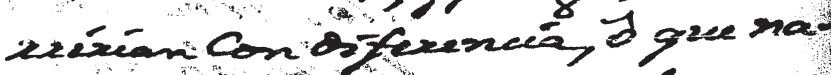
da oricuresíán, que reséa 6 mas

130 
[fol. 6v]

\{top left margin\} 9

fortuna queda hacer malograr

nuestros mas bien concertados

designios, pero no podra quitar-

nos la Gloria de haver ôbrado se-

gun las Reglas dela prudencia: y

basta que un hombre, no haya

obmitido cosa alguna conducen-

te â conseguir las empresas; pues

los buenos, ô malos logros no deben

aumentar, ni disminuir las âla-

banzas.

II Assi piensan los que saben mas

que yo, y io hare siempre lo mis-

mo por imitarlos, âpartando

la consideracion delos discursos

contrarios.

II En estos terminos se manejó

âqui el Lance, no en los de la

temeridad, ni ygnorancia

âque parece que conspiran

los emulos en esa distancia

proponiendo antojadizos me-

thodos, y direcciones, en el tiem-

po de su sosiego, y serenidad; pues

debo creer que si se hallasen en

el del cuidado, y fatiga, discu-

rririan con diferencia, ô que na-

da discurririan, que seria lo mas 
Spanish Colonial Documents Pertaining to Mission San Sabá

ciesto.

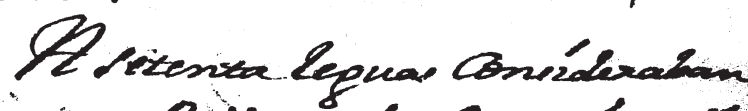

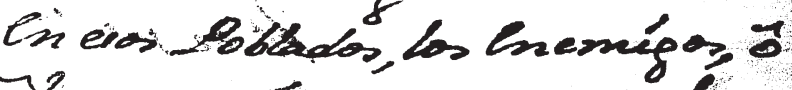

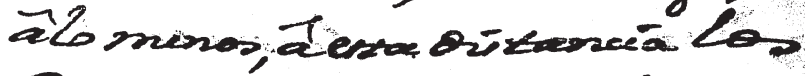
Devián Puspars, ptubiéen C-

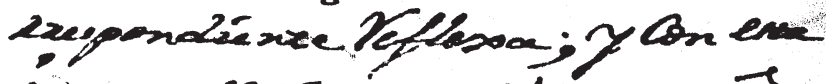

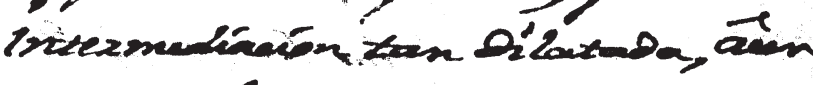

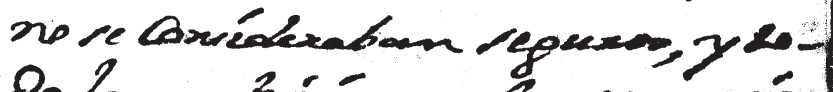

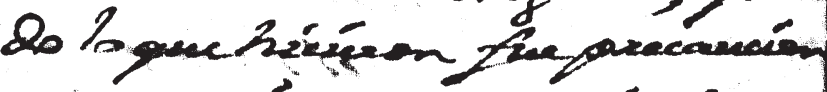

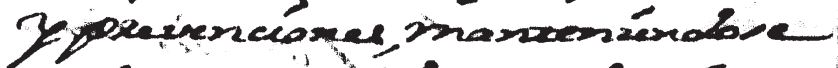

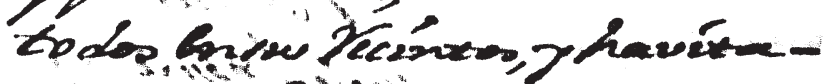

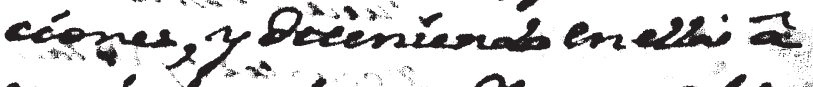

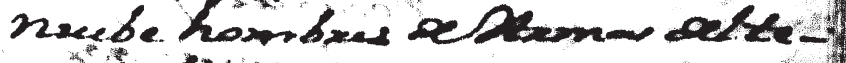

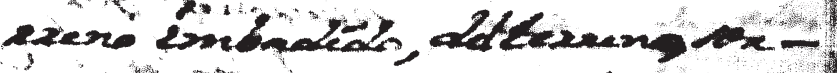

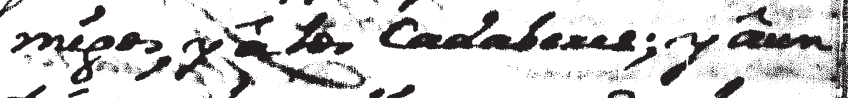

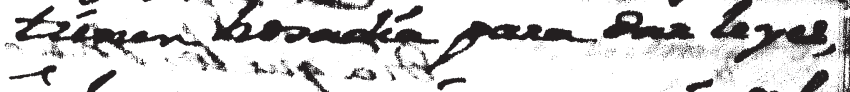

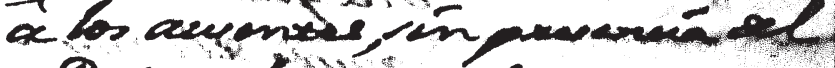

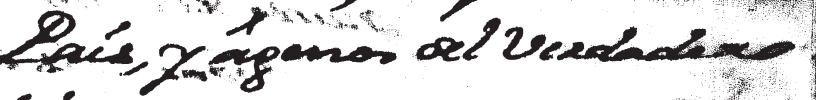
lebage.

- Ept alo gue para encer pik-

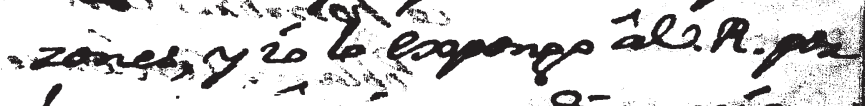

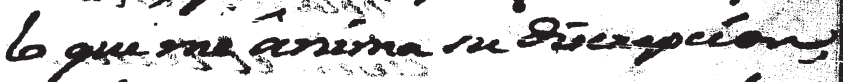

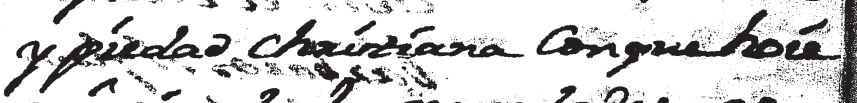

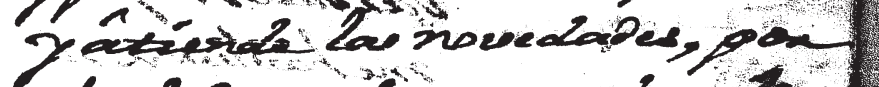

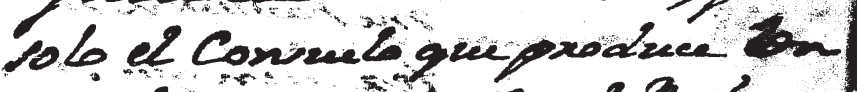
cerabeo deanimo anel Ancisos 


\section{[fol. 7r]}

cierto.

II A setenta leguas consideraban en esos Poblados, los enemigos, ô âlo menos, â esta distancia los devian juzgar, si tubiesen correspondiente Reflexa; y con esta intermediacion tan dilatada, âun no se consideraban seguros, y todo lo que hicieron fue precaucion y prevenciones, manteniendose todos en sus Recintos, y havitaciones, y deteniendo en ellas â nuebe hombres de Armas del terreno imbadido, del terren $\left({ }^{\wedge} 0\right)$ sorprehendido, y el que vio âlos enemigos, y â los cadaberes; y âun tienen hosadia para dar leyes â los ausentes, sin presencia del Pais, y âgenos del verdadero subceso.

II Esto es lo que pasa en esas poblazones, y io lo expongo â Vuestra Reverencia por lo que me ânima su discrepcion, y piedad christiana con que hoie y âtiende las novedades, por solo el consuelo que produce $\left({ }^{\wedge} \mathrm{u}\right) \mathrm{n}$ desahogo deanimo con el Amigo, 
Spanish Colonial Documents Pertaining to Mission San Sabá

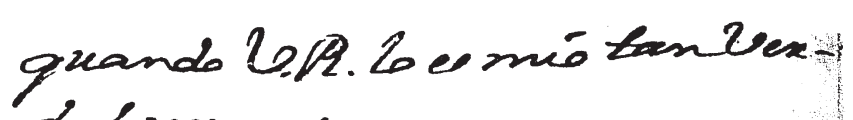
daderamente.

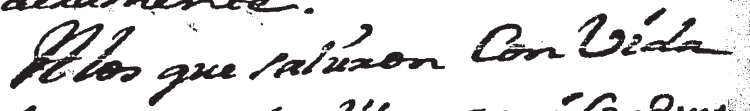

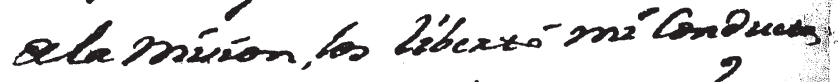

5

yno los Jiseos motibes que rpisuan.

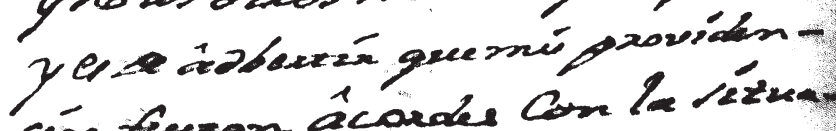

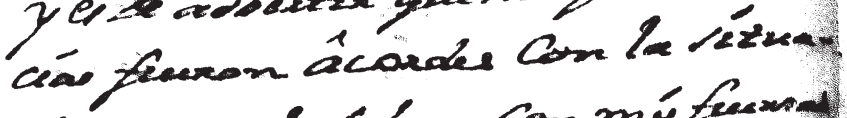
cíon, con el Lubreo, con mófresont

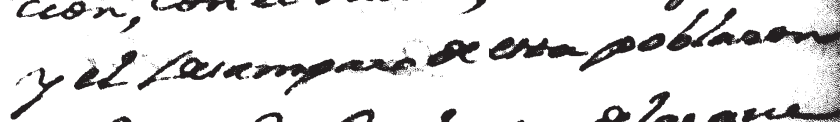

10

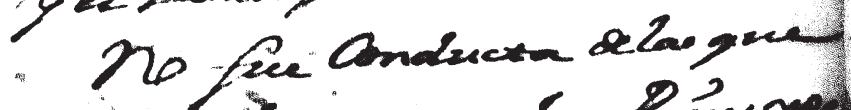

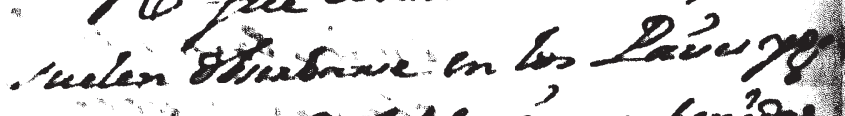
noxaries, y butiéles ámparbenion

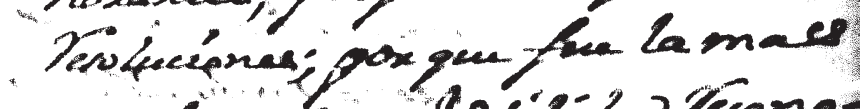

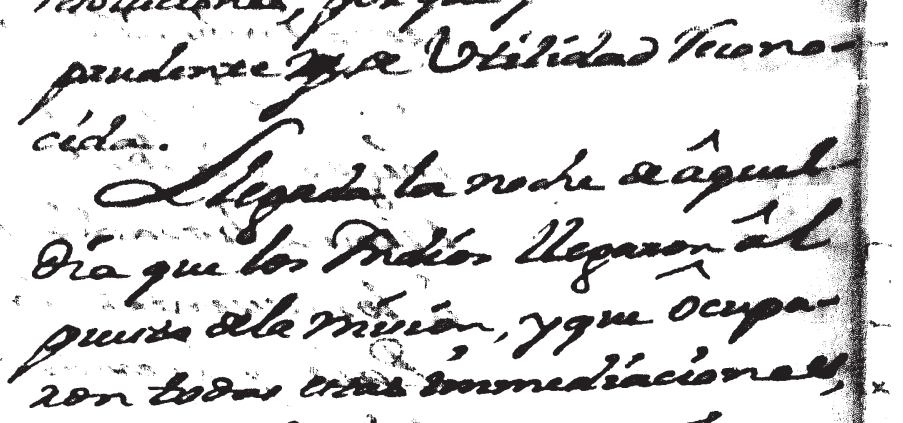
20

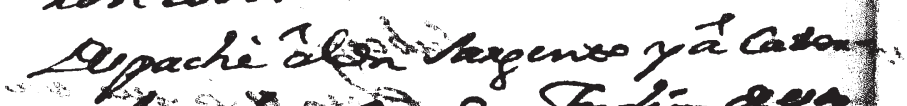

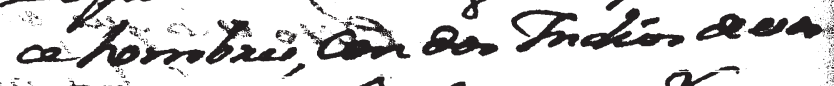

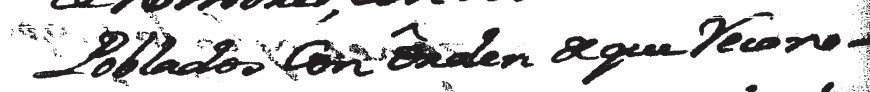

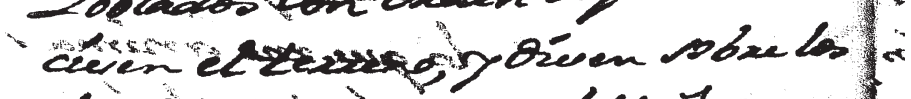

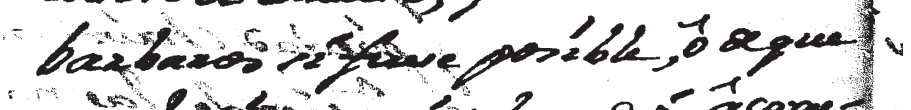
25

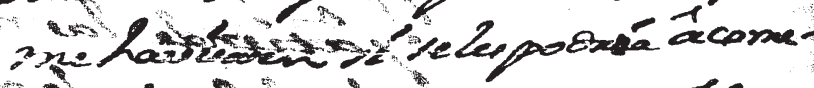

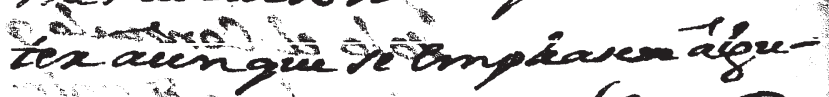
(na)

134 


\section{[fol. 7v]}

quando Vuestra Reverencia lo es mio tan ver-

daderamente.

\section{Alos que salieron Con Vida}

dela Mision, los libertó mi conducta,

y no los ôtros motibos que se piensan,

y es de âdbertir que mis providen-

cias fueron âcordes con la situa-

cion, con el subceso, con mis fuerzas

y el desamparo de esta poblazon

II No fue conducta de las que

suelen obserbarse en los Paises yg-

norantes, y defaciles imprebenidas

Resoluciones; por que fue la mas

prudente $\left({ }^{\wedge} \mathrm{y}\right)$ de Utilidad Recono-

cida.

II Llegada la noche de âquel

dia que los Yndios llegaron âl

puesto dela mision, y que ôcupa-

ron todas estas immediaciones,

despachè âUn sargento y â cator-

ce hombres, con dos Yndios de esos

Poblados con ôrden de que Recono-

ciesen el terreno, y diesen sobre los

barbaros si fuese posible, ô de que

me havisasen si se les podria âcome-

ter âun que se empleasen âlgu-

(na 


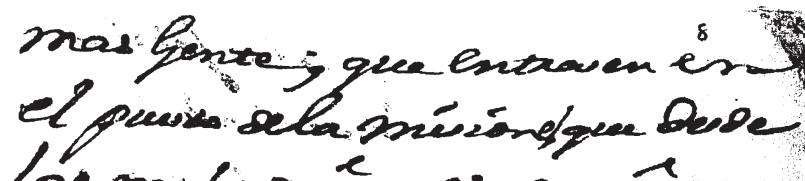
Lor $c$ a

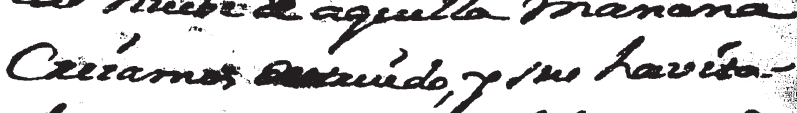

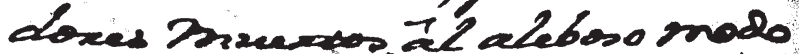

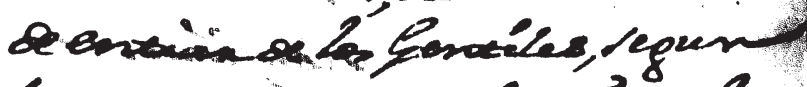

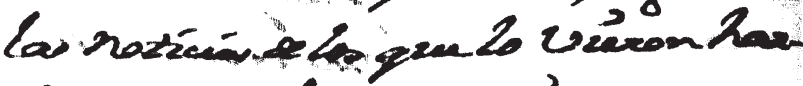

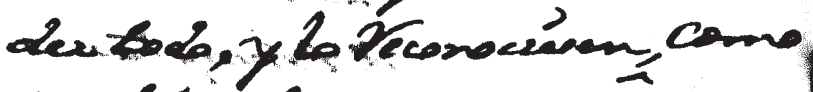

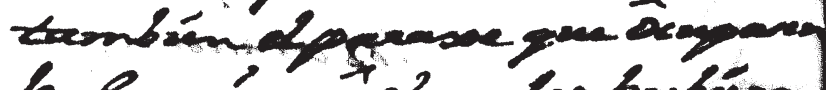

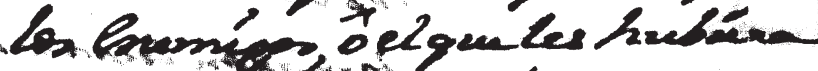

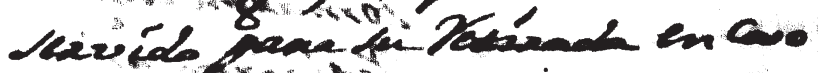

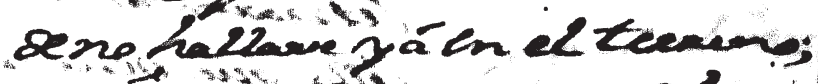

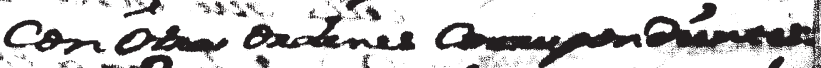

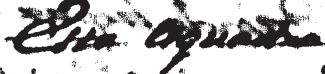

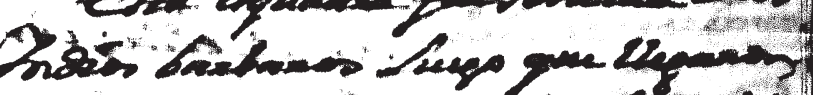

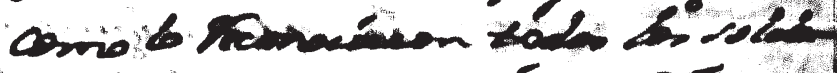

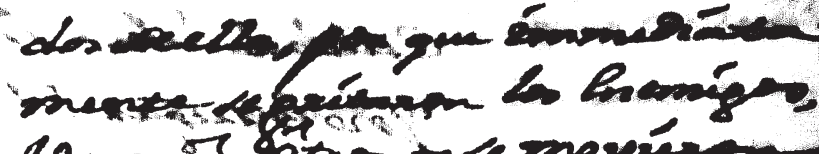

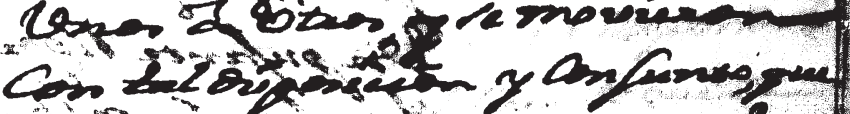

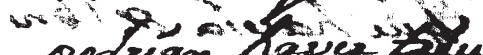

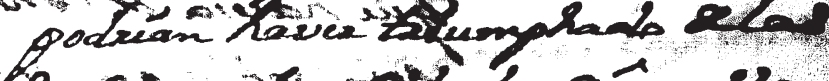

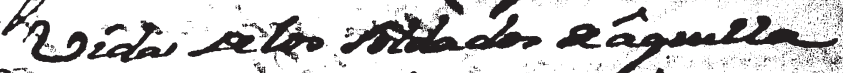

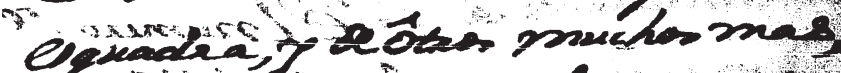

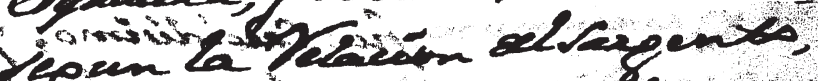
Sopen la 8

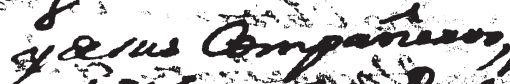

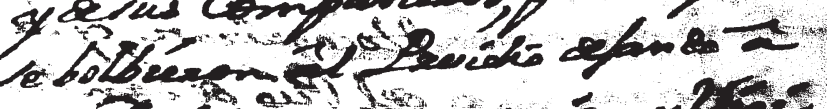
625

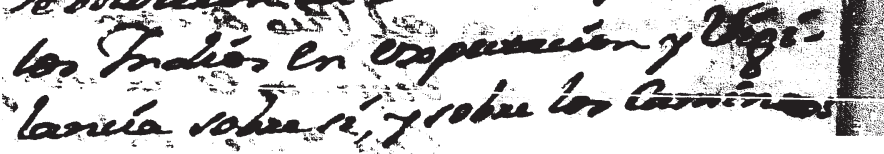


[fol. 8r]

mas Gente; que entrasen en

el puesto dela mision $\left({ }^{\wedge} \mathrm{e}\right)$ que desde

las nuebe de âquella mañana

creiamos destruido, y sus havita-

dores muertos, âl aleboso modo

de entrar de los Gentiles, segun

las noticias de los que lo vieron har-

der todo, y lo Reconociesen, como

tambien el paraxe que ôcupasen

los enemigos, ô el que les hubiera

servido para su Retirada en caso

de no hallarse yá en el terreno;

con ôtras ordenes correspondientes:

II Esta esquadra fue sentida delos

Yndios barbaros luego que llegaron

como lo Reconocieron todos los solda-

dos de ella, por que immediata-

mente se gritaron los enemigos,

Unos â ôtros, y se movieron

con tal disposicion y conjunto, que

podrian haver triumphado delas

Vidas de los soldados de âquella

esquadra, y de ôtros muchos mas,

segun la Relacion del sargento,

y de sus compañeros, por lo que

se bolbieron âl Presidio dejando â

los Yndios en expectacion y Vigi-

lancia sobre si, y sobre los Caminos 


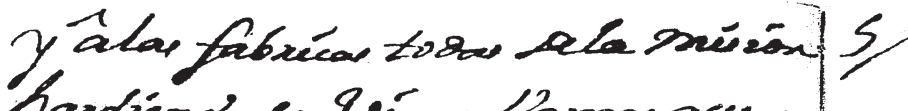

havdiénad en Rízas Lama geum aluesobiabure bien abuec paxavey Dóndios Gentile, Cuédads. cos yá pox el Pemox guec hrexty.

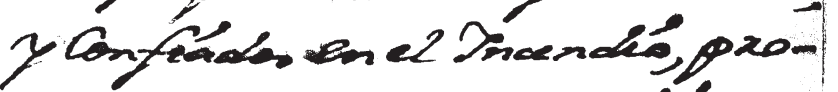
crevarom m Heperardo, y ol logen Lestumphar sher lab fenver

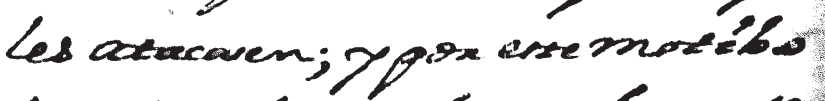

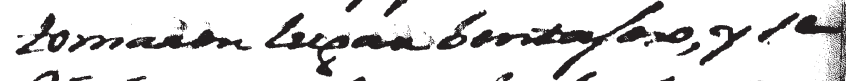

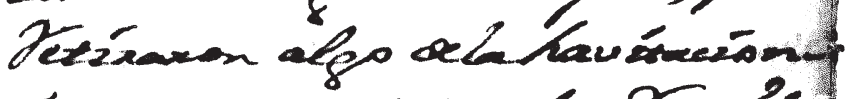

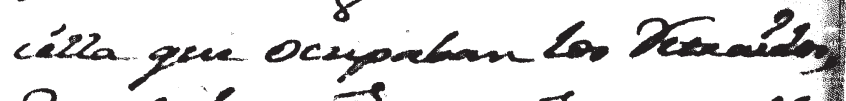

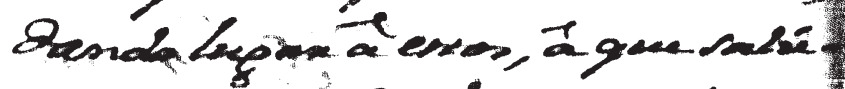

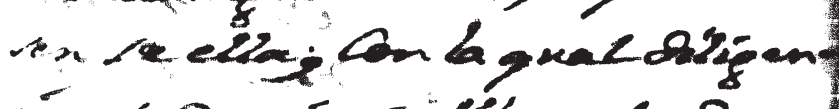

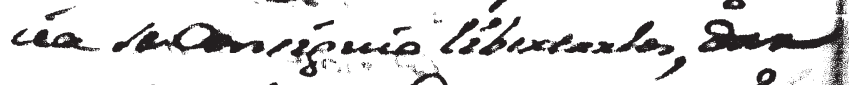

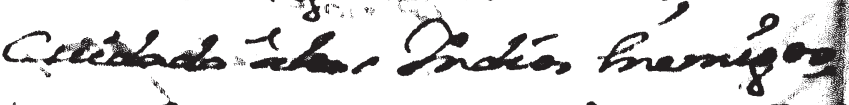

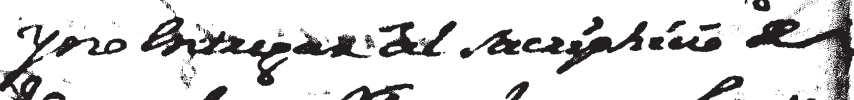

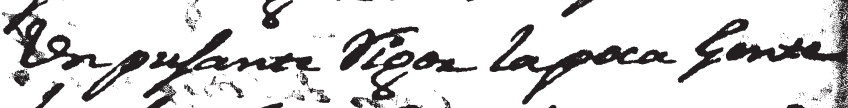

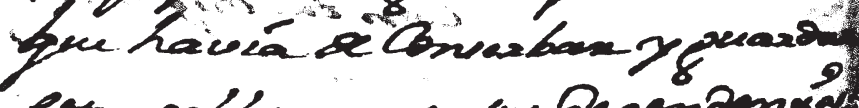

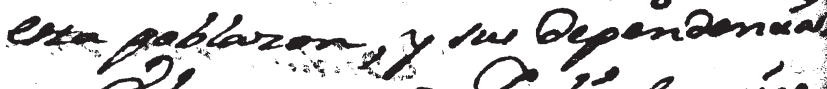
Q2 numero ar Bndés enemíg

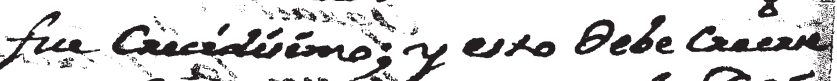

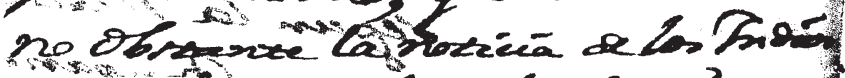

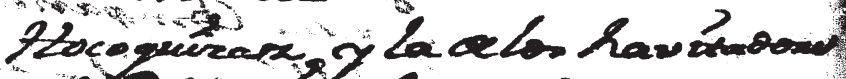

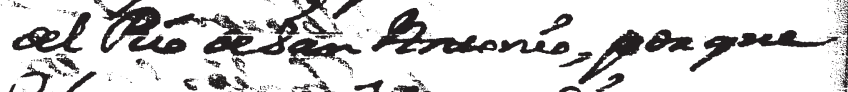

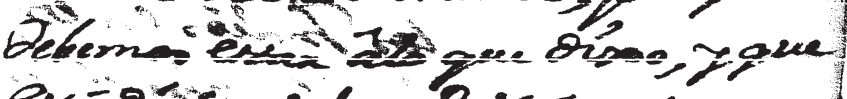

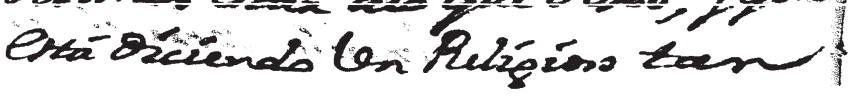


[fol. 8v]

\{top left margin\} 5

y âlas fabricas todas de la mision

hardiendo en Vivas llamas que

alumbraban bien aquel paraxe

II Los Yndios Gentiles, cuidado-

sos yá por el Rumor que hizieron

y confiados en el Yncendio, pro-

curaron su Resguardo, y el logro

de triumphar sobre las Gentes que

les atacasen; y por este motibo

tomaron lugar bentajoso, y se

Retiraron algo dela havitacion-

cilla que ocupaban los Retraidos,

dando lugar â estos, â que salie-

sen de ella; con la qual diligen-

cia se consiguio libertarlos, dar

cuidado âl(^os) Yndios enemigos, y no entregar âl sacriphicio de

un pujante Rigor la poca Gente

que havia de conserbar y guardar

esta poblazon, y sus dependencias.

II El numero de Yndios enemigos

fue crecidisimo; y esto debe creerse

no obstante la noticia de los Yndios

Hocoquicasz, y la de los havitadores

del Rio deSan Antonio, por que

debemos estar âlo que dixo, y que

está diciendo Un Religioso tan 
Spanish Colonial Documents Pertaining to Mission San Sabá

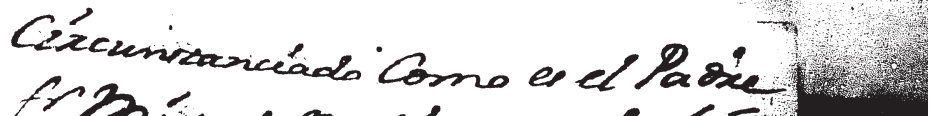

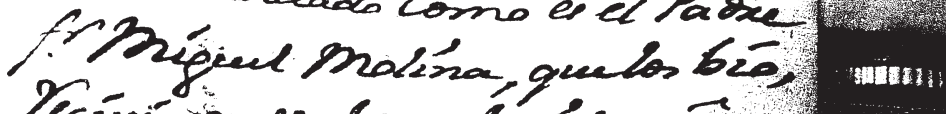
Tecéni de clos Cone heved yau. C. habbitambién, yáb, que

5 oreen Disess hombien guee expexénerisuran lo múnomo.

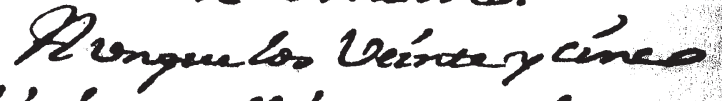

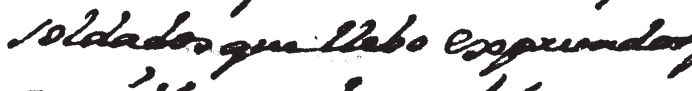

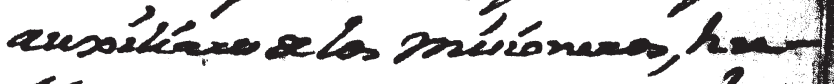

10 biécan de en el persocol-

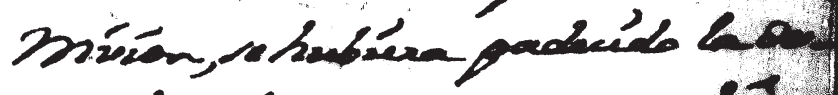

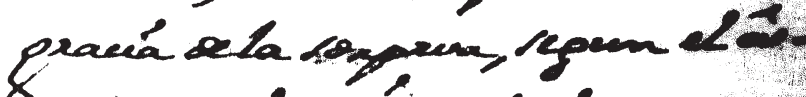
stease shade sén

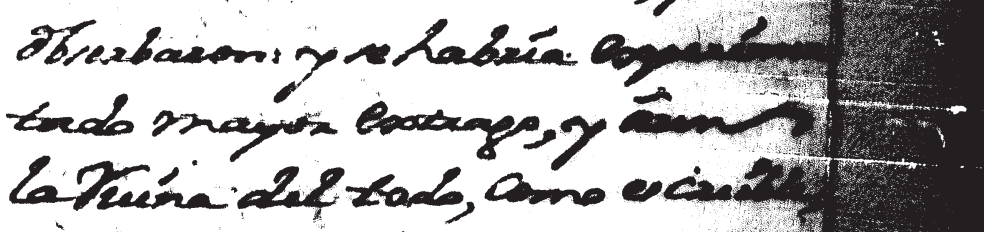

15

20

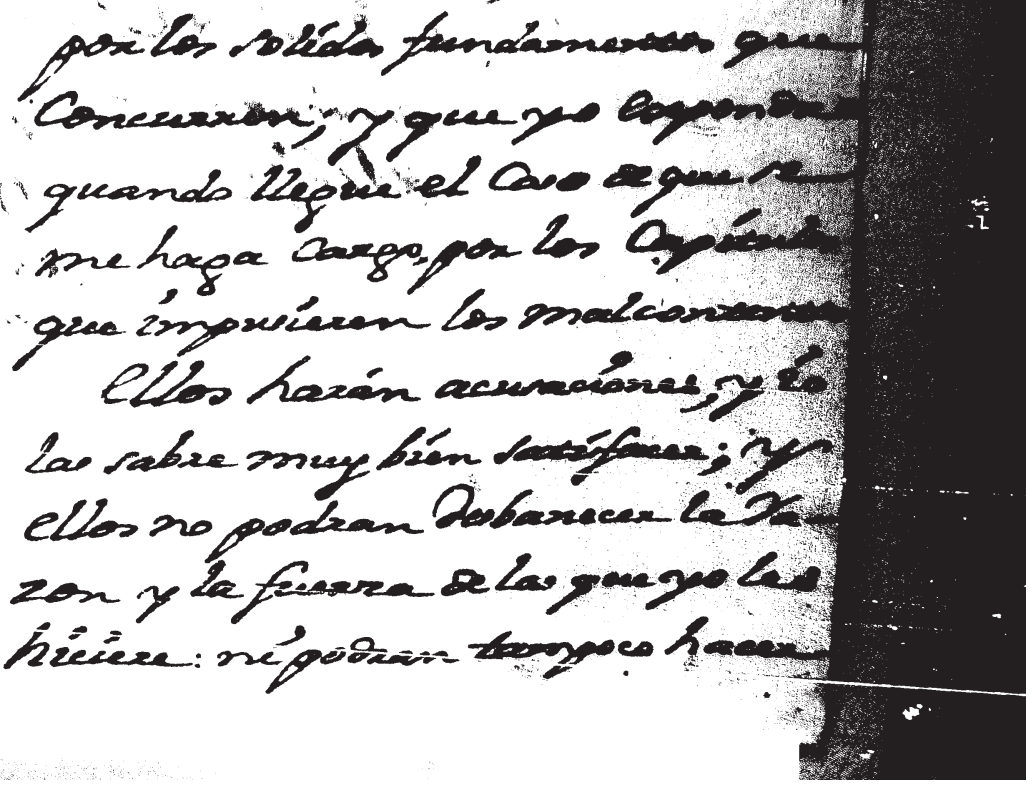

25

140 
[fol. 9r]

circunstanciado como es el Padre fray Miguel Molina, que los bio, recivio de ellos Una herida, y âun los habló tambien, y âlo que dicen ôtros hombres que expe-

rimentaron lo mismo.

II Aunque los Veinte y cinco

soldados que llebo expresados por auxiliares de los misioneros, hubieran estado en el puesto de la

Mision, se hubiera padecido la desgracia de la sorpresa, segun el âstuto modo de introducirse, que obserbaron: y se habria experimentado mayor extrago, y âun la Ruina del todo, como es creible, por los solidos fundamentos que concurren; y que yo expondre quando llegue el caso de que se me haga cargo, por los Capitulos que impusieren los malcontentos

II Ellos harán acusaciones, y ío las sabre muy bien satisfacer; $y$ ellos no podran desbanecer la Ra zon y la fuerza de las que yo les hiciere: ni podran tampoco hacer 
Spanish Colonial Documents Pertaining to Mission San Sabá

5

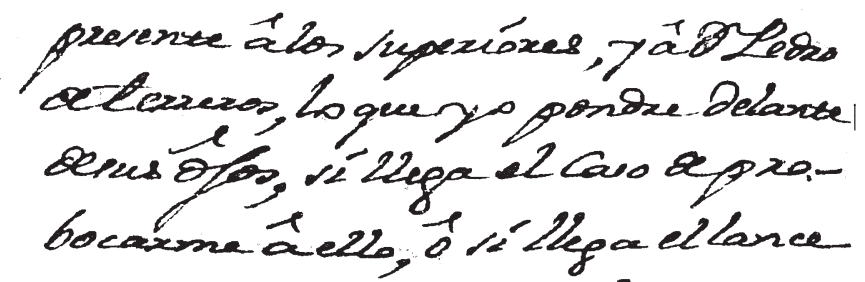

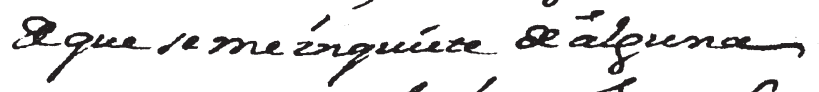
manexa; y no lo haxe âsotes, Como lo labre bode el munde,peed

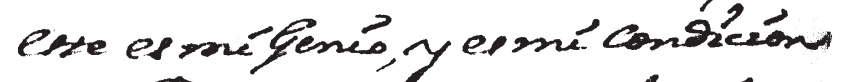

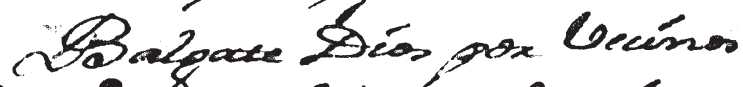

10

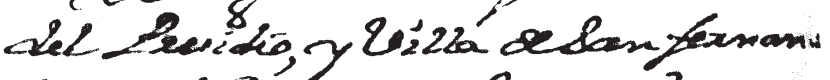
doncllís desan Busonisy. quesesto me han echo defendex En essa paca molester la atesectón

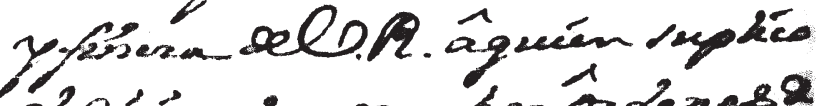

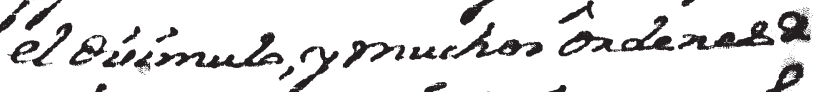
sel>58\%
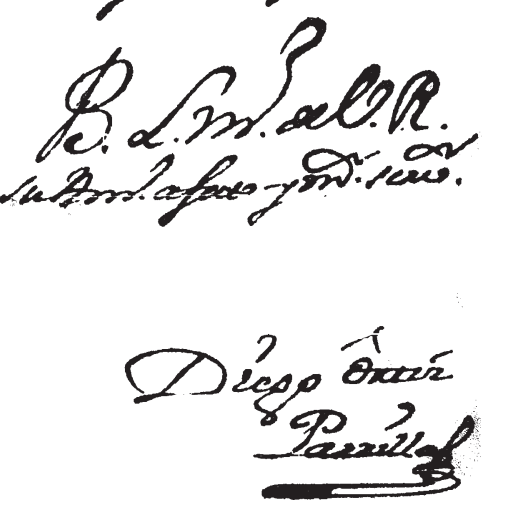


\section{[fol. 9v]}

presente â los superiores, y â Don Pedro

deterreros, lo que yo pondre delante

desus ôjos, si llega el caso de pro-

bocarme â ello, ô si llega el lance

de que se me inquiete de âlguna

manera; y no lo hare ântes, co-

mo lo sabrá todo el mundo, pues

este es mi Genio, y es mi condicion

II Balgate Dios por Vecinos

del Presidio, y Villa deSan fernan-

do en el Rio deSan Antonio, y

quanto me han echo difundir

en esta para molestar la âtencion

y fineza de Vuestra Reverencia âquien suplico

el disimulo, y muchos ôrdenes de

suâgrado, como â Dios que guarde

la Vida de Vuestra Reverencia los muchos años que

puede. San Luis y Mayo 22

de 1758./.

Besa Las Manos de Vuestra Reverencia

su Amigo afectuoso y muy servidor

\{rubrica\} Diego Ôrtiz

Parrilla 


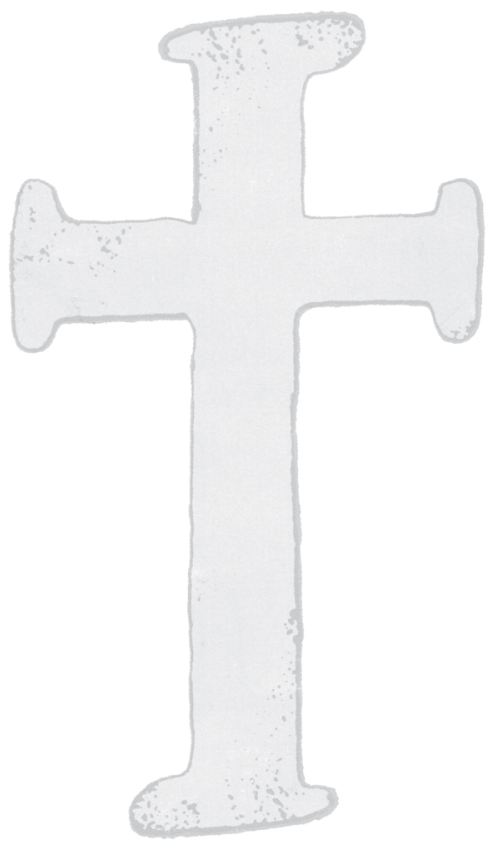




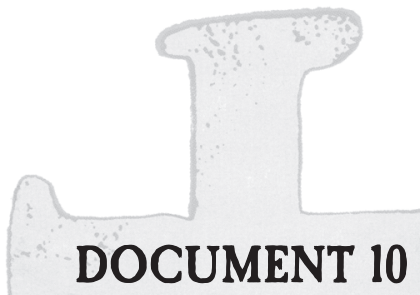

Letter from Colonel Parrilla to the Viceroy Marqués de las Amarillas July 4, 1758; ca. 4 months after the attack

Celaya Collection, Old Spanish Missions Collection, Our Lady of the Lake University, San Antonio 


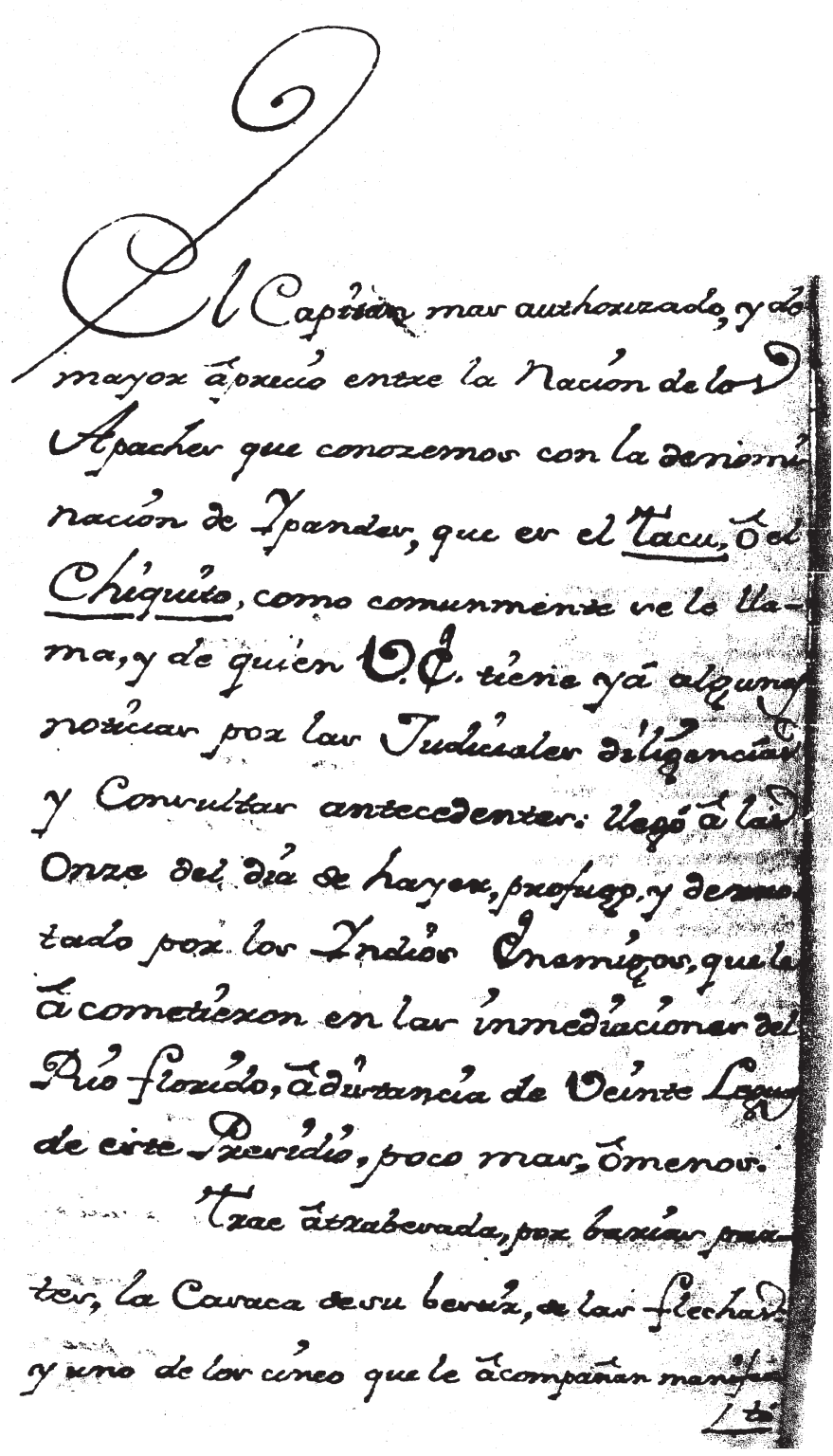


[fol. l $\mathrm{r}]$

\{Heading\} Excelentisimo Señor

II El Capitan mas authorizado, y do

mayor âprecio entre la Nacion de los

Apaches que conozemos con la denomi-

nacion de Ypandes, que es el Tacu, ô el

Chiquito, como comunmente se lo lla-

ma, y de quien Vuestra Excelencia tiene yá algunas

noticias por las Judiciales diligencías

y Consultas antecedentes: llegó â las

onze del dia de hayer, profugo y desmon-

tado por los Yndios Enemigos, que le

â cometieron en las inmediaciones del

Rio florido, â distancia de Veinte Leguas

de este Presidio, poco mas, ômenos.

II Trae âtrabesada, por barias par-

tes, la Casaca de su bestir, de las flechas:

y uno de los cinco que le âcompañan manifes- 
Spanish Colonial Documents Pertaining to Mission San Sabá

la Hexída de una Mala gice le ấbably wóp paxue del rovoxo.

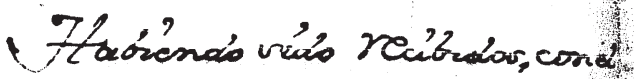

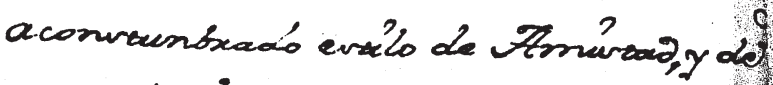

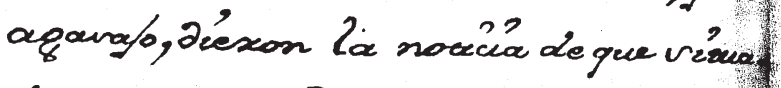
Lor en una torne dela esoprevada ted

ra, paxa haxex ve Caxexia de Zúbi lal.

tuexon Zexcador txen draw ha, ?

in 1.

ouna,y Cacuteionamente pox cxeccido n

10

mexo de Yondiós la Nacion Yylman

gre tambión áveinzio à la tracicion y"

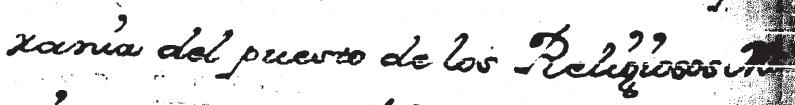

pionexas de ave - Xio.

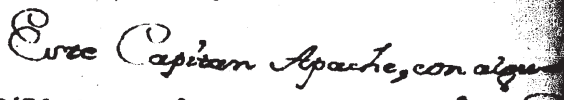

15

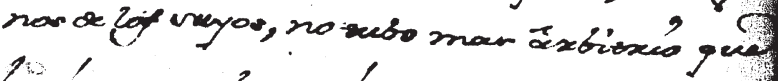

la tuqa que logro- hasex endme el devoxbo

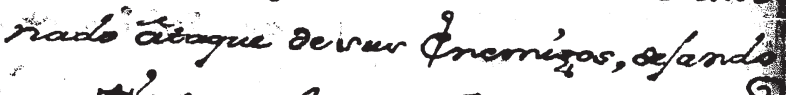

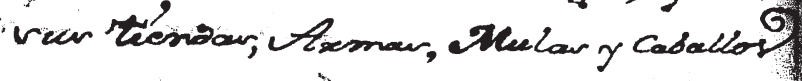

en pradiax de lair gue lor burcaban con el mayox rope. if.

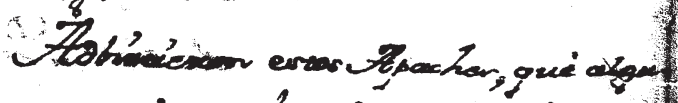

20

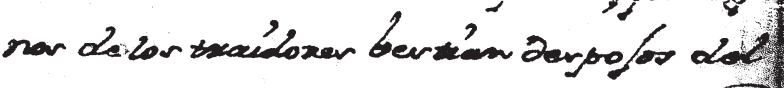

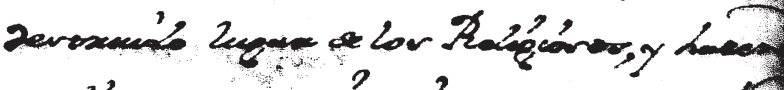

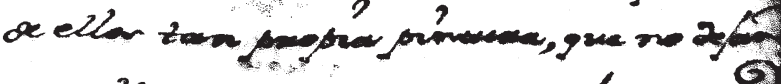

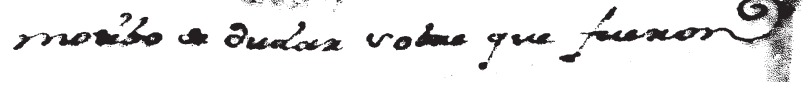




\section{[fol. lv]}

la Herida de una Bala que le âtrabesó parte del rostro.

II Habiendo sido recibidos con el aconstunbrado estilo de Amistad, y de agasajo, dieron la noticia de que situados en una Loma de la expresada tierra, para hazer su cazeria de Zibola, fueron zercados tres dias ha, improbisa, y cautelosamente por crecido numero de Yndios de la Nacion Yujuany que tambien âsistió â la traicion y tirania del puesto de los Religiosos Misioneros de este Rio.

II Este Capitan Apache con algunos de los suyos, no tubo mas ârbitrio que la fuga que logró hazer entre el desordenado âtaque de sus Enemigos, dejando sus tiendas, Armas, Mulas, y Caballos en poder de los que los buscaban con el mayor Vigor.

II Adbirtieron estos Apaches, qué algunos de los traidores bestian despojos del destruido lugar de los Religiosos, y hazen de ellos tan propria pintura, que no dejan motibo de dudas sobre que fueron 
complizer en la unow,y ôtsea âleboura." Orevitan iambion, alguna per

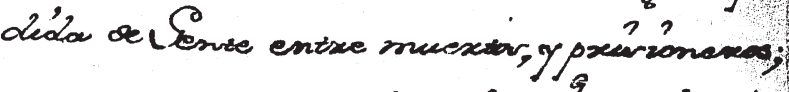

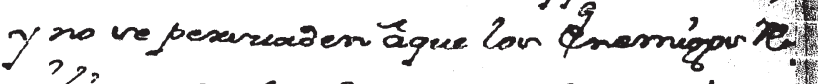
cebrexon daño alquno, no obutanis la tal. cual Defenera que predkexon inzexponex? Ferte Capizans juvereinas Com

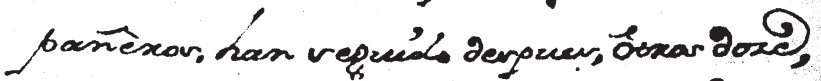

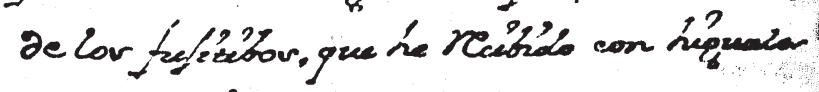
domaropacionen.

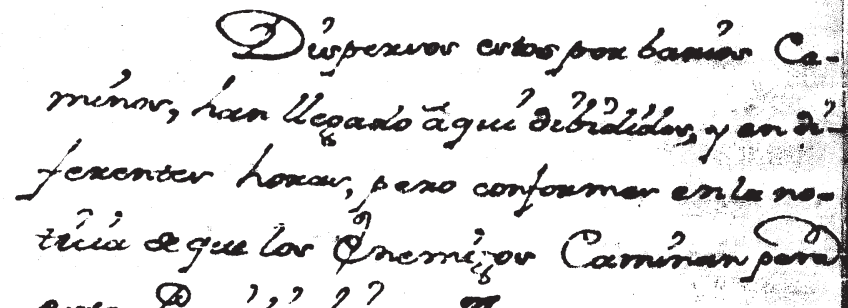

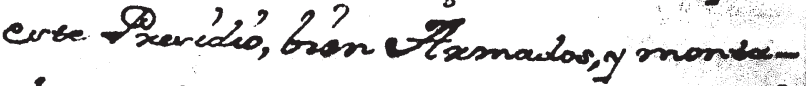

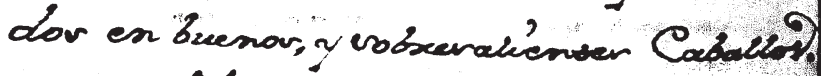

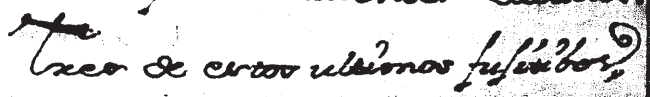
Oburexbaxion dar nochir ha, el mabímíoxios

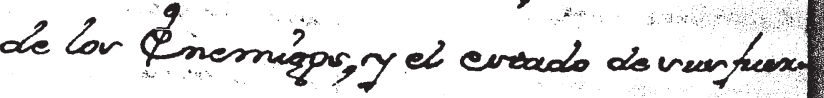

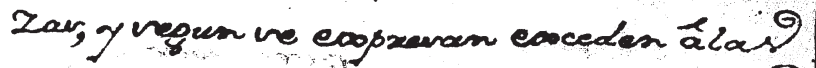

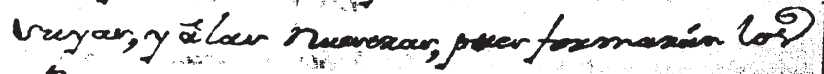
Basbaciar. el numeseo demour a dor mél.

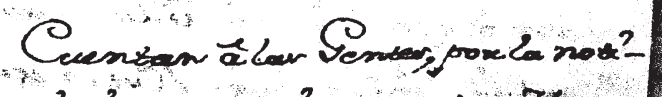

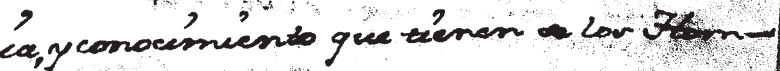

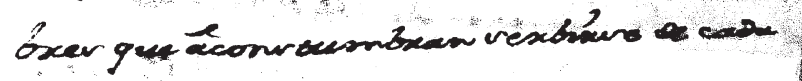




\section{[fol. 2r]}

complizes en la una y ôtra âlebosia.

II Cuentan tambien, alguna per-

dida de Gente entre muertos y prisioneros;

y no se persuaden âque los Enemigos re-

cibieron daño alguno, no obstante la tal

cual defensa que pudieron interponer.

II A este Capitan y sus cinco com-

pañeros, han seguido despues, ôtros doze, de los fujitibos, que he recibido con higuales demonstraciones.

II Dispersos estos por barios ca-

minos, han llegado âqui dibidídos, y en di-

ferentes horas, pero conformes en la no-

ticía de que los Enemigos Caminan para

este Presidio, bien Armados, y monta-

dos en buenos, y sobresalientes Caballos.

II Tres de estos ultimos fujitibos,

ôbserbaron dos noches ha, el mobimiento

de los Enemigos, y el estado de sus fuer-

zas, y segun se expresan exceden âlas

suyas, y â las nuestras, pues formarán los

Barbaros, el numero de mas de dos mil.

II Cuentan â las Gentes, por la noti-

cia, y conocimiento que tienen de los Hom-

bres que âconstumbran serbirse de cada 
Spanish Colonial Documents Pertaining to Mission San Sabá

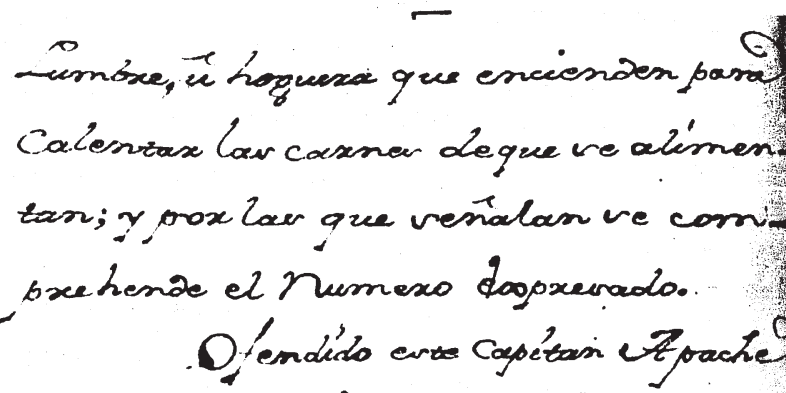

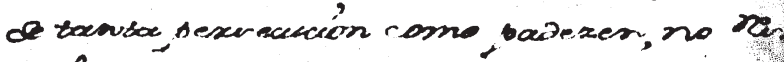
"bexa hoy ótxa courx, mner yue berpanza, $y$, dinsovercioner paxa ello, y en evta ózancin.

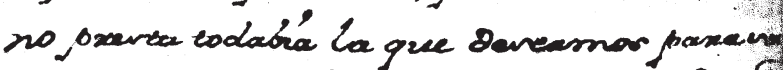
Rouscion, y âbecindamients a Míveones.

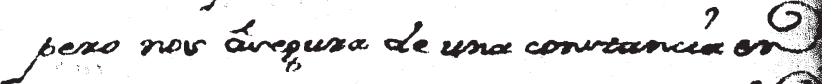

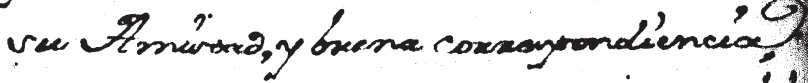

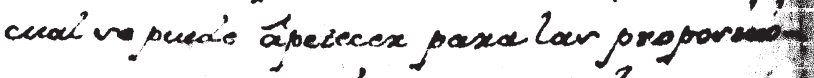

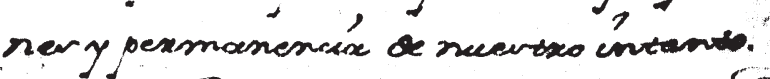

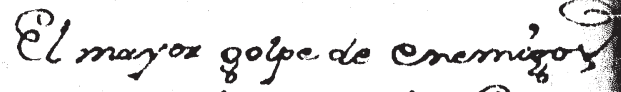

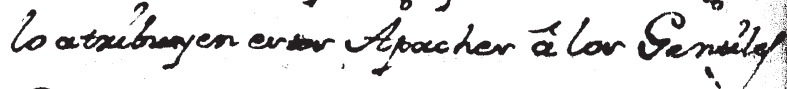
Cumvisichong puear de ellan volos contaxon

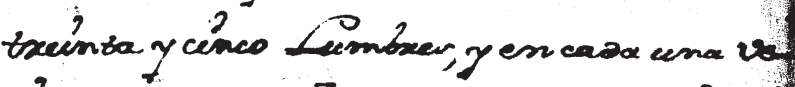

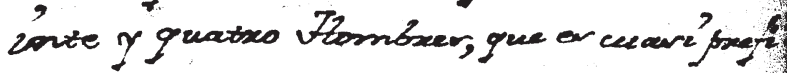

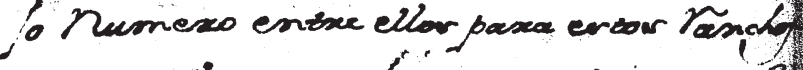
önepaxacioner que foxman en lar txamen? tor de vier maxe har guands low 2 ixejen 9

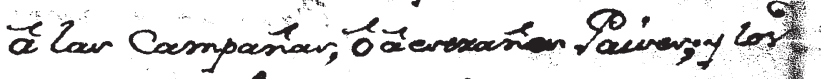
Nivtanzer énemigar, lor guesutan olan amaer Nocioner äliadan.

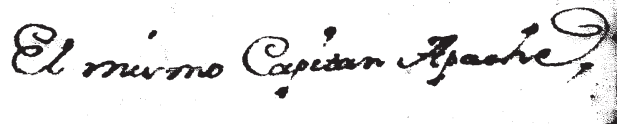




\section{[fol. 2v]}

\{right margin 2\}

Lumbre, ô hoguera que encienden para

calentar las carnes de que se alimen-

tan; y por las que señalan se com-

prehende el numero expresado.

II Ofendido este Capitan Apache

de tanta persecucion como padezen, no res-

pira hoy ôtra cosa, mas que benganza, y

disposiciones para ella, y en esta âtencion

no presta todabia la que deseamos para su

Reducion, y âbecindamiento â Misiones,

pero nos âsegura de una constancia en

su Amistad, y buena correspondiencia

cual se puede âpetecer para las proposicio-

nes y permanencía de nuestro intento.

II El mayor golpe de enemigos,

lo atribuyen estos Apaches â los Gentiles

Cumanches, pues de ellos solos contaron

treinta y cinco Lumbres, y en cada una ve-

inte y quarto Hombres, que es cuasi prefi-

jo Numero entre ellos para estos ranchos

ô separaciones que forman en los transi-

tos de sus marchas, quando las dirijen

â las campañas, ô â estrañ(^o)s Paises; y los

restantes Enemigos, los quentan de las

demas naciones âliadas.

II El mismo Capitan Apache, 


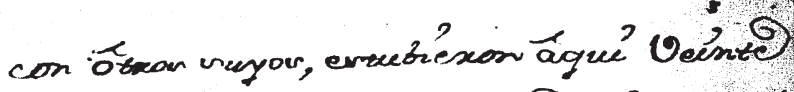

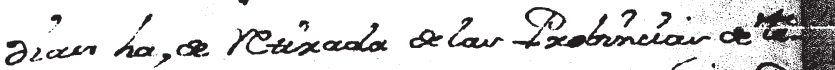
lan, y coahucilo en don de, prox lov muchos? - Vndior deve deboción gree congregép paxa lof Taxolees, y burctow, gue acconentumbxam ha-

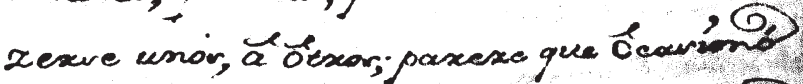

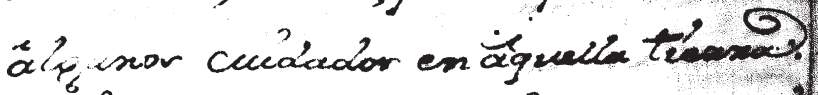

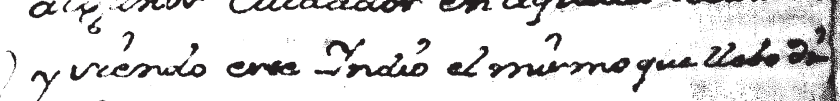
¿ho, y de quéen téene noxicí l.E. compone-

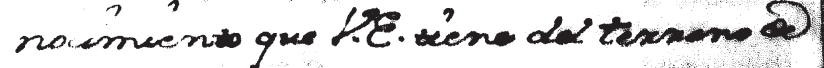

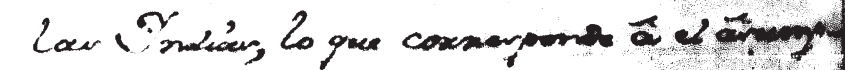
to. P

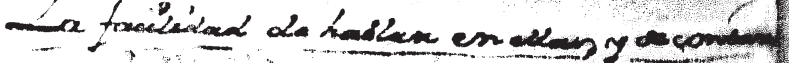

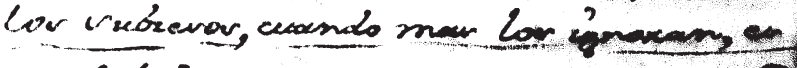

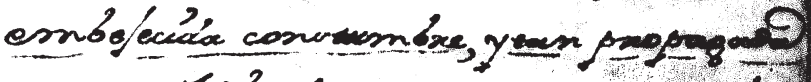

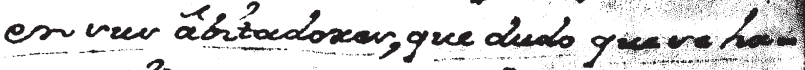

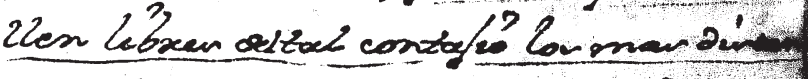

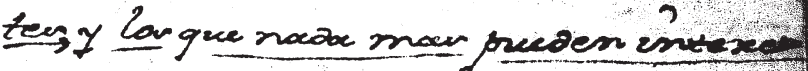
vex, que cosexenoondese à la inclinucion de pexaxbadorees y en ingucierov. Co obuzante la dértancé, engü

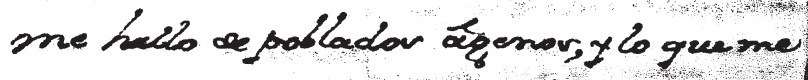

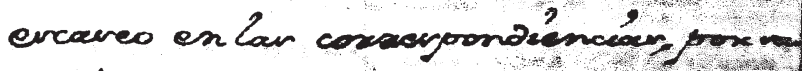


[fol. 3r]

\{left margin\} Lines 8 to 12 noted

con ôtros suyos, estubieron âqui Veinte dias ha, de retirada de las Probincias de tejas, y de coahuila en donde, por los muchos Yndios desu debocion que congregó para los tlatoles, y bisitas, que âconstumbran ha-

zerse unos, â ôtros; pareze que ôcasionó

âlgunos cuidados en âquella tierra:

y siendo este Yndio el mismo que llebo dicho, y de quien tiene noticia Vuestra Excelencia compre-

henderá su discrepcion, con el singular co-

nocimiento que Vuestra Excelencia tiene del terreno de

los Yndios, lo que corresponde â el âsumpto.

II La facilidad de hablar en ellos y de conducer

$\underline{\text { los subcesos, cuando mas los ignoran, es }}$

embejecida cons $(\wedge t)$ umbre, ytan propagada

en sus âbitadores, que dudo que se ha-

$\underline{\text { llen libres del tal contajio los mas distan- }}$

tes, y los que nada mas pueden intere-

$\underline{\text { sar, que corresponder â la inclinacion }}$

de perturbadores y de inquietos

II No obstante la distancia en que

me hallo de pobladores âgenos, y lo que me escaseo en las correspondencias, por satisfacer mi Genio desprendido de quimericos encuentros, y de recibir ôtras noti cias 


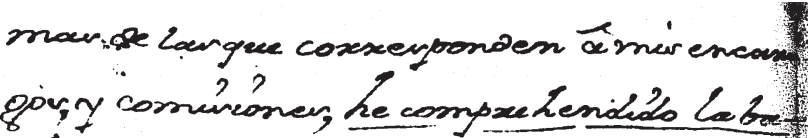

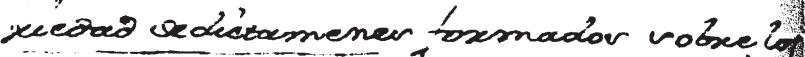

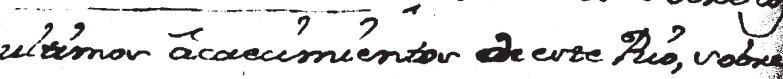

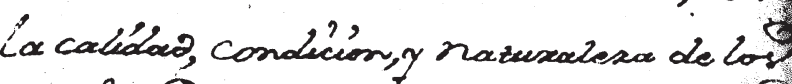

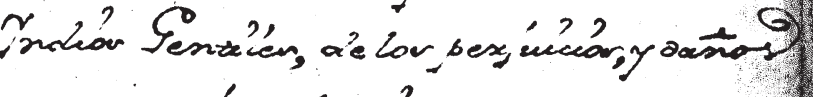

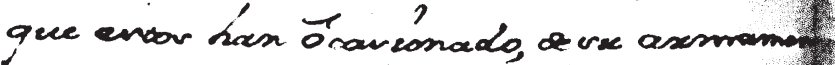

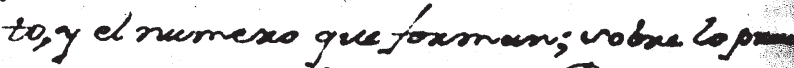

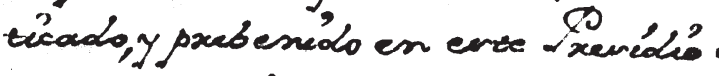

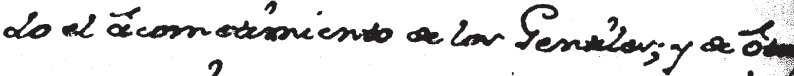

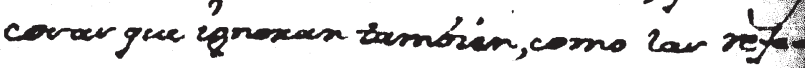

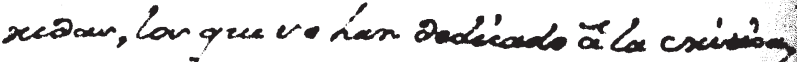

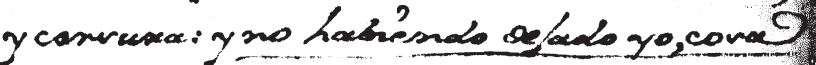

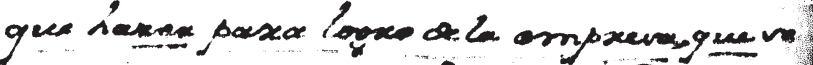

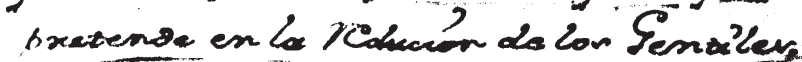
y convexbación del preverionad. Texsenge

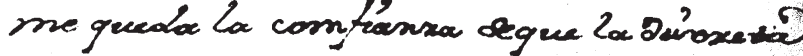

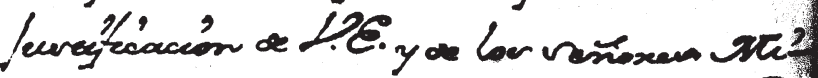

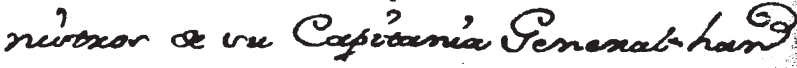

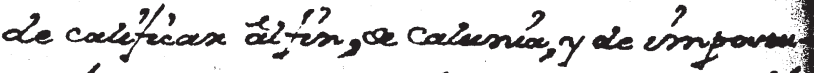

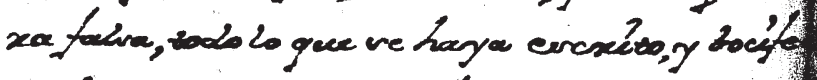

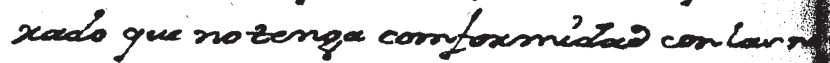

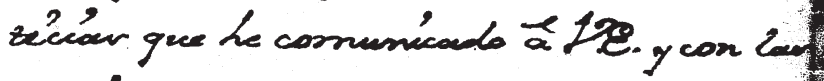
greviexe quente enadelasute a gualgun?

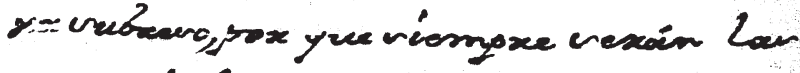

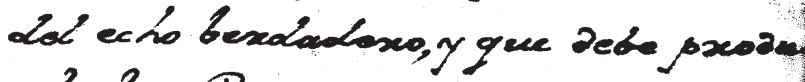

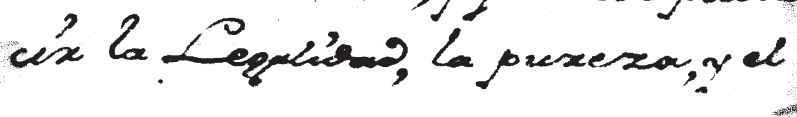


[fol. 3v]

\{top left margin\} 4

mas de las que corresponden â mis encargos, y comisiones, he comprehendido la bariedad de dictamenes formados sobre los ultimos âcaecimientos de este Rio, sobre la calidad, condicion, y naturaleza de los Yndios Gentiles, de los perjuicios, y daños que estos han ôcasionado, de su armamento, y el numero que forman; sobre lo practicado, y prebenido en este Presidio cuando el âcometimiento de los Gentiles; y de ôtras cosas que ignoran tambien, como las referidas, los que se han dedicado â la critica, y censura: y no habiendo dejado yo, cosa que hazer para logro de la empresa, que se pretende en la reducion de los Gentiles, y conserbacion del prosesionado Terreno; me queda la confianza de que la discreta justificacion de Vuestra Excelencia y de los señores Ministros de su Capitania General han de calificar âlfin, de calunia, y de impostura falsa, todo lo que se haya escrito, y bociferado que no tenga comformidad con las noticias que he comunicado â Vuestra Excelencia y con las que diere quenta en adelante de qualquiera subceso, por que siempre serán las del echo berdadero, y que debe producir la Legalidad, la pureza, y el 
Spanish Colonial Documents Pertaining to Mission San Sabá

rempecto. 4

Todo el manglo obvextido devds me

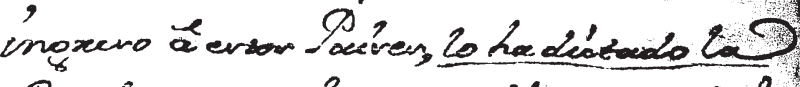

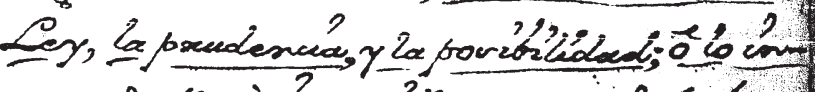

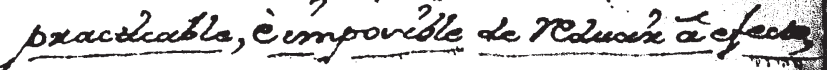
prox. Lo grap paxese gue no preeden temese lo.

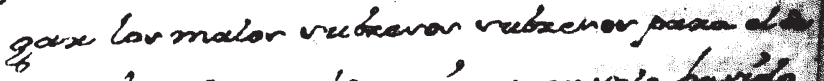

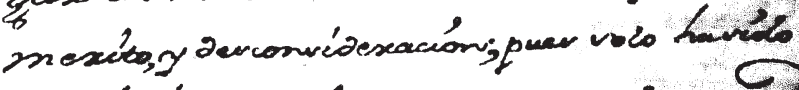

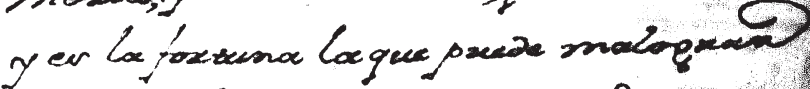

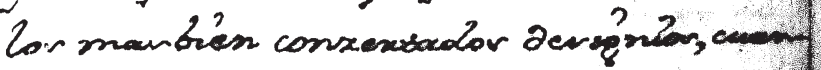

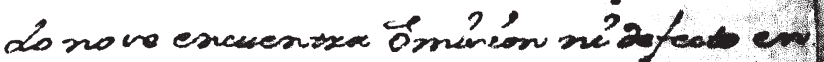

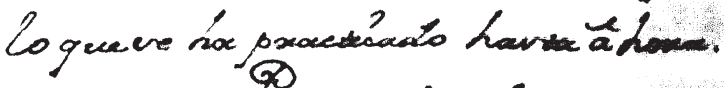

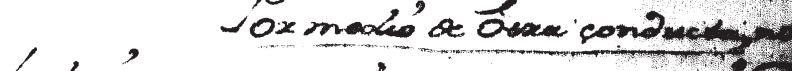

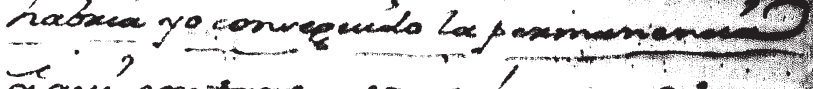

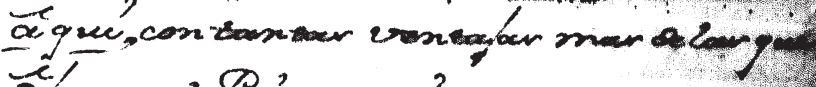

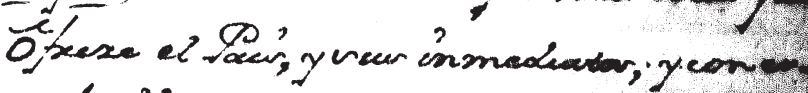

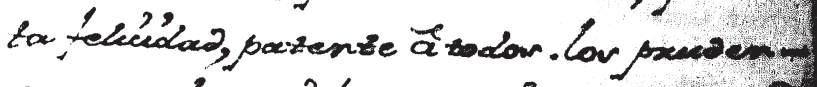

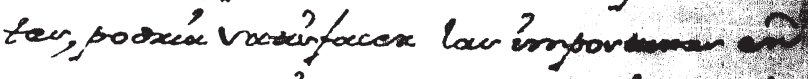
Cawo degue ve intexpongan pox lor me IV

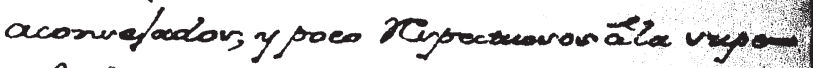
xioxidat die.

Lor pxoyectar,y convecoscínen en

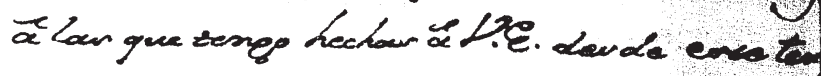
xeno grue en el ausempor, er elembadílo er al

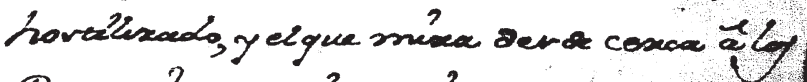

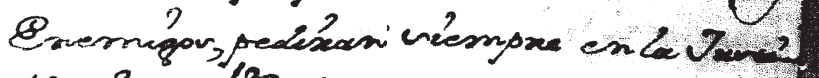

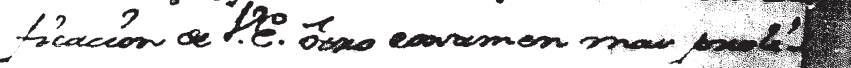

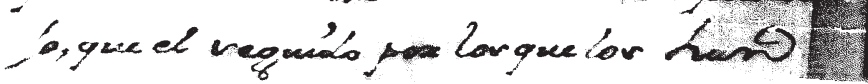




\section{[fol. 4r]}

respecto.

II Todo el manejo obserbado desde mi

ingreso â estos Paises, lo ha dictado la

Ley, la prudencia, y la posibilidad; ô lo im-

practicable, è imposible de reducir â efecto,

por lo que pareze que no pueden tener lu-

gar los malos subcesos $\left({ }^{\wedge}\right.$ subcesos) para el de-

merito, y desconsíderacion, pues solo ha sido

y es la fortuna la que puede malograr

los mas bien conzertados designios, cuan-

do no se encuentra ômision ni defecto en

lo que se ha practicado hasta â hora.

\section{Por medio de ôtra conducta, no}

habria yo conseguido la permanencia

â qui, con tantas ventajas mas de las que

offreze el Pais, y sus inmediatos; y con es-

ta felicidad, patente â todos los pruden-

tes podria satisfacer las imposturas en

caso de que se interpongan por los mal

aconsejados, y poco respectuosos âla supe-

rioridad de Vuestra Excelencia.

\section{Los proyectos, y contrarias instancias}

â las que tengo hechas â Vuestra Excelencia desde este ter-

reno que es el asumpto es el imbadido, es el

hostilizado, y el que mira desde cerca â los

Enemigos, pediran siempre en la Justi-

ficacion de Vuestra Excelencia ôtro exsamen mas proli-

jo, que el seguido por los que los han 


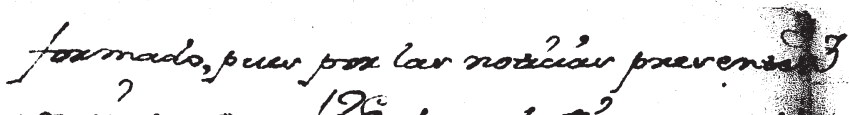

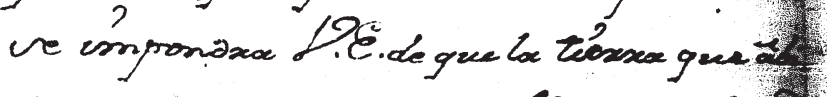

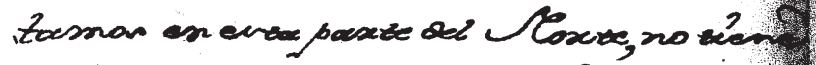

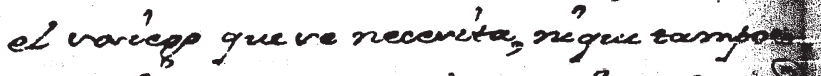
pexmetes urew altexacionen, ne conténum

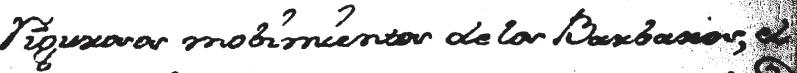

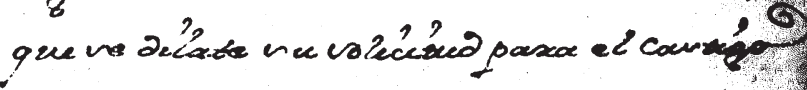

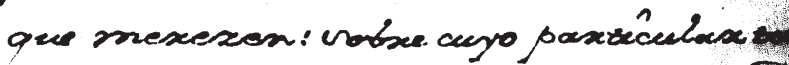

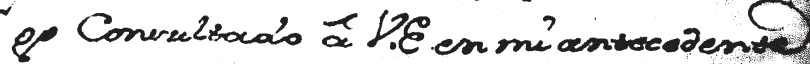
de öcho of abxel, lo gere me pascere cambe-

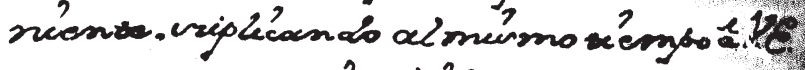

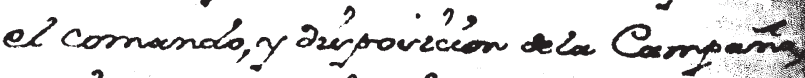

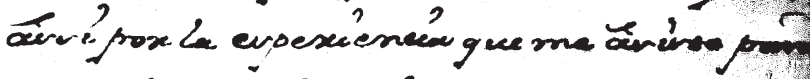

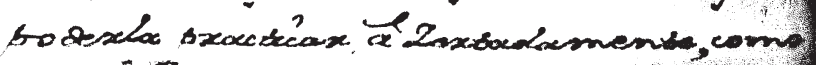

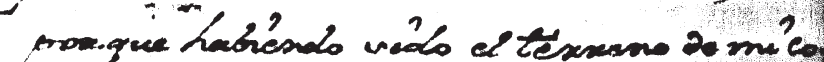

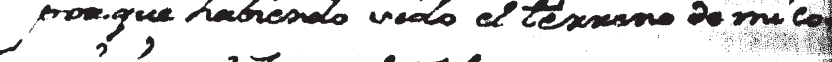

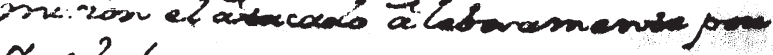
$r$ ?

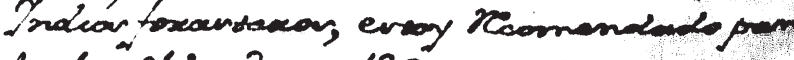
La juréfécacion Pl.e. CHechor ficescon lov Kndion âle?

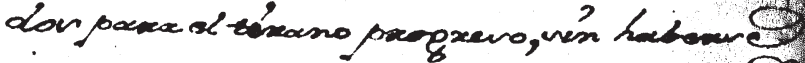

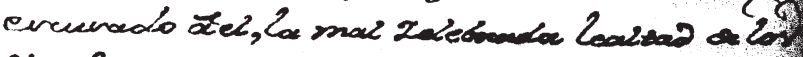

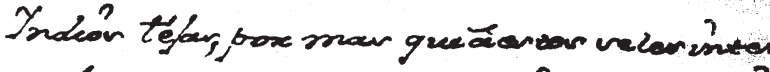

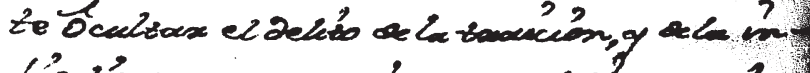

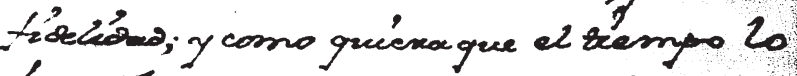

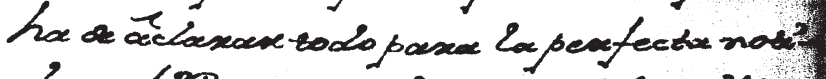

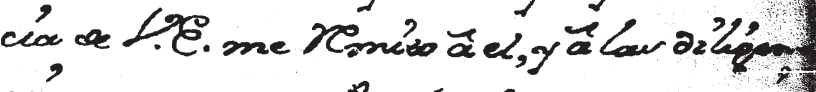

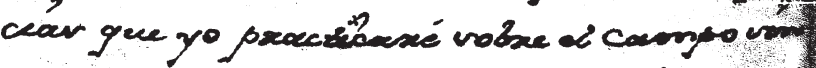

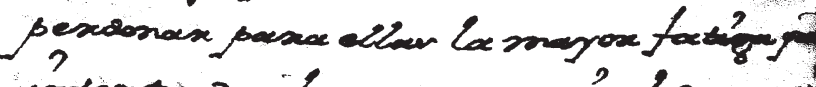

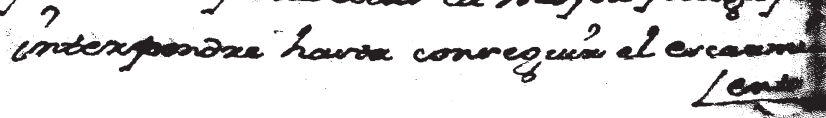




\section{[fol. 4v]}

\{right margin\} 3

formado, pues por las noticias presentes

se impondra Vuestra Excelencia de que la tierra que âbi-

tamos en esta parte del Norte, no tiene

el sosiego que se necesita, ni que tampoco

permiten sus âlteraciones, ni continuos

rigurosos mobimientos de los Barbaros, el

que se dilate su solicitud para el castigo

que merezen: sobre cuyo particular ten-

go consultado â Vuestra Excelencia en mi antecedente

de ôcho de abril, lo que me parece combe-

niente. suplicando al mismo tiempo â Vuestra Excelencia

el comando, y disposicion de la Campaña,

âssi por la esperiencia que me âsiste para

poderla practicar â zertadamente, como

por que habiendo sido el terreno de mi co-

mision el âtacado âlebosamente por

Yndios forasteros, estoy recomendado para

la justificacion de Vuestra Excelencia.

II Muchos fueron los Yndios âlia-

dos para el tirano progreso, sin haberse

escusado â el, la mal zelebrada lealtad de los

Yndios tejas, por mais que â estos seles inten-

te ôcultar el delito de la traicion, y de la in-

fidelidad; y como quiera que el tiempo lo

ha de âclarar todo para la perfecta noti-

cia de Vuestra Excelencia me remito â el, y â las diligen-

cias que yo practicaré sobre el campo sin

perdonar para ellas la mayor fatiga que

interpondre hasta conseguir el escarmi-

ento. 
Spanish Colonial Documents Pertaining to Mission San Sabá

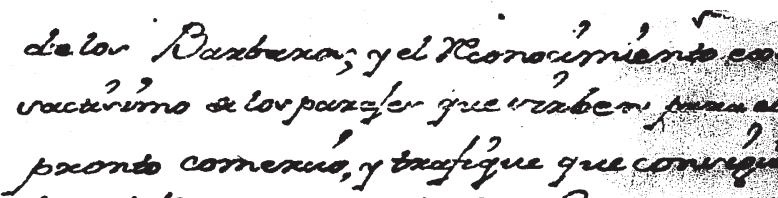

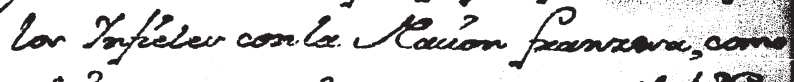

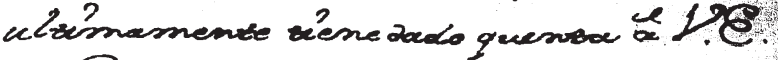

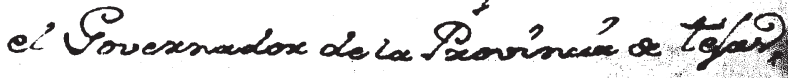

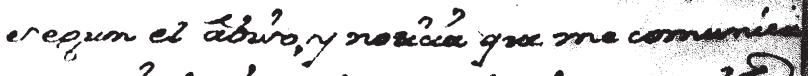

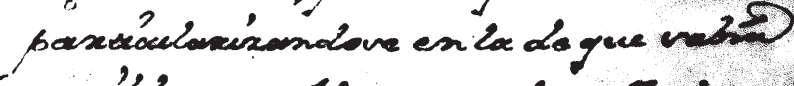

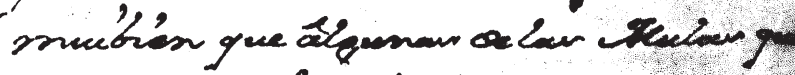

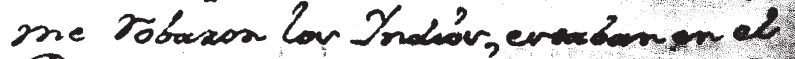

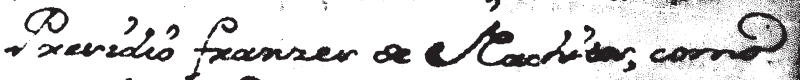

en orxin Doblaswon feansenes atg on

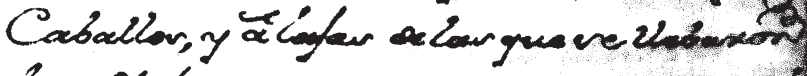

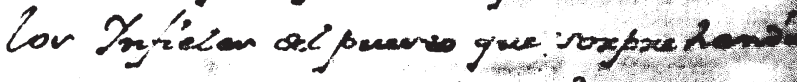
xo ...pox medis do un áledmen'

\section{Q} Saco ma caskyion minins

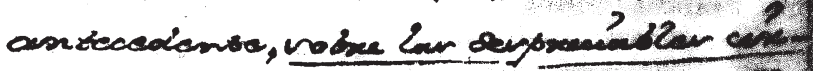

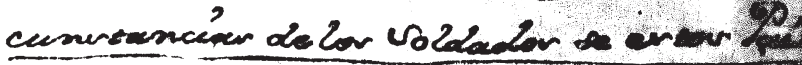

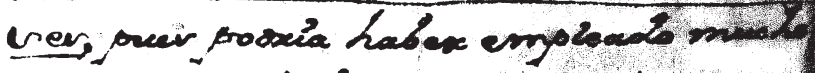

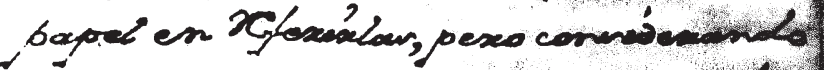

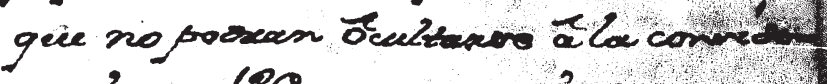

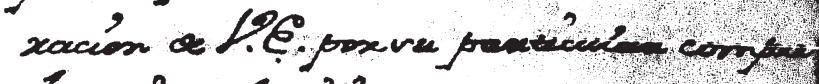
henerion, abne'pox enzonsen, cons 2

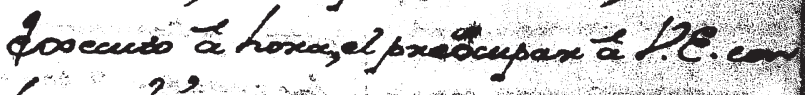
la noreas.

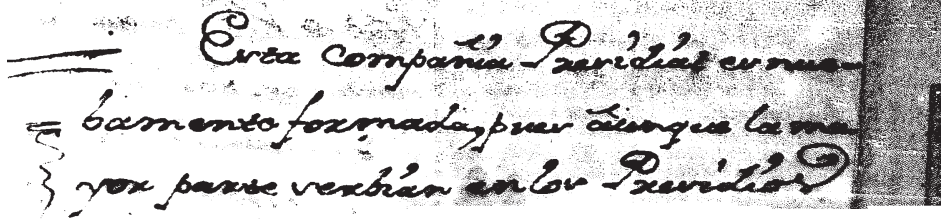




\section{[fol. 5r]}

\{left margin\} lines 26 through 28 noted

de los Barbaros; y el reconocimiento ex-

sactisimo de los pasajes que sirben para el

pronto comercio, y trafique que consiguido

los Ynfieles con la Nacion franzesa, como

ultimamente tiene dado quenta â Vuestra Excelencia

el Governador de la Provincia de tejas

segun el âbiso, y noticia que me comunicou

particularizandose en la de que sabia

muibien que âlgunas de la Mulas que

me robaron los Yndios, estaban en el

Presidio franzes de Nachitos, como

en ôtr(^as) Poblazones franzesas, âlgunos

Caballos, y âlajas de las que se llebaron

los Ynfieles del puesto que sorprehendie-

ron por medio de su âlebosia.

II Poco me explayé en mi citada

antecedente, sobre las despreciables cir-

cunstancias de los soldados de estos Pai

ses, pues podria haber empleado mucho

papel en Referirlas, pero considerando

que no podran ôcultarse â la conside-

racion de Vuestra Excelencia por su particular compre-

hension, ôbmiti por entonzes, como lo

executo â hora, el preocupar â Vuestra Excelencia con

la noticia.

II = Esta compañia Presidial es nue-

bamente formada, pues âunque la ma-

yor parte serbian en los Presidios 
Spanish Colonial Documents Pertaining to Mission San Sabá

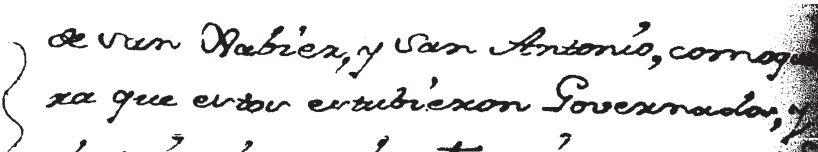

dictximador en lor texménar gue robits

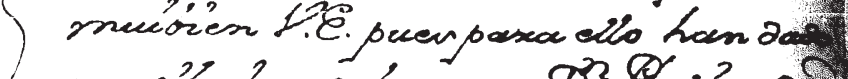

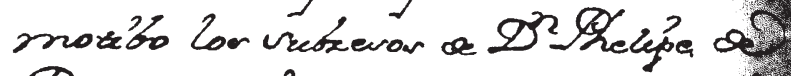

Pabagp. Capezam que fue a ven Dabt.

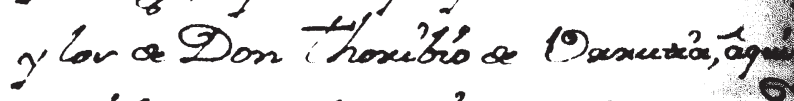
en del empleo de Cajízan del a van

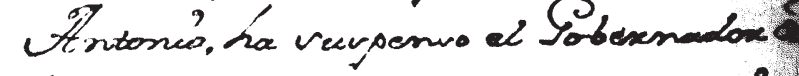

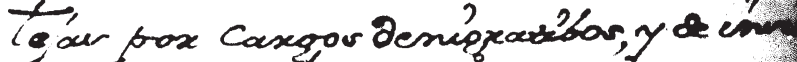

L'́ciencia, como tambien me ha come.

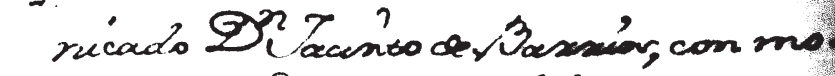

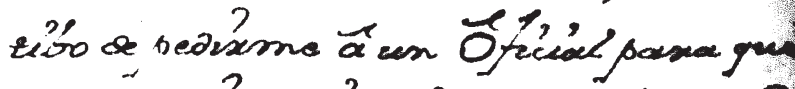

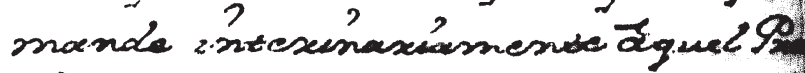

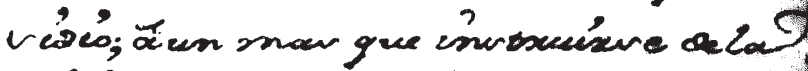

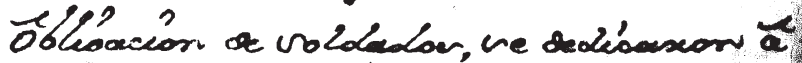

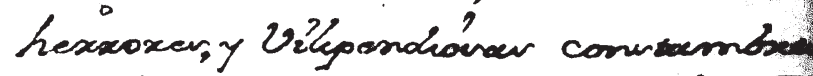

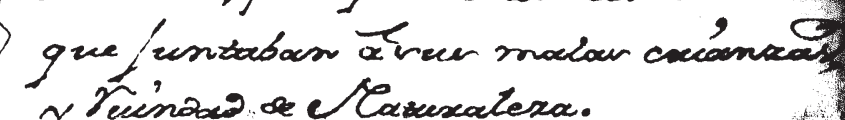
y Reinax de Cascatexa.

Uicon noticie de law nefoxidare

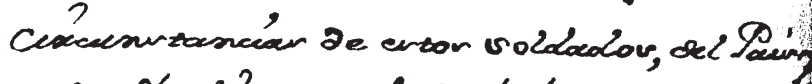

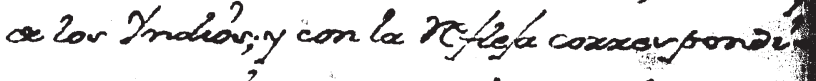

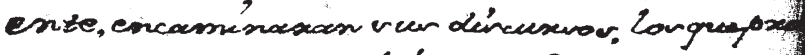

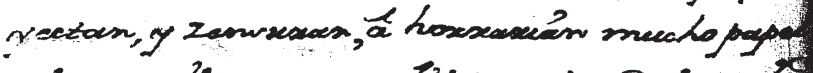

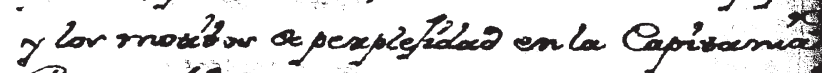

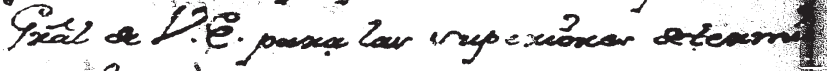

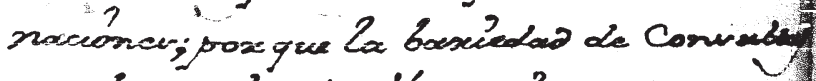

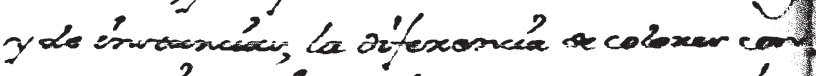

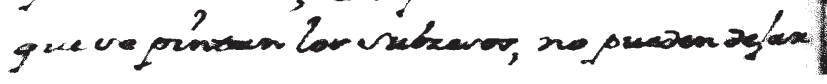




\section{[fol. 5v]}

\{left margin\} lines 1 through 19 noted

de San Xabier, y San Antonio como quiera que estos estubieron Governados, y doctrinados en los terminos que sabe muibien Vuestra Excelencia pues para ello han dado motibo los subcesos de Don Phelipe de

Rabago, Capitan que fue de San Xabier, y los de Don Thoribio de Urrutia, âquien en del empleo de Capitan del de San Antonio, ha suspenso el Gobernador de tejas por cargos denigratibos, y de insu-

ficiencia como tambien me ha comunicado Don Jacinto de Barrios, con motibo de pedirme â un Ôficial para que mande interinariamente âquel Presidio; â un mas que instruirse de la ôbligacion de soldados, se dedicaron â herrores, y vilipendiosas constumbres que juntaban â sus malas crianzas, y Ruindad de Naturaleza.

II Si con noticia de las referidas circunstancias de estos soldados, del Pais y de los Yndios; y con la refleja correspondiente, encaminascen sus discursos, los que proyectan, y [???zensuran], â horrarian mucho papel y los motibos de perplejidad en la Capitania

General de Vuestra Excelencia para las superiores determinaciones; por que la bariedad de consultas y de instancias, la diferencia de colores con que se pintan los subcesos, no pueden dejar 
Spanish Colonial Documents Pertaining to Mission San Sabá

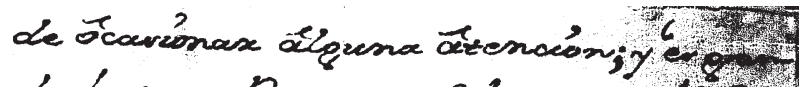

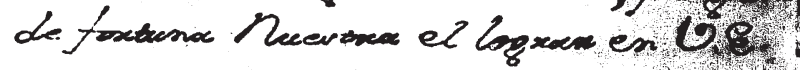

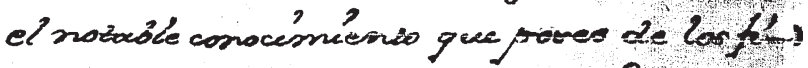

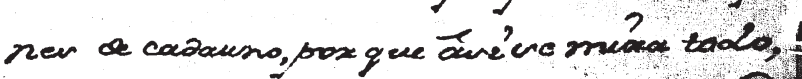

pxontamenis auspachado,y pxezenino?

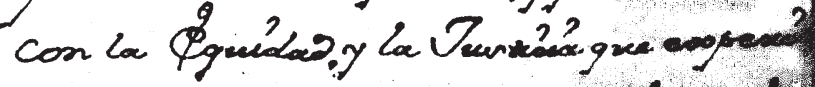

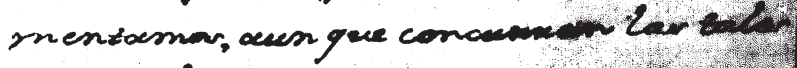

conessecsuciádedan:

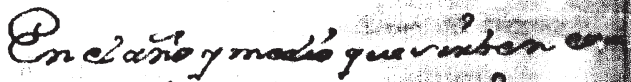

10

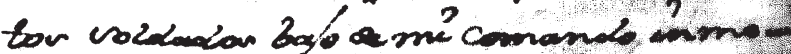

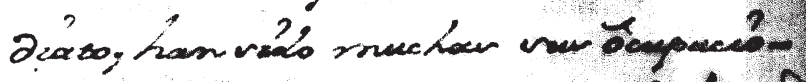

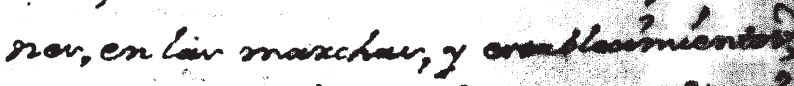

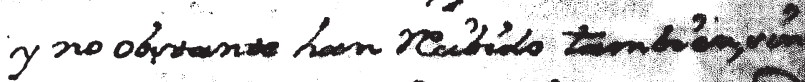

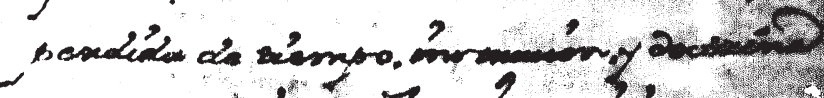

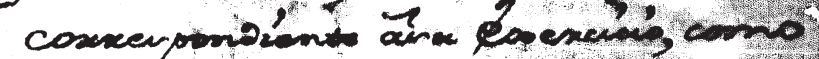

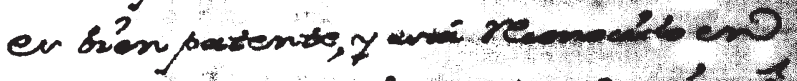

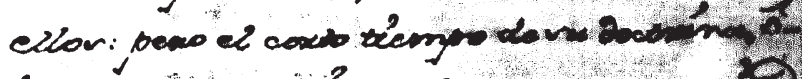

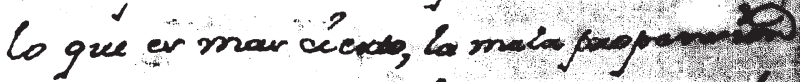

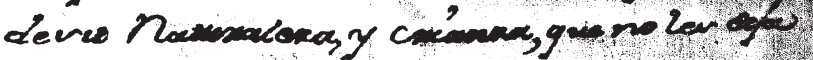

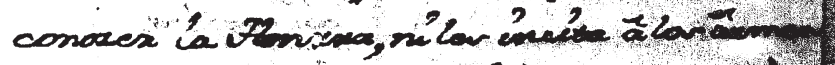

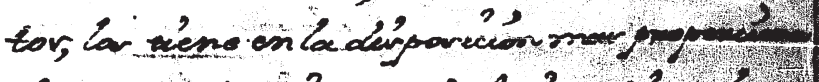

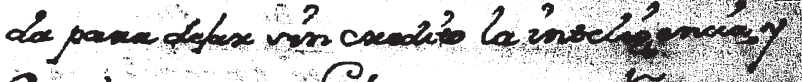

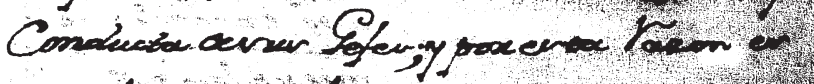

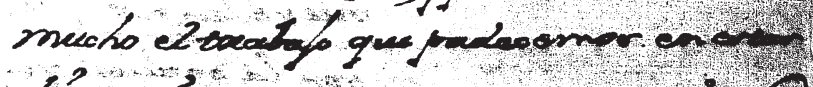
diónant, 1 , Srexia

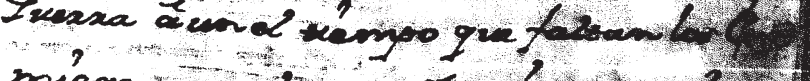

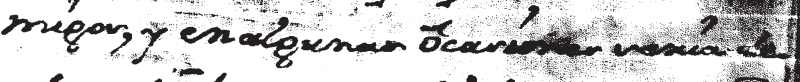

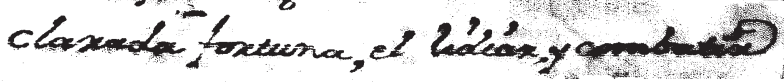




\section{[fol. 5v]}

\{top right margin\} 6

de ôcasionar âlguna âtencion; y es gran-

de fortuna nuestra el lograr en Vuestra Excelencia

el notable conocimiento que porvee de las fi-

nes de cadauno, por que âsi se mira todo,

prontamente despachado, y prebenido

con la Equidad, y la Justicia que experi-

mentamos, aun que concurran las tales

contrariedades:

II En el año y medio que sirben es-

tos soldados bajo de mi comando inme-

diato, han sido muchas sus ôcupacio-

nes, en las marchas, y establecimientos,

y no obstante han recebido tambien, sin

perdida de tiempo, instruccion, y doctrina

correspondiente âsu Exercicio, como

es bien patente, y está reconocido en

ellos: pero el corto tiempo de su doctrina â

lo que es mas cierto, la mala propensión

de su $\mathrm{Na}\left({ }^{\wedge} \mathrm{tu}\right)$ raleza, y crianza, que no les deja

conocer la [Honrra], ni los incita â los âumen-

tos, los tiene en la disposicion mas proporciona-

da para dejar sin credito la inteligencia y

Conducta de sus Gefes; y por esta Razon es

mucho el trabajo que padecemos en estas

distancias, y estamos en una continua

Guerra â un el tiempo que faltan los en-

migos y en algunas ôcasiones seria de-

clarada fortuna, el lidiar, y combater 
Spanish Colonial Documents Pertaining to Mission San Sabá

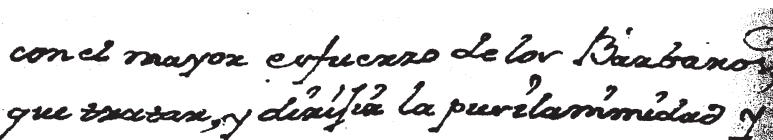
abandono de Nuenere Troper.

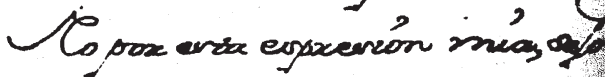
de conozex, que el - Dewridis de Uan Luér Le low Mtrmasullar pued vexbix' hoy of

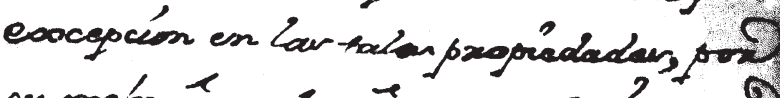

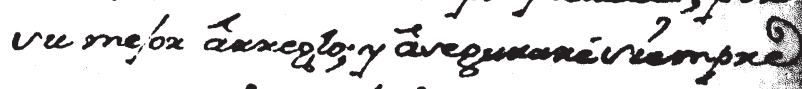

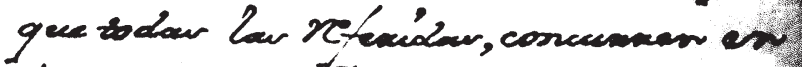

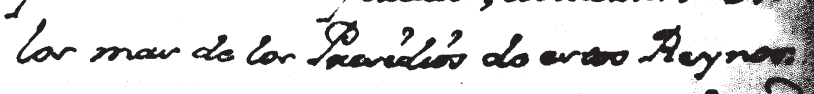

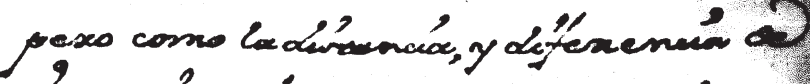
Qnemigor, haxen conarex y vank's man

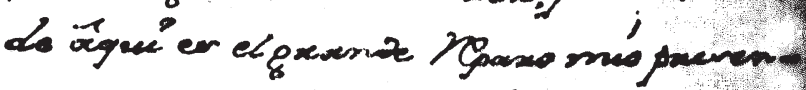

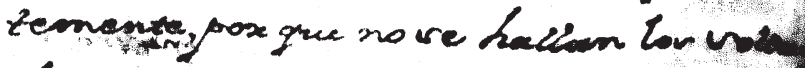
dor como von necenosxing y lor encomé gor ve encuentxan con lar venutal

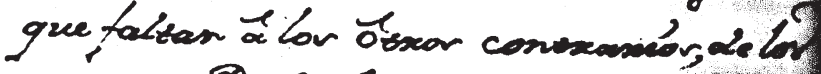

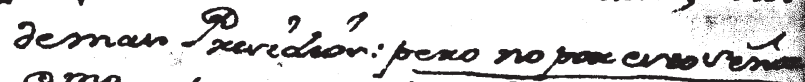
emo

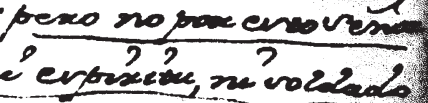

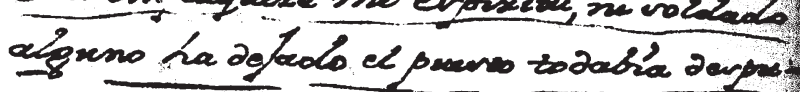
en de la txacídora entaceda Le lar Backab xor, exmedes de Igmella converenocions?

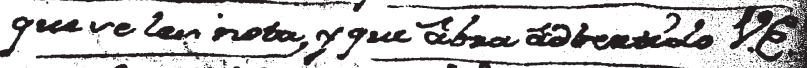

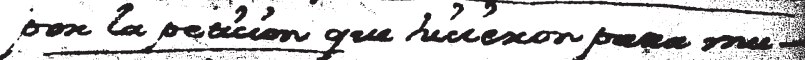

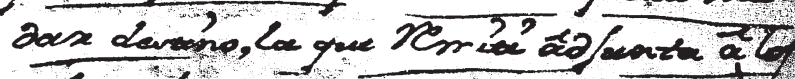

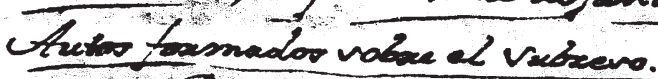

Po centa pexmanemo y lede-ben 6 1. 
[fol. 6r]

con el mayor esfuerzo de los Barbaros,

que tratar, y dirijir la pusilamimidad [pusilaminidad] y

abandono de nuestra tropa.

II No por esta expresion mia, dejo

de conozer qué el Presidio de San Luis

de las Amarillas puede serbir hoy de

excepcion en las tales propiedades por

su mejor ârreglo; y âseguraré siempre

que todas las referidas, concurren en

los mas de los Presidios de estos Reynos:

pero como la distancia, y diferencia de

Enemigos, hazen conozer, y sentir man-

de âqui es el grande Reparo mio presen-

temente, por que no se hallan los solda-

dos como son necesarios, y los enemi-

gos se encuentran con las ventajas

que faltan â los ôtros contrarios, de los

demas Presidios: pero no por esto Señor

Excelentisimo emflaquire mi espiritu, ni soldado

alguno ha dejado el puesto todabia despu-

es de la traidora entrada de los Barba-

ros, enmedio de âquella consternacion

que se les nota, y que âbra âdbertido Vuestra Excelencia

por la peticion que hicieron para mu-

dar destino, la que remiti âdjunta â los

Autos formados sobre el subceso.

II Para esta permanencia suya,

y la de todos en el terreno que ôcupamos 


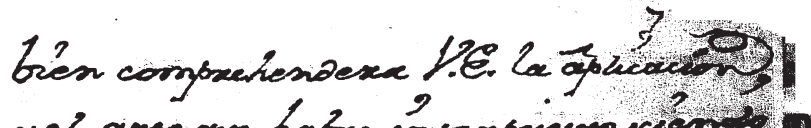

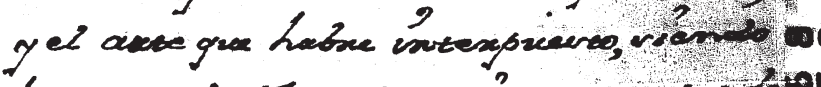

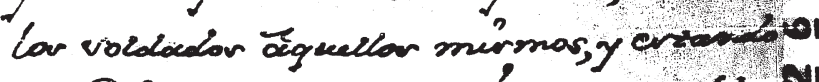

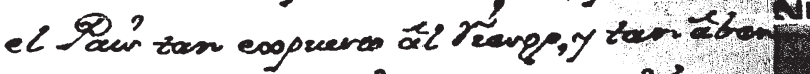

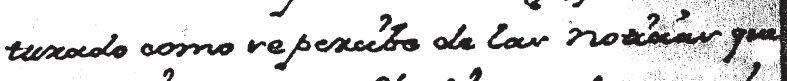
comuncean ever Inoliós, y de lo gue vela.

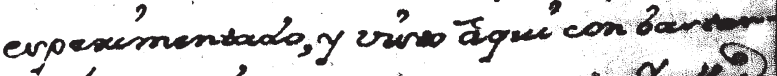

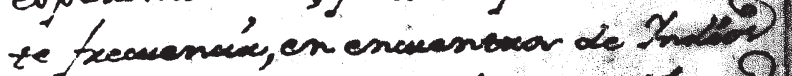

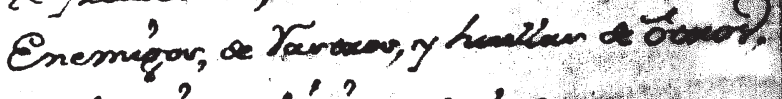

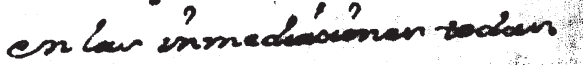

$$
\text { Cevar nowisine congue } 2 y
$$

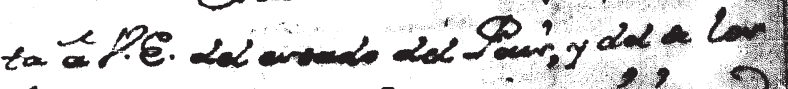

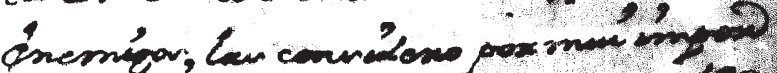

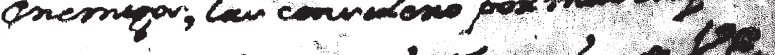

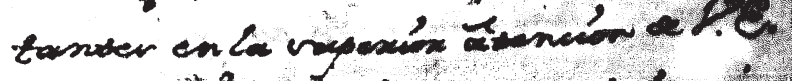

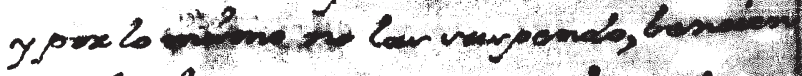

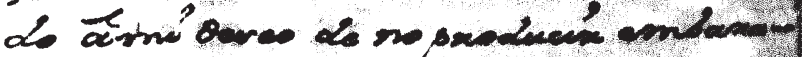

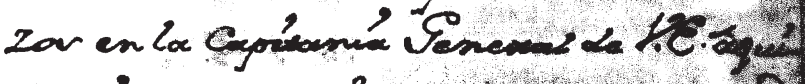

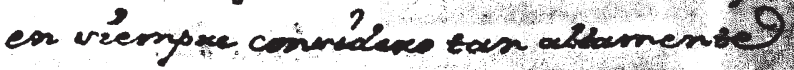
expreadis $8, \ldots, 2,1$

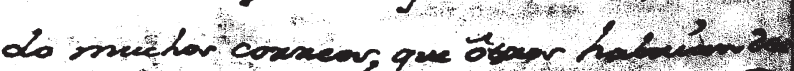

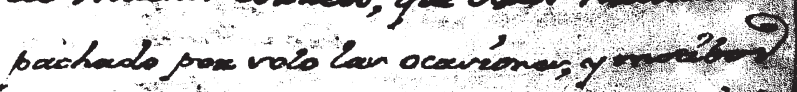

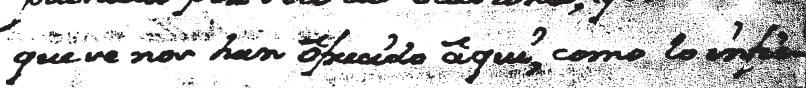

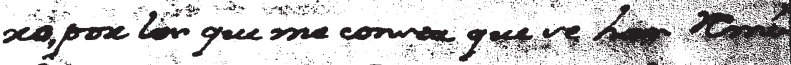

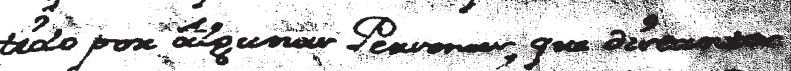

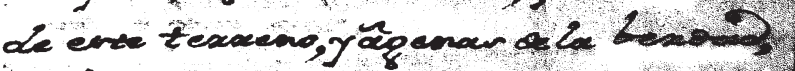

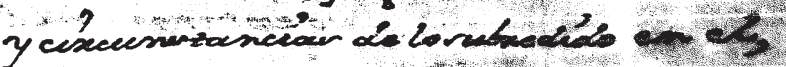

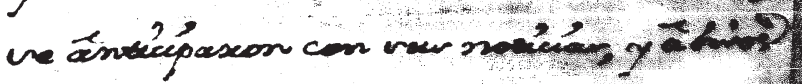




\section{[fol. 6v]}

bien comprehendera Vuestra Excelencia la âplicacion,

y el arte que habre interpuesto siendo

los soldados âquellos mismos, y estando

el Pais tan expuesto âl riesgo, y tan âben-

turado como se percibe de las noticias que

comunican estos Yndios, y de lo que se ha

esperimentado, y visto âqui con bastan-

te frecuencia, en encuentros de Yndios

Enemigos, de Rastros, y huellas de ôtros,

en las inmediaciones todas.

II Estas noticias con que doy cuen-

ta â Vuestra Excelencia del estado del Pais, y del de los

enemigos, las considero por mui impor-

tantes en la superior âtencion de Vuestra Excelencia

y por lo (^mismo) no las suspendo, bencien-

do âmi deseo de no producir embara-

zos en la Capitania General de Vuestra Excelencia âqui-

en siempre considero tan altamente

empleada.

II Esta justa Reflexion mia â honrra-

do muchos correos, que ôtros habrian des-

pachado por solo las ocasiones, y motibos

que se nos han ôfrecido âqui, como lo infie-

ro, por lo que me consta que se han remi-

tido por âlgunas Personas, que distantes

de este terreno, y âgenas de la berdad,

y circunstancias de lo subcedido en el,

se ânticiparon con sus noticias, y âbisos 
Spanish Colonial Documents Pertaining to Mission San Sabá

gros tombien convédexo defexenu posdéenter zémpor.

$$
\text { Ynaryo una conder gue } x
$$

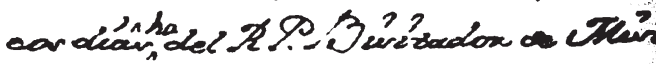

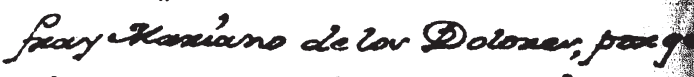

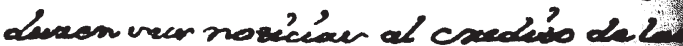

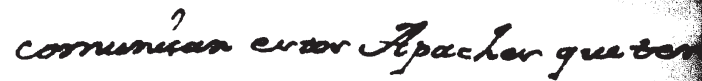

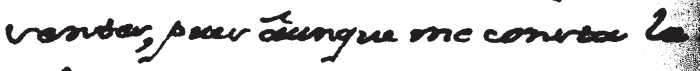
cha qre pacan ena cowe engree hen

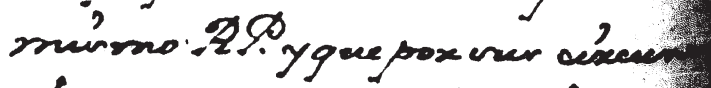

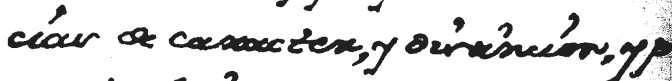

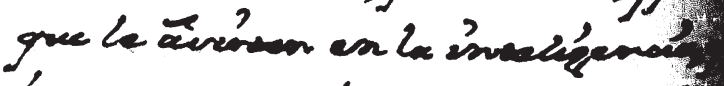
lo congues muator atorn a ecocuchen

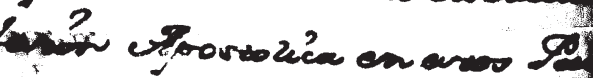

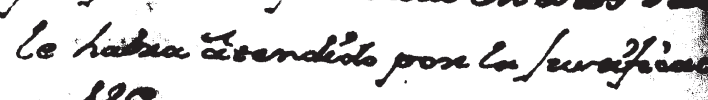

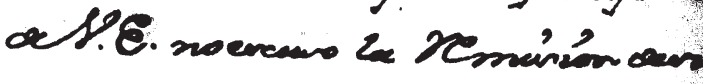

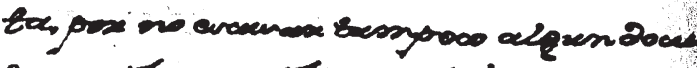

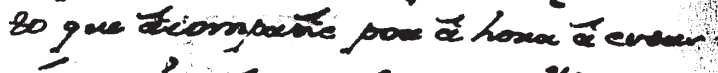

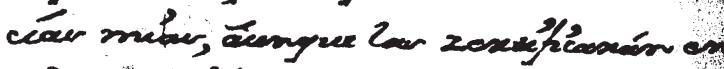

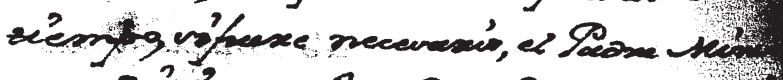

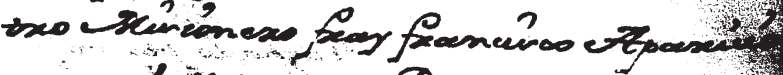

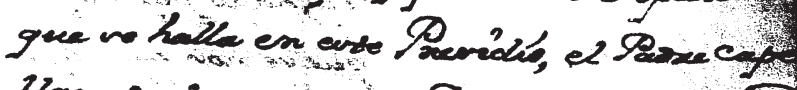

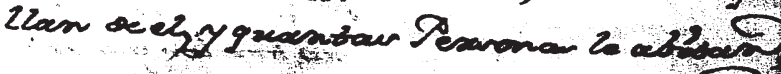

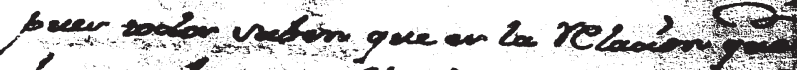

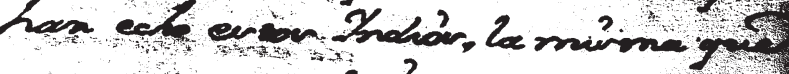

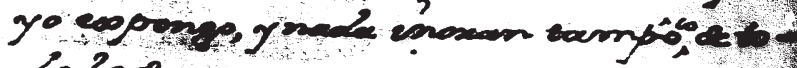
do 20 obenmen grese expeno.

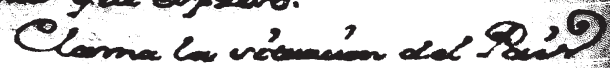




\section{[fol. 7r]}

que tambien considero diferentes âlos

mios, que se han comunicado en corres-

pondientes tiempos.

II Yncluyo una carta que recibi po-

cos dias (^ha) del Reverendo Padre Bisitador de Misiones

fray Mariano de los Dolores, por que con-

duzen sus noticias al credito de las que

comunican estos Apaches que tengo pre-

sentes, pues âunque me consta la mar-

cha que para esa corte emprehendio el

mismo Reverendo Padre y que por sus circunstan-

cias de caracter, y distincion, y por la

que le âsisten en la inteligencia, y ze-

lo con que muchos años â exercido su

profesion Apostolica en estos Pais [Paises], se

le habra âtendido por la justificacion

de Vuestra Excelencia no escuso la Remision de su car-

ta por no escusar tampoco algun documen-

to que âcompañe por â hora â estas noti-

cias mias, âunque las zertificarán en todo

tiempo, si fuere necesario, el Padre Minis-

tro Misionero fray francisco Aparicio

que se halla en este Presidio, el Padre Cape-

llan de el, y quantas Personas lo abitan,

pues todos saben que es la Relacion que

han echo estos Yndios, la misma que

yo expongo, y nada inoran tampo $\left({ }^{\wedge} \mathrm{co}\right)$ de to-

do lo demas que espreso.

II Clama la situacion del Pais 
Spanish Colonial Documents Pertaining to Mission San Sabá

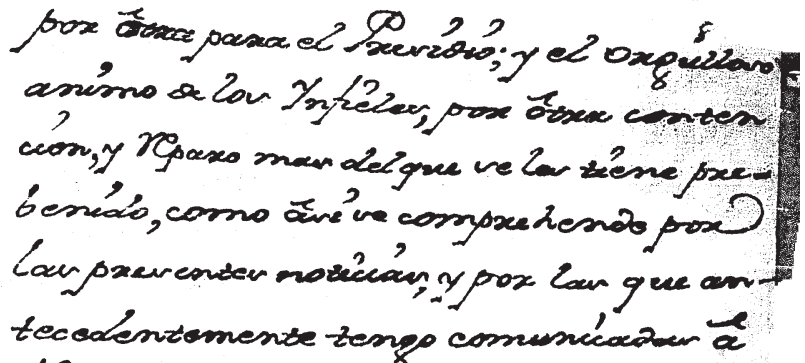

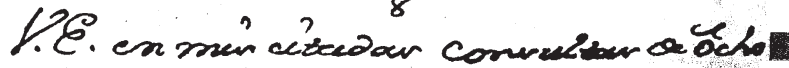

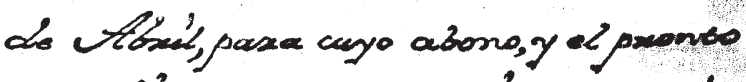
erpedieinos gue ve necorize, dayparto

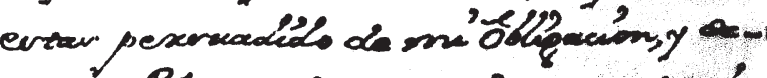
veo de gite todo lo cosumniz, y comben's

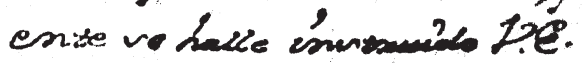

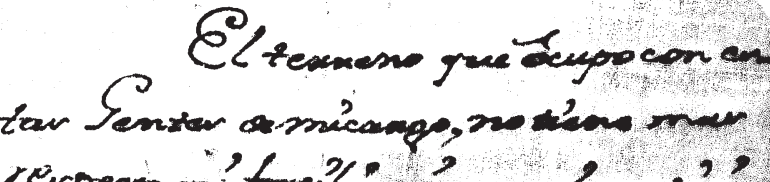

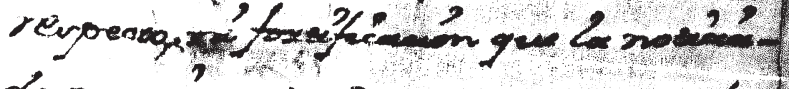

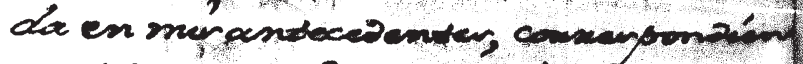

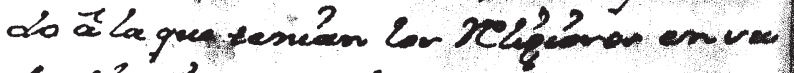

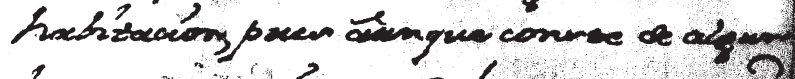

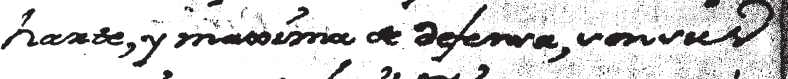
maxexciálan de frese" resina, pox gue niez

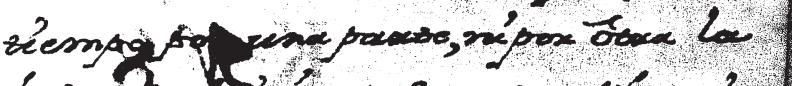

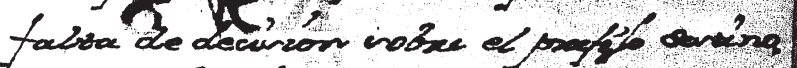

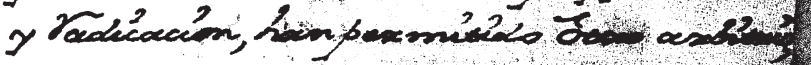
nitampoco el de ecopenden gever atell

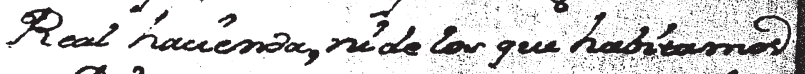

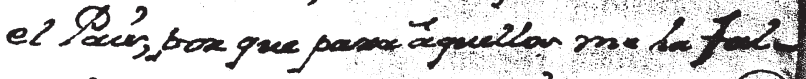

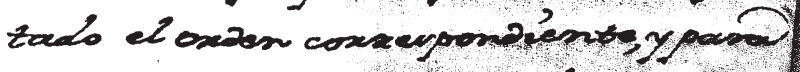

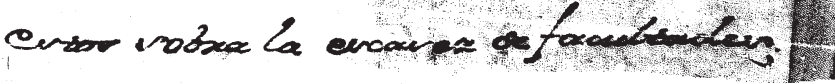




\section{[fol. 7v]}

por [??? ôtro] para el Presidio; y el orgulloso

animo de los Ynfieles, por ôtra conten-

cion, y reparo mas del que se les tiene pre-

benido, como âsi se comprehende por

las presentes noticias, y por las que an-

tecedentemente tengo comunicadas â

Vuestra Excelencia en mis citadas consultas de ôcho

de Abril, para cuyo abono, y el pronto

espediente que se necesita, despacho

estas persuadido de mi ôbligacion, y de-

seo de $\mathrm{q}^{\wedge}$ (ued)e todo lo exsencial, y combeni-

ente se halle instruido Vuestra Excelencia.

II El terreno que ôcupo con es-

tas Gentes de micargo, no tiene mas

respecto, ni fortificacion que la noticia-

da en mis antecedentes, correspondien-

do â la que tenian los Religiosos en su

habitacion, pues âunque con ser de algun

harte, y maxima de defensa, son sus

materiales de facil ruina, por que niel

tiempo po[r] una parte, ni por ôtra la

falta de decision sobre el prefijo destino,

y Radicacion, han permitido ôtro arbitrio

nitampoco el de expender gastos de la

Real hacienda, ni de los que habitamos

el Pais, por que para âquellos me ha fal-

tado el orden correspondiente, y para

estos sobra la escasez de facultades; 
Spanish Colonial Documents Pertaining to Mission San Sabá

5

prox logree ve podxo todexex much traxiempo, en cavo de atague, ccuannat

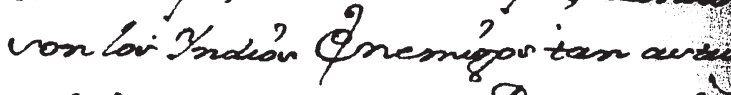
$y$ danis numexo of Dinonar the Zadan'y ex el Campo podecan caoposest th

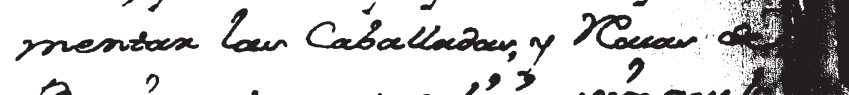
- Hawnonestor uxpexedicions weng gre 18

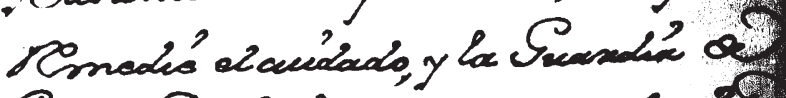
Sente Dewridiál que àsods ve ôpkell

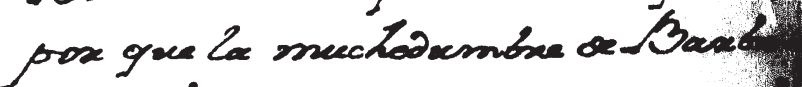

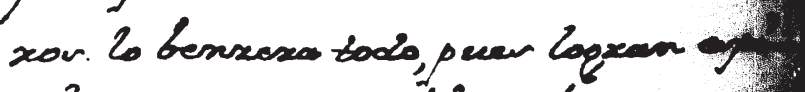

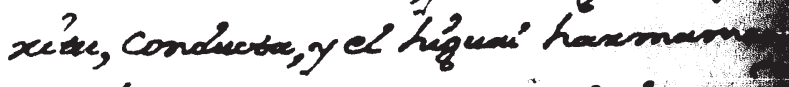

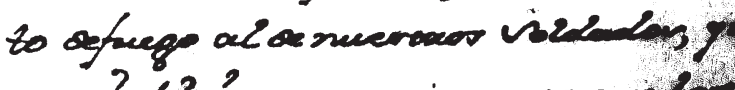

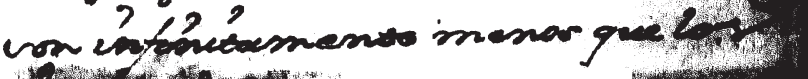
trt sod deow.

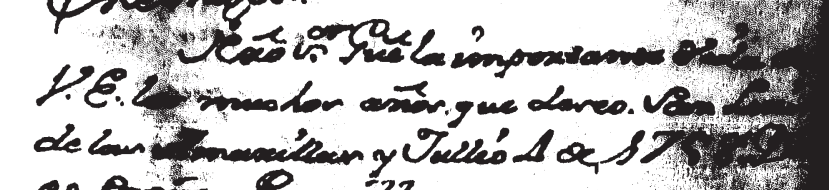
of Qser Leariz2 =

Kor 8 Lig 8

20

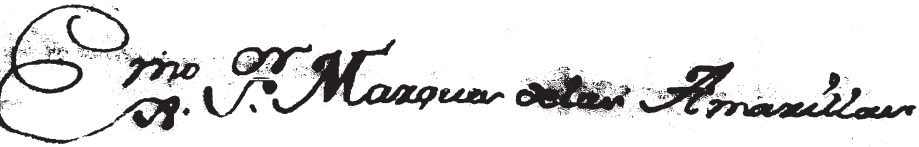

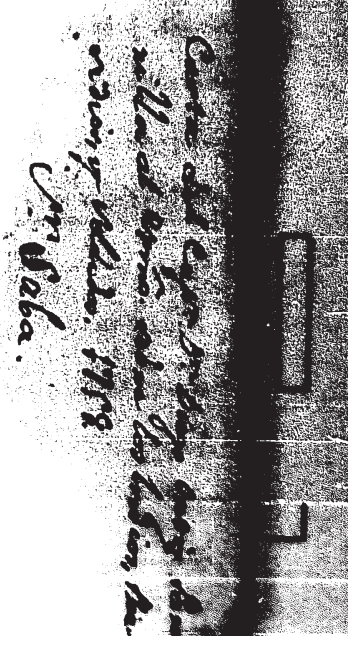




\section{[fol. 8r]}

por lo que se podra padecer mucho con-

tratiempo, en caso de ataque, cuando son los Yndios Enemigos tan astutos, y de tanto numero de Personas âsustadas; y en el campo podran experi-

mentar las Caballadas y Recuas de Bastimentos superdicion, sin que lo Remedie el cuidado, y la Guardia de Gente Presidial que âtodo se âplica, por que la muchedumbre de Barba-

ros lo benzera todo, pues logran espiritu, conducta, y el higual harmamento defuego al de nuestros soldados, que son infinitamente menos que los Enemigos.

II Nuestro Señor Guardê la importante Vida de Vuestra Excelencia los muchos años que deseo. San Luis de las Amarillas y Jullio 4 de 1758 \{rubrica\} Die go Ortiz Parrilla =

\{Archival reference\} $\mathrm{K} \mathrm{N} 8$

\{Archival reference\}Legado 8.

\{right margin vertical\} Carta del Capitan Don Diego Ortiz Par rilla al Excelentisimô sobre los Indios, Presidios, y soldados. 1758

San Saba. 


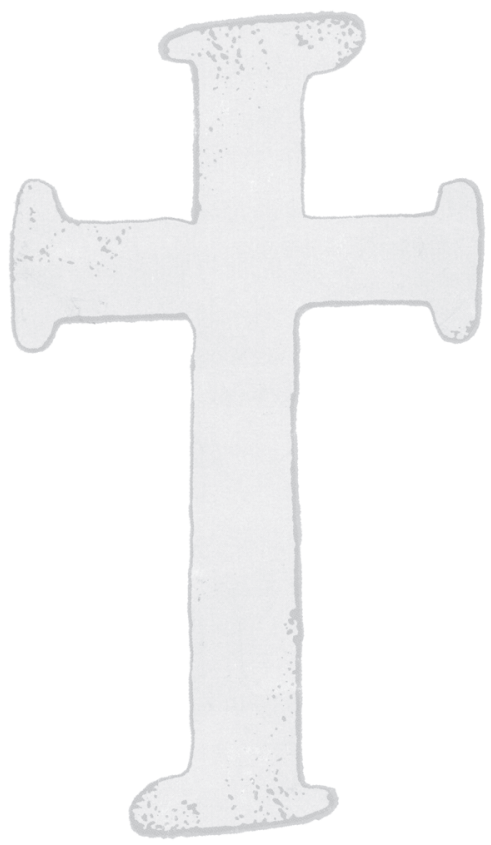




\section{ENGLISH TRANSLATIONS}




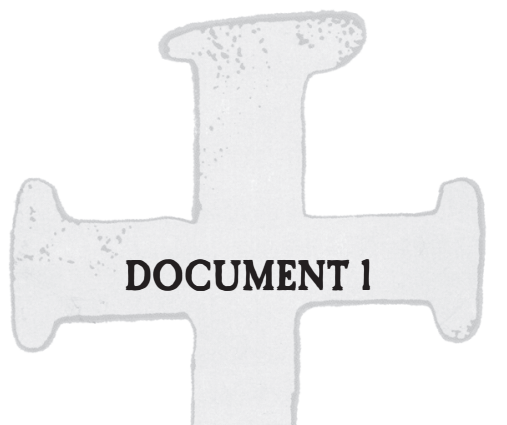

Subject: This document is part of the proceedings recorded by Colonel Parrilla, the military commander of the Presidio San Sabá, following the arrival of the Ypande Apache at the San Saba River and after the official Spanish survey of the lands chosen for the missions.

Synopsis: Colonel Parrilla summons Fr. Alonso de Terreros, Fr. Santiesteban, and Fr. Ximenez to survey the Apache campgrounds. He discusses the conversation he had with the Apache Captains Chiquito and Casaca Blanca, the layout of the campground, the Apache resources, and the difficulty of determining how many Apache were present. Apache Captains Chiquito and Casaca Blanca appear to agree to settle in a pueblo mission after returning from a buffalo hunt.

Paleography: As far as I can determine, this document has not been transcribed paleographically or translated before, although various people working for the Center for American History have transcribed some of this material.

Location: This document can be found at Material from Various Sources, Box $2 \mathrm{Q} 255$ v. 23, pp. 138 143, Center for American History, the University of Texas at Austin.

On the $25^{\text {th }}$ of said month and year [May 1757], having sent notice to the Reverend Missionary Fathers for the purposes that can be understood from the previous Auto, the Reverend Father President Fr. Alonso Giraldo de Terreros, Fr. José de Santisteban and Fr. Diego Ximenes arrived at this campground a little after three o'clock in the afternoon. They could not arrive before because it rained copiously since the previous night. Already present were the Captain of the Apaches, known as El Chiquito, with other Indians of the same [group], the Lieutenant Don Juan Galvan, four soldiers, and myself, the aforementioned Colonel. Without special protection, as if we were taking a regular walk, we rode our horses through the large number of tents and many tents, I mean, gentile people 
camped near us [military camp]. Having seen on all their faces the pleasure and satisfaction they feel, and the trust and good faith they place in our words, we returned to my tent, without having been able to count the people who make up such an immense group of gentiles. We could not even count the tents they inhabit because of their uneven and confusing distribution due to the hilly terrain where many of them are located. Despite the estimations made and the correspondent large number of horses and mules we could see, we were unable to obtain an account with the necessary formality and precision for a juridical report. Such an account would not have been difficult to obtain // but we did not think it was convenient [to do so] because of the knowledge of the mutable and suspicious temperament of the Indians, and because we can do so at the time when it is certain that they will formally settle with the aim to be reduced, as we wish. In light of this purpose we informed said Captains Chiquito and Casaca blanca as well as many of the more important Indians [principales] who also came to my tent, that they were to gather the following morning and join the Reverend Apostolic Fathers and me to survey the lands chosen. We also persuaded them to occupy those lands with their tents and formally settle in order that we could inform them of the benefits, relief, and comforts we have to offer. We spoke to them in a style proper to their rusticity until we managed to make the matter clear and explain the motives of [Christian] piety that lead us to promote this matter. We also entreated them to declare the clear wish and truthful intention of their presence as well as their union with us. In this regard, the aforementioned Captains and other Indians present // stated they would do as we proposed, and that they would send notice to those who were absent because their will and wish was to settle in mission-pueblos. However, they would never settle in totality at this time, because some were absent hunting buffalo. Many had left for the hunt on their way [to San Saba], and others were to go and pick them up in order that they be together because of the danger posed by the Comanche Indians, who are their capital enemies, but the women and children would settle under the conditions proposed. Regarding the work they had seen the Mission Indians perform they stated they would not do it for now, because they did not know how to do such work nor were they used to it. This objection found satisfaction [in the words] of the Reverend Father 
President and the other missionaries who offered to accommodate them [the Apache] and told them they would be treated as sons. I also told them about all the things with which the King favors and helps them. To this they did not retort and agreed to meet the following morning to survey the proposed lands and water sources. Captain// Chiquito informed that he had sent messages to invite many other Indians, besides those in the campground, in order that, like him, they would settle and reduce to pueblos. Hearing this news we tried to encourage him to send more messages and we followed that with a discussion of other lands and water sources which would be adaptable to this Nation and appropriate for their reduction, because this River can sustain only the two pueblos projected as the river does not carry water for more irrigation than the two water diversions already surveyed. The Captains and other Indians present gave us news of other springs, rivers, and lands but we decided not to proceed with another survey until it becomes necessary and urgent because of the arrival of other Indians who may not have enough land to settle or be able to subsist in this country. We will keep that [information] for when the time comes. I certify that this [statement] was signed by the Reverend Fathers with me and those who assist me. Diego Ortiz Parrilla = Fr. Alonso Giraldo de Terreros = Fr. José Santiesteban= Fr. $/ /$ Diego Ximenez = Attest: Bernardo de la Puente Campo Redondo = Attest: Juan Manuel de Bustamante. 


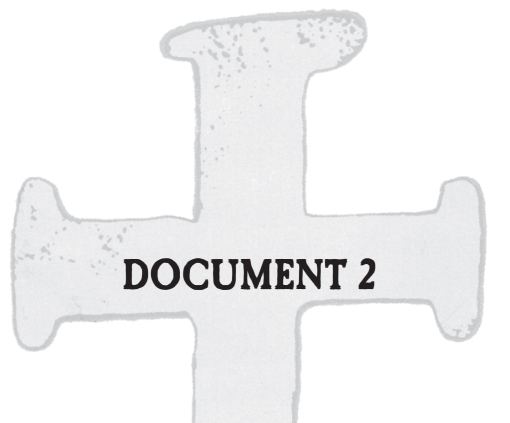

Subject: Colonel Parrilla summons Fr. Alonso de Terreros, Fr. Santiesteban, Fr. Ximenez, Captain Chiquito, and other Native Americans to survey the grounds chosen for the mission.

Synopsis: Colonel Parrilla together with Fr. Alonso de Terreros, Fr. Santiesteban, Fr. Ximenez, Captain Chiquito, and other Native Americans surveyed the mission lands. Captain Chiquito stated that he had sent messages to other Natives who had not come to San Sabá. The Captain stated that they found the lands suitable and would settle in them, though not everyone would do so immediately because they were to go on a buffalo hunt. Despite offers of food from the Spanish, the Apache did not change their plans. Colonel Parrilla and the Fathers decided to use all possible means of persuasion to convince the Apache to settle.

Paleography: As far as I can determine, this document has not been paleographically transcribed or translated before, although various people working for the Center for American History have transcribed some of this material.

Location: This document can be found at Material from Various Sources, Box 2Q255 v. 23, pp. 143145, Center for American History, the University of Texas at Austin.

On the $26^{\text {th }}$ of said month and year [May 1757] I, the aforementioned Colonel together with the Indian Captain Chiquito and other [persons] of his liking, proceeded to the campground of the Reverend Father Missionaries in continuation of these proceedings and considering the previous report about the survey and reconnaissance of the lands chosen and proposed for their mission pueblos. After the Father President and the other cited Missionaries joined us, we surveyed the mentioned land and impressed upon [the Apache] the favorable conditions of the land for settlement, encouraging them once again to promptly take possession [of the lands] and establish a formal 


\section{Spanish Colonial Documents Pertaining to Mission San Sabá}

settlement. To begin with said Captain Chiquito, spoke, as he always does, in the name of all [the others] because everyone happily accords him that [privilege] due to his character and the affection they all have for him. He said: that he would try to send all the warnings and news which he promised // yesterday he would forward to the Indians who did not come with them or with us to the event referred to in the previous report. As for his group, they would settle in the assigned land which he knew to be very good and appropriate for the purpose. First, however, those absent could not hear him, nor could they settle immediately as it was desired, and he repeated the news of the trip that many were to make to hunt buffalo and other animals of which they were in need. We again offered to supply them all with food that would eliminate the need to travel to the monte, and we assured them of all the conveniences and relief that I and the Reverend Fathers proposed. But every time we have tried to persuade them to quickly establish a formal settlement they have raised difficulties such as those expressed here as well as in previous reports, or other excuses of little import. Thus, I, the aforementioned Colonel, considered that without delay, it would be necessary to use the utmost persuasion at all times without losing any opportunity to achieve the settlement of these Indians. To this end I proposed to the Reverend Fathers to come tomorrow to my camp to entreat apostolically those Indians camped nearby who are indecisive and who have taken no action on what is offered to them and is so easy to execute. The presumed absence of some Indians is not sufficient motive to delay [settlement] because upon their return they can find their families in the place destined to be the mission without further work or difficulty as the lands [of the mission] are nearby and the trails to it opened. After the Reverend Fathers agreed to execute this proposal they signed this document with me and those who assist me, which I certify = Diego Ortiz Parrilla = Fr. Alonso Giraldo de Terreros $=$ Fr. José Santisteban $=$ Fr. Diego Ximenez = Attest: Bernardo de la Puente Campo Redondo // = Attest: Juan Manuel de Bustamante. 


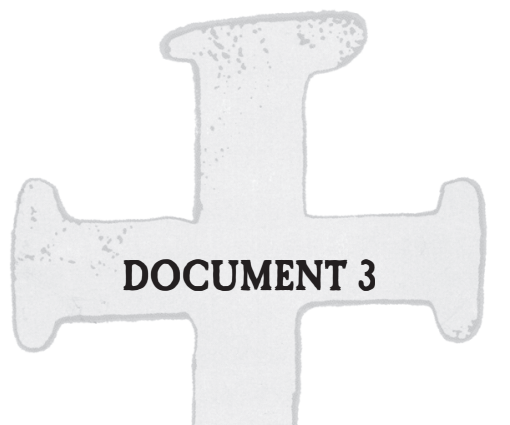

Subject: Colonel Parrilla summons Fr. Alonso Terreros, Fr. José Santiesteban, and Fr. Diego Ximenez as well as the Apache Captains Chiquito y Casaca Blanca to join him at his tent to once again persuade the Apache to settle in a mission.

Synopsis: Colonel Parrilla summons the missionaries and the Apache captains to his tent to once again persuade the Apache to settle in a mission. Parrilla and the friars reiterate the economic benefits and protection that will be provided to the Apache while emphasizing that the Apache will be vassals of the king and are to accept the Christian faith and the same terms and conditions as other mission Indians. The Apache declare their intention to settle in pueblo missions and state they will leave their women and children at the mission while they engage in an immediate campaign against the Comanche-for which they request the help of the Spanish, possibly in the form of weapons or troops. Because this document is missing one page, the exact nature of the Apache request is not clear. Fr. Ximenez remains at the military camp to exert his influence with the Apache and convince them to settle on the mission's lands.

Paleography: As far as I can determine, this document has not been paleographically transcribed or translated before, although various people working for the Center for American History have transcribed some of these materials.

Location: This document can be found at Material from Various Sources, Box 2Q255 v. 23, pp. 146149, Center for American History, the University of Texas at Austin.

On the $27^{\text {th }}$ of said month and year [May 1757], in compliance with what was agreed and decided in the previous report, at about half past seven in the morning the Reverend Father President Fr. Alonso Giraldo de Terreros, Fr. Josè de Santistevan, and Fr. Diego Ximenez, arrived and joined me, the aforementioned Coronel, in my tent. We agreed to summon to my tent the Captains of the Apache, known as el Chiquito y Casacablanca, as well as other principal people among the Apache to 


\section{Spanish Colonial Documents Pertaining to Mission San Sabá}

inform them of the objective of our presence and the easy means at their disposal not to delay their congregation in missions as we have proposed as well as to disabuse them of their ideas, objectives and thoughts, in order that well instructed and informed they can make their decisions and we can take the necessary measures and act according to their decisions. The Indians joined us at the first notice they received through the Indian Interpreter by the name of // Bartholome, and I, the aforementioned Coronel informed them of the motive for this summons. I reminded them that they had established friendly relations and peace with the Spaniards and with the Indians reduced in the old missions and of the many benefits and favors they received from everyone and the many, the many [repeated] times we attempted to persuade them to embrace the true Law we profess under the protection of the Catholic King, who cares for, watches over and favors all the Indians. This they have seen demonstrated in the Pueblos where they traded [interacted] and in the expressions [of friendship] that, on behalf of His Majesty, we have so often made to them. I further reminded them that they had given their word to settle and follow the law of the Christians like all other vassals of the King persuading and charging them with their commitments in the most efficacious manner. Lastly, I made them understand that their offer and pledges to reduce were made under the same conditions of those they knew and were applied to the Indians of the Missions of the River San Antonio and the Rio Grande of the Norte, // where they [the Apache] have been so many times, and with the supplies which they have seen us bring to this River of San Saba and which were furnished by the Great Capitan (as they call the Viceroy) in the name of His Majesty. These are the same provisions they are familiar with and which are used and consumed by the Indians who have accepted a Christian life. [Further], there is the weaponry of a large Presidio to protect, sustain and defend their lands from the enemy who might try to attack and cause them harm. All this was explained with the aim that they will resolve to settle the land destined to missions as we hope they will because they have so promised. The Reverend Fathers added other gentle reminders about the way they will be treated with true Christian love and tenderness and said Captains responded that they had decided their reduction and settlement of the lands and would leave their women and children 
because they wanted to leave immediately to go on a campaign against the Comanche Indians. They asked that we help them with that [the campaign] // missing folio // we proposed to dissuade them of so unjust a wish and in no way convenient to our military forces. Mistrusting their words, in the end we decided that the Father Lector Fr. Diego Ximenez should stay in the camp to pursue the advancement of these gentiles into the Christian fold that we have prepared for them within the short distance of a league and a half from where they are accompanied, I mean, camped. We informed the Father Preacher Fr. Benito Varela of all the steps and demarches performed since the arrival of the Indians to this River, and he agreed with everything and signed this report with the other Reverend Fathers and with those of my assistance to which I certify = Diego Ortiz Parrilla = Fr. Alonso Giràl de Terreros = Fr. Josè Santistevan $=$ Fr. Diego Ximenez $=$ Fr. Benito Varela = Attest: Bernardo de la Puente Campo Redondo = Attest: Juan Manuel de Bustamante.

\footnotetext{
${ }^{1}$ This page was not found in any of the volumes contained in Box 2Q255.
} 


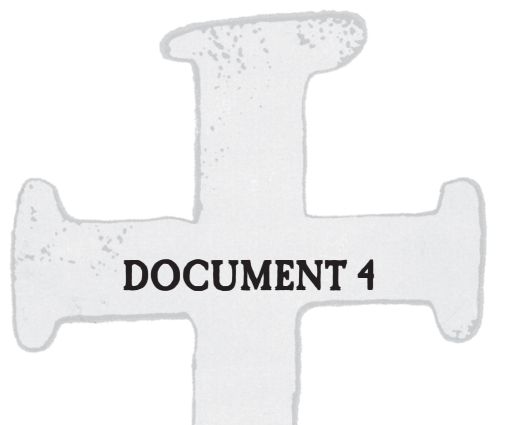

Subject: Colonel Parrilla continues with the proceedings related to the settlement of the Apache and the establishment of missions.

Synopsis: Captain Casaca Blanca and his people have decided not to settle in pueblos and reduce to mission, despite the offers made by the Fathers and the military. Captain Chiquito and 300 of his people seem to still desire to congregate in a mission. Captain Chiquito is camped with 32 tents, excluding his own, and has 300 people. Colonel Parrilla and his soldiers have managed to get an approximate account of the tents, number of people, and their horses. There are 311 tents on the campground near the presidio, and each tent houses between 10 and 12 people. ${ }^{1}$

Paleography: As far as I can determine, this document has not been transcribed paleographically or translated before, although various people working for the Center for American History have transcribed some of this material.

Location: This document can be found at Material from Various Sources, Box $2 \mathrm{Q} 255$ v. 23, pp. 161166, Center for American History, the University of Texas at Austin.

On said day, month and year [May 29th, 1757], I, the aforementioned Colonel, have learned that the Apache Captain named El Chiquito will be able to come to this campground, because the period of his duel ${ }^{2}$ has expired. The news I received from that Indian through the Reverend Father Fr. Diego Ximenez who has seen him // and has contacted him many times, lead us to believe that his will and that of his immediate kinfolk is different from that of the other Capitan and other Indians because, while these refuse to reduce and to settle in pueblos, Capitan Chiquito with three hundred people who make up the 32 field tents that adjoin his tent are offering to settle as we have proposed. I am also paying close attention to the said Captain Casaca blanca who, with another large number of 
Indians, is preparing to march out and leave these surroundings as they proposed yesterday amid the promises we reiterated to keep them in this Presidio, I mean, in this country, which is destined to Missions. We presented them with cattle [ganado mayor] for them to try it out because we had received in time part of the cattle that we asked for this purpose and therefore we could show them what we were offering. Surreptitiously, we also counted all the tents present in the camp which amount to three hundred and eleven. The number of horses // and mules, according to the corporals and other soldiers who are expert at making such observations, surpasses two thousand seven hundred of one and another kind. These observations were made in the field where the animals were being pastured and the estimate should be considered conservative because the total of the Indians, children and adults of both sexes, comprises the large number of three thousand. [One should consider that], there are three hundred people in the thirty two tents of Captain Chiquito and these tents are not bigger, they are not built differently or by a different method, nor do they [indicate] a different lifestyle, but the same practiced by all Apaches whereby three or four Indians and their families live in one tent, amounting to about ten to twelve people [per tent] as we can perceive from the account of Captain Chiquito and the thirty-two tents in his immediate campground. I obtained the same information from other sources and observations, and I should also state that the Indian warriors who can be seen are more than six hundred; some have French weapons and the rest have bows and arrows, and they are all // very dexterous in the use of both, and their spirit is strong and their will constant. Thus, they can make the war they are accustomed to with great satisfaction. Considering all this, I dispatched immediate orders to the corporals who guard our horses to move the horse herd to the immediate terrain and to a place appropriate to its safety; and to maintain caution, but reinforce the protection of this camp with more soldiers. We were already very vigilant exercising great care and guile in order that the Indians would not become wise to some distrust of their friendship and intentions, or that we are apprehensive and fearful of what could happen if their behavior changed because of their large number, their arrogance, their weapons and horses [and because they are] in their own land, while we are so distant from our settlements. This is even more so as we could not 
hide from them the small number of soldiers I have, since I have only thirty-four men, six who are sick and only one officer. Those who guard the horse herd and those who serve as escort in the campground of the Reverend Fathers amount to twenty. This means // that they are divided and I cannot benefit from their union because the guard of the horse herd is indispensable and the escort of the Missionary Fathers who occupy the land destined to the Mission where the Indians are to congregate is necessary. However, it is in this camp [the Presidio] that they now gather. Consequently, only the confidence in the planning for the attacks that might occur will make us remain in this country although I was unable to get the full number of soldiers for this Presidial Company because of the reasons already noted in other reports included in these Autos. [These soldiers] are not only necessary but also indispensable to fulfill the superior orders for the settlement in this river. Likewise, the distribution of the soldiers as expressed above is necessary, as is the order to call and summon the Indian Captain Chiquito and those of his following in order that they declare their wish and will [to settle]. We will follow with them the same reported steps and procedures as we did with Casaca blanca and the other Indians. Thus, I have provided for, ordered and testify in front of the witnesses assisting me for the motives expressed = Diego Ortiz Parrilla // = Attest: Bernardo de la Puente Campo Redondo = Attest: Juan Manuel de Bustamante.

\footnotetext{
1 This would result in a possible total of between 3,110 and 3,732 people. This total appears to exclude Captain Chiquito's 32 tents and his own, which would make 33 tents.

${ }^{2}$ Colonel Parrilla is referring to the presumed confrontation between Captains El Chiquito and Casaca Blanca.
} 
Subject: Colonel Parrilla continues with the proceedings related to the settlement of the Apache and the establishment of missions.

Synopsis: Colonel Parrilla and Fr. Diego Ximenez summon Captain Chiquito and other Apache of his following to ask El Chiquito to declare his intention to settle, but Captain Chiquito states again that he will join Casaca Blanca and the other Apache on the buffalo hunt and on the campaign against the Comanche, because he has been asked by the other Apache. Captain Chiquito promises to reduce to mission lands when he returns from the hunt and the campaign. Despite Parrilla's and Ximenez's efforts, Captain Chiquito does not change his mind.

Paleography: As far as I can determine, this document has not been paleographically transcribed or translated before, although various people working for the Center for American History have transcribed some of this material.

Location: This document can be found at Material from Various Sources, Box 2Q255 v. 23, pp. 166167, Center for American History, the University of Texas at Austin.

On the $30^{\text {th }}$ of said month, and year [May 1757] in the presence of said Captain Chiquito and other Apache Indians, said Reverend Father Lector Fr. Diego Ximenez and I, the aforementioned Colonel, informed them of the reason for this summons through Ygnacio the interpreter. I made the same reasonable arguments I used with Captain Casaca Blanca and which are detailed in the previous report and having informed him of everything, as we understood it, Captain Chiquito responded in the name of all the others. He stated, that "for now he could not keep his word to settle in the Mission with the three hundred people he promised, because Casaca Blanca and other Captains, as well as other Indians, had asked him with tears not to abandon them at the time when they had decided to 
make a campaign against the Comanche Indians, and to hunt buffalo in the land where buffalo can be found; and that he, moved by the care he has for all of them," // offered to assist them in these two endeavors. Once these endeavors are accomplished he will return to settle in Missions as he promised. He guaranteed this new promise with many expressions of reassurance and it was with sad demeanor that he said he had presented the news to his kinfolk and compatriots and that the two principal Captains reached a common decision. From what we observed about them in other circumstances they are Indians of renown among all the Apache. The Father Lector and I tried to tell them what was to their advantage and provided them with the information we had given to the others the previous day, but we could not persuade them to change their minds except for the future promises referred above. In light of this, and considering that we could not advance in our intent or will at the moment, we finished this report which was signed by the said Reverend Father Lector with myself and those of my assistance, and this I declare $=$ Diego Ortiz Parrilla $=$ Fr. Diego Ximenez $=$ Attest: Bernardo // de la Puente Campo Redondo = Attest: Juan Manuel de Bustamante. 


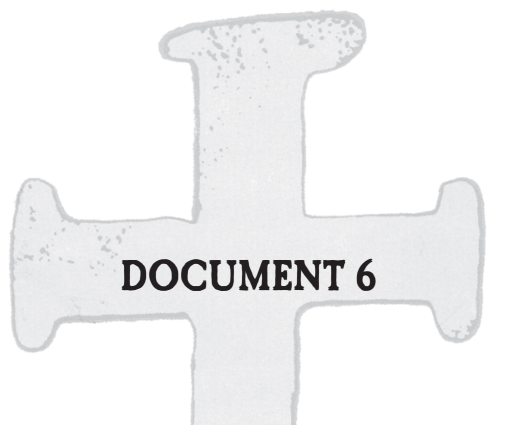

Subject: Colonel Parrilla continues with the proceedings related to the settlement of the Apache and the establishment of missions at San Sabá. He requests a formal certification from Fr. Ximenez.

Synopsis: Having realized that the Apache will not congregate in missions at this time, Colonel Parrilla asks Fr. Diego Ximenez to report on his contacts, results of his work, and understanding of the Apache behavior.

Paleography: As far as I can determine this document has not been paleographically transcribed or translated before, although various people working for the Center for American History have transcribed some of this material.

Location: This document can be found at Material from Various Sources, Box 2Q255 v. 23, p. 168, Center for American History, the University of Texs at Austin.

On said day, month and year [May 30, 1757] I, the aforementioned Colonel, considering what has been agreed with the Apache Indians and is recorded in the previous reports and the measures taken in this matter regarding their reducción to a mission pueblo after their arrival at this River, I state: that since one of those missions was destined to the Reverend Father Lector Fr. Diego Ximenez I declare for this purpose that I am aware of the efficacious measures and wise steps the Reverend Father has taken in this matter. [Further I declare] that he be praised, and I, the aforementioned Colonel requests, entreats, and charges that he certify all that he has done with the Apache Indians regarding their reducción to missions, the fruit that may have resulted from his Apostolic efforts and everything else the Reverend Father may find noteworthy about the matter, as well as the measures we have taken until now to fulfill the Superior precepts. And so I provided for, ordered and testify acting before the witnesses who assist me for the said reasons. = Diego Ortiz Parrilla = // Attest: Bernardo de la Punte Campo Redondo = Attest: Juan Manuel de Bustamante. 


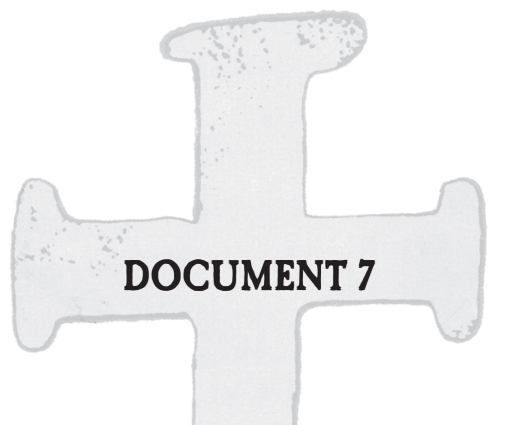

Subject: Fr. Diego Ximenez certifies to the steps taken to persuade the Apache to settle in a mission pueblo, to the fruits of his apostolic work, and to the knowledge he has acquired of Apache behavior.

Synopsis: In response to Colonel Parrilla's request and charge for specific information, Fr. Diego Ximenez comments on his contacts with El Chiquito and the reasons behind El Chiquito's refusal to settle at that time. Fr. Ximenez notes that his apostolic work consisted of baptizing two Apache, and he concludes by making a very perceptive summary of the possible scenarios that could explain the Apache behavior and what options are available to the Spanish regarding the conversion of the Apache.

Paleography: As far as I can determine, this document has not been paleographically transcribed or translated before, although various people working for the Center for American History have transcribed some of this material.

Location: This document can be found at Material from Various Sources, Box 2Q255 v. 23, pp. 169175, Center for American History, the University of Texas at Austin.

Fr. Diego Ximenez, of the Regular Observance of the Order of Saint Francis, Apostolic Preacher ex. secretary of Philosophy of the Province of Philippines and son of the College of Santa Cruz de Queretaro, destined to be the minister of one of the Missions of this River of San Sava, obeying the request of the Colonel and Captain Commander Don Diego Ortiz Parrilla, who // in the Auto of last May $30^{\text {th }}$ entreated, charged me, and on that same day informed me to certify to all that I have done with the Ypanes Apache Indians regarding their reducción to Missions, the fruit that was obtained from the Apostolic work, what I learned from dealing with the Indians in this matter, and about the methods used until now to accomplish what is demanded by the Superior precepts. I CERTIFY, and when necessary I swear in verbo sacerdotis about each of the above-mentioned points in the order 
expressed. Regarding the first point: I established contact with two Captains, and more frequently with the one who is called El Chiquito and some others, to ascertain their wishes to congregate in a mission and to persuade them to do so if it became necessary; said Captain (if we are to believe the interpreter) promised me to reduce to a mission in this River with all his people. He said that the number was about three hundred people of all ages and sexes, but that he would not be able to settle until he pleased the women who wanted to return to the Medina River to get corn, because they left [the Medina River] to this river after they had sown [corn]. I offered them a greater harvest in the mission // than they could obtain from their small planting to persuade them, by these and others means, to settle in the mission, but without success except for a variety of mixed news provided by this Captain. He told me that all his people, and a great many more he was waiting for, were to carry on a campaign against the Comanche, and that they were going also to the Colorado River to hunt buffalo, but once these two projects were finished they would settle here in a mission. I noted that all that was the artful doing of the enemy [the devil] who is fond of postponements and friendly to those who are damned in war. I offered them meat from our cattle to persuade them to go hunting at some opportune time after they had settled in the mission, but since I was unsuccessful I did not pursue the matter. Instead, I tried to persuade them to fulfill their word when they returned, because we, the Spanish, would be waiting for them. [I said this] thinking that at least while they were gone we would remain in this River waiting for superior orders. The other Captains and followers, who make up a very large number of people, though I was unable to determine how many, showed such subordination to El Chiquito that I thought they would do whatever he decided.

Among these Indians I found three apostates // of those I could not reach and who went to where they could live a Christian life; they told me about another five who did not want to come.

On the first day of my task I baptized sub condictione in articulo mortis [under condition and at the point of death] one male and one female of middle-age, brothers of the aforementioned Captain, and of those whom their kinfolk assured me wanted to become Christians, because I could not obtain that information from the patients as they were unconscious. The woman died right away and I did not give her a Christian burial because her kin refused it, and I did not insist much hoping to do so 


\section{Spanish Colonial Documents Pertaining to Mission San Sabá}

at a better time. I did not obtain any information about the man because his family left our area immediately. This is the fruit, though uncertain, of my labor.

I am not able to certify to what I have learned in this matter because of the lack of success in the measures we have taken. I will mention what I have observed without certifying to anything as completely certain and leaving the matter open to greater understanding. On one hand, the words of the Indians show the will to be missionized as does the fact that they came here for that purpose // [and were] sent by the Reverend Father President of the San Antonio Missions, Fr. Mariano Francisco de los Dolores. Furthermore, they came to this River where, in former times, they suffered so many insults from the Comanches, and [it is noteworthy] the easiness and pleasure with which they communicate with us and which we have experienced since they arrived. All this seems to indicate they want a mission. On the other hand, since they have visited us at the San Antonio Mission until now, they have not stopped asking for goods of their liking, and they have done this without the expectation that they will congregate in a pueblo, and I did not hear them make any declaration regarding their wish for reducción (though I did not lose any opportunity to persuade them to do so). Also, their determination that our weapons will help them against the Comanches before they settle in missions, together with not wanting to leave their women, children and invalids in our company [while they go on campaign], despite the fact that I strongly tried to convince them to do otherwise, seem to indicate little interest in reducción. If these Indians did not have the sagacity, astuteness and understanding they [demonstrate] in their negotiations and way of life we could attribute and excuse their attitudes as being rustic, but it seems that is not the case with them for which reason I leave this matter open. I do certify, however that // at present we cannot verify that the Apache are anxious for a Pueblo or a Mission. [This is] according to the Superior Government as is referred by the Auditor General in his report of July $27^{\text {th }}$ of 1756 on the subject of the Contract [Capitulacciones ${ }^{1}$ made with Don Pedro Romero de Terreros. These are the steps that have been taken with them [the Apache] up to now by the Colonel, who has spent liberally what he had no obligation to spend, and who has shown them all manner of hospitality, as has done the Reverend Father Fr. Alonso Giraldo 
de Terreros on behalf of the mentioned Don Pedro. These measures would be more than sufficient for the Apache to show their sincere wish particularly when again they were introduced to us [accepted] and they saw that we were already in their lands and came supplied with everything needed for their subsistence and for their defense against enemies.

Regarding the measures taken to accomplish the superior projects, I find them completely in accordance with the present purpose, which is the reducción of the Indians. In my humble understanding and according to what I have observed of their behavior, any other means would only upset and provoke them to acts of hostility and hatred of Christianity as it happened before. Also, other courses of action would lead them to deceive us while we were providing them with gifts and incurring excessive expenses borne by of the King our Lord, and by the aforementioned Don Pedro / / and by others. Thus, I am convinced that God has guided the executors of the superior projects with such prudence, wisdom and certitude that they have undertaken their respective tasks without deviation from due obedience, and without causing the disasters that issue from bad conduct. Also, we discovered the wishes of the Indians which, despite the contradiction of the events, still show brightly. Even though at present we cannot establish missions, if one reflects on the steps taken in these proceedings, which are known by everyone in this land, one cannot attribute its results to the bad management of those who undertook those steps, but to the indecision of the Indians, which results from their nature, the inconstancy of their promises, their large number, abundance of horses, and their arrogance. This I swear and expounded upon to comply with the charge referred above, in this River of San Saba on the $4^{\text {th }}$ of June of $1757=$ Fr. Diego Ximenez.

\footnotetext{
${ }^{1}$ Don Pedro's agreement with the Crown explicitly mentioned that the missions were for Native groups who inhabited lands north of Coahuila. This implies that the San Sabá missions could not be used for 'southern groups,' which annoyed Fr. Mariano de los Dolores, who wanted to send to San Sabá natives from San Antonio, and particularly those who were temporarily at the Guadalupe River. See Documents 8 and 9.
} 


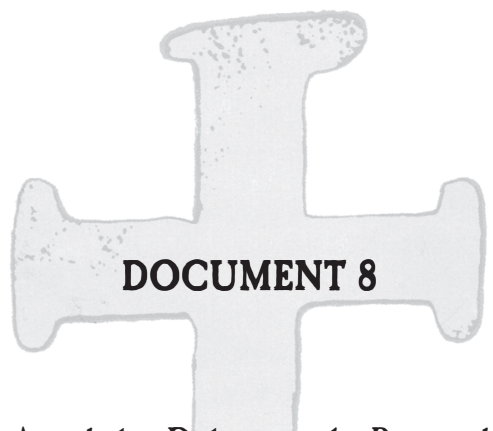

Subject: Letter from Fr. Maria Ano de los Dolores to the Reverend Fathers of the Rio Grande Missions.

Synopsis: As president of the Texas Missions, Fr. Maria Ano de los Dolores informs the missionaries on the Rio Grande of the events at San Saba after the arrival of a soldier who fled and survived the attack. At the time the letter was written, the people in $S_{a n}$ Antonio ${ }^{1}$ did not know who had survived the attack and what the situation was at the presidio. Rumors among the native population indicated that there would be other attacks, and the friars were making preparations for defense but were short of ammunitions. Fr. Maria Ano made the decision not to send native warriors to San Sabá, and he states that there were only five soldiers at the San Antonio presidio. ${ }^{2}$

Paleography: As far as I can determine, this letter has not been paleographically transcribed or translated, although various people working for the Center for American History have transcribed much of this material.

Location: This document can be found in the Archivo del Convento de Santa Cruz, Convento de San Francisco, Celaya Collection, Microfilm Roll 9:2719-2722 Old Spanish Missions Collection, Our Lady of the Lake University, San Antonio, Texas.

Reverend Fathers and Ministers of the Rio Grande Missions

Although I do not wish to renew the sorrow I feel or sadden Your Reverences with the report of fatal news, the responsibility of my job forces me to endure the pain and to ask the Divine Majesty to give us sufficient strength and resignation to withstand the pain we share.

On the $2^{\text {nd }}$ of March when the horse herd was between the Presidio of San Saba and the Mission, the Comanche, and other nations allied with them, took 62 horses. They were pursued by 19 
soldiers who returned without the horses but ignored the tracks they found.

On the $9^{\text {th }}$ said Indians attacked and wounded four Spaniards on the Pedernales River. These Spaniards were on the River de los Zanes [Chanas] working on some new and rich mines and having learned what happened at San Saba were preparing to join the mule train that was transporting supplies.

The Fathers were informed of these occurrences but the immediate measures that it is said the Colonel took in order that they would remove to the Presidio were not sufficient for the Fathers to abandon their post. And so it was that the attack occurred as described in the letter from said colonel, which is attached, and which I received on the $19^{\text {th }}$ of the current month.

The same day at 10 o'clock at night a soldier arrived who had fled the invaders at the same mission [San Saba], and who reported on what had happened. He stated that a very large number of Indians had arrived at the mission to take over // the post and that they picked up some goods, but the friars pretended not to notice to avoid confrontation, until it was decided to take the attacking Indians to the Presidio, though those same Indians took care to saddle the Reverend Father President's horse. The Father President mounted his horse and at the very moment of leaving they shot him dead, and in the same attack also died the Reverend Father Preacher Santistevan, a soldier, and Father Preacher Molina had his arm broken. Immediately the Indians disrobed the fathers, leaving the bodies on the ground, with their undergarments and sandals. The wounded Father [Fr. Molina] together with five soldiers, two Spaniards and a mule-train driver took refuge in the storeroom on the $16^{\text {th }}$ where they resisted all day. With some dead and some with less serious wounds as a result of the attack, that night the Father Fr. Miguel, Nicolas, the mule-driver, and a soldier decided to escape through a small hole they made. Nicolas escaped without being detected by the Indians (but we do not know what happened to him). The soldier also escaped with the Father (who was certainly already very fatigued) because the soldier was carrying him, but tragically the Indians detected them, shot at them and one bullet hit the soldier who fell unconscious. When the soldier came to, he did not see the Father, nor did he learn what happened to him; and it is to be surmised that if said Father did not perish, they took him captive. Here we think that because the bullet struck the 


\section{Spanish Colonial Documents Pertaining to Mission San Sabá}

soldier in the back, as seen by the wound, it probably traversed the body of the Father. That left the bullet without strength to do greater damage to the soldier and he did not see the Father because it was not yet daylight. He also states that the women and children were alive but barely, and that the Indians set the Church and everything else on fire, sacked everything and split the spoils between themselves. To me it seems that they will not leave anything // because they were killing even the cattle in the corral. All this the soldier reported and he swears by it as an eyewitness and he affirms it all happened as he declared. As of today, we have not heard again from the Presidio; we only had the two soldiers who brought the letter from the Colonel, and after the letter was read, the Lieutenant who had left his cargo in the fields, hurried back with 18 men, soldiers and mule-train drivers, and we do not know if they reached the Presidio [Las Amarillas].

The events at San Saba led to rumors among the Indians of these missions [San Antonio] that the enemy would invade Guadalupe and these pueblos [San Antonio]. For this reason I have ordered all the population of Guadalupe to be brought to this mission [Valero] until [Guadalupe] has sufficient protection or we see the end of this disturbance. The enemy is very numerous, and all missions are making the necessary arrangements to resist the enemy, if it comes, with the help of God.

For this reason, I decided not to send Indians to help, reasoning that to send few was to give the enemy more victims, ${ }^{3}$ and to send many meant we would be left without people for the defense and exposed to clear danger. This means that we have a maximum of 500 men of arms in all the missions; all together they are not competent to face the enemy in an open field, but they can be if shielded by their missions where the chief warriors have been killed but they have been replaced by gentiles and returnees [???].

The Presidio has only 5 soldiers and so the Captain [Thoribio Urrutia] cannot render help and I have only // persuaded him to send news to Los Adays, Bahia, Rio Grande, ${ }^{4}$ Coahuila, Santa Rosa and to the Kingdom in order that they send squadrons [to help]. This is to ensure that if San Saba is to be finished the same does not happen to the rest of this Missionary Province because this kind of war has never been seen. If San Saba is not to be closed, we may be able to punish such 
overwhelming menace with the people who may come, with the military forces that may remain, with these pueblos, and the alliance with the Apache and other nations.

I immediately notified the Apache nation of what happened, and though they promised me that a large number of them would render succor I am not convinced until I learn from San Saba the result of their promise.

This is the state we are in, and although I would like to inform the Reverend Father Guardian, the urgency of the matter does not leave me time. Thus, I beg your Reverences to find a way to forward to him the letter attached in order that he is notified and that both Colleges [Queretaro and San Fernando] take the measures deemed convenient.

I will appreciate if, with all diligence, Your Reverences request a carga ${ }^{5}$ of lead and as much gunpowder as you can, and send them without delay by Indians of those missions, as these missions are short of ammunition. And in the letter you will write to the College ask them to send these items to each mission.

It will please me that Your Reverences enjoy good health to which end I offer that which God has given me; in your sacrifices and prayers Your Reverences ask His Divine Majesty the remedy for so many evils that threaten us; and I will beg God to keep your lives for many years. San Antonio Mission, March 21, 1758.

\{left margin, vertical\}

The President of Your Reverences and your loving friend and servant

\{Left margin, vertical rubric\} fr. Maria Ano francisco de los Dolores

\footnotetext{
1 The information transmitted in this letter contradicts in some points the information provided by Fr. Mariano de los Dolores to Colonel Parrilla regarding the number of troops available in San Antonio and who actually decided not to send reinforcements to San Sabá. See Nathan and Simpson, Document 46, pp. 123126.

2 There is no mention of the nine soldiers Parrilla complained were retained in San Antonio despite the need for their presence at San Sabá.

${ }^{3}$ It could also mean "to increase the enemy," because cebar has both connotations.

${ }^{4}$ Text on the top left margin: "As soon as this storm is over we will take steps to recover the ashes of the Fathers and whatever belongings of Don Pedro Terreros may have survived."

${ }^{5}$ The value of a carga changed through time and according to region, but it was generally equivalent to 12 arrobas, or about 300 kilos (1 arroba $=25$ kilos).
} 


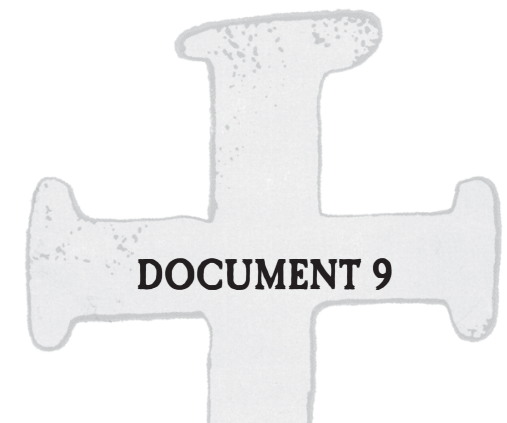

Subject: Letter from Colonel Parrilla to Fr. Mariano de los Dolores, Father President and Visitador of the Texas missions.

Synopsis: Colonel Parrilla describes and justifies his conduct and the disposition of his troops on the day of the attack on the San Sabá Mission. Parrilla complains bitterly about the citizens in San Antonio who gossiped about the events of that day and about Parrilla's failure to intervene to save the friars and the other people who perished. Parrilla wrote several letters to influential people who would decide on his future as a military commander in Texas, or those whose opinions could affect that decision; Fr. Mariano de los Dolores was one of the latter.

Paleography: As far as I can determine, this letter has not been paleographically transcribed or translated before, although various people working for the Center for American History have transcribed much of this correspondence.

Location: This document can be found in the Archivo del Convento de Santa Cruz, Convento de San Francisco, Celaya Collection, K N9 Legado 8, Microfilm Roll 9:ff. 2687-2704, Old Spanish Missions Collection, Our Lady of the Lake University, San Antonio, Texas.

Reverend Father Visitador Fr. Mariano de los Dolores

My dear sir, and much-esteemed friend. It was late [when] the Chaplain of this Presidio and Lt. Don Joseph Escaimuzquis [Ecay y Muzquiz] arrived at this Presidio. They delivered to me Your Reverence's letter, which I cherish and appreciate. With that letter they informed me of your resolve to travel to Mexico and the confidential news you relayed to him reiterated the kindness with which you favor me. I truly wish Your Reverence the best of luck in everything.

The mordacity of the people of that Province exceeds that of all the other places in the world 
and the truth [of that] will, in time, result in the deserved punishment.

It may be that the knowledge I have // of their situation and machinations will cause me to be the instrument of their punishment, though I do not seek it, nor is it my nature to occupy myself with such matters.

I live too far from those people to be concerned with what is said, for better or for worse as the saying goes, yet I have not succeeded in evading their calumnies, lies and gossip.

It is true that it pains me to see and learn how badly all the settlers of that River of San Antonio steer their projects and efforts to try to improve and ameliorate the conditions of such a sorrowful country. But despite my good inclination there is nothing else to be done but recognize and experience their errors, and wish to remain far from their neighborhood and contact, leaving them to enjoy the well deserved misfortunes, considering the satisfaction they experience in being miserable.

I also can assure Your Reverence // how sorry I am to have put so much care in informing the Capitania General of the most appropriate means for the improvement and welfare of that country. Alhough my advice may not be found in the decisions of superior officers, at least it shed light [on the matter] and [was] a way to introduce the measures the Province of Texas needed so badly for its relief and stability. This is what I now feel, as I recognize that those who would enjoy the wealth and benefits do not deserve them.

The list included is that of the soldiers who were in this company the day of the attack by the barbarous [Indians]. The soldier's destinations have already been provided to the Capitania, as I sent them a true report, a thing not done in these lands, in the same report I sent to the Viceroy.

Your Reverence knows well that this Presidio has one hundred soldiers // including the Capitan and other officers, and looking through the list, one will find that all were accounted for. This is easily confirmed because if one asks those listed where were they assigned and their duties, they would answer precisely that they were soldiers and that they were occupied with the assignments as I stated and informed my superior. This [explanation] will do away with the calumny, while those who voiced it will be credited with the punishment that surely I will request.

I did not call on the Indian aggressors, nor did I persuade them to attack the place or take 


\section{Spanish Colonial Documents Pertaining to Mission San Sabá}

the lives of those who perished; I did not know them nor did I get advance notice from any of them. On this issue I am also innocent from the rumors that spread among those country bumpkins and among others who should keep in mind that I did not assign the Reverend Father Fr. Alonso or his companions to the San Saba River or to the Apache missions. They were not // at the destroyed post to please me, or because of any decision inherent to my authority.

The measures I undertook in order that the Fathers would come to live near this presidio are known, and all the care I extended to the friars and their interests is notorious. It is known that I went out of my way to find means to make sure they were successful and in good standing in the commission that brought them to this place.

The Fathers acknowledged and were thankful for my efforts, my appropriate conduct and my steadfastness regarding the establishment [of the mission] in such desolate and dangerous country. The deceased Father President wrote a letter to the Viceroy a few days before his death, and in it he stated precisely that, and he asked the Viceroy to acquiesce to my return to Mexico for the purpose known to your Reverence, and which was completely dedicated to the glorious Statutes // and Rules of the Members of the Apostolic Colleges of Santa Cruz and San Fernando. Father President Fr. Alonso de Terreros perished with this satisfaction, but my detractors want to bury that too, showing their ignorance of the obligations of the deceased Fathers in this country as well as those that pertain to my job and my commission.

There were no missions, not even a mission as your Reverence knows; and the permanence in this country of the Fathers as well as mine was, and is, temporary according to the established rules and dependent on the orders we were awaiting. Considering all of this, I assigned a fourth of the men in this company to the protection and assistance of the missionary Fathers in the manner that follows.

I am referring to the hour and instance when I received news that there were unknown Indians in the house of the deceased Father President, because that is the [relevant] subject.

For the escort of the // eleven families and some women who were in the cattle ranch [Ganado mayor] I assigned seven men. Although under my name, this ranch was designated for the expected 
reducción of the infidels as well as for the subsistence of the friars and their family. Those men slept and arose in the ranch [always] armed considering the five-league distance between this presidio and the ranch. Indian tracks as well as some Indians who let themselves be seen nearby on different days persuaded me to take that precaution not long before the disaster. Beforehand only the servants were kept as such and [saw to the security of the ranch].

The four soldiers who were at the Guadalupe [River].

The nine who left the Presidio to reinforce the escort of the mission, and the five who were with the missionary fathers when they received the Indians. This totals // twenty-five men and consequently one fourth of this company.

It would seem that with this distribution and the care with which it was planned, together with the precaution that on the post [place] and at the side of the Fathers there were other men who could take up arms kept in reserve and use the ammunition and the artillery pieces which I also had [set] as prevention; the missionary Fathers were not unprotected nor were they without the corresponding precautions. The Father President and his family could have lived under the eaves of the Presidio, or even within its grounds as he was asked and entreated to do so many times. Inside the Presidio there was [enough] land for the necessary separation [between the friars] and the other residents. The unhappy events resulted and were unleashed by the refusal and opposition of the Father President [to move to the presidio]. For this reason one should feel sorry for the women and children and other people who suffer because they lost their husbands, fathers and kinfolk. Likewise, I should be // justly considered and paid for my losses and because I opened myself to censure and to the sharpness of the vengeful arrows shot by the ignorant folk who are normally more numerous in the world than those who reason.

For this reason, and many others, I am accredited with the good words and the good offices of the two Colleges, of their members, and of D. Pedro de Terreros and with [the fact] that they will always wish my participation in their projects because I have been such a good friend and companion to them. 


\section{Spanish Colonial Documents Pertaining to Mission San Sabá}

No doubt I could have used the power of my position to compel the Fathers to live somewhere else since I considered that convenient; but there is also no doubt that had I determined to do so I would have caused a contest which would certainly result // in a declaration of universal powers and capabilities of one group versus the other. That determination would cause differences of opinion and conflicts not fitting to a priest or to my desire for peace and understanding. For these reasons, I let the Fathers follow their wishes, and I did not oppose the [will] of the Father President to sow four or five fanegas of corn, as it can be seen today by the land he had cleared for that purpose, even before having Indians in the mission or being certain that he [they] would remain here. For this reason I am persuaded that sending Indians from the missions of the San Antonio River to this place would have been resisted, which was to happen under your Reverence's authority as I was given to understand by the Father President.

Considering all that has been said, and that a fourth of the military force of this Presidio was detached to // protect and assist the missionary Fathers; that the other three fourths were very busy performing their duties outside and inside of the Presidio; that the most prudent task was to protect so many women and children from the tyranny and barbarism of the gentiles because these are people just like those who were in the destroyed mission; and considering further that the [mission] was already destroyed by the time I learned of the arrival of the Indians; what else could I have done to deserve the applause of those who are prudent and knowledgeable? [All that could be done] was to preserve what the treacherous intent of the barbarians did not destroy. This they failed to do not for lack of vigor but because their intent was understood in time and for many other reasons which deserve better explanations than those I owe to the settlers of the San Antonio River, who, if they were thoughtful, would have understood that // fate thwarts the best laid plans, but cannot take from us the glory of having followed the rules of prudence. It should suffice that a man does not fail to do anything to achieve his aims. In fact, the good or bad outcomes should neither increase nor decrease the praise.

Such is the reasoning of those who know more than I do, and I strive always to imitate them, 
and disregard the opposing opinions.

In this manner we dealt here with the attack, not with temerity or ignorance, which appears to be the evaluation of my detractors from that place [San Antonio], who have proposed wishful methods and tactics while they remained in safety and tranquility. I believe that if they were in charge and exhausted they would reflect otherwise, or they would not reflect at all which would be more // likely.

The people in those settlements [San Antonio] thought that the enemies were located seventy leagues away, or at least should have thought that, had they thought about it at all. With such a great distance, and even though they did not feel safe, their sole precaution and prevention was to remain within their precincts and houses, and kept there [San Antonio Presidio] nine soldiers which belonged to the land invaded, the land that suffered a surprise attack, and where people got killed by enemies. Even so, they have the audacity to pass judgment [laws] on those who are absent, without the presence of the proper authorities of the country and with no knowledge of the real events.

This is what is going on in those settlements [San Antonio], and I tell this to Your Reverence because I am strengthened by the understanding and Christian piety with which you listen to the news and for the relief of confiding in a friend // as true as your Reverence has been to me.

Those who came out of the mission alive were freed because of my conduct and not for other reasons that are suggested. I should add that the measures I took were in accordance with the situation, with the events, with the [military] forces I had, and for the protection of the settlement.

These were not decisions such as one sees in ignorant countries where easy, thoughtless decisions are made; my conduct was the most prudent and most useful.

At nightfall on the day the Indians arrived at the mission and occupied all the surrounding area I sent a sergeant and fourteen men with two Indians from those settlements [San Antonio] with orders to reconnoiter the land and attack the barbarians if that was possible, or to inform me if we could attack // with more people. [They had orders] to enter and survey the mission, which since nine in the morning we thought had been destroyed and the residents killed due to the deceit used by the 


\section{Spanish Colonial Documents Pertaining to Mission San Sabá}

gentiles to enter [the mission], according to the news relayed by those who saw it all burned. [These soldiers were also to reconnoiter and survey] the area occupied by the enemies or that they might have used to retreat, if they were no longer there, in which case they had other appropriate orders.

This squadron was detected by the barbarous Indians as soon as they arrived, as confirmed by all soldiers because the Indians immediately began shouting to each other and [the Indians] deployed themselves with such aim and coordination that they could have taken the lives of the squadron soldiers and many more according to the report of the sergeant and his companions. Thus, the soldiers returned to the Presidio leaving the Indians on alert and on the lookout over the roads // and over all the mission buildings that were burning so brightly they lit up the grounds.

Because of the noise they had made and trusting the fire, the gentile Indians carefully sought refuge confident they would defeat those who might attack them; and for that reason they chose an advantageous spot and left somewhat unprotected the small dwelling where the survivors were hiding, making it possible for them to escape. In this manner it was possible to free them, keep the Indian enemies occupied, and not sacrifice to the furious attack the few people who will maintain and protect this settlement and its properties.

The number of enemy Indians was exceedingly large; and one must believe this despite the information from the Orcoquiza Indians and that from the citizens of the San Antonio River. We must trust what I say and what is being said by a friar, as // attentive to details as is Father Fr. Miguel Molina, who saw them, was wounded by them, and even talked to them, as well as to what other men say who shared the friar's experience.

Even if the twenty-five soldiers mentioned as auxiliary force to the missionaries had been in the mission they would have suffered the misfortune of the surprise [attack] because of the astute way they [Indians] used to enter [the mission]. [In that case] we would have experienced greater losses, and even complete ruin, which is credible given the sound reasons that emerged, which I will expound upon if it happens that I am charged to do so because of the town meetings those dissatisfied may call. 
They will make accusations and I will know very well how to respond to them, and they will not be able to contradict the logic and strength of the [accusations] I will make, nor will they be able // to present to the superior officials and to Don Pedro de Terreros that which I will put before their eyes if they provoke me to do so, or if they bother me in any way. But I will not do it before, as the entire world will know, because such is my temperament and my upbringing.

God help us with citizens such as those from the Presidio and Villa de San Fernando in the San Antonio River, and how much have they caused me to go on about this subject, keeping your Reverence's attention and taxing your kindness. I beg your indulgence and whatever might please you to order me, as I ask God to guard the life of Your Reverence for many years to come. San Luis May $22^{\text {nd }}, 1758$.

I kiss Your Reverence's hands, your very good friend, and servant \{Rubrica\} Diego Ortiz Parrilla 


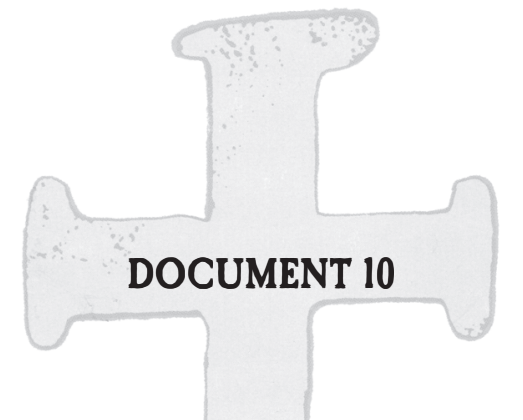

Subject: Letter from Colonel Parrilla to the Viceroy Marqués de las Amarillas. Parrilla reports on the arrival of the Lipan Apache at Presidio San Sabá. Parrilla uses the information provided by the Apache to discuss problems with the military in Texas, the citizenry, the Apache, the debacle at San Sabá, the Native American groups that attacked San Sabá, and the campaign to be waged against them.

Synopsis: Captain Chiquito (Tacu)and other Apache arrive at Presidio San Sabá after having been attacked by the Yujuane. The Apache provide information about the location of the Comanche and their strength. Colonel Parrilla uses the threat represented by the Comanche to stress the correctness of his decisions during the attack on the mission, the poor conditions of the Texas presidios and their troops compared to Presidio San Sabá, the trade with the French, the involvement of the Tejas, and the ineptness of the San Antonio citizenry. Parrilla's evidence and arguments seem to have two objectives: to salvage his reputation and to gain permission to command the punitive expedition to be undertaken against the Norteños.

Paleography: As far as I can determine, this letter has not been paleographically transcribed or translated before, although various people working for the Center for American History have transcribed some of this material.

Location: This document can be found in the Archivo del Convento de Santa Cruz, Convento de San Francisco, Celaya Collection, Microfilm Roll 9:ff. 2668-2683, Old Spanish Missions Collection, Our Lady of the Lake University, San Antonio, Texas.

\section{Dear Sir}

Among the Apache, which we know by the name Ypande, the Captain with more authority and most esteemed, is Tacu, or El Chiquito, as he is normally called. Your Excellency has already some information about him from previous judicial documents and Consultas. He arrived at 11:00 yesterday without a horse and fleeing from his Indian enemies who attacked him near the Rio 
Florido, more or less 20 leagues from this Presidio.

His jacket is pierced through in several places by the arrows, and one of the five [Indians] who is accompanying him showed me // the wound made by a bullet which cut through part of his face.

I received them with the usual demonstrations of friendship and hospitality and they reported that, three days ago, while on a hill in said land [Concho River area] where they were hunting buffalo, they suffered a surprise attack by a large group of Indians of the Yujuany nation, [a nation] that was also present at the treacherous and barbarous attack on the post of the religious missionaries in this river.

This Apache Captain and some of his companions had no choice but to flee, which they managed in the disorder caused by the attack, but they left tents, weapons, mules and horses in the possession of their enemies who sought them with greater vigor.

These Apache warned us that some of the traitors were dressed with spoils from the destroyed post of the Fathers and [the Apache] described them so well that there is no doubt that // these were accomplices in the premeditated attack of San Saba as well as in this [premeditated] attack.

They also tell of some loss of people between those who died or were imprisoned, and they do not think the enemy suffered any losses, despite the defense they managed to mount.

Another twelve, who also fled, followed this Captain and his five companions, and I received them with equal demonstrations [of friendship and hospitality].

They took different paths and arrived separately and at different times, but all agreed that the enemies are moving toward this presidio well armed, and with good and spare mounts [horses]. Three of the last group of fugitives witnessed, two nights ago, the movement of the enemies and the state of their forces, and according to what they said the [enemy] forces exceed theirs [the Apache] and ours because the Barbarians are more than two thousand.

They tell us, because of the information and knowledge they have of the [Indian] men, that the men normally built // a fire or a hearth to cook the meat they eat and the number [of fires] they saw results in the total number they have mentioned. 


\section{Spanish Colonial Documents Pertaining to Mission San Sabá}

This Apache Captain is very humiliated by all the persecution they have suffered and today he thinks only of revenge and ways to achieve it, and in that frame of mind he cannot think about reducción, [or about] settling and joining the missions as we desire. But he assures us of the faithfulness of his friendship and good relations, which are needed for the proposals [we made] and the continuance of our objective.

These Apache attributed the majority of the enemy to the gentile Comanche because they counted thirty-five fires [belonging] to the Comanche and in each one there were twenty-four men which, among them, is about the required number for the camps or divisions [separations] they establish during their marches when they are going to war or travel to an unknown country. The Apache state that the rest of the enemies were from the other nations allied [with the Comanche].

This Apache Captain // and the others have remained here for twenty days, coming from the Provinces of Texas and Coahuila, where this Apache Captain gathered many Indians of his following for the discussions [tlatoles] they have and visits they exchange. It seems they caused some worry in that land, and since this Indian is the one who reported it and of whom you had information, your Excellency will understand his character, and with your unique knowledge of the Indian land you will see what pertains to the matter.

The easiness with which they communicate and the manner in which they treat their belongings, particularly when they ignore them, is an engrained custom and so common to their people that I doubt they will ever be free from the influence of those who live farther away and who desire nothing else but to be restless and make trouble.

Even though I am far away from knowledgeable settlers and I write long and detailed letters to satisfy my temperament and to keep my mind from dwelling on bad thoughts as well as on news // that relate to my duties and commission, I have understood the variety of official reports made about the last events that took place on this river, regarding the type, condition, and nature of the gentile Indians, the damage and losses they caused, their weapons and their numbers. I also understood what we did and the precautions we took in this presidio at the time of the attack of the 
gentiles; and about other things, such as those referred, that are ignored by those who are dedicated to criticism and censure. I did not fail to do everything to achieve the desired goal of the reducción of the gentiles and the protection of the land chosen. I hope that the fair justification made by Your Excellency and by the ministers of your Capitania General will, in the end, declare a calumny and a lie all that has been written and shouted and which is not in agreement with the news I provided you, or with any news I might still give about any subject, because I always report the true facts which should result in legality, purity and // respect.

Since I came to this country, the law, prudence, and what is feasible have dictated all my actions and management, because what is impracticable is impossible to achieve. For this reason, it seems that unfortunate events should not count as demerits and disrespect because only fate can unravel the best laid plans when nothing has been omitted and fault cannot be found in anything that has been done up to now.

Had I behaved otherwise I would not have remained here, with the many advantages besides those offered by this country and its surroundings. With the happiness that all those who are pruent can present see, I could satisfy the calumnies in case they follow bad advice stand in the way and do not respect Your Excellency's superior position.

The projects and reports that contradict those I have made to Your Excellency about this land are the main subject, and they concern the invasion, the hostilities and the fact that here one looks the enemy in the eye. These conditions require a closer examination, as indicated in your Justification, than has been done by those who // planned those projects, as you will understand by the following news. The land we intend to occupy in this part of the North is not pacified as we would need, nor does it permit changes or control of the movements of the barbarians or to extend our reach to punish them as they deserve. In my letter of April the 8, I suggested to Your Excellency what I think is most convenient about these matters, and at the same time begged Your Excellency to give me the command and organization of this campaign because of the experience I have to conduct it appropriately, but also because it was the land of my commission which was treacherously 


\section{Spanish Colonial Documents Pertaining to Mission San Sabá}

attacked by the marauding Indians, all of which recommends me to Your Excellency's Justification.

Many Indians were allied for the tyrannical attack, and the Tejas Indians, with their presumed loyalty, did not excuse themselves from the plot. As much as they [the Tejas] try to hide their treason and infidelity, time will make it obvious. For your complete information I too will rely on time and on the procedures I will use in the field exercising the greatest effort until I manage to punish // the barbarians, and acquire the exact knowledge of the routes the Infidels use for the commerce and traffic with the French nation, as the Governor of the Province of Texas informed your Excellency recently. According to his warning and the news he passed on to me in great detail, some of my mules stolen by the Indians were in the French Presidio of Nachitos and in other French towns, as were some horses and objects the Infidels took from the place they treacherously attacked.

I did not say much in my last cited letter about the miserable conditions of the soldiers in these countries, though I could have used reams of paper to talk about them, but I expect those conditions cannot be hidden from your consideration given your keen understanding. Not to worry Your Excellency I omitted then what I will tell you now.

This Presidial Company is again formed, because although many served in the Presidios of San Xabier and of San Antonio, how could they be properly trained and disciplined under the conditions you know quite well, and which resulted from the actions of Don Phelipe de Rabago, former Captain of San Xavier, and from those of Don Thoribio de Urrutia who, while holding the job of Captain of San Antonio, suspended the Governor of Texas with denigrating and insufficient charges as I was informed by Don Jacinto de Barrios, when he asked me to send an interim officer to that Presidio [Los Adays]. Instead of learning their duties as soldiers they [Rabago and Urrutia] spent their time making mistakes and vilifying others, habits they added to their poor upbringing and to their cruel nature.

If reports on the condition of the soldiers, on the country, and on the Indians as well as the consequent thoughts bring me to this discourse, the comments about those who plan projects and scrap them would cover much more paper and cause perplexity in your Capitania General and to its 
decisions. This is because the variety of consultations and reports, the different colors used to paint the events will // raise some questions, but it is our good fortune to have Your Excellency's notable knowledge on the objectives of each person [or report], because, in this manner, everything is handled quickly and with all the equanimity and justice we experience, despite the problems mentioned.

In the past year and a half these soldiers have served under my immediate command. They have been occupied with many tasks, with marches, and with the rules they must observe. Despite that they have also received timely instruction and teachings corresponding to their job, as it is recognized and can be seen in their performance. But the short period of instruction and certainly their bad tendencies and upbringing do not let them understand the Honor [?], nor does it encourage them to strive to do better; as a result their performance does not do credit to the intelligence and conduct of their leaders. For this reason, much is the work we endure in these far away places and the continuous battle we wage while we do not fight the enemy although, at times, it would be more pleasurable to do battle and match // the greatest efforts of the Barbarians than having to manage and command so careless and timorous troops as ours.

By these statements I hope to convey that the Presidio de San Luis de las Amarillas can serve today as an exception to what I have said because it is being run well. Also, I can always state that all the [poor] conditions exist in all the other Presidios of these Kingdoms, but as the distance and difference in enemies make clear, my present concern is with this area because the soldiers are not trained as we need them to be, and the enemies have the advantages that the soldiers lack in the other Presidios. Still, my Dear Sir, that certainly will not dampen my spirit nor has any soldier left this post after the treacherous attack of the Barbarous [Indians], despite the consternation that one can see and which you must have surmised from the petition they made to be transferred elsewhere and that I attached to the Autos (Legal Process) prepared on the matter.

To convince them to remain here in the field // Your Excellency will certainly understand how much I had to apply myself and how artful I had to be because these are the same soldiers [who were present during the attack]. Furthermore, the country is so exposed and dangerous, as I am sure 


\section{Spanish Colonial Documents Pertaining to Mission San Sabá}

you understand from the news these Indians brought, from what has occurred before, from the frequent conflicts with the enemy Indians, and from the evidence of tracks of others who are all around us.

I consider the news I am providing Your Excellency about the state of the country and its enemies to be very important, and so I want to bring them to your attention without delay as it is my wish not to cause embarrassment to your Capitania General which I always consider to be thoroughly engaged in these matters.

I have written this correct assessment several times, [but did not forward it]. Others would have sent it considering the reasons and what has happened here, as I surmise because I hear that some people have sent [news]. Those people are far away from this land and do not know the truth and the circumstances of what happened, but they went ahead and sent news and advice// which I also consider different from mine and which I have conveyed at appropriate times.

I am including a letter I received a few days ago from the Reverend Father Visitador de Missiones Fr. Mariano de los Dolores because his news lends credit to those given by these Apache. I understand that the Father has gone to the Court in Mexico, but [I include this letter] because of his character, distinction, intelligence and the zeal with which he has performed his Apostolic work for many years in this country, and because your Excellency might have listened to him for the Justification [you wrote]. I do not apologize for forwarding this letter, as I do not need to find excuses to send any testimony that might accompany this news, which will be certified in time, if necessary. Such is the case of the Father Minister Fr. Francisco Aparicio, who is in this Presidio, and of the Father Chaplain of the Presidio and all others who live here because they are all aware that the information these Indians gave is the same that I report and they know everything I have expressed.

The situation of the country demands // [another commander?] for the Presidio [San Anonio?] and the proud Infidels need to be contained and more attention paid to the problem than it has received, as one can understand by the news I report and what I communicated before to Your Excellency in my cited letter of April $8^{\text {th }}$. I am sending this [information] convinced of my duty and 
with the desire that you remain well informed of all that is essential and appropriate, and I ask Your Excellency for your consideration and for the speedy deliberation that the matter requires.

The land I occupy with the people I command has no more protection or fortification than the one that was described in my previous letters, and is similar to that [protection and fortifications] which the missionaries had in their dwellings. Although the Presidio has been constructed according to some of the art and rules of defense it is made of materials easily ruined, because we have had neither the time nor has there been a decision about the precise fate [of this place] or the reducción. [For these reasons] there has been no assistance, and we have been unable to spend funds from the Real Hacienda or from those settled in the country; for the former I lack the authorization, and for the latter there is lack of capacity. Thus, there may be serious problems in the case of attack, particularly because the enemy is so astute and so many people are too scared. In the field the horse herds and the supply trains may be lost and the Presidial Guard, which has to do everything, will not be able to help. The large number of Barbarians will win it all because they have spirit, proper conduct, and firearms equal to those of our soldiers who are infinitely less than the enemies.

May our Lord Keep the important life of Your Excellency for many years is all I wish. San Luis de las Amarillas, July 4, 1758 \{rubric\}Diego Ortiz Parrilla. 


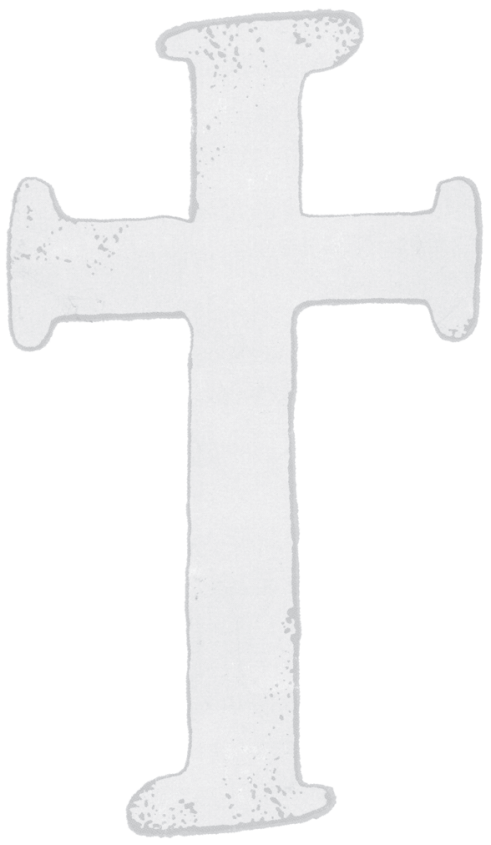

